\title{
RESIDENTIAL ENERGY MANAGEMENT SYSTEMS WITH RENEWABLES AND BATTERY ENERGY STORAGE
}

\author{
By \\ Aidan Brookson \\ B.E.Sc., Green Process Engineering \\ Western University, London, Ontario, 2015
}

\begin{abstract}
A thesis
presented to Ryerson University

in partial fulfillment of the

requirements for the degree of
\end{abstract}

Master of Applied Science

In the Program of

Mechanical Engineering

Toronto, Ontario, Canada, 2017

(C) Aidan Brookson, 2017 


\section{Author's Declaration}

I hereby declare that I am the sole author of this thesis. This is a true copy of the thesis, including any required final revisions, as accepted by my examiners.

I authorize Ryerson University to lend this thesis to other institutions or individuals for the purpose of scholarly research.

I further authorize Ryerson University to reproduce this thesis by photocopying or by other means, in total or in part, at the request of other institutions or individuals for the purpose of scholarly research.

I understand that my thesis may be made electronically available to the public. 


\title{
RESIDENTIAL ENERGY MANAGEMENT SYSTEMS WITH RENEWABLES AND BATTERY ENERGY STORAGE
}

\author{
Aidan Brookson \\ Master of Applied Science, Mechanical Engineering, Ryerson University, 2017
}

\begin{abstract}
With increasing concern towards the environmental impact of energy production, distribution, and consumption in the modern world, the overall energy landscape is changing. This Master's Thesis investigates methods of addressing these inevitable transformations through the incorporation of renewable energy and energy storage on the residential-scale using energy management systems (EMSs). A simulated residential house model was developed in order to compare a variety of different energy management techniques on the same basis. The simulated EMS investigation has covered: deterministic EMSs, those in their most basic forms; adaptive EMSs, utilizing machine learning and predictive control algorithms; and, a transactional EMS. The deterministic EMSs produced the least annual cost savings, but are the simplest to implement. Adaptive EMSs have shown the highest estimated cost savings, with increased controller complexity as a trade-off. The transactive EMS has shown intermediate cost savings, with additional potential benefits such as demand response and community integration capabilities. Experimental work has been conducted verifying critical claims of the systems, focusing on battery output control and inter-agent controller communication. The most interesting areas warranting future research involve implementing predictive control experimentally - and on a wider scale - and investigating transactive control on the community level.
\end{abstract}




\section{Acknowledgements}

This work was made possible through the financial support of various agents. I would like to thank the Mitacs Accelerate Program and the Toronto and Region Conservation Authority (TRCA) for providing the financial support to conduct the transactional energy-related work in this thesis. I would also like to thank Mark Riley from SUMARAN Inc. for providing financial support and acting as the driving force for the experimental air source heat pump/solar photovoltaic/battery load shifting control portion of this work. Additional funding was provided by the Centre for Urban Energy, Hydro One, and NSERC, which has aided in developing components of this work involving battery bank sizing and small-scale wind turbine incorporation. I am also grateful for the financial support of the Ontario Graduate Scholarship.

None of this work would have been possible without the support of many individuals. First and foremost, I am whole-heartedly grateful to my supervisor, Dr. Alan S. Fung, for his academic guidance and financial support over the last two years. I would also like to thank Dr. Kaamran Raahemifar for his academic encouragement - particularly during the early months of my time at Ryerson.

I am very fortunate to have been in good company throughout this journey - Navid Ekrami, Raghad Kamel, Rakesh Kumar, Afarin Amirirad, Altamash Baig, and Danilo Yu are all integral components of this research team, and their insight has provided great aid throughout the development of my own work, as well as that of many others. I would particularly like to thank Waleed Alzahrani for his aid and expertise regarding the thermal performance of buildings. I would also like to give special thanks to Kajen Ethirveerasingham, Edward Vuong, and Brandon Wilbur. Throughout our time at Ryerson, the friendships we have built through the countless conversations about our shared ideals of sustainability have been one of the most powerful tools in aiding in the progression of my work.

The Sustainable Technologies Evaluation Program (STEP) team's support has been one of the most integral components of not only this work, but my entire professional career. Many thanks go to Tim Van Seters and Dahai Zhang for providing me with my initial opportunity with the STEP team over five years ago. I would like to thank Amanda Yip for her aid in the coordination of the 
practical components of this thesis, David Nixon for providing invaluable technical wisdom, Svend De Bruyn for his aid with the battery bank and electrical systems, and Ricardo Brown for his tireless aid in remedying mechanical system issues. I owe a great deal of gratitude to Shivam Saxena, his software expertise has made some of the more complicated components of this thesis a breeze to implement.

I would like to highlight the support I've received over the years from Erik Janssen, Gil Amdurski, and Leigh St. Hilaire. They have provided me with indispensable knowledge and insight my years with the STEP team, and have helped create a constructive, highly enjoyable work environment. I would not be where I am today without their support throughout my professional and academic endeavours

I would like to acknowledge just how fortunate I am to have been born into a family that has been extraordinarily encouraging and supportive throughout my entire professional, academic, and personal lives. It is with their encouragement that I have pursued a life dedicated to sustainability. Finally, I am outstandingly grateful for the love and support of Lauren throughout this journey. I am incredibly lucky to be sharing this life with the endless support of my partner and best friend. 


\section{Table of Contents}

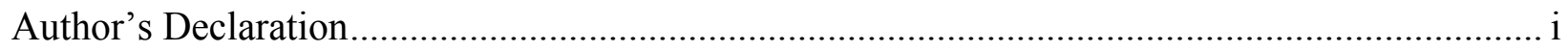

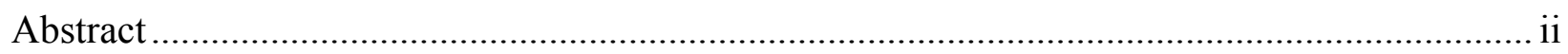

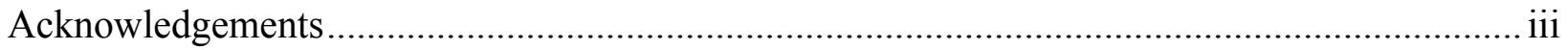

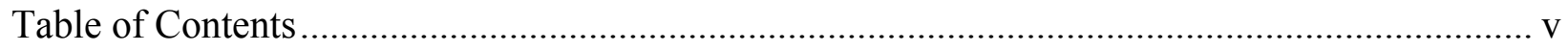

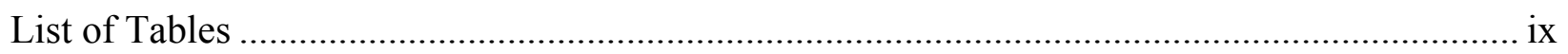

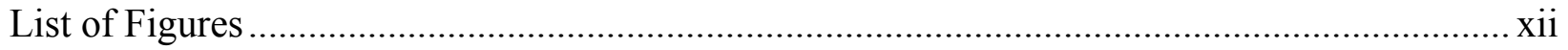

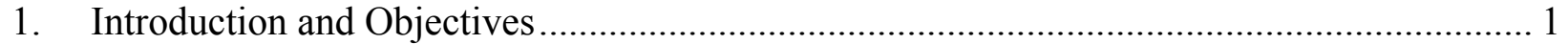

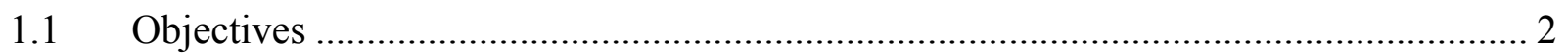

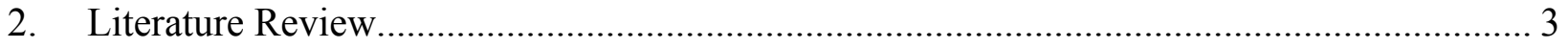

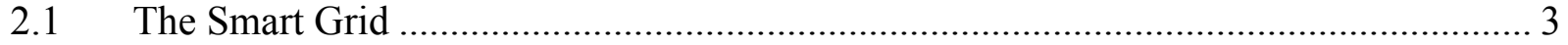

2.2 Net-Zero Energy Buildings...................................................................... 4

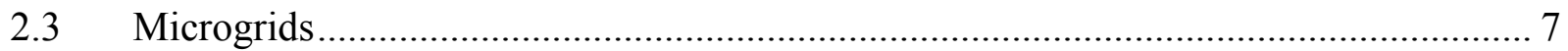

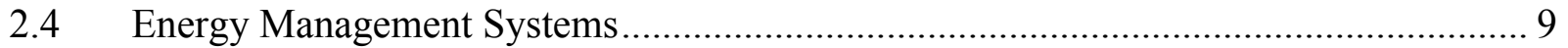

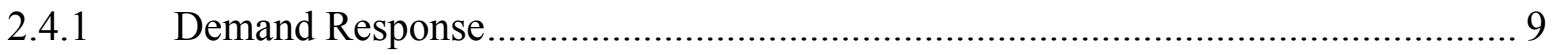

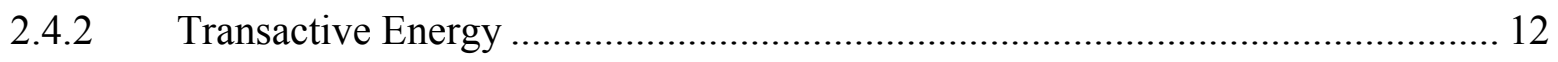

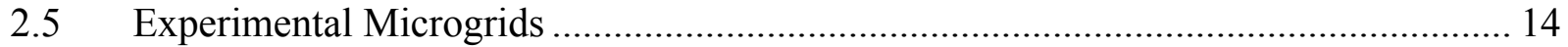

2.5.1 Centralized Experimental Microgrids ..................................................... 14

2.5.2 Autonomous Experimental Microgrids...................................................... 15

2.5.3 Agent Based Experimental Microgrids....................................................... 15

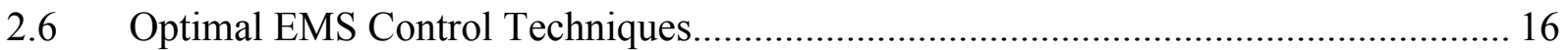

2.6.1 Mixed Integer Linear Programming …...................................................... 16

2.6.2 Mixed Integer Nonlinear Programming ...................................................... 17

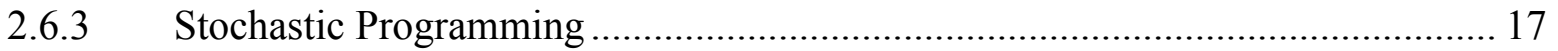

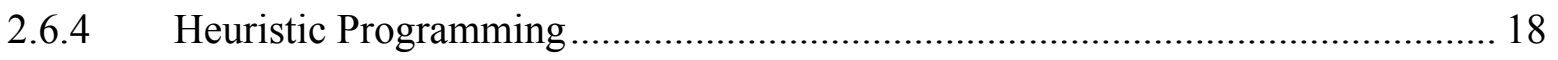

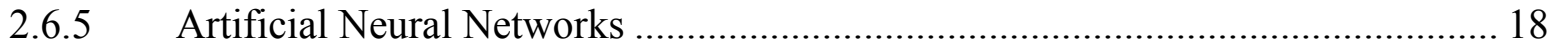

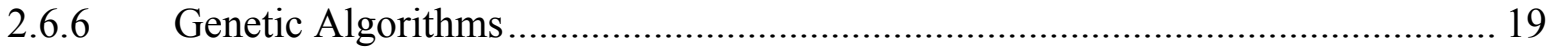

2.6.7 Model Predictive Control.......................................................................... 20

2.6.8 Literature Review Conclusions ................................................................. 23

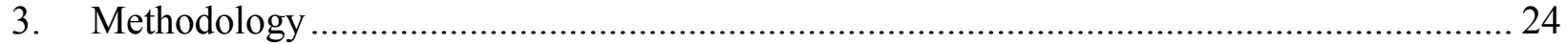

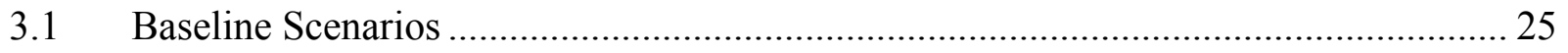




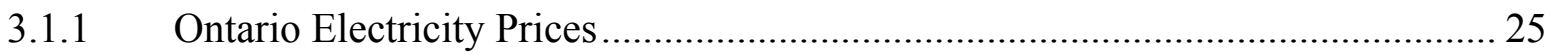

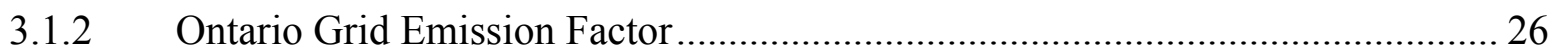

3.2 Deterministic Energy Management System Description.............................................. 29

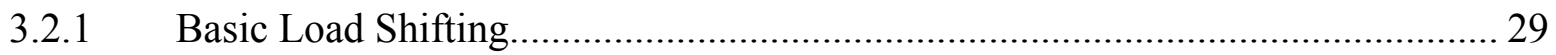

3.2.2 Load Shifting with Excess Charging .............................................................. 30

3.3 Optimized Battery Management System …………................................................. 30

3.4 Adaptive Energy Management System Description .................................................. 33

3.4.1 Machine Learning Battery Management System................................................ 33

3.4.2 Predictive Control Case Study Description ………………….......................... 35

3.4.3 Transactive Energy Management System Description .......................................... 38

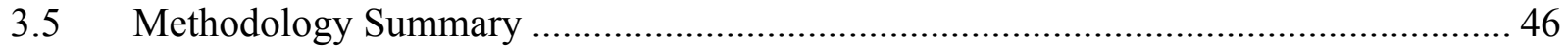

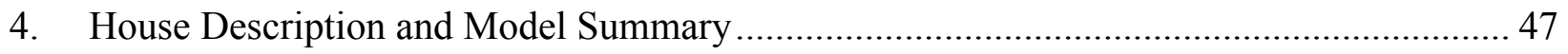

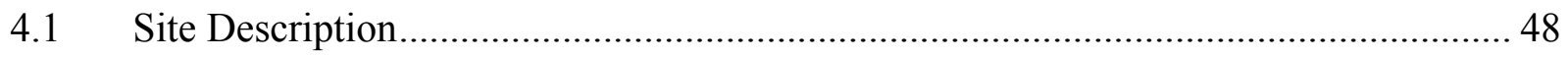

4.1.1 Data Management System ………………...................................................... 50

4.1.2 Mechanical and Renewable Energy Systems ………..................................... 50

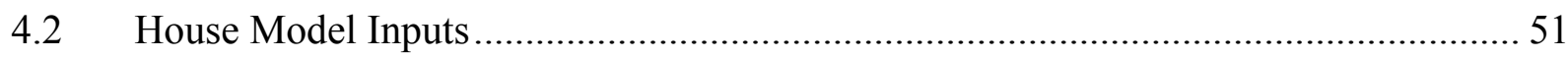

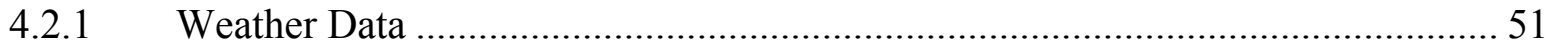

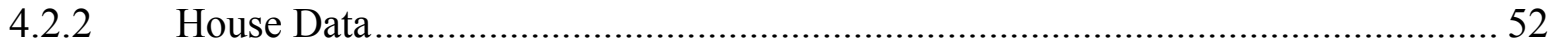

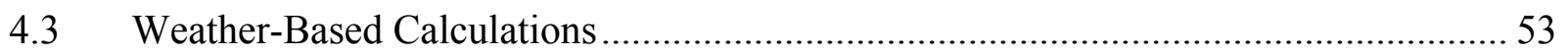

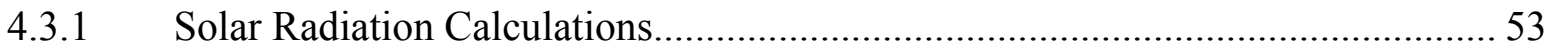

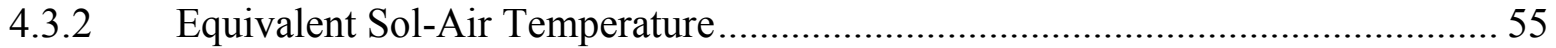

4.3.3 Convective Heat Transfer Coefficient .................................................................... 56

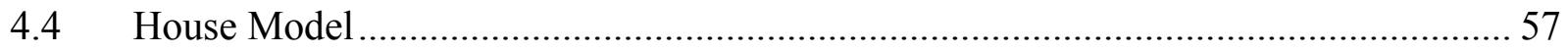

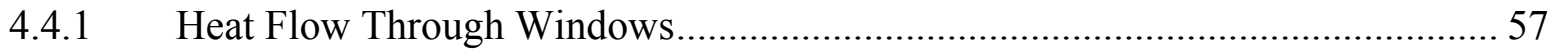

4.4.2 Solar Heat Gain Through Windows................................................................... 59

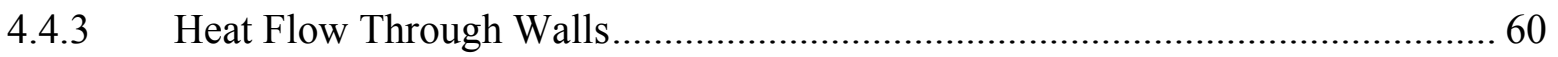

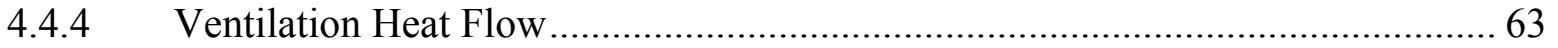

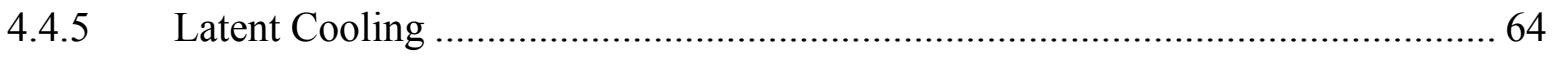

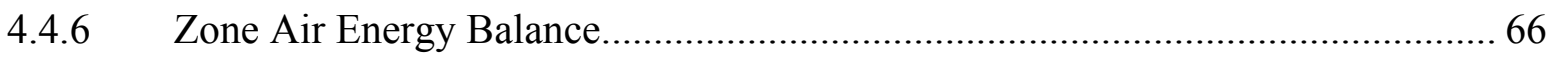

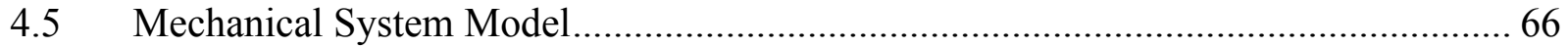

4.5.1 Heat Recovery Ventilator Model ............................................................................ 67 


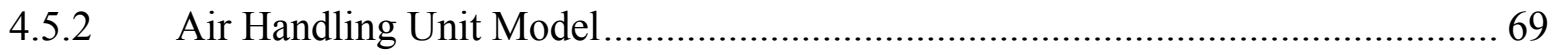

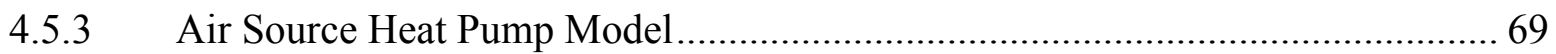

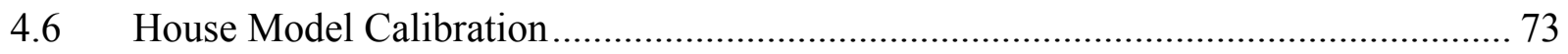

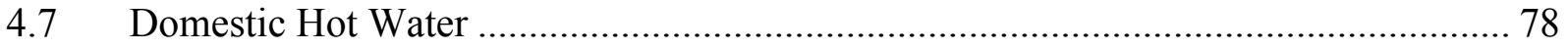

4.7.1 Domestic Hot Water Model Inputs ………….................................................. 79

4.7.2 Domestic Hot Water Model Procedure................................................................. 80

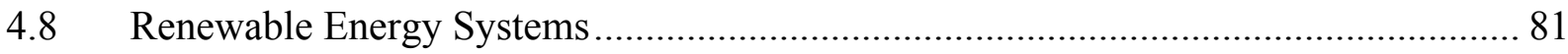

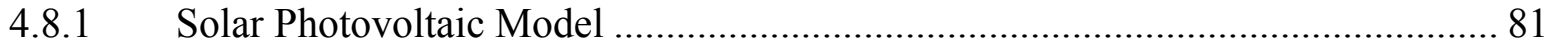

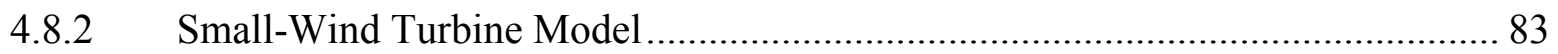

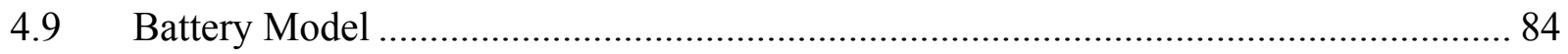

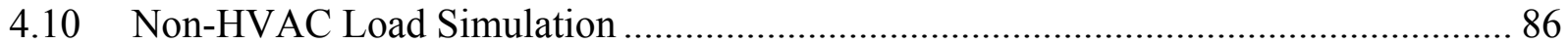

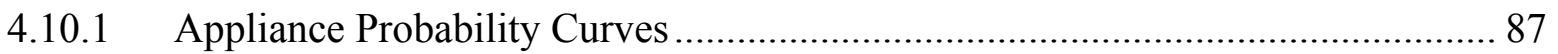

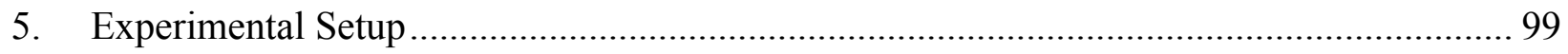

5.1 Load Shifting Experiment................................................................................. 99

5.2 Transactive Energy Management System Demonstration .......................................... 103

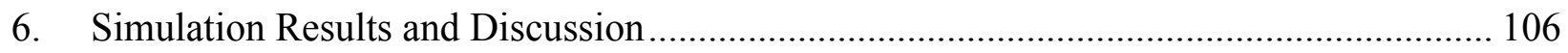

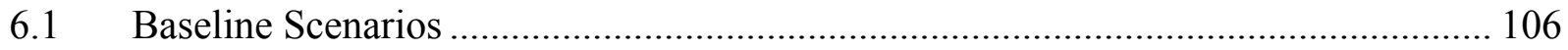

6.1.1 Space Conditioning Energy Consumption....................................................... 106

6.1.2 Domestic Hot Water Energy Consumption ........................................................ 106

6.1.3 Non-HVAC Load Energy Consumption............................................................ 108

6.1.4 Total Baseline Energy Consumption .............................................................. 109

6.1.5 Renewable Energy Generation ............................................................... 110

6.1.6 Baseline Energy Cost................................................................................ 111

6.2 Deterministic Energy Management Systems ………………………………......... 114

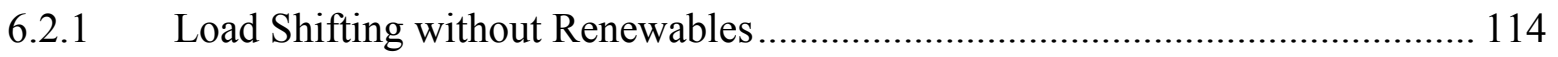

6.2.2 Basic Load Shifting with Renewables ................................................................. 119

6.2.3 Load Shifting with Excess Charging ............................................................ 122

6.2.4 Deterministic Energy Management Systems Summary …………………….... 125

6.3 Optimized Energy Management System …………................................................ 126

6.4 Adaptive Energy Management System Results ....................................................... 129

6.4.1 Machine Learning Battery Management Results............................................... 129 
6.4.2 Predictive Control Case Study Results …………….......................................... 132

6.4.3 Transactive Energy Management Simulation Results ....................................... 136

7. Experimental Case Study Results .......................................................................... 143

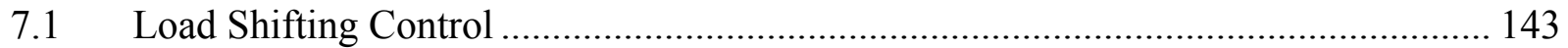

7.2 Transactional Energy Management System Demonstration....................................... 145

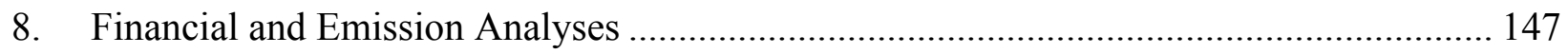

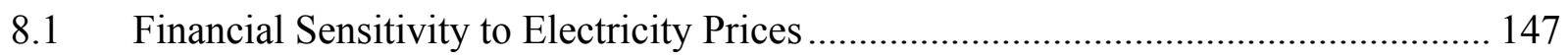

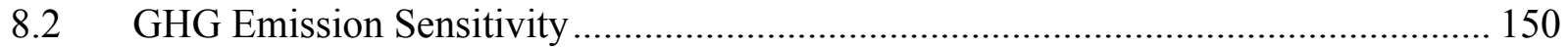

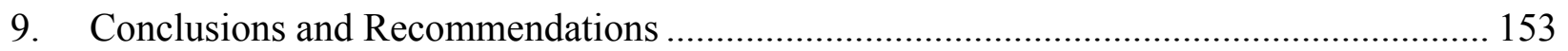

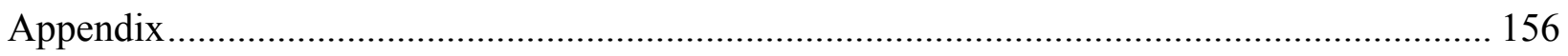

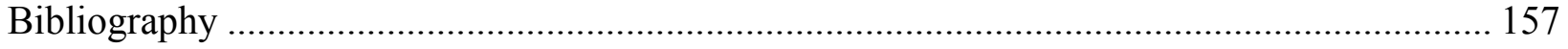




\section{List of Tables}

Table 2-1: National source energy conversion factors (NREL, 2007) ..................................... 6

Table 3-1: Toronto Hydro variable electricity prices as of May $1^{\text {st }}, 2017$ (OEB, 2017)............ 26

Table 3-2: Toronto Hydro fixed electricity prices as of May $1^{\text {st }}, 2017$ (OEB, 2017)................. 26

Table 3-3. Lifecycle emission factors of electricity generation sources (Moomaw et al., 2011). 28

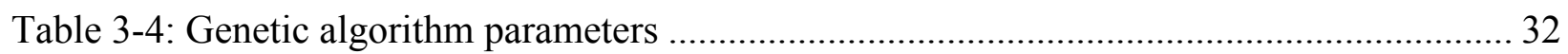

Table 3-5: Confusion matrix for battery control classifier using four weeks of data and medium

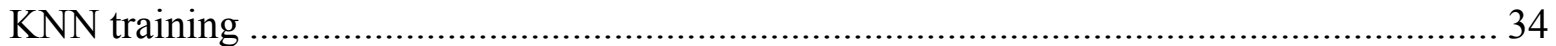

Table 3-6: Predictive ANN inputs and outputs.................................................................. 36

Table 3-7: Bid prices for solar PV in a TEMS ................................................................ 42

Table 3-8: Battery FIS control rules - Daytime................................................................... 45

Table 3-9: Battery FIS control rules - Nighttime .......................................................... 45

Table 4-1: Archetype Sustainable House structural features (Safa, Fung, \& Kumar, 2015)....... 49

Table 4-2: Archetype Sustainable House system summary................................................ 51

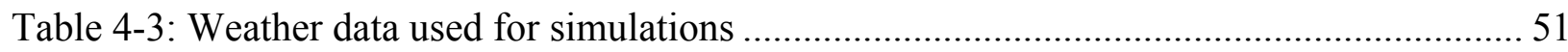

Table 4-4: Summary of fixed and calibrated parameters for the ASH-A model ....................... 52

Table 4-5: Orientation-specific ASH-A values (Alibabaei, 2016) ........................................ 52

Table 4-6: Relevant solar angles (Duffie \& Beckman, 2006) ................................................. 54

Table 4-7. Typical air change rates for different building types (Zhang, Fung, \& Jhingan, 2014)

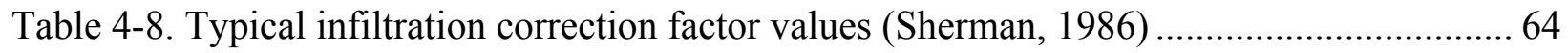

Table 4-9: ASHP technical specifications (Safa, Fung, \& Kumar, 2015) ................................ 69

Table 4-10: Long-term calibration heating and cooling outputs and targets ............................ 76

Table 4-11: Calibrated house model parameters ................................................................... 77

Table 4-12: A.O. Smith HPWH Specifications (A.O.Smith Enterprises Ltd., 2015)................. 78

Table 4-13: Summary of the three house configurations used for the simulation of non-HVAC loads (Armstrong, Swinton, Ribberink, Beausoleil-Morrison, \& Millette, 2009) ............... 86

Table 4-14: Annual appliance target characteristics (Armstrong, Swinton, Ribberink, Beausoleil-

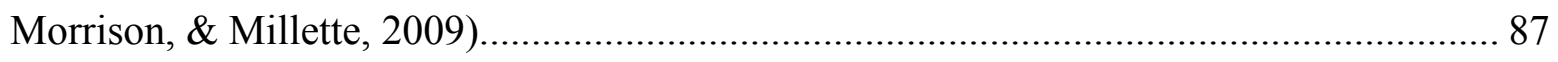

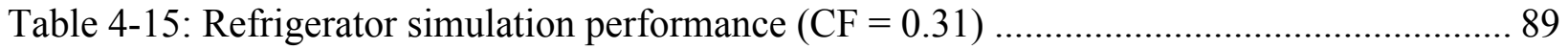

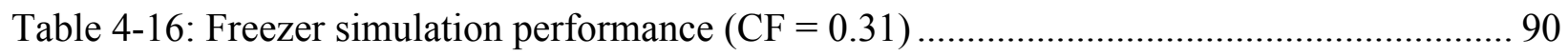




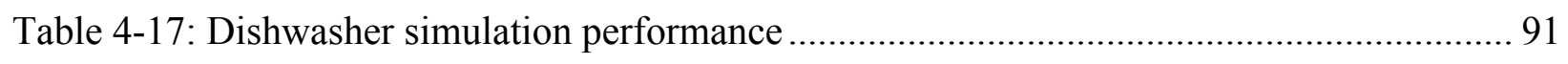

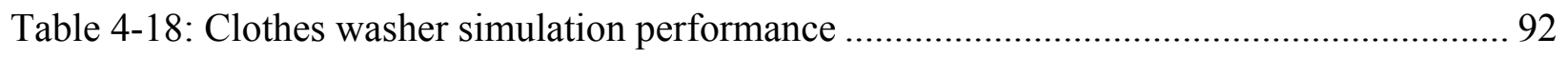

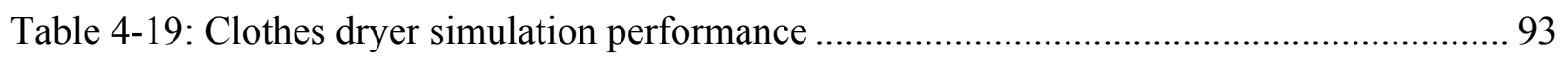

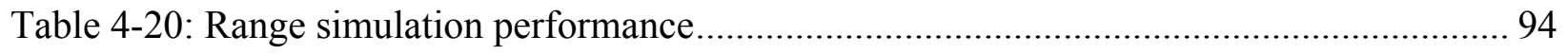

Table 4-21: List of miscellaneous appliances considered for simulation (U.S. Department of

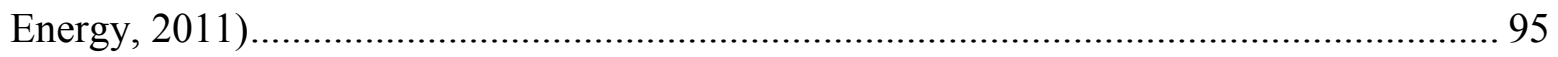

Table 4-22: Miscellaneous appliance simulation performance .............................................. 96

Table 4-23: Lighting load summary (Armstrong, Swinton, Ribberink, Beausoleil-Morrison, \&

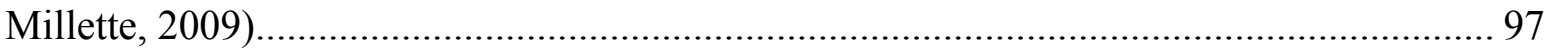

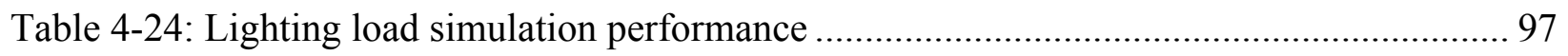

Table 6-1: Seasonal simulated heating and cooling energy consumption - Base case ............. 106

Table 6-2: AHU and HRV electricity consumption summary............................................ 106

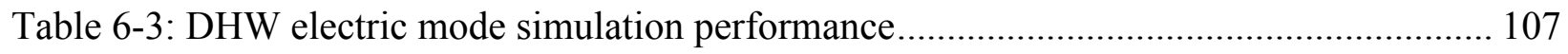

Table 6-4: DHW heat pump mode simulation performance.............................................. 107

Table 6-5: Changes in space conditioning electricity consumption when operating the water heater

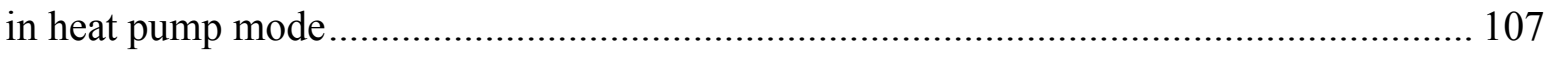

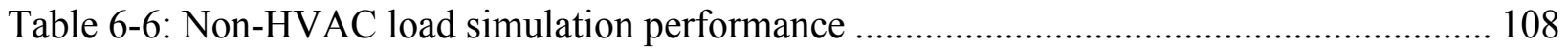

Table 6-7: Total simulated energy end-use summary........................................................ 109

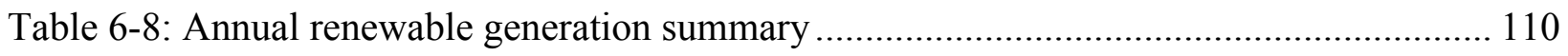

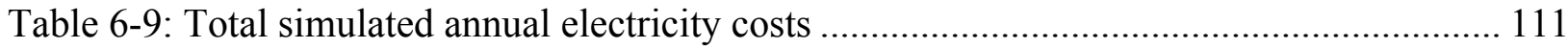

Table 6-10: Total simulated variable electricity costs associated with space conditioning and water heating

Table 6-11: Total simulated variable electricity costs for each of the three appliance usage characteristics.

Table 6-12: Renewable energy system capital costs as installed........................................ 114

Table 6-13: Annual load shifting savings summary for different load types ......................... 116

Table 6-14: Annual load shifting/zero-feedback savings summary for different load types and optimal battery size assuming a $5 \mathrm{~kW}$ zero-feedback PV system

Table 6-15: Annual load shifting/zero-feedback savings summary for different load types and optimal battery size assuming $5 \mathrm{~kW}$ PV and $6 \mathrm{~kW}$ wind turbine zero-feedback systems.. 120 
Table 6-16: Annual load shifting/excess energy storage savings summary for different load types and optimal battery size assuming a $5 \mathrm{~kW}$ PV system

Table 6-17: Annual load shifting with excess charging savings summary for different load types and optimal battery size assuming $5 \mathrm{~kW} \mathrm{PV}$ and $6 \mathrm{~kW}$ wind turbine systems.

Table 6-18: Distribution of battery state selection for the three different house types 126

Table 6-19: Cost comparison of zero-feedback operation to optimized battery management using $5 \mathrm{~kW} \mathrm{PV}$ and $6 \mathrm{~kW}$ wind turbine systems ...... 128

Table 6-20: Annual energy costs (and percent savings) associated with each house type using the MLS with different training methods and amounts of data 130

Table 6-21: Predictive ANN performance based on forecast or actual weather inputs. 134

Table 6-22: Summary of annual electricity savings and average zone temperatures associated with transactional energy-enabled temperature setbacks

Table 6-23: Energy cost and consumption comparison of the TEMS to normal operation 139

Table 6-24: Annual on-site renewable energy utilization using for a single house with a $4.08 \mathrm{~kW}$ PV system 141

Table 6-25: Traded price of solar energy in a TEMS 142

Table 7-1: Percentage of load shifted and days of operation 144

Table 7-2: Effect of temperature setback on power consumption 144

Table 8-1. Battery System Comparison for $55 \mathrm{kWh}$ Battery banks 147

Table 8-2: Annual savings associated with load shifting for a medium house without DGs based on different TOU prices 148

Table 8-3: Annual savings associated with basic load shifting for a medium house with a $5 \mathrm{~kW} \mathrm{PV}$ system based on different TOU prices. 148

Table 8-4: Annual savings associated with load shifting with excess charging for a medium house with a $5 \mathrm{~kW}$ PV system based on different TOU prices.

Table 8-5: Annual carbon emissions associated with grid electricity for different control strategies 150

Table 8-6: Emissions associated with load shifting for a medium house without DGs based nighttime charging time..... 151 


\section{List of Figures}

Figure 2-1: The evolution of the smart grid (IEA, 2011) ...................................................... 4

Figure 2-2: Net-zero energy site boundary (DOE, 2015) .............................................. 5

Figure 2-3: Example hybrid microgrid configuration (Unamuno \& Barrena, 2015) .................. 7

Figure 2-4: General optimizing microgrid EMS (Minchala-Avila, Garza-Castañón, Vargas-

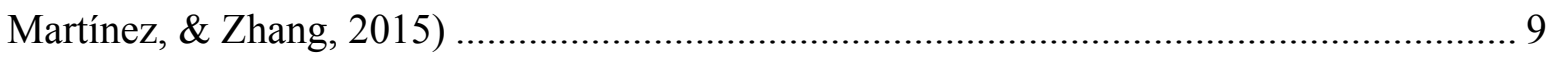

Figure 2-5: An example demand response period effect on household power consumption ....... 10

Figure 2-6: Illustration of Biding Strategy of Thermostat in Cooling Mode in Transactive Energy

Network (Katipamula, Hatley, Hammerstrom, Chassin, \& Pratt, 2006).......................... 13

Figure 2-7: An example of an ANN used for solar PV generation predictions ......................... 19

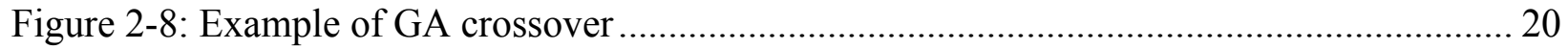

Figure 2-9: Model Predictive Control Concept ............................................................... 21

Figure 3-1: Ontario TOU pricing schedule including electricity prices and total cost per kWh in

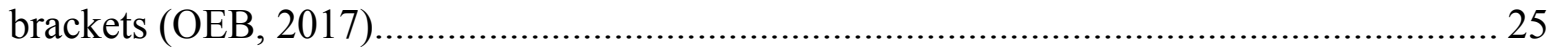

Figure 3-2: Ontario Electricity Grid Generation by Fuel Type (IESO, 2016) .......................... 27

Figure 3-3: Hourly averaged emission factors based on 2015 IESO generation data ................ 28

Figure 3-4: Example of the resulting load of a chromosome evaluation................................... 32

Figure 3-5: ROC curve for classifier training using one month of data under medium KNN training

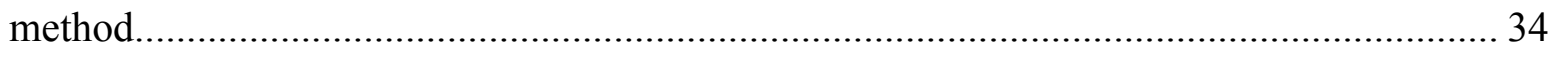

Figure 3-6: Training validation accuracy of the MLS for each month of the year .................... 35

Figure 3-7: An example of supply and demand evaluation in the Energy Marketplace.............. 40

Figure 3-8. Sample ASHP bid curve relative to different energy prices. ................................ 41

Figure 3-9: Battery FIS input membership function - Battery capacity fraction ..................... 43

Figure 3-10: Battery FIS input membership function - Net load fraction.............................. 43

Figure 3-11: Battery FIS output membership function - Bid price ...................................... 44

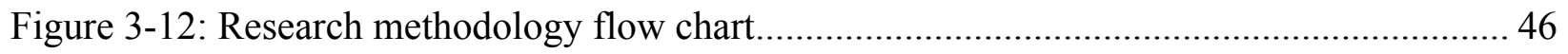

Figure 4-1: The Archetype Sustainable House A (left) and House B (right) at the Living City

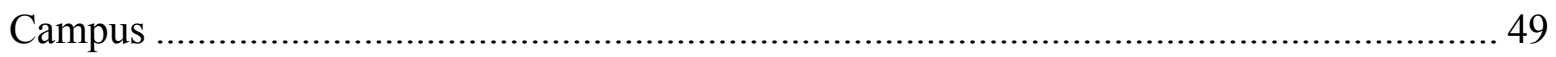

Figure 4-2: Data Management System hierarchy (Saxena, 2017) ......................................... 50

Figure 4-3: Relevant solar calculation angles (Duffie \& Beckman, 2006).............................. 54

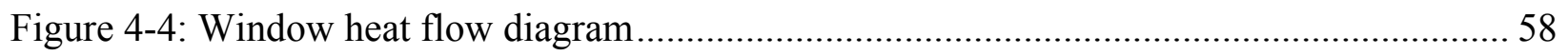


Figure 4-5: Wall heat flow diagram

Figure 4-6: ASHRAE summer and winter thermal comfort zones (Howell, Coad, \& Sauer, 2013)

Figure 4-7: House A mechanical system power consumption. 67

Figure 4-8: Heat recovery ventilator ANN performance 68

Figure 4-9. ASHP experimental power consumption in both first (right) and second stages (left) (Safa, Fung, \& Kumar, 2015) 70

Figure 4-10: ASHP experimental COP as a function of outdoor temperature (Safa, Fung, \& Kumar, 2015) 71

Figure 4-11: COP in cooling mode as a function of outdoor temperature (Safa, Fung, \& Kumar, 2015) 72

Figure 4-12: ASHP cooling provided as a function of outdoor temperature ........................... 73

Figure 4-13: Ambient conditions over cool-down test period ............................................. 74

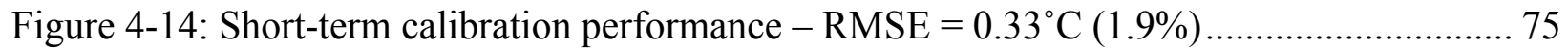

Figure 4-15: Annual simulated heating and cooling energy output..................................... 76

Figure 4-16: A.O.Smith power consumption in heat pump mode over a one-week period ......... 79

Figure 4-17: Monthly average water inlet temperature (Amirirad, 2016) .............................. 80

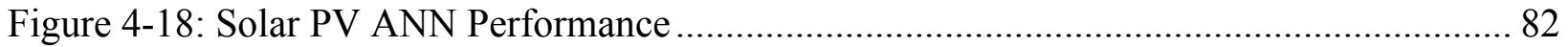

Figure 4-19: Solar PV ANN Performance on sample hourly dataset $\left(\mathrm{R}^{2}=0.9906\right) \ldots \ldots \ldots \ldots \ldots \ldots . . . . . . .83$

Figure 4-20: Experimentally determined small wind turbine power curves (SWCC, 2016)....... 84

Figure 4-21: Refrigerator chance factor determination. Calibrated value $-\mathrm{CF}=0.31 \ldots \ldots \ldots \ldots . .89$

Figure 4-22: Sample refrigerator daily averaged load profile and probability curve ................. 90

Figure 4-23: Sample dishwasher daily averaged load profile and probability curve (medium energy house) 91

Figure 4-24: Sample clothes washer daily averaged load profile and probability curve (medium energy house). 92

Figure 4-25: Sample clothes dryer daily averaged load profile and probability curve (medium energy house). 93

Figure 4-26: Sample range daily averaged load profile and probability curve (medium energy house) 94 
Figure 4-27: Sample aggregated miscellaneous appliance daily averaged load profile and

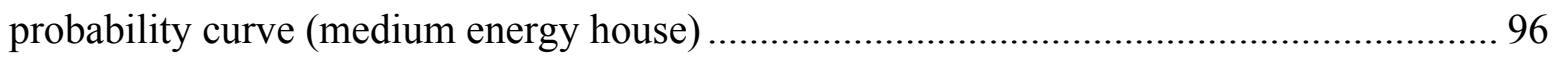

Figure 4-28: Sample lighting load daily averaged load profile and probability curve (medium

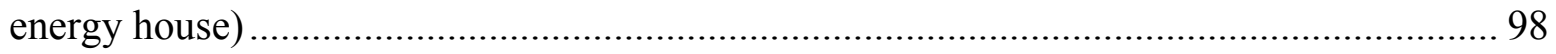

Figure 5-1: Diagram of experimental load shifting setup .................................................. 99

Figure 5-2: State diagram of battery control program ....................................................... 102

Figure 5-3: HPWH bid curve for Marketplace demonstration ............................................ 104

Figure 6-1: Sample hourly averaged simulated non-HVAC load profiles ............................. 108

Figure 6-2: Total simulated energy end-use percentage breakdown .................................... 109

Figure 6-3: TOU consumption distribution for normal house operation .............................. 110

Figure 6-4: TOU consumption distribution for the medium household using zero-feedback

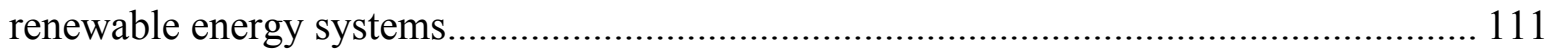

Figure 6-5: Cost comparison of zero-feedback renewable generation systems....................... 113

Figure 6-6: Annual load shifting cost savings for load shifting based on battery size for the lowenergy consumption house

Figure 6-7: Annual load shifting cost savings for load shifting based on battery size for the medium-energy consumption house

Figure 6-8: Annual load shifting cost savings for load shifting based on battery size for the highenergy consumption house 116

Figure 6-9: Optimal battery bank size for varying household loads based on basic load shifting control and number of days depleted annually 117

Figure 6-10: Changes in annual consumption using load shifting without renewables ............ 118

Figure 6-11: Sensitivity of battery bank size using basic load shifting to PV array size based on 5 days of annual depletion

Figure 6-12:Battery bank size with and without a wind turbine based on 5 days of depletion annually using basic load shifting.

Figure 6-13: Sensitivity of battery bank size using load shifting with excess charging to PV array size based on 5 days of annual depletion 124

Figure 6-14: Battery bank size with and without a wind turbine based on 5 days of depletion annually using load shifting with excess charging 124 
Figure 6-15: Annual energy consumption for different house types and different load shifting procedures

Figure 6-16: Hourly distribution of battery state selection for the medium-consumption house 126

Figure 6-17: Annual energy consumption comparison between normal operation, load shifting, and optimized battery management

Figure 6-18: Annual cost savings associated with training the MLS with different data sets.... 129

Figure 6-19: Annual energy consumption comparison between MLS's and baseline cases ...... 131

Figure 6-20: HVAC electrical load ANN based on hidden layer size for use in predictive control

Figure 6-21: Solar PV generation ANN based on hidden layer size for use in predictive control 132

Figure 6-22: HVAC ANN output using weather forecast and actual weather data compared to "actual" (simulated) power consumption. 133

Figure 6-23: Solar PV ANN output using weather forecast and actual weather data compared to "actual" (simulated) generation 133

Figure 6-24: Comparison of EMS cost across the 10-day forecast period 134

Figure 6-25: ANN-predicted versus actual ASHP Power consumption. 135

Figure 6-26: Electrical loads of four houses using a TEMS with and without a DR signal....... 137

Figure 6-27: Thermostat bid curves representing different thermal comfort preferences ......... 138

Figure 6-28: An example of the TEMS using flexible loads to align consumption with generation 141

Figure 7-1: Mid- and on-peak ASHP consumption, generation, and battery bank output. 143

Figure 7-2: Energy Marketplace and Agent state diagrams relative to the DDS layer. 145

Figure 7-3: Example of the removal of the solar PV agent during the Energy Marketplace demonstration. 146

Figure 8-1: Historical Ontario electricity TOU rates, not including marginal costs (OEB, 2017) 149 


\section{Introduction and Objectives}

The increasing world energy demand requires the active development of sustainable energy systems in all sectors. Of the many contributing factors, the buildings sector's role in energy consumption is of great interest. In 2012, residential buildings accounted for $22 \%$ of the United States' energy consumption (DOE, 2012), and 17\% of Canada's energy consumption (NRCan, 2014). Excluding electricity consumption, the building sector also contributed to $11 \%$ of Canada's total greenhouse gas (GHG) emissions in 2012 (NRCan, 2014). Scenarios developed by the United Nations Framework Convention on Climate Change project building energy demand is expected to rise by 15\% from 2013-2030; the majority this increase will be supplied by electricity (IEA, 2015). Increased effort towards developing sustainable practices in the residential sector will therefore have a substantial impact on reducing world energy demand and GHG emissions.

This information is not lost on the world - increasing concern towards carbon emissions and climate change is already changing the ways in which energy is produced, distributed and consumed. The rise of renewable energy is leading to more distributed generation - moving away from large, centralized generation facilities towards smaller generators spread out over a large area. Not only is electricity generation changing, but so is the way it is getting used; historical data shows that while the number of households and businesses in Ontario is increasing, the energy intensity of buildings is decreasing due to conservation and efficiency measures (Ontario Ministry of Energy, 2013).

Along with changes in the way energy is being generated and consumed comes the need for smarter energy management techniques on both the macro and micro scales. These technologies are already in use today, and can range in both size and complexity - from individual smart thermostats in homes to widespread demand response programs across a local distribution network.

As technology advances, the lines between the consumer and utility are becoming more blurred. Households with distributed generation (DG) are no longer viewed simply as consumers, but as prosumers who both consume and produce energy. This creates a need for greater interaction between the consumer and utility - interactions enabled by smart grid technologies. 
These technological revolutions cannot be implemented in an ad hoc manner - their effective management will be a significant task. The introduction of residential energy storage adds another dimension to the management problem. Household batteries can be used for a variety of purposes:

they can be used as dispatchable loads and capacity if managed by utility companies, or used by homeowners as backups during blackouts, to store excess renewable electricity generation, and much more.

This is the primary topic of interest of this work: the effective management of residential battery energy storage in conjunction with loads and on-site renewable energy generation. This work explores just a few of this vast number of battery and energy management possibilities.

\subsection{Objectives}

This research involves the investigation of simulated and experimental energy management systems (EMSs). Throughout this work, EMS is used as an umbrella term to refer to any software responsible for decision making and control of energy consumers and/or producers in a home. The detailed objectives of this work are as follows:

- Identify different EMSs whose performance may be investigated, through reasonable means, by either simulation or experimental trials.

- Develop a simulated residential building model by which the selected EMSs may be tested and compared on the same basis.

- Perform experimental trials when possible to validate claims made in simulation work.

- Compare the different EMSs and identify the advantages, disadvantages, and potential practical applications of each system.

It is the author's opinion that the ease of implementation of an EMS is the most important aspect of its design; an EMS should be "plug-and-go", in the sense that the average homeowner should be able to install and commission one in a reasonable amount of time, without frustration. Complications involved with the setup and operation of an EMS will act as a significant barrier to their uptake for residential purposes. Therefore, a strong emphasis has been made on the ease of implementation of each of the EMSs investigated. 


\section{Literature Review}

Various energy management systems are already being implemented in homes across the world today; it is not uncommon to find a programmable or "smart" thermostat in somebody's home. However, EMSs can have a much greater reach than only the control of the heating and cooling systems of a home. With the advent of the internet, an unprecedented communication boom is occurring. Most people take daily advantage of the inter-personal communication brought about by the internet, but there are a great number of technical applications that can be improved through inter-system communication. With respect to the energy sector, this future, highly-communicative technology is referred to as the Smart Grid - an EMS on the largest scale.

\subsection{The Smart Grid}

Worldwide electricity demand is increasing at an unprecedented rate; projections show $150 \%$ growth under IEA's ETP 2010 Baseline Scenario (IEA, 2011). This dramatic increase in demand brings forth a critical need to improve upon the state of the electrical distribution system on a global scale. The current electricity distribution grid in most developed countries is outdated; it was designed to deliver energy in a single direction - from centralized electricity generation to the consumer - with minimal communication on the consumer's end.

Our ability to exchange information is evolving at an extraordinary rate. The Smart Grid of the future will use information transactions to control the flow of electricity, through intelligent decision making processes, taking both generators and consumers into consideration (Gelazanskas \& Gamage, 2014). The adoption of the Smart Grid is not a transformation that will occur over night; rather, it will be an evolutionary process that develops over time. Figure 2-1 displays the progression of the electricity grid towards the future Smart Grid. 


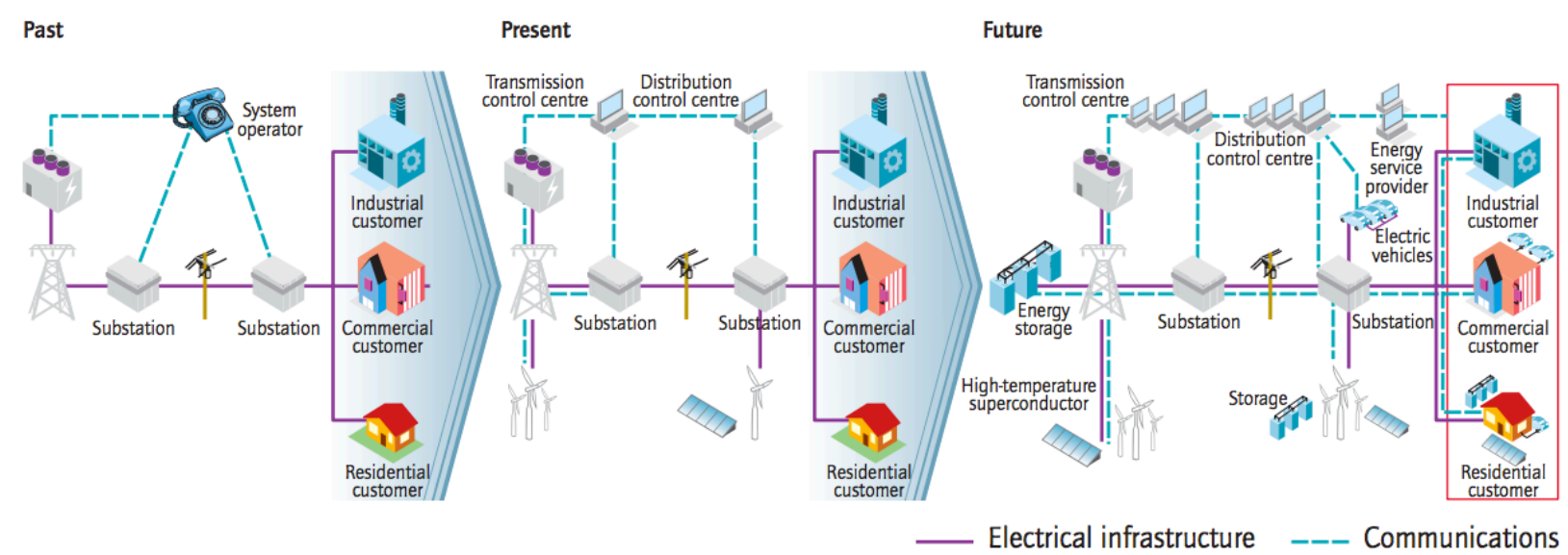

Figure 2-1: The evolution of the smart grid (IEA, 2011)

The Smart Grid will have many benefits over the current distribution grid. Along with increased reliability and consumer participation, it will be able to accommodate renewable distributed generators (DGs) and energy storage technologies. Energy storage solves the intermittency problem with renewables, allowing sustainably generated energy to be utilized at any time of day. The current state of electricity grids around the world is ill-equipped to deal with the variability and unpredictability of DGs, particularly solar and wind power. The issue of DG integration will require many different smart decision-making systems of varying size and planning capabilities. (IEC, 2012). While the Smart Grid refers to the state of the electricity grid as a whole, EMSs are responsible for the decision-making and control of smaller subsets of the overall system. EMSs will be the means of deployment for Smart Grid-enabled technologies and processes.

The distributed nature of renewable energy generation allows electricity generation to be located nearby the consumer, or even on-site. This will allow buildings to generate their own power, reducing their reliance on the large-scale distribution grid, with the potential to be net producers of energy.

\subsection{Net-Zero Energy Buildings}

Net-Zero Energy Buildings (NZEB) are gaining increasing interest as means of effectively eliminating building energy consumption. A NZEB consumes as much energy as it produces, through the interaction of onsite renewable energy systems and the utility grid (Marszal et al., 
2011). Although the time basis for net-zero energy calculations has varied in the past, more recently a "common definition" for NZEBs specifies that the site's delivered energy must be less than or equal to the exported renewable energy on an annual basis (DOE, 2015). The concept of net-zero energy may be applied to a single building, or be expanded to encompass an entire community, based on how the boundary is defined. Figure 2-2 illustrates the site boundary around a NZEB or community.

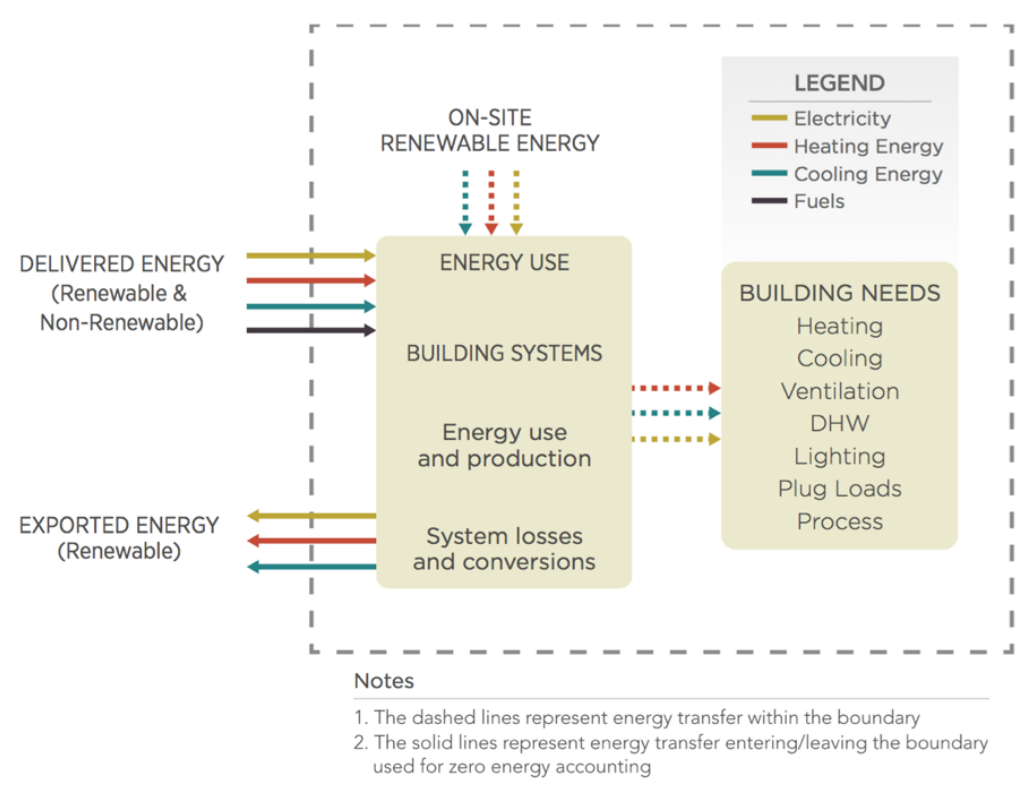

Figure 2-2: Net-zero energy site boundary (DOE, 2015)

In order to calculate the net energy transfer across the site boundary, source energy calculations must be performed. Source energy takes into account energy consumed through the extraction, processing, and transportation of the primary fuel used to produce the electricity or heat. Source energy across a building boundary may be calculated in Equation 2-1 (DOE, 2015):

$$
E_{\text {source }}=\sum_{i=0}^{n}\left(E_{d e l, i} * r_{d e l, i}\right)-\sum_{i=0}^{n}\left(E_{\text {exp }, i} * r_{\text {exp }, i}\right)
$$

Where the subscripts $d e l$ and exp represent delivered and exported energy respectively, and $r$ is the source energy conversion factor. Based on this calculation, a building would be considered 
Net-Zero if $E_{\text {source }} \leq 0$. General source energy conversion factors are shown in Table 2-1. It should be noted that imported and exported electricity are given the same conversion factor in calculations because they are subject to the same distribution losses once outside of the site boundary (DOE, 2015).

Table 2-1: National source energy conversion factors (NREL, 2007)

\begin{tabular}{|l|c|}
\hline & $\begin{array}{c}\text { U.S. } \\
\text { National }\end{array}$ \\
\hline Transportation \& Distribution Losses & $9.90 \%$ \\
\hline Fossil Fuel Energy & 2.500 \\
\hline Renewable Energy & 0.177 \\
\hline Total Energy & 3.365 \\
\hline
\end{tabular}

Various projects aim to reduce energy demand for entire residential communities. One such community in the Southwest United States aims to reduce peak electricity demand by $65 \%$ through the integration of rooftop solar PV arrays and battery energy storage systems (Boehm, 2011).

NZEBs take advantage of high-efficiency appliances and heating, ventilation, and air conditioning (HVAC) systems. Heat pumps are of particular interest for NZEBs. This type of heating and cooling appliance can come in many forms, all of which function by moving heat from one place to another. The most common forms for houses are air- and ground-source heat pumps (ASHPs and GSHPs). They can heat a home by transferring heat from the air or ground, respectively, into the space of a building, and cool the same space by operating in reverse. While not all heat pumps are electrically driven, both ASHPs and GSHPs are all-electric systems. These are of particular interest with regards to NZEBs, as they can be complemented by on-site renewable generation.

While an EMS does not require a NZEB to run, the building industry is moving towards the development of net- (or nearly-) zero energy buildings. These types of buildings will house the EMSs of the future, and as such, special consideration should be taken in the design of an EMS for the requirements of NZEBs. 


\subsection{Microgrids}

NZEBs and communities can be connected to the smart grid through what is known as a microgrid. A microgrid is a component of the Smart Grid containing DGs, energy storage, and loads, with the capability of operating in conjunction with or independently from the main utility grid. The term islanding is used to describe a microgrid disconnecting from the main grid and operating autonomously. A microgrid is connected to the main distribution grid at what is called the pointof-common-coupling (PCC), which is typically on the low-voltage side of the local substation transformer (Lidula \& Rajapakse, 2011). A microgrid can help bring sustainable, reliable energy to areas that do not have typical access to a main distribution grid, potentially providing additional social and economic benefits to isolated areas (IEA, 2015). The size of a microgrid may vary greatly; it may range from a few houses in size, to a community, to an entire town.

The distributed energy resources in a microgrid may include a wide variety of technologies. Examples include solar photovoltaics (PV), solar thermal, wind turbines, fuel cells, combined heat and power (CHP), diesel generators, or small-scale hydroelectric turbines. It is worth noting that among these technologies, some generate DC electricity (PV, fuel cells), while others generate AC electricity (turbines, generators). Because of this, microgrids also require inverters/converters in order to accommodate its loads and generation sources. Hybrid AC/DC microgrids, containing both AC and DC components, are of the most interest in literature (Unamuno \& Barrena, 2015). Figure 2-3 displays an example of a general microgrid configuration.

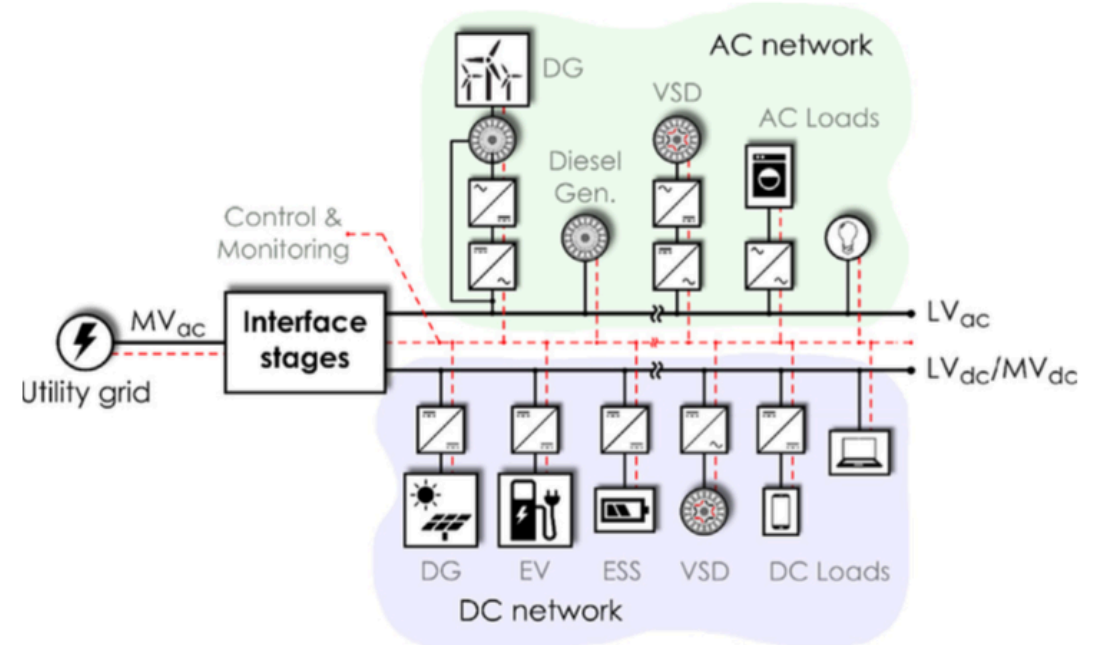

Figure 2-3: Example hybrid microgrid configuration (Unamuno \& Barrena, 2015) 
Energy storage devices are a key component of microgrid operation; they allow islanding and load smoothing when connected to the main distribution grid. They can also smooth out the generation of intermittent DGs. A variety of energy storage devices exist with potential microgrid applications. Of particular interest are batteries, flywheels, and super capacitors. These storage devices may all be used together in a single microgrid as complements to each other, reducing excessive strain on a single unit.

Increasing interest in plug-in electric vehicles $(\mathrm{EVs})$ will play an integral role in future smart buildings and communities. Not only do EVs have the potential to reduce transportation-associated greenhouse gas (GHG) emissions, they may also act as a form of energy storage: vehicle-to-grid (V2G) based storage. An EV charger with bidirectional capabilities can allow a homeowner to utilize their EV battery as a tool for peak load shifting and energy cost reductions. V2G storage has significant advantages over conventional vehicle charging. It has also shown potential as a more efficient means of renewable energy utilization, when compared to stationary energy storage, depending on travel patterns and the accessibility of workplace chargers (Tarroja, Zhang, Wifvat, Shaffer, \& Samuelsen, 2016). Simulations have also demonstrated EV energy storage methods capable of reducing electricity costs by an average of 11\% (Onda, Yamamoto, Takeshit, Okamoto, \& Yamanaka, 2014).

In order to operate its various components effectively, a microgrid requires a control strategy to manage its distributed loads, storage devices, and loads. While an EMS does not need to be a component of a microgrid for operation, much of the research regarding microgrid control involves EMSs. It is therefore useful to view EMS control and microgrid control as synonymous for the purposes of this review, since microgrids are composed of all the components for which an EMS is responsible. This interpretation does however neglect certain important control aspects of microgrids, such as voltage/frequency control and anti-islanding. However, these aspects of microgrid control require significant electrical engineering, and are therefore out of the scope of this work. Moving forward, it should be noted that any mention of "microgrid control" refers specifically to the EMS used within a microgrid. 


\subsection{Energy Management Systems}

Effective EMSs will play a significant role in the development of the smart grid. Innovative EMS designs should be composed of plug-and-play components (in terms of both hardware and software), allow increased functionality of controlled components, and support services such as historical databases and user interfaces (Zhang, Mao, Ding, \& Chang, 2008). The objective of an effective EMS is often to minimize a cost function. This makes the development of new EMSs attractive to both consumers - by reducing utility cost - as well as the utility provider - through the development of more robust distribution systems. The optimization of an EMS is key to unlocking its full potential. Figure 2-4 highlights the components of a general microgrid EMS. There is no standard for the design of an EMS, and it is therefore an area of great interest for the development of the smart grid. They may use a variety of tools and techniques to optimize energy management.

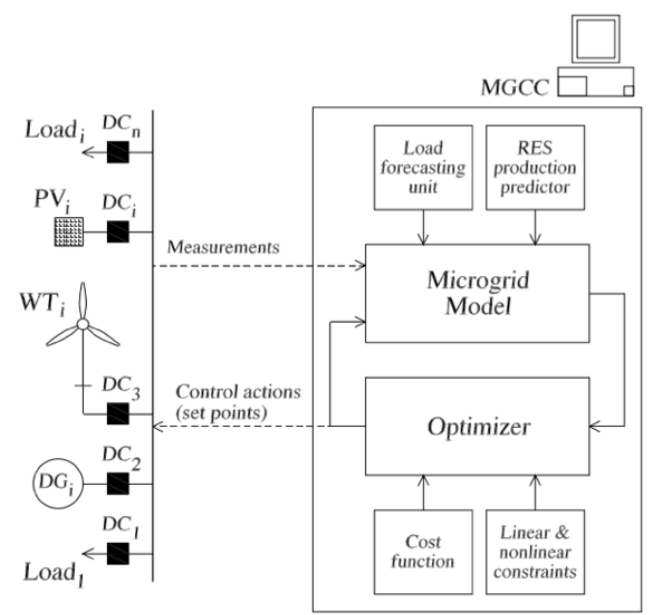

Figure 2-4: General optimizing microgrid EMS (Minchala-Avila, Garza-Castañón, VargasMartinez, \& Zhang, 2015)

\subsubsection{Demand Response}

Demand response (DR) strategies will play an integral role in smart building energy management, and the evolution of the Smart Grid. They will aid in facilitating the effective addition of DGs to the electrical distribution grid (CEA, 2013). Through DR strategies, the smart grid will have the capability to manage consumer energy consumption patterns based on incentives and disincentives (Siano, 2013). The concept of demand response is illustrated in Figure 2-5 


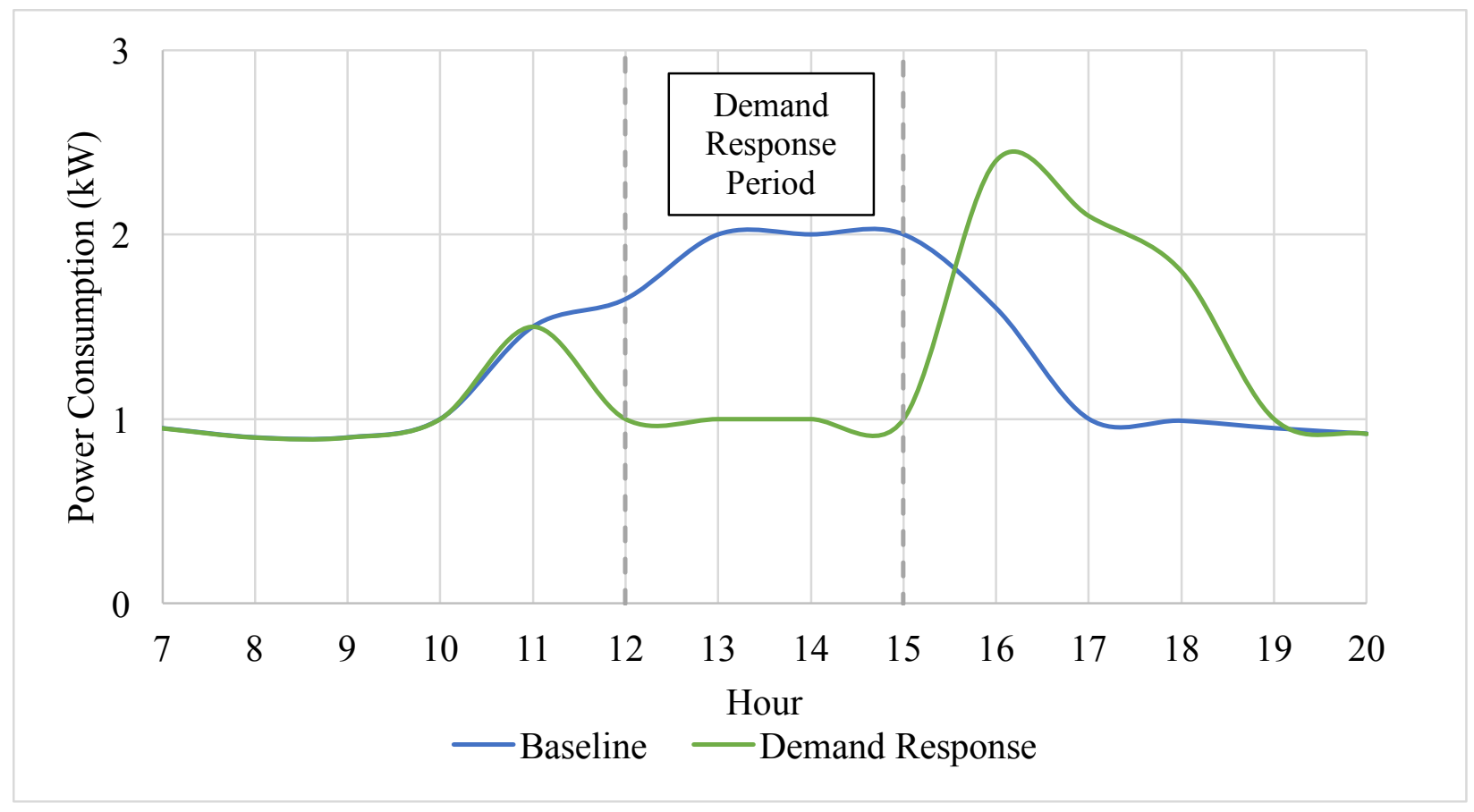

Figure 2-5: An example demand response period effect on household power consumption

One form of DR utilizes time-of-use (TOU) electricity pricing information for decision making. An internet-scale distribution model has been shown to realize $12-32 \%$ electricity cost savings using optimized load shifting algorithms (Mathew, Sitaraman, \& Shenoy, 2014). Load shifting is a form of DR that consists of moving elastic energy demand to off-peak times through price-based signaling.

Local Distribution Companies (LDC) have already implemented real-world DR programs. In Ontario, Canada, the Independent Electricity System Operator (IESO) ran a large-scale Demand Response Pilot Project. Through this project, the participating resources totaling $80 \mathrm{MW}$ in size, were required to modify their electricity consumptions for a minimum of 100 hours per contract year (IESO, 2015). The program uses five-minute and hourly load tracking, as well as unit commitment to better manage and understand how DR can help benefit electricity requirements. Other programs such as Toronto Hydro's DR3 aim to engage large-scale businesses, with peak demands greater than $50 \mathrm{~kW}$, in reducing their electricity demand at peak times through financial incentives (Toronto Hydro, 2012). 
Residential-scale DR initiatives have also been implemented in the real world. In Ontario, Canada, a group of LDCs have implemented the peaksaver PLUS program (Poulad, Fung, He, \& Colpan, 2016). This program involved signaling participating residential thermostats to pause during certain summer peak hours, halting air conditioner operation (Hydro Ottawa Ltd., 2016). With participation in the range of 28,000 - 35,000 residential units per LDC, all participating LDCs have now closed registration and halted program expansion (Powerstream, 2016).

DR can be complemented well by renewable energy and storage. Experimental studies on a single unit solar house in Madrid has shown weekday energy consumption savings of $1.2-2.2 \mathrm{kWh}$ utilizing DR with PV generation and battery storage (Castillo-Cagigal et al., 2011). Neural network controllers utilizing active DR with PV energy generation also demonstrate the potential for implementation in the residential sector (Matallanas et al., 2012).

A component of DR is load scheduling. This involves load categorization, which typically sorts home appliances into three categories (Liu, Ivanescu, Kang, \& Maier, 2012). Those are appliances having:

i) real time energy consumption modes, relating directly to consumer behavior (e.g. microwaves and printers);

ii) periodic nonreal-time energy consumption modes, in which energy consumption is periodic and fluctuating while in use (e.g. refrigerators); and,

iii) nonperiodic nonreal-time energy consumption modes, which are similar to (ii) but have a task deadline (e.g plug-in electric vehicles and pool pumps).

Load scheduling also requires load prioritization, which must be dynamically allocated based on appliance status (Liu, Ivanescu, Kang, \& Maier, 2012). Load scheduling algorithms for smart building energy management with renewable energy sources have been shown to have the potential of reducing electricity costs paid to the utility by $41-94 \%$ (Radhakrishnan \& Selvan, 2014). A case study on dynamic load management has shown a successfully imposed load limit of $1500 \mathrm{~W}$ during demand response events (Fernandes, Morais, Vale, \& Ramos, 2014).

DR does not have to mean sacrificing thermal comfort at times when peak reduction is requested; demand-side management models with consumer preference for residential smart grids have shown success in energy cost reduction using peak-to-average ratio constraints (Liu et al., 2014). 
One study shows that smart grid incentives, in conjunction with fuzzy logic controllers and wireless sensors, can produce residential energy savings of $14.5 \%$ without sacrificing thermal comfort (Keshtkar, Arzanpour, Keshtkar, \& Ahmadi, 2015).

\subsubsection{Transactive Energy}

While direct load control strategies considering TOU pricing schemes have had success incentivizing consumers at peak hours, empirical evidence shows that they do little to address energy consumption at non-critical hours (Faruqui \& Sergici, 2010). In order to address this shortfall, yet maintain fast demand response and consumer comfort, demand response methods based on microeconomic theories have been proposed for electrical system applications (Schweppe et al., 1980). The concept of applying a free market economic model to energy supply and demand is known as Transactive Energy.

A transactional energy network is comprised of energy control systems that conduct live energy transactions through a digital market in which consumers and producers bid and negotiate energy exchanges (PNNL, 2014). The transactional energy framework allows for decentralized, selforganizing control agents that communicate through local digital infrastructure. This type of energy system shows significant potential for future smart building control.

The concept of transactional energy has been extensively researched by Pacific Northwest National Laboratories (PNNL). They show that this type of energy network can be easily imagined through example of a thermostat. In a transactive energy network, a thermostat would bid on an amount of energy at a calculated unit dollar amount. The dollar amount would be based on dynamic factors such as current occupancy levels, thermal comfort margins, or deviation from zone set point. The transactive energy marketplace takes all participating loads and generation capacity into consideration to determine the price at which energy is traded - the market clearing price. This decision can be made externally by the utility, or internally based on an exchange of internal services through smart building implementation. Thermostat bids are then granted or turned down based on whether they lie above or below the market clearing price. This process repeats with each market cycle. (Katipamula, Hatley, Hammerstrom, Chassin, \& Pratt, 2006). Figure 2-6 illustrates this example. 


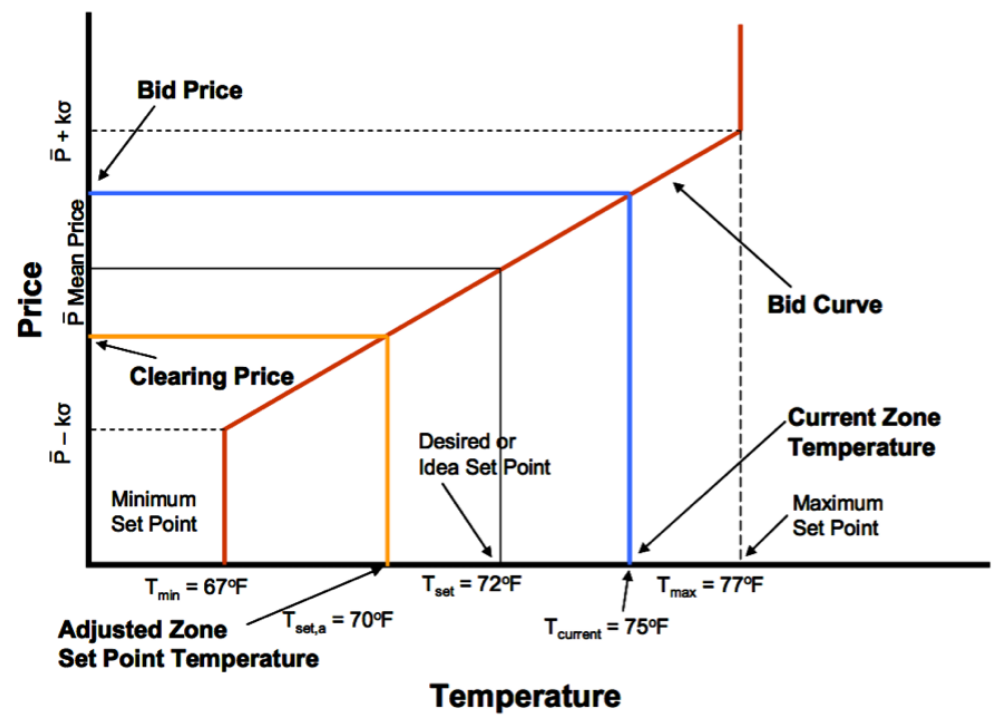

Figure 2-6: Illustration of Biding Strategy of Thermostat in Cooling Mode in Transactive Energy Network (Katipamula, Hatley, Hammerstrom, Chassin, \& Pratt, 2006).

Thermostats operating with transactive control capabilities have been shown to be more active participants in DR than conventional TOU thermostats, yielding improved load shifting, cost savings, demand elasticity, and occupant thermal comfort (Chassin, Stoustrup, Agathoklis, \& Djilali, 2015). Transactive thermostats allow for optimal pre-heating or cooling if predictive algorithms are available (Katipamula, Hatley, Hammerstrom, Chassin, \& Pratt, 2006). Improved total demand elasticity may be achieved by applying these techniques to additional household loads.

Transactive energy network test projects have been investigated through real-world experimentation. The Olympic Peninsula Project evaluated a transactive energy system on a network of 112 residential houses, two industrial buildings, and one commercial building. The project lasted one year from 2006-2007, and gave consumers live in-home energy monitoring capabilities. Thermostats, water heaters, clothes dryers, and commercial HVAC systems were integrated into the transactive energy system. Consumers were allowed to define thermal comfort margins for their thermostats - higher thermal comfort constraints would mean that the thermostats would be less likely to react to pricing incentives from the utility. Through customer engagement, this project was able to achieve reductions in the annual average peak electricity demand by $15 \%$, with peak daily reductions of 50\% (Snowdon \& Ambrosio, 2010). 


\subsection{Experimental Microgrids}

Microgrids have been developed and implemented in the world today, although they are in their early stages of optimization. Their increasing interest is reflected in the development of standards aimed to promote their adoption (IEEE, 2011). Microgrids in the real world typically have one of three control strategies: centralized, autonomous, and agent based.

\subsubsection{Centralized Experimental Microgrids}

A microgrid utilizing centralized control has a complex central controller, and typically lacks flexibility with respect to the addition of new DGs This is currently the most established control method in real world implementation (Lidula \& Rajapakse, 2011).

The DeMoTec microgrid test facility in Germany utilizes a centralized controller. This microgrid network is composed of two battery storage devices, two diesel generators, PV, and wind generation, with a total generation capacity of $200 \mathrm{~kW}$. A supervisory control and data acquisition system (SCADA), a system commonly implemented for process monitoring, is used to control the components of the microgrid. This microgrid also features automatic switching, which allows for its segregation into three separate islanded units (Barnes et al., 2005).

The Bronsbergen Holiday Park in the Netherlands supplies energy to its 208 homes with a gridconnected microgrid. It is operated under the More Microgrids initiative implemented by the European Union. The buildings in this park are outfitted with a total $315 \mathrm{~kW}$ PV modules, with two central battery bank storage units. This microgrid uses a centralized control unit to monitor the active and reactive power flow between the microgrid and utility (Kroposki et al., 2008). Also operating under the More Microgrids initiative is the CESI RICERCA DER test microgrid in Italy. This microgrid also utilizes centralized control with solar, wind, CHP, and diesel generation, along with both flywheel and battery energy storage (Lidula \& Rajapakse, 2011).

The New Energy and Industrial Technology Development Organization (NEDO) supports the development of microgrids in Japan. One example is the Kyoto Eco-Energy Project, a virtual microgrid. It is virtual in that its distributed energy resources and loads are each connected to separate substations, and are only linked by a centralized internet control system (Lidula \& 
Rajapakse, 2011). Another project supported by this initiative is the Aichi Project. This microgrid is comprised of PV, fuel cells, and battery storage. Centralized control is implemented through the battery inverter for load matching (Barnes et al., 2005).

\subsubsection{Autonomous Experimental Microgrids}

Autonomous microgrids allow plug-and-play operability with regards to the addition of new components, such as DGs, without requiring a system redesign. This eliminates the need for a complex centralized controller (Lidula \& Rajapakse, 2011).

The CERTS test bed in Columbus, Ohio, operates without the use of a central controller, and thus supports plug-and-play capabilities. Its power sources operate in a peer-to-peer manner such that losing a component of the system does not interrupt operation (Lidula \& Rajapakse, 2011). This system uses a centralized communication system to communicate set points to the distributed resources, however this component does not operate dynamically with the system (Barnes et al., 2005).

\subsubsection{Agent Based Experimental Microgrids}

The agent based control approach allows for centralized coordination along with distributed local control, combining centralized and decentralized control strategies (Lidula \& Rajapakse, 2011).

Kodiak Island, composed of seven communities totaling 15000 people, is located off the south coast of Alaska. It currently provides its electricity needs through a microgrid utilizing wind and hydro power, along with complementing flywheel and battery energy storage. Nearly the entire 28 MW demand is met by this configuration (ABB, 2014). This isolated microgrid uses ABB's Microgrid Plus System control system - a networked, modular control system responsible for determining the microgrid's most economic mode of operation, while maximizing renewable energy integration (ABB, 2016). This microgrid solution provider, ABB Group, has also implemented microgrids in other remote communities such as Faial Island, Azores, and Coral Bay, Australia (ABB, 2016). 
Am Steinweg, Germany, is home to a residential microgrid test bed. This microgrid is comprised of 101 apartments, a CHP, $35 \mathrm{~kW}$ of PV, and a battery bank. This microgrid does not operate entirely independently from the grid, but is reported to have this capability at times of excess renewable generation. The microgrid utilizes a Power Quality Management System (PoMS) consisting of a central unit with decentralized communication units. Controllable devices communicate with the central unit through the decentralized units using TCP/IP communication protocol (Erge et al., 2005).

The laboratory-scale microgrid system at the National Technical University of Athens (NTUA), Greece, utilizes a transactional energy control structure, with four agent types. The Production Unit is responsible for control of the battery. The Consumption Unit is responsible for the controllable loads in the microgrid, making load predictions every 15 minutes, and communicating those needs to the production units. The Power System communicates the buying and selling price of the main utility grid, but does not participate in market activity. The Microgrid Central Controller coordinates tasks between the different agents and records information on the transactions that occurred. The control systems for this microgrid were developed using WinCC and LabVIEW software (Barnes et al., 2005).

\subsection{Optimal EMS Control Techniques}

A variety of optimization and solution-based approaches are being actively investigated throughout literature. These optimization techniques can use a wide variety of objective functions, although they typically aim to minimize operating costs associated the utility grid, or fuel consumption. However, they may also be derived to minimize other parameters such as carbon emissions or energy storage utilization (Khan, Naeem, Iqbal, Qaisar, \& Anpalagan, 2016). This section presents some of the more prominent techniques for optimal EMS and microgrid control.

\subsubsection{Mixed Integer Linear Programming}

Mixed-integer linear programming (MILP) is an optimization technique that handles problems involving both discrete and continuous variables. Using this programming technique, a cost function can be minimized to achieve optimal power flow within a microgrid. The cost function is 
subject to the constraints of the system, such as renewable generation limits and energy storage capacity. This is a well-established method of programmatic optimization.

MILP is an effective tool that has been successfully used to determine optimal microgrid operation for systems utilizing wind, solar PV, fuel cells, and battery storage (Morais, Kádár, Faria, Vale, \& Khodr, 2010). It has been used to determine optimal microgrid configuration with respect to capital and operational costs (Palma-Behnke et al., 2013). This technique can be used for optimization purposed in conjunction with additional energy management strategies. One example involves the use of MILP to determine optimal microgrid configuration in conjunction with DR strategies in order to minimize operational costs (Alharbi \& Bhattacharya, 2013).

\subsubsection{Mixed Integer Nonlinear Programming}

As many physical phenomena in nature are not linear, mixed integer nonlinear programming (MINLP) techniques also have applications for microgrid operational optimization. This type of system is more complicated, but does not involve the approximations made by a linear system. A nonlinear programming method, in conjunction with fuzzy logic for pricing schemes, has been shown to achieve revenue for a microgrid under an air pollution reduction policy (Manjili, Rajaee, Jamshidi, \& Kelley, 2012). Experimental microgrid test-bed validation has shown that MINLP can achieve faster processing speeds, along with $15 \%$ cost of energy reductions, when compared to a conventional EMS (Marzband, Sumper, Domínguez-García, \& Gumara-Ferret, 2013).

\subsubsection{Stochastic Programming}

Stochastic programming is a method of optimization that accounts for uncertainties in a system. This programming technique presents a potential solution to account for the intermittency of renewables in a microgrid. Monte Carlo simulations have been used to optimize microgrid operation by accounting for renewables, component availability, and failure rates, investigating the operation of both an isolated microgrid, as well as a set of three interconnected microgrids (Giacomoni, Goldsmith, Amin, \& Wollenberg, 2012). Researchers have used stochastic optimization models in order to develop an energy and reserve scheduling program for a microgrid with active consumer demand response initiatives. This model has shown that higher consumer involvement leads to decreased microgrid operating costs, and decreased diesel generator usage 
(Zakariazadeh, Jadid, \& Siano, 2014). Comparisons of stochastic and deterministic approaches in microgrids shows that the stochastic model better accounts for intermittency in renewable generation, and provides better energy scheduling to minimize power losses ( $\mathrm{Su}$, Wang, \& Roh, 2014).

\subsubsection{Heuristic Programming}

Heuristic programming is an artificial intelligence (AI) technique used to solve complex problems. These approaches use "common sense" based on past experience and measurements to select a desired action. Heuristic approaches have been developed for smart grid consumption scheduling using non-cooperative game theory ( $\mathrm{Ma}, \mathrm{Hu}, \&$ Spanos, 2013). A heuristic approach to microgrid automation has been developed in order to minimize cost (Khederzadeh, 2013). Heuristic approaches have been used to determine energy consumption trends, such that a microgrid may relay household consumption forecasts to the utility grid (Hino, Shen, Murata, Wakao, \& Hayashi, 2013).

\subsubsection{Artificial Neural Networks}

Electrical load forecasting will play an integral role in optimizing building energy management. Many AI techniques such as genetic algorithms (GAs) and artificial neural networks (ANNs) have shown promise in microgrid and load forecasting applications, with ANNs receiving significant attention (Bansal \& Pandey, 2005).

ANNs can be a useful tool in microgrid load and generation forecasting. They represent an adaptive forecasting approach that can handle systems with a large number of inputs. In an ANN, weights are applied to the system inputs through "neurons", such that a desired output may be achieved. An ANN requires calibration for the system it is applied to, a process known as training. This technique can be used to optimize microgrid predictions, improving their reliability. Figure 2-7 shows a general example of an ANN that may be used for solar energy predictions based on certain inputs. 


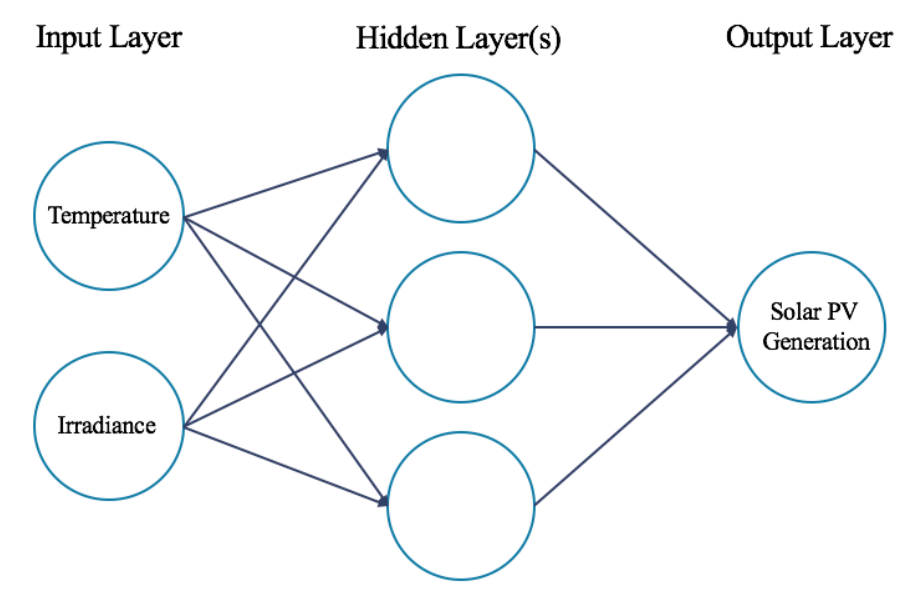

Figure 2-7: An example of an ANN used for solar PV generation predictions

ANN can be used for day, season, or year-ahead load forecasting for microgrid operation. Dayahead forecasting proves useful for EMS transactions between microgrids, or a microgrid and the utility. Seasonal and yearly forecasting may aid in maintenance planning (Reddy, Kumar, Kumar, \& Raju, 2012). Recurrent neural networks (RNN) allow for bidirectional data flow, and have also been applied to microgrid operation. A RNN for microgrid operation has been shown to optimize power supply, by minimizing energy purchased from the utility grid and maximizing renewable energy generation (Gamez Urias, Sanchez, \& Ricalde, 2015). This optimized forecasting technique can reduce the risk of unforeseen energy shortages, and greatly aid in microgrid planning and scheduling.

\subsubsection{Genetic Algorithms}

GA optimizes a problem utilizing the principles of genetics and natural selection; it searches a large population for individual optimal solutions, typically minimized cost. This is a powerful optimization technique, particularly for systems containing a large number of variables. It can be used in conjunction with other AI techniques.

GA initiates by defining a random set of binary variable strings, known as chromosomes, representing potential optimal solutions. Each chromosome is a potential solution in the search space with an associated cost function. In GA, the chromosome elements are represented as bits with binary encodings -0 or 1 . A fitness function (which can be the cost minimization function) is used to assign each chromosome in the population a score. After the fitness of all the candidate 
solutions has been determined, chromosomes are randomly selected (usually weighted by their ranking) to perform crossover, and a new population is created. The process repeats and continues to generate offspring with greater and greater fitness until convergence on a solution (Mitchell, 1999). Figure 2-8 illustrates a general crossover process. Typically, a decoder function is used throughout this process to translate the binary chromosomes into real-world values.

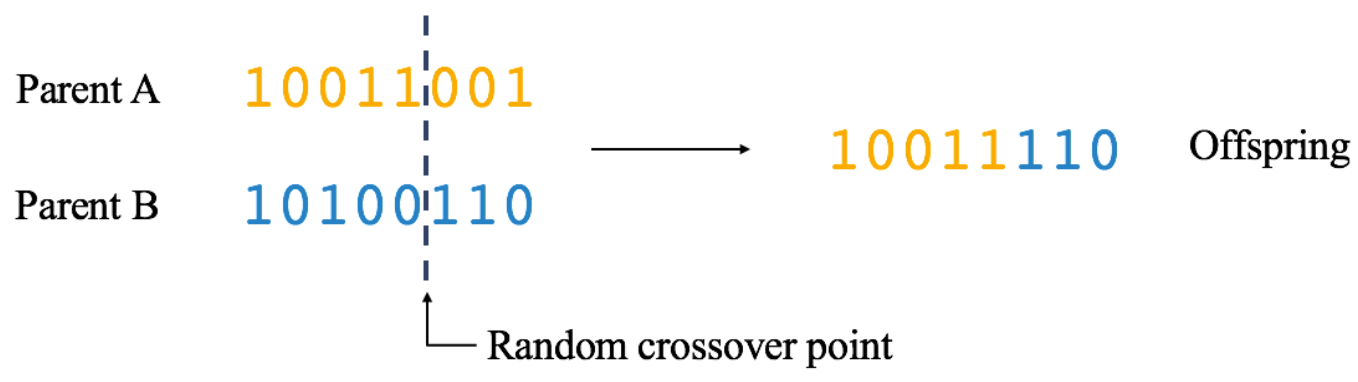

Figure 2-8: Example of GA crossover

With respect to microgrid operation, the fitness function will almost always be a cost function. GA has been used for optimization of systems under a carbon trading program (Sadegheih, 2011). This tool is very powerful for large systems with many variables that would typically have long computation times. It could therefore prove useful for large-scale microgrid applications. Simulations on a microgrid with a network of capacitors as energy storage show that GA effectively and quickly determine optimal solutions (Gholami, Shahabi, \& Haghifam, 2011). GA has been utilized to determine PI controller parameters for stable operation of a microgrid with wind power and a diesel generator (Nandar, 2013). It has also been used to optimize battery energy storage in microgrids (Corso, Di Silvestre, Giuseppe, Riva, \& Zizzo, 2010). GA effectively converges on multi-objective operational solutions for microgrids, optimizing operating costs, battery lifetime, and controllable load management (Khan, Naeem, Iqbal, Qaisar, \& Anpalagan, 2016).

\subsubsection{Model Predictive Control}

Model Predictive Control (MPC) is a robust control technique utilized across many different industries. MPC uses dynamic models to predict the future state of the system. Predictions are optimized such that the optimal control option can then be applied (Rawlings \& Mayne, 2016). 
Historical data may also be used to aid in state estimations. Future system states are dynamically estimated over a prediction horizon and a control input is chosen to minimize an objective (cost) function (Allgöwer, Findeisen, \& Nagy, 2004). Figure 2-9 illustrates the concept of MPC.

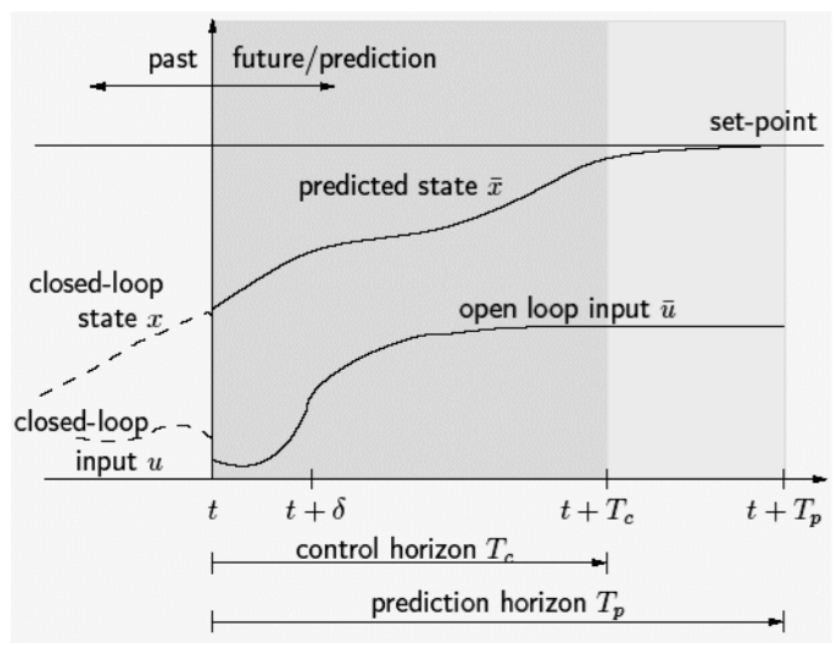

Figure 2-9: Model Predictive Control Concept

MPC algorithms will play a large role in future smart and microgrid applications (Minchala-Avila, Garza-Castañón, Vargas-Martínez, \& Zhang, 2015). These algorithms prove to be particularly helpful in predicting intermittent renewable energy production. Supervisory MPC has been applied to integrated solar/wind microgrid models. One such model showed successful grid integration in conjunction with control of a water desalination system, with the ability to smooth water production using predictive algorithms (Qi, Liu, \& Christofides, 2011). MPC algorithms have been shown to contribute to increased controller robustness for solar PV/wind systems under varying environmental conditions (Qi, Liu, Chen, \& Christofides, 2011). Utilizing MPC techniques can also improve system resilience by helping to mitigate potential battery or generator failures by accounting for component health in state predictions (Ionela \& Zio, 2014).

Optimal load switching using MPC has been shown to be successful in solar/wind microgrid systems with hybrid hydrogen/capacitor/battery energy storage. The addition of hydrogen storage to the system mitigates high battery stress current scenarios (Garcia-Torres, Valverde, \& Bordons, 2016). MPC allows optimal battery/fuel cell utilization ensuring equipment health is maintained, increasing microgrid system lifetime (Valverde, Bordons, \& Rosa, 2012). Experimental validation 
has demonstrated that MPC strategies promoting constant fuel cell current output, allowing the battery to smooth PV and load variability leads to improved performance, with the potential for system size reduction (Bifaretti et al., 2015).

Due to system nonlinearities, applying MPC to complex microgrids can potentially lead to large computation times. However, it has been shown that this may be addressed by breaking down the overall energy management problem into unit commitment and optimal power flow components. This yields separate linear and nonlinear models, which produces computation times within practical real-world application limits (Olivares, Cañizares, \& Kazerani, 2014)

MPC algorithms can achieve $25.3 \%$ and $28.5 \%$ energy and cost saving potentials respectively (Ma, Qin, Salsbury, \& Xu, 2012). These savings were realized through automatic pre-cooling, resulting in a load shifting effect. Houses also have the potential for thermal storage, which can then manage electricity demand for heating or cooling purposes. MPC algorithms utilizing thermal energy storage have successfully shown improvements in building heating performance (Candanedo \& Athienitis, 2011).

MPC strategies have been applied to an experimental microgrid system in Athens, Greece. This microgrid consists of solar PV, distributed generators, and battery storage units. MPC-MILP strategies produced $28.5 \%$ energy savings relative to baseline with a prediction horizon of 24 hours, which were increased to $34.7 \%$ savings using an extended horizon of 72 hours (Parisio, Rikos, \& Glielmo, 2014). 


\subsubsection{Literature Review Conclusions}

Clearly, research has already explored a vast variety of different EMS and microgrid control systems of varying complexity. However, much of the literature investigates the use of a single type of control system in a single application. This means that EMSs cannot be directly compared, because there is a shortage of data available with respect to the operation of different types of EMSs operating under identical conditions. This research aims to directly compare different EMS control strategies by applying them to the exact same system.

Of course, not all EMS control strategies can be investigated in a reasonable amount of time, and only a select few EMS models have been selected for investigation. The EMS models developed for this work are described in detail in Chapter 3. They have been designed in part with influence from various components of this literature review, and otherwise are based on the author's discretion. 


\section{Methodology}

This work has investigated a variety of different EMS options for residential buildings. Various control and logic options were explored using both simulation tools and experimental trials. The primary focus of the EMSs investigated is battery bank management and control for residential buildings.

Simulations in MATLAB, using a variety of different software tools, were utilized to investigate the impact of various cases. This section describes how the various logic behind decisions made by the different EMSs, while Chapter 4 describes the simulation methods in detail. Simulation work was necessary in order to investigate the long-term impact of different EMSs.

Experimental work was conducted in order to validate the application of different EMSs. Since experimental trials could not be conducted over a period of a year, their results represent the validation of different EMS options - or their proof-of-concept.

The energy management techniques investigated in this thesis are broken down into four main types: baseline, deterministic, optimal, and adaptive. Baseline simulations were run without control logic, in order to quantify the expected energy consumption and cost for different scenarios involving loads, with and without renewables. The latter three cases involve the addition of a battery bank, with control logic primarily focused on managing charging and discharging.

For the purposes of this work, a deterministic EMS is defined as one with predefined operations that do not change throughout the year. This means that decisions, with regards to battery or load control, are all defined at the beginning of the year/simulation, and do not change as time passes. An example would be a programmable set-back thermostat, allowing greater deviation from its set point for the same six hours a day (when occupants are at work/asleep) for an entire year.

An adaptive EMS is one whose control and logic outputs are dependent on the conditions of the building at any given point in time. While they do have predefined rules, the outcome of these rules will always be unknown until the point in time at which the system is evaluated. An example of this type of control would be predictive or transactive control. 
The optimal EMS is investigated mainly for comparison purposes, and is likely not achievable in reality. However, the optimal EMS is used in some cases as a training platform for the adaptive EMSs. This optimized case analyzes the building's load and generation profile after-the-fact, and determines what the optimal battery decision (charging or discharging) would have been. By knowing both the baseline and optimal savings the EMSs investigated can be ranked. It can also give insight as to whether adding "smarts" to an EMS is worthwhile - if a simpler EMS is "close enough" to the optimal case, is increasing the intelligence of the control system worth it?

\subsection{Baseline Scenarios}

Baseline simulations defined the electricity consumption and operating costs associated with the normal operation of residential buildings. All buildings investigated in this work operate using allelectric appliances - including those used for space heating and water heating. This simplification meant that energy conversion factors between natural gas and electricity could be ignored. It also simplified energy and associated cost and GHG emission calculations. The cost of electricity, and calculation of the grid emission factor, are described below.

\subsubsection{Ontario Electricity Prices}

Residential electricity charges in Ontario, Canada, are applied on a time-of-use (TOU) basis. This passive form of DR is designed to incentivize consumers to use less electricity during peak periods of the day. The hourly schedule for this pricing scheme, as of May $1^{\text {st }}, 2017$, is shown in Figure 3-1.

\begin{tabular}{|c|c|c|c|c|c|c|c|c|c|c|c|c|c|c|c|c|c|c|c|c|c|c|c|c|}
\hline Hour & 0 & 1 & 2 & 3 & 4 & 5 & 6 & 7 & 8 & 9 & 10 & 11 & 12 & 13 & 14 & 15 & 16 & 17 & 18 & 19 & 20 & 21 & 22 & 23 \\
\hline $\begin{array}{c}\text { Winter } \\
\text { November 1 - April 30 }\end{array}$ & & & & & & & & & & & & & & & & & & & & & & & & \\
\hline $\begin{array}{c}\text { Summer } \\
\text { May 1 - October 31 }\end{array}$ & & & & & & & & & & & & & & & & & & & & & & & & \\
\hline
\end{tabular}

$\begin{array}{ccc}\text { Off-peak } & \text { Mid-peak } & \text { On-peak } \\ 7.7 \phi / \mathrm{kWh} & 11.3 \phi / \mathrm{kWh} & 15.7 \phi / \mathrm{kWh} \\ (11.2 \phi / \mathrm{kWh}) & (14.8 \phi / \mathrm{kWh}) & (19.2 \phi / \mathrm{kWh})\end{array}$

Figure 3-1: Ontario TOU pricing schedule including electricity prices and total cost per $\mathrm{kWh}$ in brackets $(O E B, 2017)$ 
In addition to the advertised price of electricity, the local distribution companies (LDCs) also charge delivery and regulatory fees for electricity. Some LDCs also charge an additional transmission fee. Both of these charges have variable $(\phi / \mathrm{kWh})$ and fixed $(\$ /$ month) components. The breakdown of these costs for the Toronto Hydro jurisdiction are shown in Table 3-1 and Table $3-2$. In addition to these costs, a $5 \%$ tax is applied to the final billed amount, accounting for an $8 \%$ provincial tax rebate recently put into effect.

Table 3-1: Toronto Hydro variable electricity prices as of May 1 $1^{\text {st }}, 2017$ (OEB, 2017)

\begin{tabular}{|l|c|c|c|c|}
\hline TOU period & $\begin{array}{c}\text { Advertised } \\
\text { electricity cost } \\
(\boldsymbol{d} / \mathbf{k W h})\end{array}$ & $\begin{array}{c}\text { Variable } \\
\text { delivery cost } \\
(\boldsymbol{c} / \mathbf{k W h})\end{array}$ & $\begin{array}{c}\text { Variable } \\
\text { regulatory cost } \\
(\boldsymbol{d} / \mathbf{k W h})\end{array}$ & $\begin{array}{c}\text { Total variable } \\
\mathbf{c o s t}(\boldsymbol{c} / \mathbf{k W h})\end{array}$ \\
\cline { 1 - 2 } Off-peak & 7.7 & \multirow{2}{*}{2.95} & 0.59 & $\mathbf{1 1 . 2}$ \\
\cline { 1 - 2 } Mid-peak & 11.3 & & $\mathbf{1 4 . 8}$ \\
\cline { 1 - 2 } On-peak & 15.7 & & $\mathbf{1 9 . 2}$ \\
\hline
\end{tabular}

Table 3-2: Toronto Hydro fixed electricity prices as of May $1^{\text {st }}, 2017$ (OEB, 2017)

\begin{tabular}{|l|c|}
\hline Charge type & Cost (\$/month) \\
\hline Fixed delivery cost & 28.63 \\
\hline Fixed regulatory cost & 0.26 \\
\hline Total fixed cost & $\mathbf{2 8 . 8 9}$ \\
\hline
\end{tabular}

The costs shown in Table 3-1 and Table 3-2 are used for all costs calculations with respect to energy drawn from the grid. Neglecting maintenance costs, it is assumed that there is no operating cost associated with the operation of DGs and battery banks. When a finding or result references the "variable cost" of operation, it has used only the values in Table 3-1, and neglects all fixed costs. This has been done for some cases that do not represent the entire electrical load of the house.

\subsubsection{Ontario Grid Emission Factor}

In 2014, Ontario phased out its last coal-fired electric generation. Prior to the coal phase-out, the emission factor of the Ontario electricity grid was in the range of $189-221 \mathrm{gCO}_{2} \mathrm{eq} / \mathrm{kWh}$, in the years $2004-2006$ (Gordon \& Fung, 2011). Currently, the only remaining fossil fuel used for electricity generation in the province is natural gas, making for much cleaner electricity 
production; the life-cycle emission factor of natural gas is less than half of that of coal (Moomaw et al., 2011). Figure 3-2 shows the fuel mix of the Ontario electricity grid for the year 2015 based on total kWh generated by each generation type currently employed.

At any given time of day, the actual fuel mix of the Ontario grid varies, meaning that greenhouse gas (GHG) emissions associated with electricity use change hour-to-hour. Generation supply data for the year 2015 was provided by the Independent Electricity System Operator (IESO) in order to analyse the GHG emission changes on an hourly basis. Using hourly GHG emission factors, the effect of load shifting on associated GHG emissions could be determined. Table 3-3 displays the emission factors used to determine the emissions associated with electricity. These emission factors are based on lifecycle analysis of each generation source, and should therefore represent accurate and conservative values.

\section{Ontario Electricity Generation by Fuel Type}

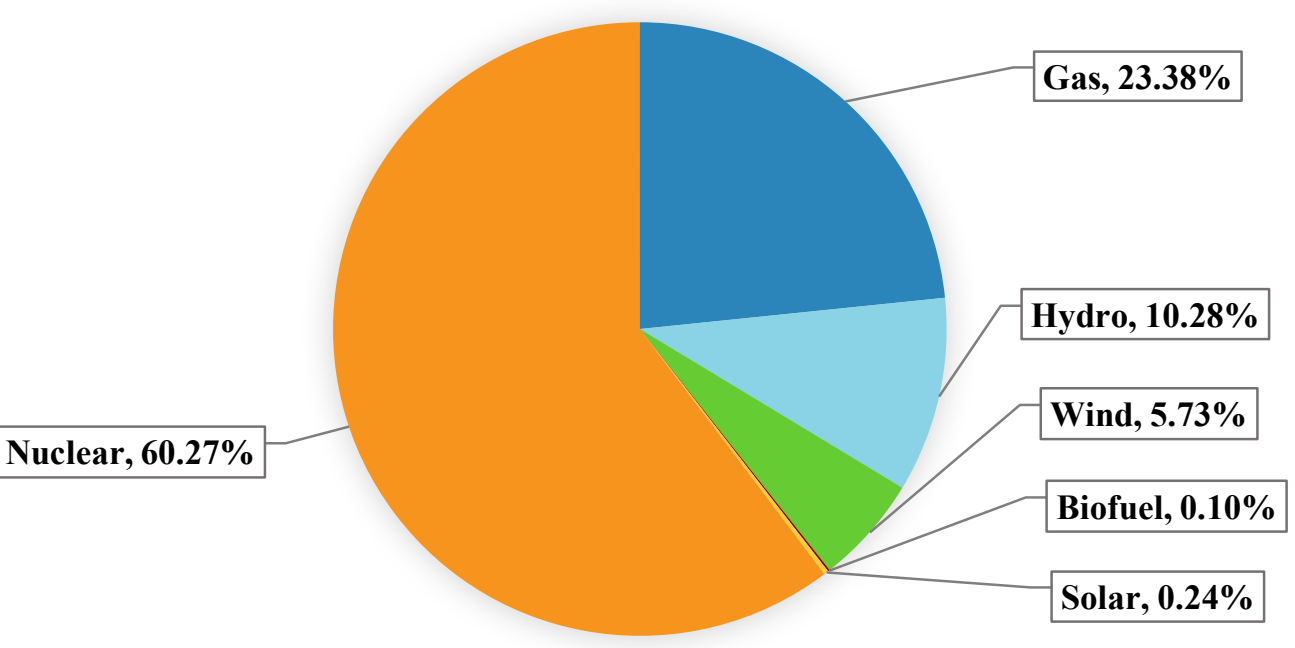

Figure 3-2: Ontario Electricity Grid Generation by Fuel Type (IESO, 2016)

Using generation data from the IESO for the Ontario electricity grid, the emission factor of the grid $\left(\mathrm{gCO}_{2} \mathrm{eq} / \mathrm{kWh}\right)$ could be determined using the values in Table 3-3. Hourly emission factor averages based on the 2015 dataset are presented in Figure 3-3. The annual average emission factor for this year was $58.1 \mathrm{gCO}_{2} \mathrm{eq} / \mathrm{kWh}$. The exact values in Figure 3-3 were not used in calculations, 
rather, they illustrate which hours of the day have the highest associated emission factors. Hourly emission factor data was used for all calculations.

Table 3-3. Lifecycle emission factors of electricity generation sources (Moomaw et al., 2011)

\begin{tabular}{|l|c|}
\hline $\begin{array}{c}\text { Generation } \\
\text { Source }\end{array}$ & $\begin{array}{c}\text { Lifecycle GHG emission } \\
\text { factors }\left(\mathbf{g ~ C O}_{\mathbf{2}} \text { eq./kWh) }\right.\end{array}$ \\
\hline Coal & 1001 \\
\hline Natural Gas & 469 \\
\hline Nuclear & 16 \\
\hline Hydro & 4 \\
\hline Wind & 12 \\
\hline Biofuel & 18 \\
\hline Solar & 46 \\
\hline
\end{tabular}

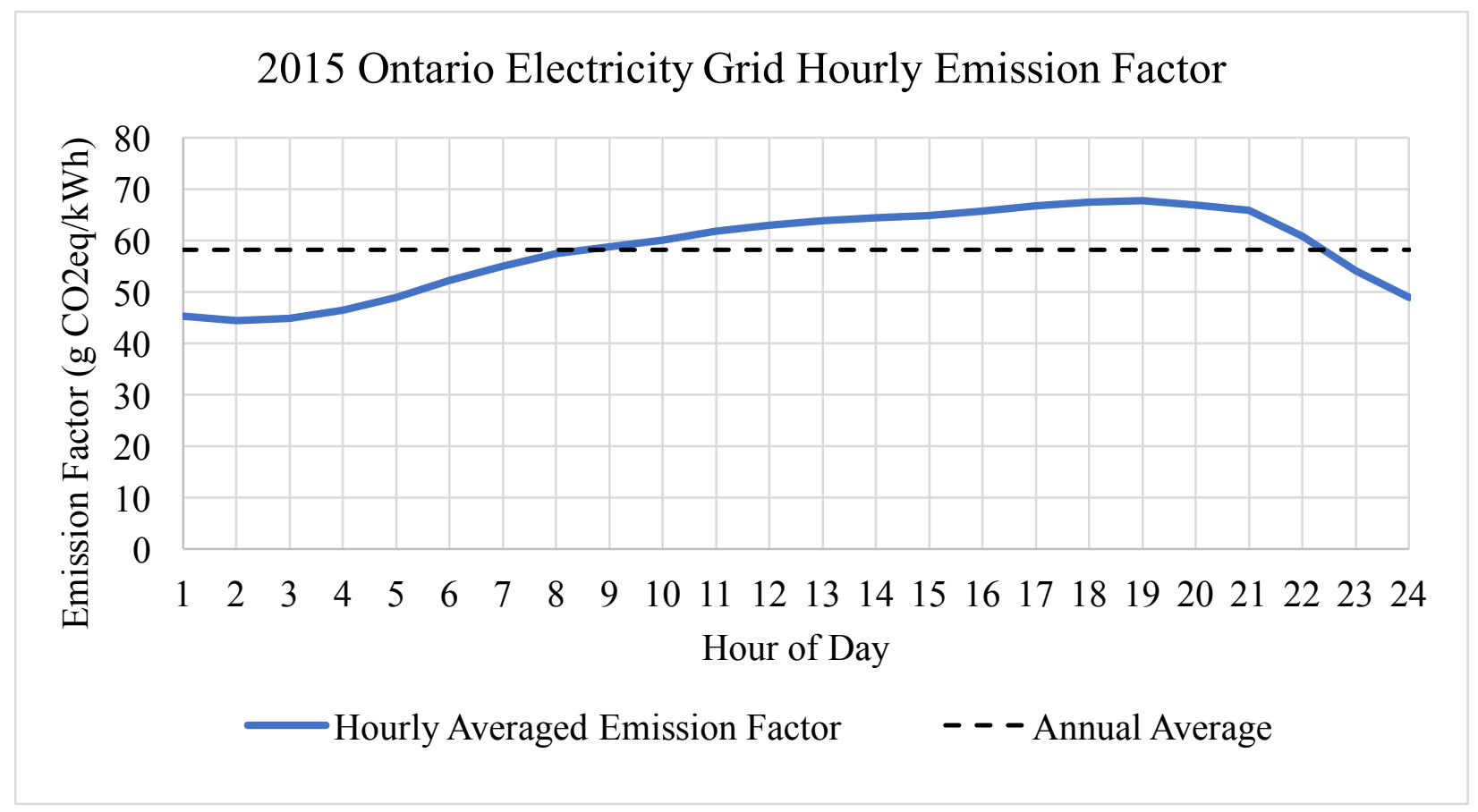

Figure 3-3: Hourly averaged emission factors based on 2015 IESO generation data

Using the Ontario electricity grid pricing scheme and emission factor profile, the annual operational costs and emissions associated with different control options can be quantified. Baseline scenarios involved no control of loads or DGs, and never had battery banks. In all cases, it was assumed that DGs operated on a zero-feedback basis. This means that their instantaneous power generation was used to offset any load at that moment in time. If there was an excess in 
renewable generation, it was assumed that the device's inverter either throttled the generation to match the load, or exported the excess power to the grid without price incentives. Neither feed-intarrif (FIT or microFIT), nor net metering contracts were investigated, so that all financial savings found could be attributed to control actions alone.

\subsection{Deterministic Energy Management System Description}

The deterministic EMSs investigated in this work are another form of baseline. They represent in the author's opinion - the simplest form of battery control. Two types of deterministic battery control were analyzed, both of which involved load shifting based on the Ontario TOU pricing schedule.

\subsubsection{Basic Load Shifting}

The most basic form of load shifting involves using the battery bank to offset a building's load on the grid during peak TOU hours. The battery bank was used to perform load shifting by matching the load of the building between the hours of 7:00 - 19:00, shifting all mid- and on-peak consumption to off peak times of day. After 19:00, the battery bank was allowed to recharge from the grid using the cheapest electricity available. This practice could be performed both with and without renewables.

The addition of DGs to the basic load shifting case represents the absolute simplest configuration a homeowner could implement when combining renewable generation and battery energy storage - a configuration in which those two components are not connected at all. In this case, the electrical load would first be offset by renewable generation, and any remaining load on the grid would be matched by the battery bank. The battery bank would then be recharged at 19:00, and any excess renewable generation at off-peak times would be used to offset battery charging or household load.

While this may not be the most realistic configuration of components, it is still of interest to investigate any savings that may be associated with this setup, as it represents the absolute least one could do to integrate these two types of systems. An example of where this might occur is if a 
homeowner had an existing grid-tied renewable energy system, and later added a battery bank on a different branch of their home circuit without the capability of dynamic charging.

\subsubsection{Load Shifting with Excess Charging}

The second deterministic EMS represents what is potentially a more common case - the integration of renewables with battery energy storage such that excess renewable energy is stored in the battery bank. This method is similar to the load shifting with zero-feedback option, however, in this case excess renewable electricity generation is used to charge the battery bank, instead of simply being throttled to match the load. This method of system integration ensures that all of the renewable electricity generation is utilized. In addition to using excess electricity generation to recharge the battery bank, load shifting between 7:00 and 19:00 is again implemented. This practice still involves recharging the battery bank overnight, however, the amount of energy required to recharge the battery will, in theory, be reduced.

\subsection{Optimized Battery Management System}

The problem of optimal battery control has an immense solution space; the problem must be simplified in order to efficiently solve this issue. A custom MATLAB genetic algorithm code was developed to perform the optimization procedure.

In order to simplify the problem, it was assumed that the battery could operate in one of two states: discharge enabled and charge enabled. Breaking down the problem into these two states neglects the option of doing nothing. Initially, a triple-base chromosome GA model was applied to the problem. As opposed to conventional chromosomes, formed of a series of 0's and 1's, a model was used with a third option, represented by the number 2 . This drastically increased the solution space size, along with the complexity of the code. However, after various test trials, it was determined that the third option (2) was rarely selected, and that the triple-base GA model only achieved a $0.1 \%$ improvement in energy savings over its traditional, double-base counter part. It was therefore deemed appropriate to neglect the third option of doing nothing. 
When operating in discharge enabled mode, the battery bank will act as it does during the day time when using the load shifting with excess charging protocol - discharging to meet the net load if the load exceeds the generation, or charging if the generation exceeds the load. During charging enabled mode, the battery will charge using the grid and whatever renewable generation is being produced at that time.

At this point, the problem has been broken down into a matter of determining whether to enable charging (state 0 ) or discharging (state 1) at every given time step throughout the simulation timespan. In order to solve this issue in a reasonable amount of time, the solution space was broken down into hourly time segments over the course of the year. The problem was tackled day-by-day, with a solution space size of $2^{24}$ for every day of operation. A custom genetic algorithm (GA) code was developed to determine the optimal solution for the battery management problem.

The use of GA fits the problem well - having reduced potential solutions, to 1's and 0's foregoes the need for a chromosome decoder, and allows potential chromosomes to be easily evaluated and operated on. A candidate solution to the optimization problem is referred to as a chromosome, which is a series of 24 bits (1's and 0's). Figure 3-4 shows an example of a random chromosome's effect on the electrical load of the house over the course of a day. The process of the custom GA code is as follows:

1. Initialize random population of 50 chromosomes - approximately double chromosome length (Mitchell, 1999),

2. Evaluate and rank the fitness of the population based on minimizing electricity cost,

3. Perform elitist selection - save top candidate(s),

4. Select two parent chromosomes based on ranked probability:

a. if they are equal, generate a child chromosome at random,

b. otherwise preform random crossover,

5. Add child chromosome to population,

6. Repeat steps 4 and 5 until the population size limit has been reached,

7. Perform random mutations on all but the top solution,

8. Return to step 2, unless the maximum number of iterations has been met, or convergence has been reached,

9. Output solution. 
This process follows the framework standard GA procedure (Mitchell, 1999). Specific parameters related to the GA and selection process are summarized in Table 3-4. These parameters were chosen such that they could locate the global optimum in a reasonable amount of time. The resulting GA can reach convergence in less than one second, requiring an average of 30 iterations.

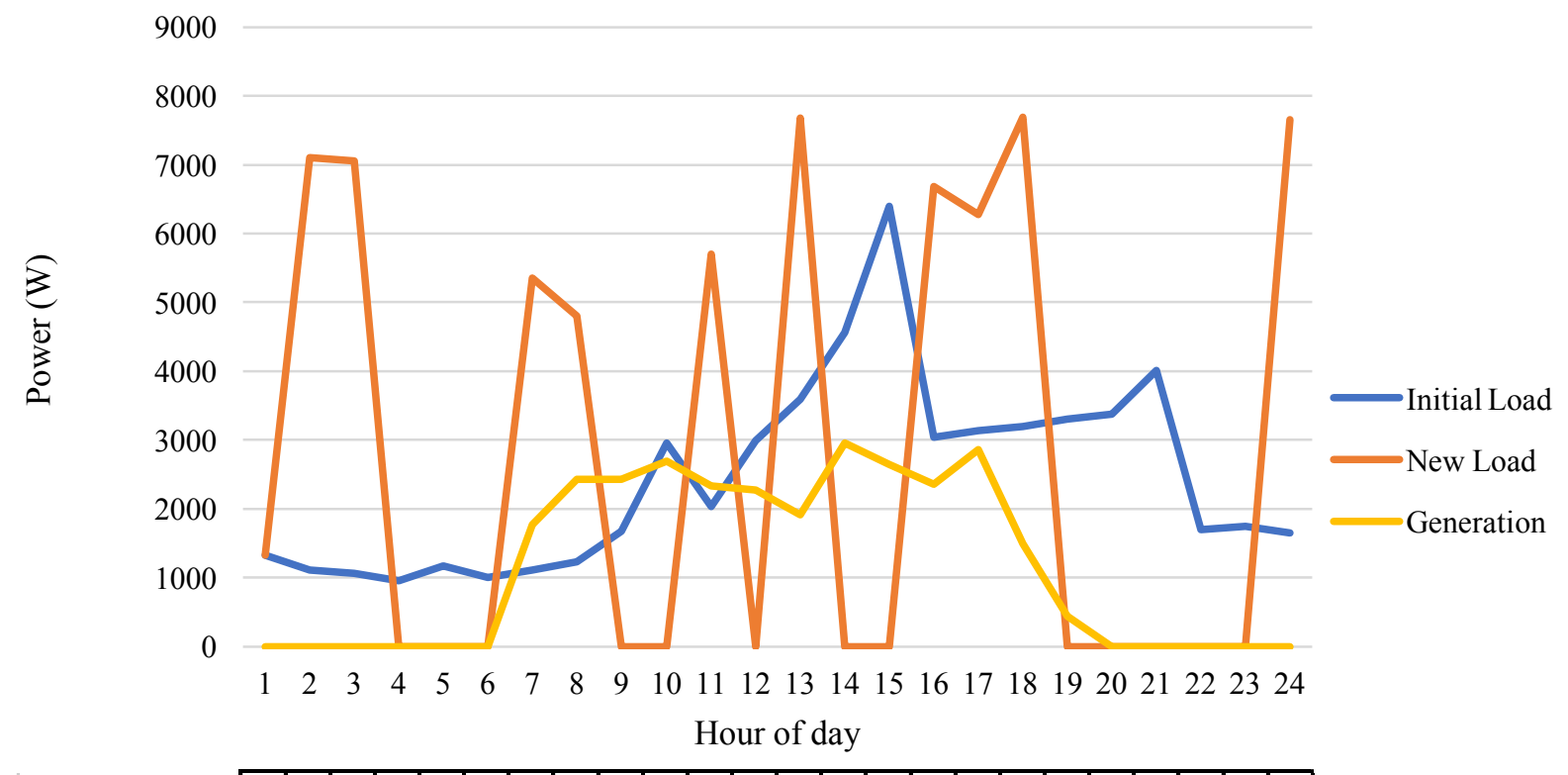

Chromosome \begin{tabular}{|l|l|l|l|l|l|l|l|l|l|l|l|l|l|l|l|l|l|l|l|l|l|l|}
1 & 0 & 0 & 1 & 1 & 1 & 0 & 0 & 1 & 1 & 0 & 1 & 0 & 1 & 1 & 0 & 0 & 0 & 1 & 1 & 1 & 1 & 1 \\
\hline
\end{tabular}

Figure 3-4: Example of the resulting load of a chromosome evaluation

Table 3-4: Genetic algorithm parameters

\begin{tabular}{|l|c|c|}
\hline \multicolumn{1}{|c|}{ Parameter } & Value & Reason for selection \\
\hline Bit length & 24 & Equal to hours in a day \\
\hline Population size & 50 (Mitchell, 1999) & $\begin{array}{c}\text { Recommended approximately } \\
\text { double the bit length }\end{array}$ \\
\hline Elitism & Only top solution survives & $\begin{array}{c}\text { Greater elitism numbers led } \\
\text { to premature convergence }\end{array}$ \\
\hline Crossover probability & $60 \%$ (Mitchell, 1999) & $\begin{array}{c}\text { Trial and error, within } \\
\text { reasonable range }\end{array}$ \\
\hline Mutation probability & $1 \%$ (Mitchell, 1999) & $\begin{array}{c}\text { Trial and error, within } \\
\text { reasonable range }\end{array}$ \\
\hline
\end{tabular}

To determine the optimal annual battery management option, the GA was run on daily consumption/generation load profiles for an entire year. The output was a solution, 8760 bits in 
length, dictating what state to operate the battery bank for every hour of the year to achieve the lowest electricity cost.

\subsection{Adaptive Energy Management System Description}

The adaptive EMSs investigated in this work involve some sort of intelligence and/or inter-device communication. Three types of adaptive EMSs have been investigated. The first system uses machine learning to make decisions based on historical data. The second uses weather forecasts to predict for future generation and consumption. The final adaptive EMS is a transactional energy system that involves load and battery bank control.

\subsubsection{Machine Learning Battery Management System}

The GA-optimized EMS was used to develop a classifier machine learning system (MLS) that can be trained using historical data. It requires no knowledge of the future system states, and can make decisions based on historical data training and current system conditions.

As inputs, the MLS accepts hourly averaged electrical loads, renewable generation, TOU electricity prices, and hour of the day. It was designed to output the same 0 or 1 battery state identifiers as the GA optimization process produced. The training process for the MLS in the realworld would follow the following steps:

1. Collect historical load and renewable generation information, averaged by hour, to reduce data storage requirements,

2. At defined intervals (midnight once per week or month) run the GA optimizer on historical hourly averaged load and generation profiles,

3. Train the MLS using hourly averaged profiles, TOU electricity prices, hour of the day, and the output of the GA optimization program.

Developed using the MATLAB Classification Learner Toolbox, the MLS used the medium knearest neighbours $(\mathrm{KNN})$ classification training protocol. This algorithm classifies the input objects based on their proximity to their nearest neighbours $(\mathrm{k}=10$ neighbours in the case of 
medium KNN). While the medium KNN method produced optimal training results, all training protocols available in the toolbox resulted in classification accuracies above 93\%. Table 3-5 shows the confusion matrix resulting from the training of the MLS on one month's worth of data, while Figure 3-5 displays the receiver operating characteristic (ROC) curve for the same data.

Table 3-5: Confusion matrix for battery control classifier using four weeks of data and medium

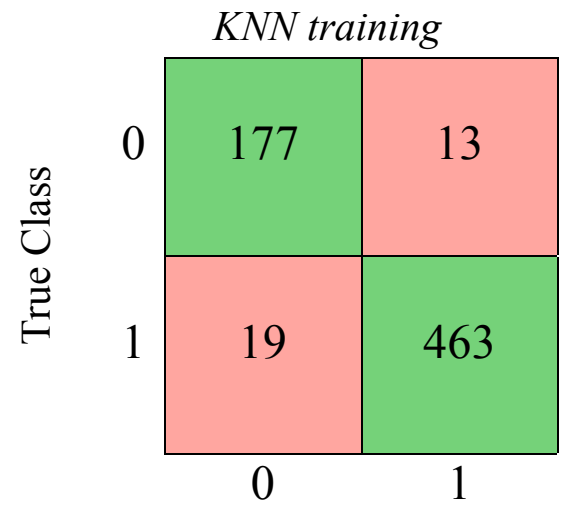

Predicted Class

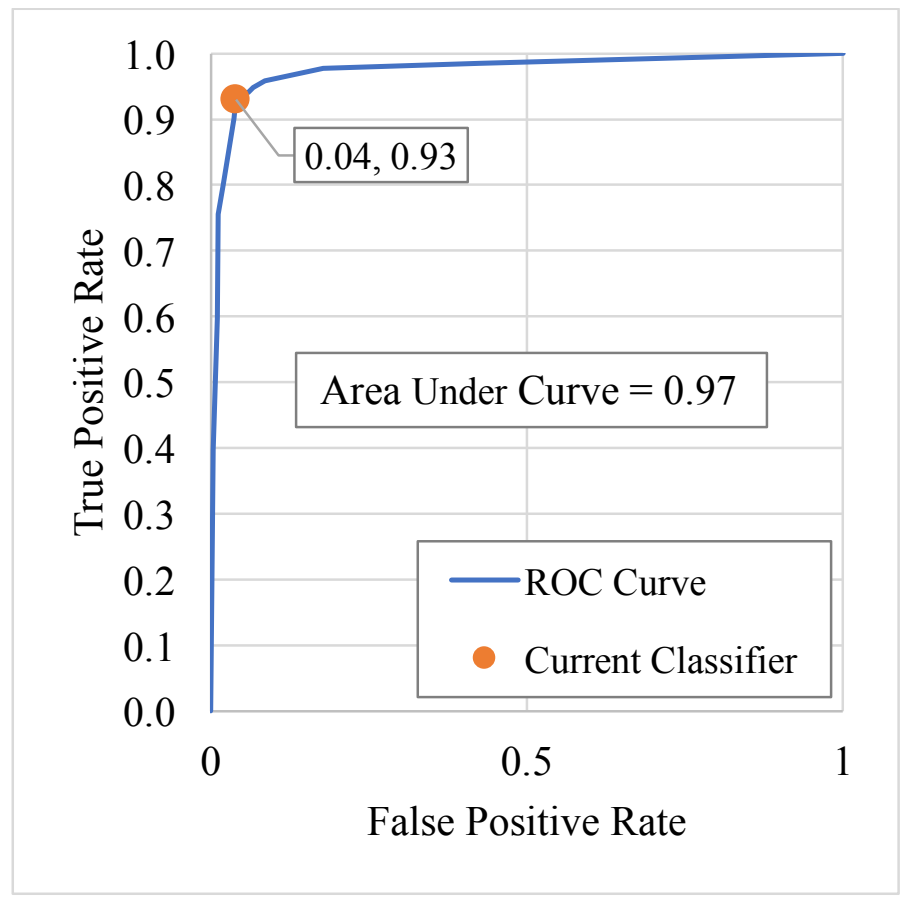

Figure 3-5: ROC curve for classifier training using one month of data under medium KNN training method 
The impact of the MLS was examined based on length of training time. This includes retraining monthly using cumulative data, as well as by clearing the MLS's memory each month for retraining. This comparison will give insight into the impact of seasonal changes on the performance of the MLS, as the data in Figure 3-6 indicates potential changes in accuracy. The lowest points in training accuracy are associated with months of the year in which the variability in weather is highest, and patterns are therefore more difficult for the MLS to determine.

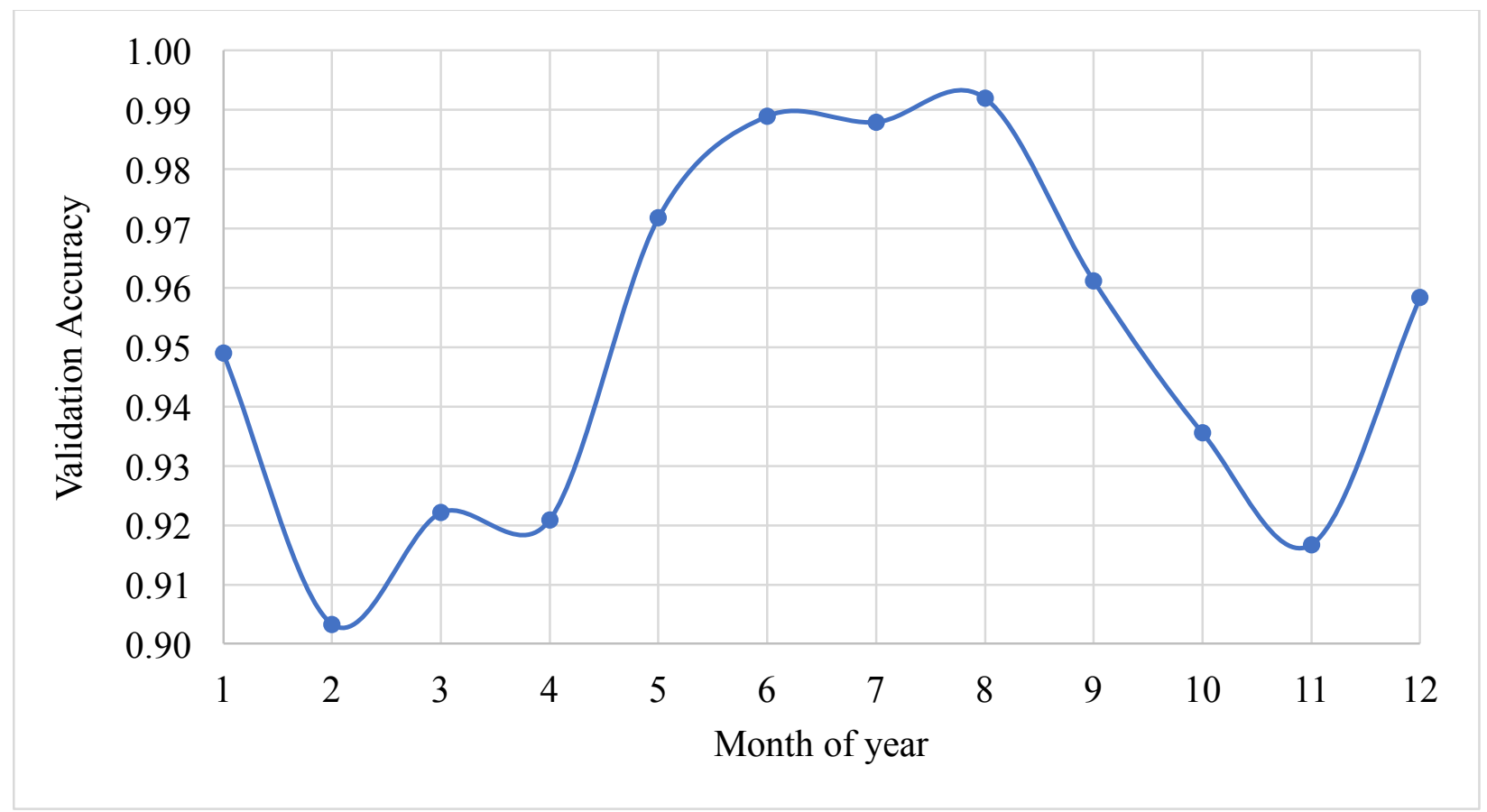

Figure 3-6: Training validation accuracy of the MLS for each month of the year

\subsubsection{Predictive Control Case Study Description}

To complement the MLS, which utilizes historical data to make decisions, predictive control was investigated. While the predictive controller developed utilizes historical data as well, it also uses weather forecasts to predict future on-site energy production and consumption.

The most interesting component of predictive control is the error associated with weather forecasts and the models used by the controller. Simply put, the question to be asked is: does the error in forecasting and modelling hinder the performance of the controller in any way? Therefore, in order to truly understand the potential of predictive control, actual weather forecast data must be used. 
CAnMETEO software has been utilized to provide weather forecast data. Developed by NRCan, CAnMETEO provides hourly weather forecasts with very high location resolution. The software was used to provide weather data forecasts up to 48 hours in the future.

A LabVIEW application was developed that ran the CanMETEO software at 6-hour intervals. This application was run for just over 1 month (November $7^{\text {th }}-$ December $\left.15^{\text {th }}, 2016\right)$ in order to collect forecast data for future analysis, as well as determine whether the software was stable enough to run an experimental control system. After 1 month of data collection, just over $40 \%$ of the forecast files collected were composed entirely of NULL values. The longest period of clean, continuous forecast data spanned a 10-day period in November. At this point in time, it is not clear whether this was caused by an issue with the LabVIEW software, the CanMETEO software, or a communication issue with the source server. Nevertheless, a real-world controller could not be implemented due to inconsistent operation. Instead, a case study was performed using the 10 days of continuous forecast data.

While the forecast data contained a variety of weather-related parameters, only ambient temperature, wind speed, and solar radiation data was used for predictive decisions. These parameters were used to train ANN models of HVAC loads, and renewable generation. After various trials, it was determined that this type of black box modelling cannot accurately represent appliance or hot water loads. Therefore, predictive control was performed only on HVAC loads in a home. The ANNs for predictive control all used Levenberg-Marquardt backpropagation training, and their inputs and outputs are summarized in Table 3-6.

Table 3-6: Predictive ANN inputs and outputs

\begin{tabular}{|c|c|c|}
\hline Predictive ANN Model & Inputs & Outputs \\
\hline HVAC ANN & $\begin{array}{ll}\text { - } & \text { Ambient temperature } \\
\text { - } & \text { Irradiance (DNI, GHI, diffuse) } \\
\text { - } & \text { Wind speed }\end{array}$ & $\begin{array}{l}\text { - HVAC power } \\
\text { consumption }\end{array}$ \\
\hline PV ANN & $\begin{array}{ll}\text { - } & \text { Ambient temperature } \\
\text { - } & \text { Irradiance (DNI, GHI, diffuse) } \\
\text { - } & \text { Wind speed }\end{array}$ & - $\quad$ PV power generation \\
\hline
\end{tabular}


Similarly, the wind speed data provided by the software did not accurately reflect wind conditions at the Living City Campus, and an accurate predictive model of a wind turbine could not be developed. Wind turbines were therefore neglected from the predictive control case study. In the end, two ANN models were developed for predictive control use: an HVAC electrical load model, and a solar PV generation model. These models were trained using simulation data, explained in Chapter 4.

Once forecast data is obtained, and used to estimate future electrical consumption and generation, the GA optimization protocol was applied to the load and generation profiles. The GA assumed the use of a $25 \mathrm{kWh}$ battery, an appropriate size for the HVAC-only load case. This generates battery state profiles that have been optimized, while taking into account the future state of the system

In the real-world, the predictive controller's operation would follow the following steps:

1. Acquire and log hourly averaged HVAC, solar PV, and weather data,

2. At predefined intervals (every midnight), or if data is outside of current training range, retrain the ANN models using data from the past day,

3. Collect weather forecast data,

4. Apply weather forecast data to newly updated ANN models, producing load and generation data,

5. Run GA optimization tool to produce optimal battery management profiles for use over the following 24 hours.

A case study was performed, and the described predictive control protocol was simulated over the 10-day period for which forecast data was available. All other EMSs (except for transactive control) were run on this same 10-day period, under the same conditions, in order to compare their operation on the same basis. The EMSs were compared on the basis of variable cost and energy consumption. 


\subsubsection{Transactive Energy Management System Description}

One of the adaptive control schemes investigated in this work is a Transactive Energy Management System (TEMS). This is an agent-based control scheme using a centralized controller and distributed agents. It is a highly scalable system, whose operation only requires the main controller, and is not dependent on any single agent. The transactive energy framework represents general guidelines for the application of a transactional-based energy system; there are countless different application methods for this control structure. This work represents one interpretation of the transactional energy framework for building energy application, and by no means represents transactional energy in its entirety.

The TEMS in this work is comprised of three main device types: loads, DGs, and battery banks. Agents, the smart communication devices that facilitate the entry of an energy consuming or producing device into the marketplace, are responsible for monitoring their respective devices, and communicating their bid to the main controller - the Energy Marketplace. The ways in which the Marketplace and each agent operate are described in the section below.

\subsubsection{Energy Marketplace}

The Energy Marketplace is the environment in which transactional energy contracts are negotiated. Bids from participant agents are collected at discrete time intervals and sorted based on whether they contribute to either the supply or the demand of the system. The bid of each agent is made up of the following four components:

- Unique Bidder ID

- Bid Price $(\$ / \mathrm{kWh})$

- $\operatorname{Bid}$ Amount $(\mathrm{kW})$

- Duration of Contract

In reality, it does not matter if the bid amount is in $\mathrm{kW}$ or $\mathrm{kWh}$, as long as the Marketplace evaluates bids at fixed time intervals. If the Marketplace evaluates bids every minute, then agents will always be requesting their bid amount in $\mathrm{kW}$ for at least one minute, which can be converted into $\mathrm{kWh}$ values. Using $\mathrm{kW}$ merely simplifies the units used in calculations. 
Once all the bids have been collected the marketplace constructs supply and demand curves, mimicking those which are common to free market economic systems. Supply curves are arranged in ascending order according to price, ensuring that producers that are willing to sell their energy for the lowest price do so before more costly production. Demand curves are arranged in descending order according to price, ensuring that consumers with higher needs have their load requirements satisfied before those with less urgency.

Figure 3-7 shows an example of a marketplace evaluation. This example shows five loads (L1 L5), one DG (DG1) and the grid. Each evaluation determines the point at which the supply and demand curves meet, calculating the market clearing price. This is the price at which all energy is traded - regardless of initial bid price - during a given marketplace evaluation period.

In Figure 3-7 (a), the supply of renewable energy is relatively low, and only the loads with the greatest needs are granted their bid requests. The market clearing price is calculated to be 0.11 $\$ / \mathrm{kWh}$, the off-peak TOU price of the grid. After this evaluation L1, L2, and L3 are awarded demand bid contracts, supplied by DG1 and the grid. Figure 3-7 (b) shows the same system at the following time step. Renewable energy production has increased, pushing the supply curve to the right. This increase in supply lowers the market clearing price to $0.05 \$ / \mathrm{kWh}$, allowing loads with lower bids to come online. At this point in time, L4 can power on, supplied entirely by renewable energy.

Not pictured in Figure 3-7 are critical loads - those that must have their demand satisfied. In this work, the definition of critical load has been relaxed from its conventional meaning to mean any non-HVAC load. Without detailed occupancy preferences for every individual appliance, it is impossible to accurately determine the need-of-operation, and therefore a bid amount. Therefore, all non-HVAC loads have been neglected from the TEMS. Initially this was not the case, but after many trials under the assumption that non-HVAC loads were critical loads, it was determined that this neither added advantages nor disadvantages to the system, and merely complicated simulations and marketplace evaluation. 


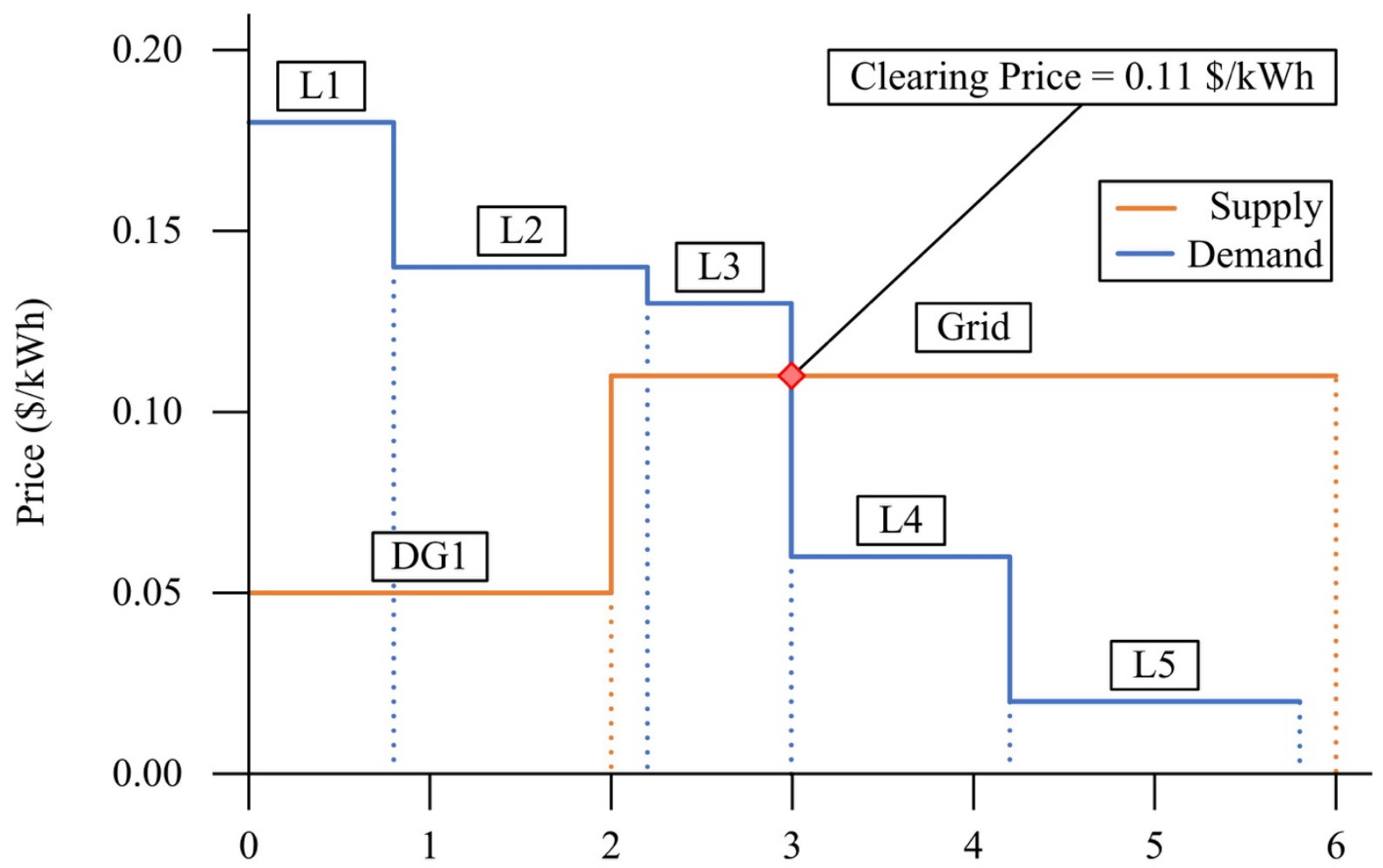

(a)

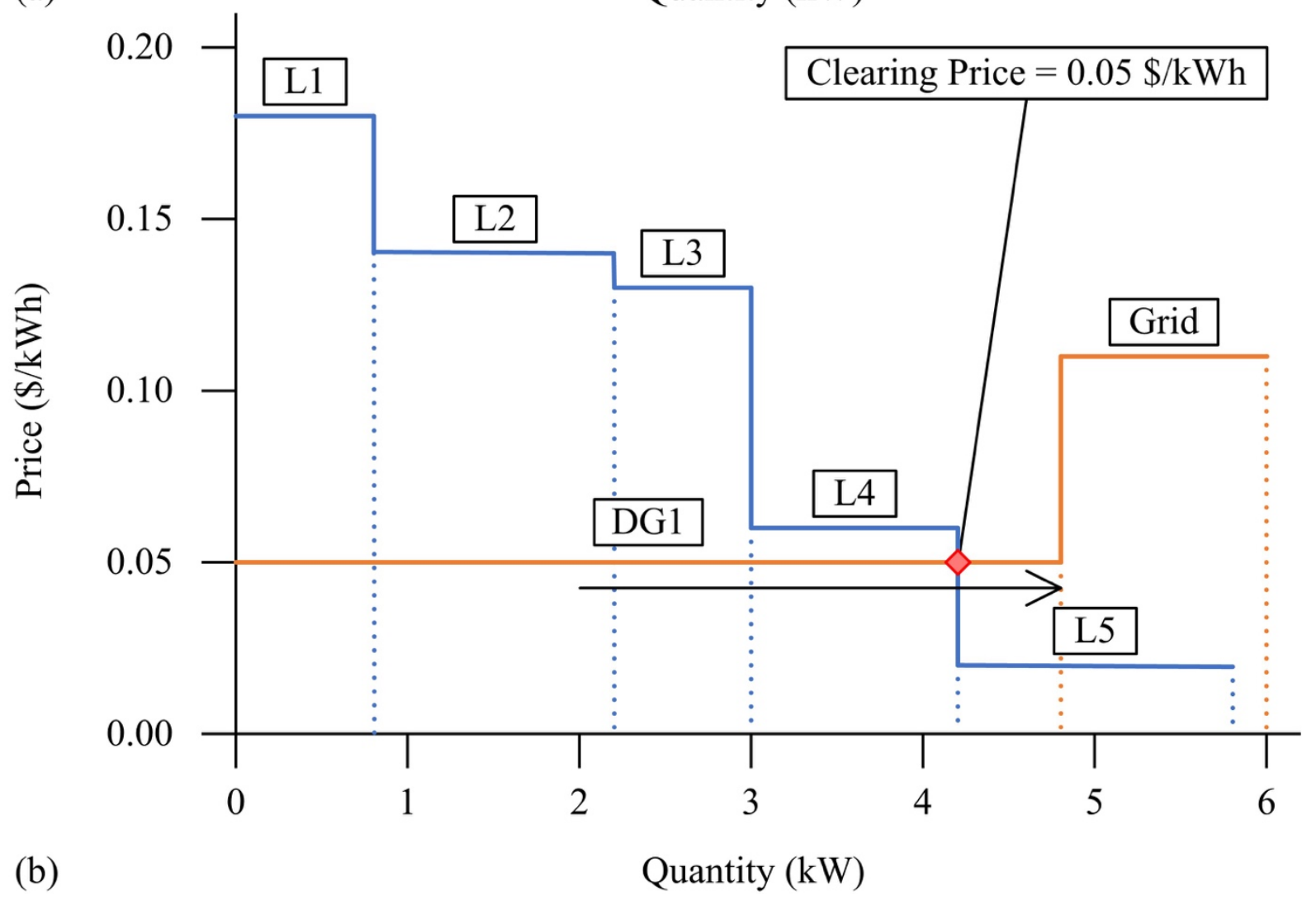

Figure 3-7: An example of supply and demand evaluation in the Energy Marketplace 


\subsubsection{ASHP Bidding}

The only loads used in the TEMS were the ASHPs used to heat and cool the houses. Since this only represents one load per household, some simulations involved multiple dwellings in order to introduce more demand into the Energy Marketplace. The bid of an ASHP can be linked directly to thermal comfort - specifically to deviation from the zone set point temperature. Figure 3-8 shows a sample of a thermostat bid curve relative to different prices of electricity.

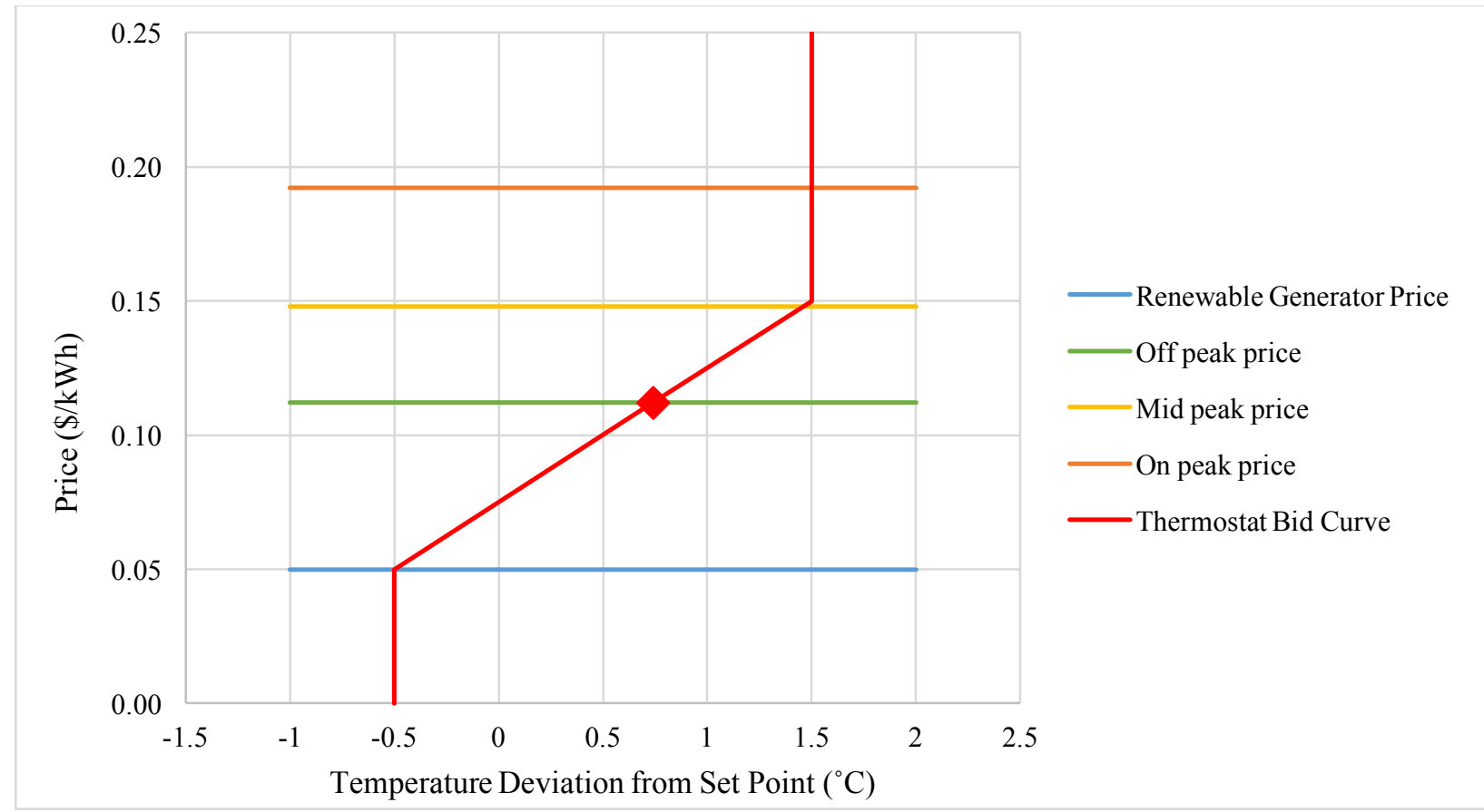

Figure 3-8. Sample ASHP bid curve relative to different energy prices.

The intersection of the bid curve with the various energy price curves indicates the point at which the ASHP is willing to pay a sufficient price for the respective energy source. Therefore, the slope of the curve reflects the thermal comfort preferences of the occupant; a steeper bid curve indicates that a homeowner is willing to pay a greater amount to maintain their zone at a certain temperature, than a homeowner who has set a shallower bid curve. An analysis was performed investigating the impact of slope of bid curve on annual energy consumption and zone temperature.

Various test trials conducted in this work indicated that the exact shape of the bid curve (polynomial, exponential, etc.) had a minimal effect on annual energy consumption and cost, and straight-line bid curves were used for all calculations. 


\subsubsection{DG Bidding}

Bid amounts for DGs were fixed at a constant price based on type of generator. Table 3-7 summarizes the bid price used for all solar supply bids.

Table 3-7: Bid prices for solar PV in a TEMS

\begin{tabular}{|c|c|}
\hline Type of Generator & Bid Price (\$/kWh) \\
\hline Solar PV & 0.05 \\
\hline
\end{tabular}

This price is of course not representative of the actual cost of solar energy. This amount has been chosen arbitrarily such that it is lower than the off-peak TOU electricity price, incentivizing its use. While the bid price is relatively low, the actual price at which all energy is traded after each marketplace evaluation is the market clearing price. While the detailed implications of this fact are not investigated in this work, the actual prices at which solar energy is traded has been identified.

\subsubsection{Battery Bank Bidding}

The bidding process for the battery bank is much more complicated than for a thermostat or DG; the battery bank can bid a variable price, for both the supply (discharging) and demand (charging) curves. Additional control logic was therefore required in order to properly integrate the battery bank as an active bidding agent.

A custom fuzzy interference system (FIS) was developed and implemented in order to make the bidding decisions for the battery bank. This fuzzy logic controller was developed using the MATLAB Fuzzy Logic Designer Toolkit.

It was decided to use two different inputs for the battery FIS: current battery capacity, current net load. Based on its control rules, the FIS then output the price of battery energy in either a negative (demand) or positive (supply) amount. The use of fuzzy logic fits the system requirement well, since it allows the controller to be designed in more abstract terms. It allows the battery bank's energy content to be described in such terms as "Very Low" or "High", which alleviates the need to define strict numerical limits on what may be defined as "Very Low" battery energy content. Similar work on battery charging/discharging control has been investigated in the past (Arcos 
Avilés et al., 2012), and this work has adapted the previously developed principles for a transactive energy application.

The membership functions (MFs) for the battery FIS were chosen to best represent the state of the battery, and the surrounding system that it serves. Figure 3-9 to Figure 3-11 show the two input and single-output MFs.

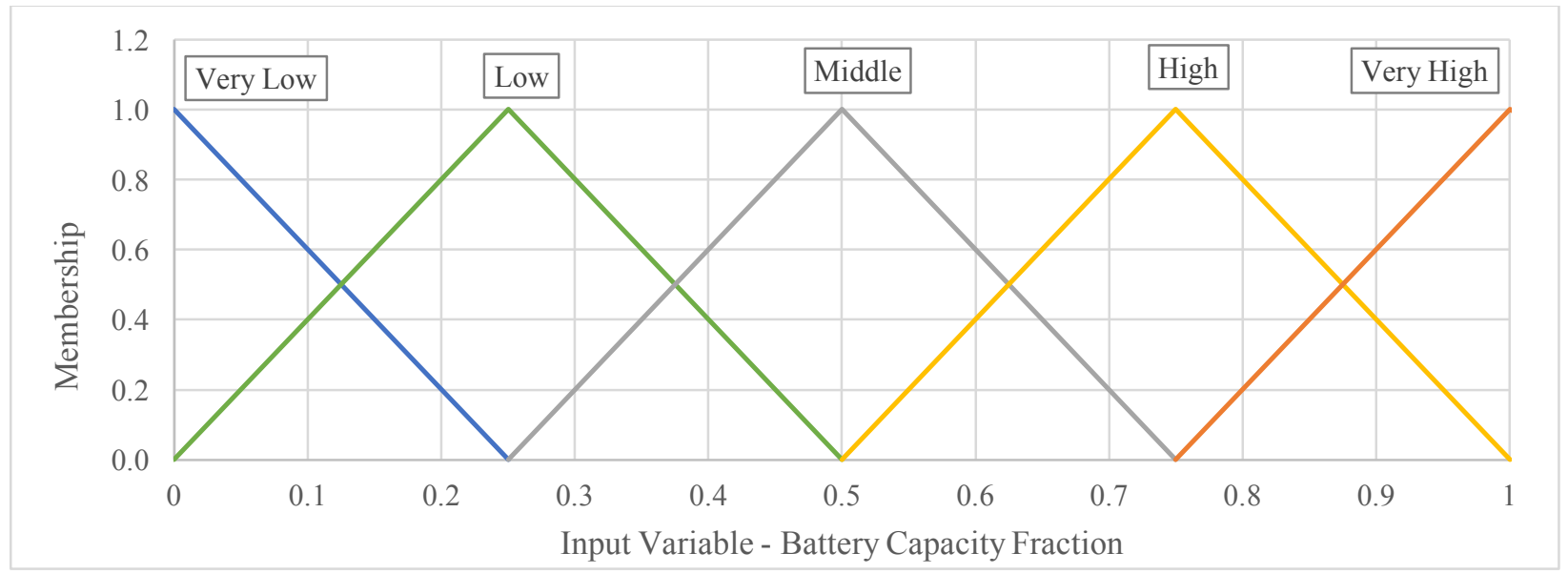

Figure 3-9: Battery FIS input membership function - Battery capacity fraction

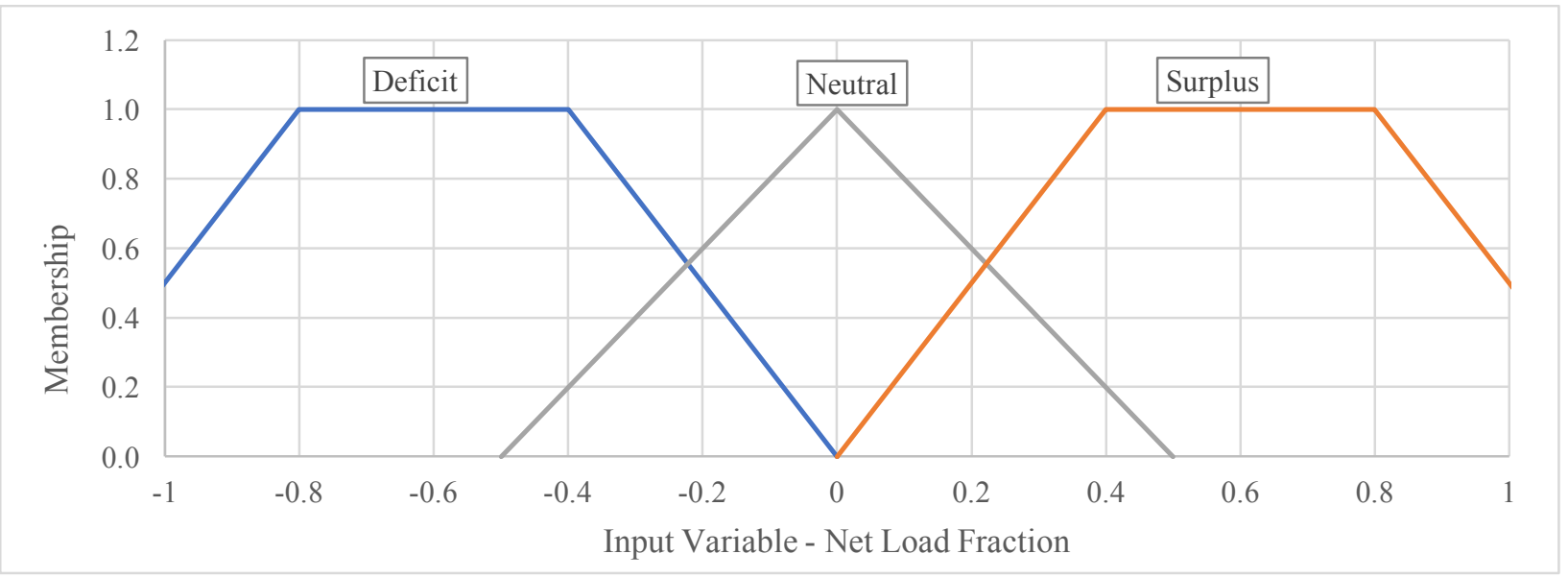

Figure 3-10: Battery FIS input membership function - Net load fraction 


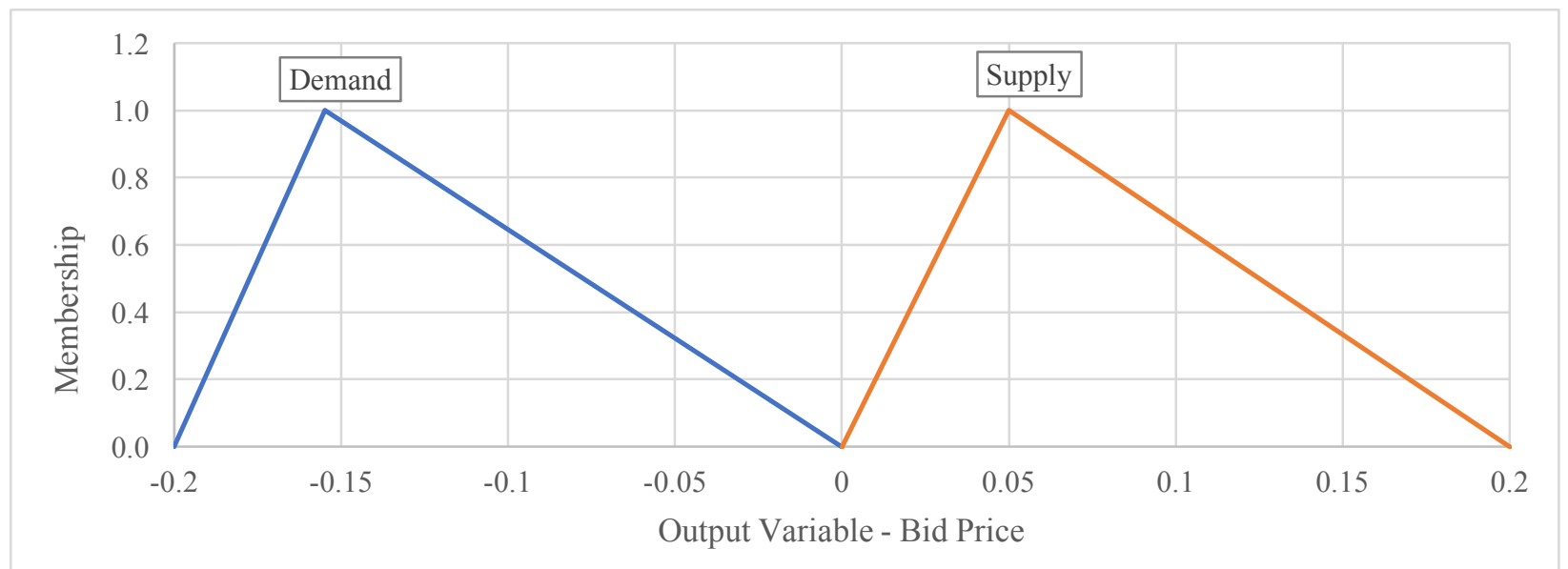

Figure 3-11: Battery FIS output membership function - Bid price

The battery capacity fraction is defined as the current ratio of the battery's energy content to its total capacity - the inverse of depth of discharge (DOD) - and ranges from 0 to 1 . The decision to evenly span the MFs for this input parameter was arbitrary.

The net load fraction (NLF), calculated in Equation 3-1, is defined as the current net load divided by the battery's maximum charge/discharge rate. For all calculations, it was assumed that the maximum charge/discharge rate was $6 \mathrm{~kW}$. If the NLF is negative, it indicates that there is a deficit in on-site generation, while a positive value indicates a generation surplus.

$$
N L F=\left\{\begin{aligned}
-1, & \text { if } \frac{P_{D G, \text { tot }}-P_{\text {load }, \text { tot }}}{P_{\text {inverter,max }}} \leq-1 ; \\
1, & \text { if } \frac{P_{D G, \text { tot }}-P_{\text {load }, \text { tot }}}{P_{\text {inverter,max }}} \geq 1 ; \\
\frac{P_{D G, \text { tot }}-P_{\text {load,tot }},}{P_{\text {inverter,max }}}, & \text { otherwise. }
\end{aligned}\right.
$$

Since the ASHP's load is almost always entirely below $6 \mathrm{~kW}$, the conditionality in Equation 3-1 was only implemented if additional loads were brought into the TEMS.

Initially the NLF evenly covered the input range, mirroring the battery capacity fraction MFs. However, after various trials, it was determined that the MFs shown in Figure 3-10 best 
characterized the system. Additionally, three MFs, as opposed to five, sufficiently achieved the required results, while reducing the complexity of the rules used in the battery FIS.

The output variable defined whether the battery would bid towards the supply or demand curve, and the amount that it would bid. A negative value indicates a bid for the demand curve, while a positive value indicates a bid for the supply curve. The shapes of the MFs shown in Figure 3-11 were modified such that a demand bid would never exceed the mid-peak TOU price, and the supply bid would lie just below the price of off-peak electricity.

After various trials, it was determined that without separate day and night control, the battery would rarely bid enough to charge from the grid overnight. Two different sets of rules were then developed to help the battery recharge from the grid when appropriate. Based on trial and error, it was found that the FIS rules summarized in Table 3-8 and Table 3-9 yield the best results at this point in time. This rule scheme has only been somewhat optimized, and is expected that a better bidding structure may exist. Based on this set of rules, the battery capacity could be reduced from five to two or three sets, and ignored altogether during the day.

Table 3-8: Battery FIS control rules - Daytime

\begin{tabular}{|c|c|c|c|c|c|}
\hline & \multicolumn{5}{|c|}{ Battery Capacity Fraction } \\
\hline NLF & Very Low & Low & Mid & High & Very High \\
\hline Deficit & Supply & Supply & Supply & Supply & Supply \\
\hline Zero & Supply & Supply & Supply & Supply & Supply \\
\hline Surplus & Demand & Demand & Demand & Demand & Demand \\
\hline
\end{tabular}

Table 3-9: Battery FIS control rules - Nighttime

\begin{tabular}{|c|c|c|c|c|c|}
\hline & \multicolumn{4}{|c|}{ Battery Capacity Fraction } \\
\hline NLF & Very Low & Low & Mid & High & Very High \\
\hline Deficit & Demand & Demand & Demand & Supply & Supply \\
\hline Zero & Demand & Demand & Demand & Supply & Supply \\
\hline Surplus & Demand & Demand & Demand & Demand & Demand \\
\hline
\end{tabular}




\subsection{Methodology Summary}

Figure 3-12 summarizes the overall research methodology in the form of a flow chart.

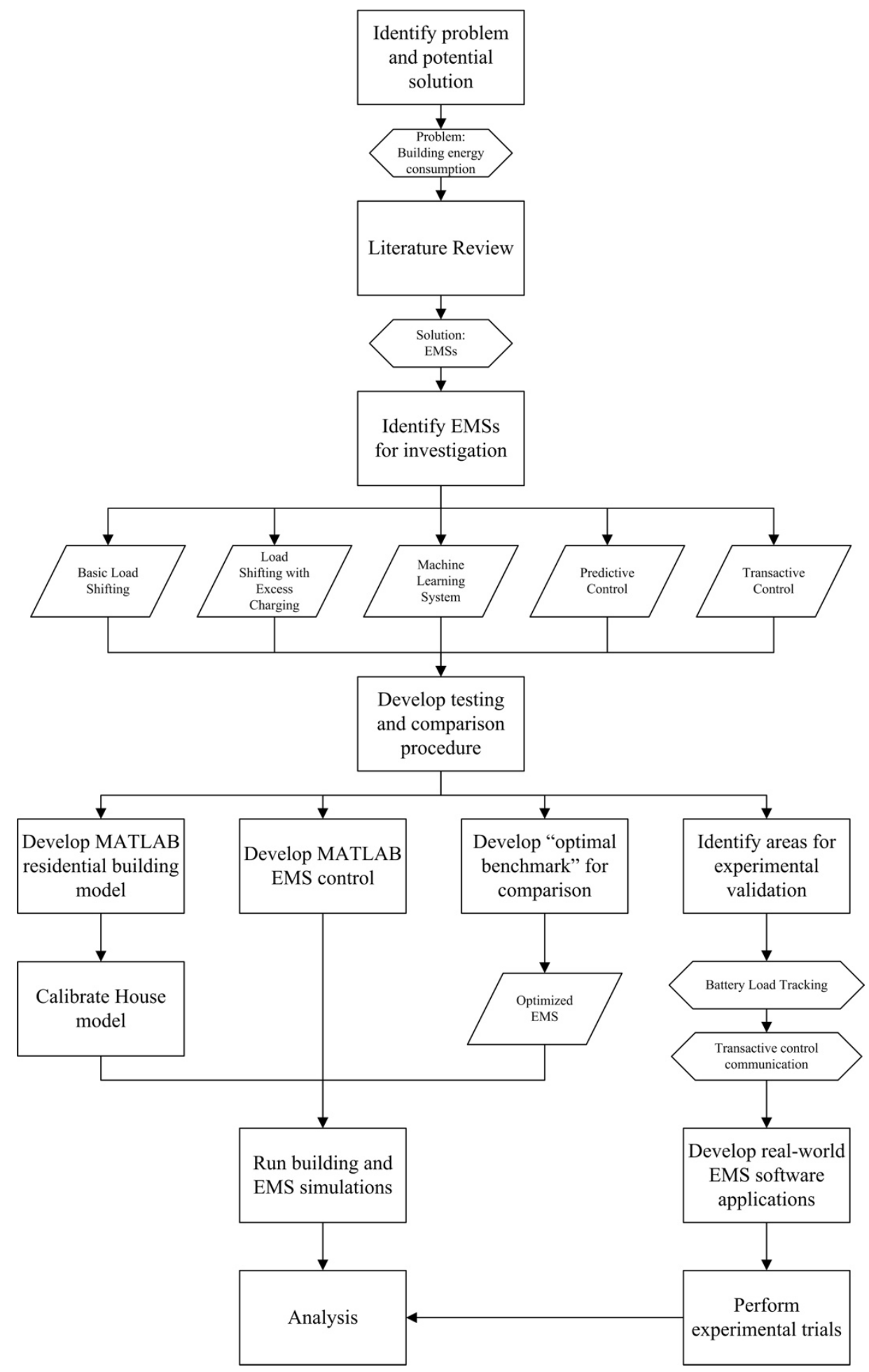

Figure 3-12: Research methodology flow chart 


\section{House Description and Model Summary}

Energy management control schemes for buildings can issue controls to loads, DGs, and energy storage devices. However, having these three arms of control compounds the difficulty of the problem. For the EMSs investigated in this work, certain simplifications were made. Firstly, it was assumed that the only possible control over DGs was zero-feedback throttling; when renewable generation exceeds the on-site load the generation is throttled back, via the inverter, to match the load such that no electricity is exported to the grid. This simplifies energy calculations, and neglects any possible revenue associated with feed-in-tariff (FIT or microFIT) or net metering contracts, allowing any changes in operating cost to be attributed entirely to the EMS.

The second simplification was to treat most household loads as critical loads. Without estimating occupancy preferences for every individual appliance, it is impossible to impose flexibility over, for example, the dryer or lights in a home. There was one exception - the heating and cooling systems used in homes were treated as flexible loads. Since the operation of these loads can be linked directly to thermal comfort, expressed as a range of comfortable conditions, they inherently have flexibility in their operation.

The final simplification was that if it was decided to charge the battery bank from the grid alone, the battery would be charged at the inverter/charger's full charge rate. However, if a decision was made to charge the battery from renewables, it would be charged with the available amount of power. Using the full charge rate from the grid negates the need to calculate the optimal charge rate from the grid at every possible moment in time.

While the battery's state of charge can be easily calculated based on energy withdrawn or injected, the matter of determining the impact of the HVAC system on a home is much more complicated. An accurate building model is essential in order to produce meaningful results in terms of the application of an EMS controlling HVAC loads.

A residential house model has been developed in $M A T L A B$. This programming language, as opposed to building simulation software, was used because of its powerful optimization and AI capabilities. Having a building model in MATLAB ensured that any possible control action could 
be applied, without having to worry about bending to the requirements of other building simulation software.

The model is based on the Archetype Sustainable House A due to the vast amount of available data related to its operation. A physics-based grey box model has been developed and calibrated to represent the operation of the house and all its relevant internal components. This means that while some parameters use their real-world values, certain characteristics have been modified via calibration procedure such that the model accurately reflects real-world operation. The model description, along with inputs, outputs, and calibration procedure, are described in the following sections.

\subsection{Site Description}

The Archetype Sustainable House (ASH), located at the Living City Campus in Vaughan, Ontario, is composed of two semi-detached model homes named House A and House B. Originally developed from an architectural design competition for sustainable buildings, today the ASH acts as a test beds for all forms of sustainable building technologies and renewable energy.

The houses are constructed from sustainable building materials, use heat recovery for both water and air, heat and cool using high-efficiency HVAC systems, and more. House A is utilized sustainable technologies that are commercially available today, while House B showcases more experimental and innovative technologies that are only just entering the market. Figure 4-1 shows the ASH at the Kortright Centre for Conservation. Table 4-1 summarizes the major structural features of both House A and B.

Adjacent to the ASH is a solar PV test field and a small-scale wind turbine testing facility. The PV test site is a research facility features numerous different PV modules for demonstration, monitoring, and comparison purposes. Solar radiation is measured using various measurement devices, including pyranometers, silicon cell photodiodes, and a Kipp \& Zonen Solys 2 Tracker, to ensure verify data repeatability. Detailed weather data is monitored using a Lufft WS600 weather station. The nearby small-scale wind turbine testing facility features a variety of wind turbines under test. Wind speed, direction, and air temperature are measured at various heights on 
a nearby meteorological (MET) tower. The ASH, solar PV test field, and wind turbine testing facility are all monitored using a distributed Data Management System (DMS).

Table 4-1: Archetype Sustainable House structural features (Safa, Fung, \& Kumar, 2015)

\begin{tabular}{|c|c|c|}
\hline Structural features & House A & House B \\
\hline Basement walls & RSI 3.54 (R20) & RSI 3.54 (R20) \\
\hline Basement slab & RSI 1.76 (R10) & RSI 1.76 (R10) \\
\hline Above grade walls & RSI 5.64 (R32) & RSI 5.64 (R32) \\
\hline Windows & $2.19 \mathrm{~W} / \mathrm{m}^{2} \mathrm{~K}$ & $1.59 \mathrm{~W} / \mathrm{m}^{2} \mathrm{~K}$ \\
\hline Roof & RSI 7 (R40) & RSI 7 (R40) \\
\hline Overall UA value & $160 \mathrm{~W} / \mathrm{K}$ & $172 \mathrm{~W} / \mathrm{K}$ \\
\hline $\mathrm{ACH} @ 50 \mathrm{~Pa}$ & 1.204 & 1.091 \\
\hline \multicolumn{3}{|l|}{ Floor areas $-m^{2}\left(f t^{2}\right)$} \\
\hline Basement & $86.95(936)$ & $86.95(936)$ \\
\hline First floor & $86.95(936)$ & $86.95(936)$ \\
\hline Second floor & $86.95(936)$ & $60.19(636)$ \\
\hline Third floor & $83.60(900)$ & $86.95(936)$ \\
\hline Total & $344.0(3708)$ & $321.0(3444)$ \\
\hline \multicolumn{3}{|c|}{ Zone volumes $-m^{3}\left(f t^{3}\right)$} \\
\hline Basement & $234.0(8264)$ & $234.0(8264)$ \\
\hline First floor & $292.5(10296)$ & $292.5(10296)$ \\
\hline Second floor & $238.5(7840)$ & $238.5(7840)$ \\
\hline Third floor & $222.0(7840)$ & $271.8(9600)$ \\
\hline Total & $986(34,824)$ & $1036(36,584)$ \\
\hline
\end{tabular}

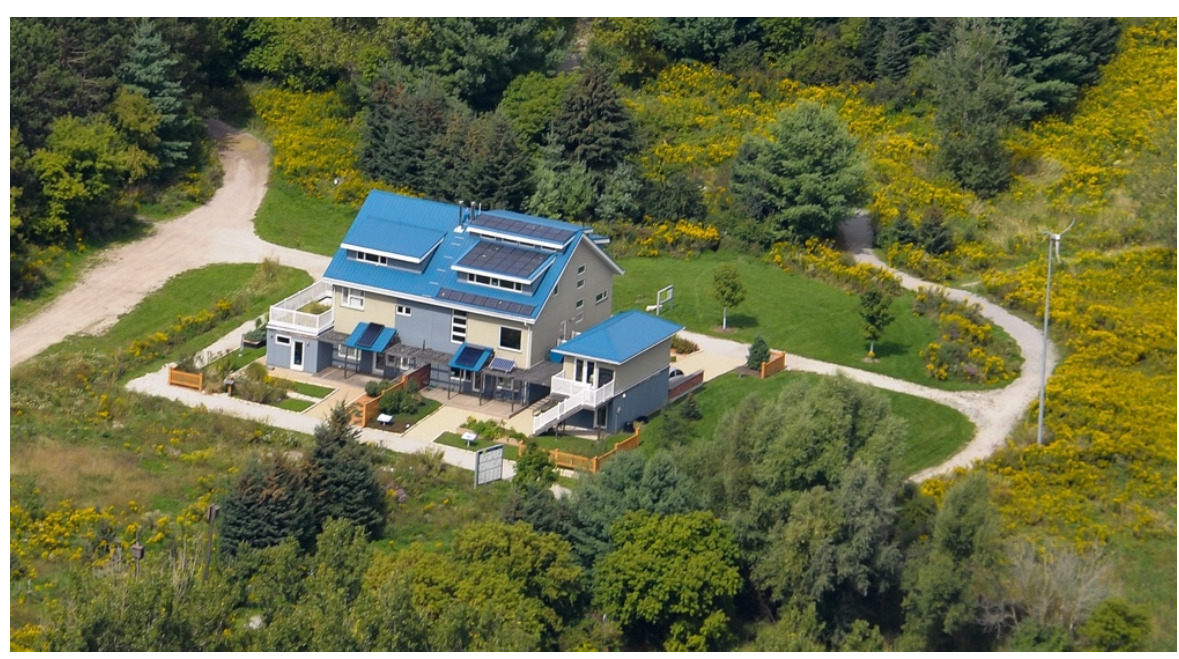

Figure 4-1: The Archetype Sustainable House A (left) and House B (right) at the Living City Campus 


\subsubsection{Data Management System}

The DMS used at the Living City Campus is a set of custom distributed monitoring applications designed and implemented using National Instruments (NI) hardware and software (LabVIEW), the DMS is responsible for all on-site data acquisition (DAQ). The DMS is responsible for 500 monitoring points, logging at $1-5$ second intervals. All experimental work was integrated into the existing DMS.

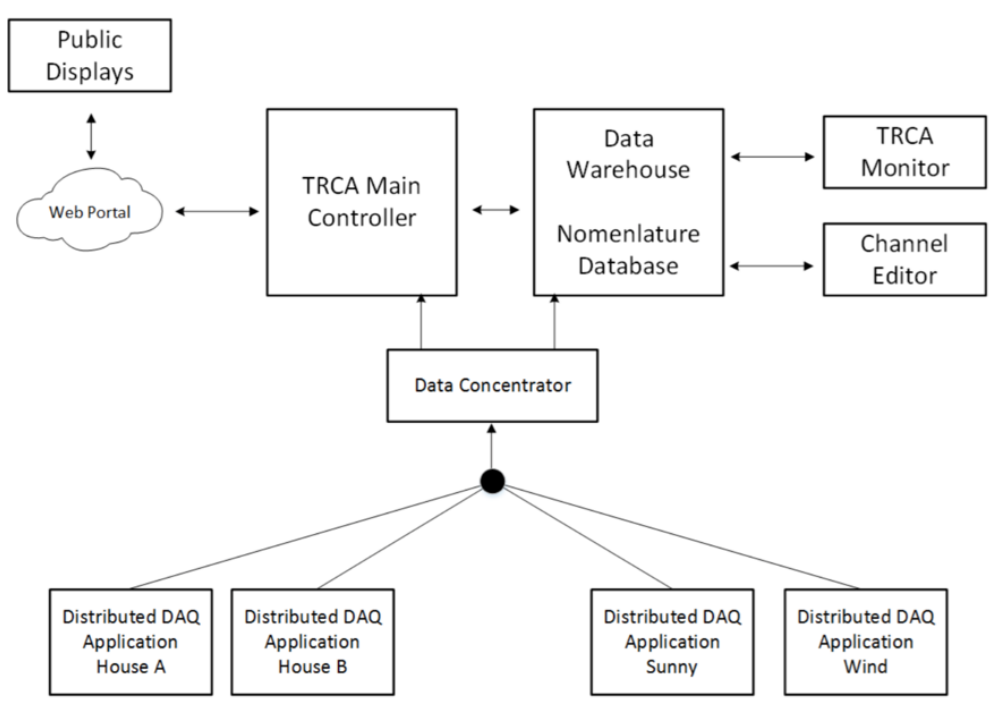

Figure 4-2: Data Management System hierarchy (Saxena, 2017)

\subsubsection{Mechanical and Renewable Energy Systems}

The relevant mechanical systems used in Houses A and B are summarized in Table 4-2. Simulations were based on the heating and cooling, DHW, and ventilation systems used in House A, and the renewable energy systems of House B.

Not included in Table 4-2 is the energy storage capacity at the Living City Campus. Located adjacent to the ASH, in the wind test facility control building, is a $1600 \mathrm{Ah}$ (50 VDC) lead-acid battery bank. This energy storage device is controlled by a Xantrex XW $6048 \mathrm{~kW}$ inverter/charger. A Connext ComBox allows for communication and control of the inverter/charger via Modbus communication protocol, such that the battery may be remotely controlled. 
Table 4-2: Archetype Sustainable House system summary

\begin{tabular}{|l|c|c|}
\hline \multicolumn{1}{|c|}{ System } & House A & House B \\
\hline Solar PV & None & $4.08 \mathrm{~kW}$ \\
\hline Wind Turbine & None & $2.4 \mathrm{~kW}$ \\
\hline Heating and Cooling & $\begin{array}{c}\text { Variable capacity ASHP } \\
\text { packaged with AHU }\end{array}$ & $\begin{array}{c}\text { GSHP with horizontal and } \\
\text { vertical loops, desuperheater, } \\
\text { and buffer tank }\end{array}$ \\
\hline Domestic hot water & $\begin{array}{c}\text { Outdoor air source heat pump } \\
\text { water heater and auxiliary } \\
\text { mini gas boiler }\end{array}$ & $\begin{array}{c}\text { Indoor air source heat pump } \\
\text { water heater (ASHPWH) }\end{array}$ \\
\hline Ventilation & $\begin{array}{c}\text { Heat recovery ventilator } \\
\text { (HRV) }\end{array}$ & $\begin{array}{c}\text { Energy recovery ventilator } \\
\text { (ERV) }\end{array}$ \\
\hline
\end{tabular}

\subsection{House Model Inputs}

The Archetype Sustainable House A was used as the basis for the model developed in this work due to the availability of data regarding its HVAC operation. The model uses real-world weather data and house parameters as inputs.

\subsubsection{Weather Data}

Weather data collected at the Living City Campus was used for all simulations. This data was critical for two reasons. First, it ensured that an accurate calibration of the house model could be performed, since the data was collected at the location of the ASH-A. Second, the data was available at very high resolutions (up to 1 second), allowing the simulation to capture the variability of real-world weather conditions. The weather parameters used as model inputs are summarized in Table 4-3.

Table 4-3: Weather data used for simulations

\begin{tabular}{|l|c|c|}
\hline Parameter & Unit & Sensor \\
\hline Ambient Temperature & ${ }^{\circ} \mathrm{C}$ & Lufft WS 600 \\
\hline Humidity Ratio & $\mathrm{g}_{\text {water }} / \mathrm{g}_{\text {air }}$ & Lufft WS 600 \\
\hline Wind Speed & $\mathrm{m} / \mathrm{s}$ & Lufft WS 600 \\
\hline Direct Normal Irradiance (DNI) & $\mathrm{W} / \mathrm{m}^{2}$ & Kipp \& Zonen Solys 2 Tracker \\
\hline Diffuse Irradiance & $\mathrm{W} / \mathrm{m}^{2}$ & Kipp \& Zonen Solys 2 Tracker \\
\hline Global Horizontal Irradiance (GHI) & $\mathrm{W} / \mathrm{m}^{2}$ & Kipp \& Zonen Solys 2 Tracker \\
\hline
\end{tabular}




\subsubsection{House Data}

The actual specifications of the ASH-A were used as model inputs. However, some parameters were modified in order to calibrate the grey-box house model. A summary of the fixed versus calibrated ASH-A specifications is presented in Table 4-4. Values specific to wall orientation are summarized in Table 4-5.

Table 4-4: Summary of fixed and calibrated parameters for the ASH-A model

\begin{tabular}{|l|c|c|}
\hline Parameter & Fixed/Calibrated & Value \\
\hline Wall overall heat transfer coefficient & Calibrated & - \\
\hline Wall exterior solar absorption & Fixed & 0.9 \\
\hline Floor interior solar absorption & Calibrated & - \\
\hline Wall thickness & Calibrated & - \\
\hline Wall thermal conductivity & Calibrated & - \\
\hline Wall density & Calibrated & - \\
\hline Wall heat capacity & Calibrated & - \\
\hline Window overall heat transfer coefficient & Calibrated & $0.56(\mathrm{NRCan}, 2017)^{20}$ \\
\hline Window solar heat gain coefficient & Fixed & $986 \mathrm{~m}^{3}$ \\
\hline Zone volume & Fixed & \\
\hline
\end{tabular}

Table 4-5: Orientation-specific ASH-A values (Alibabaei, 2016)

\begin{tabular}{|c|c|c|c|}
\hline Wall Direction & Azimuth & Wall Area & Window Area \\
\hline North & 180 & $39.7 \mathrm{~m}^{2}$ & $9.6 \mathrm{~m}^{2}$ \\
\hline East & -90 & $65.3 \mathrm{~m}^{2} *$ & $0.0 \mathrm{~m}^{2}$ \\
\hline South & 0 & $39.7 \mathrm{~m}^{2}$ & $11.0 \mathrm{~m}^{2}$ \\
\hline West & 90 & $65.3 \mathrm{~m}^{2}$ & $10.3 \mathrm{~m}^{3}$ \\
\hline Roof & 0 & $107.3 \mathrm{~m}^{2}$ & - \\
\hline Floor & 0 & $80.2 \mathrm{~m}^{2}$ & - \\
\hline
\end{tabular}

*The east wall is a shared wall with House B. 


\subsection{Weather-Based Calculations}

\subsubsection{Solar Radiation Calculations}

Solar radiation calculations are critical, not only for determining the thermal response of a building, but also for the estimation of solar PV generation. Actual solar irradiance data, collected at the Living City Campus, was used for all solar calculations. This data includes the direct normal irradiance (DNI), $I_{D N I}$, the diffuse horizontal irradiance (diffuse), $I_{\text {diff }}$, and the global horizontal irradiance (GHI), $I_{G H I}$, all in $\mathrm{W} / \mathrm{m}^{2}$. These values are recorded at 1 second intervals on a Kipp \& Zonen Solys 2 Tracker. However, the data only applies to the orientation of the tracker itself, and must be translated, using solar geometry, such that it may be applied to surfaces with different orientations.

In order to determine the amount of solar radiation incident on a surface, the angle of incidence of solar radiation must be determined. This angle must be calculated for every surface contacted by solar radiation. All solar radiation calculations used in this work are standard in the solar energy industry (Duffie \& Beckman, 2006).

\subsubsection{Incidence Angle}

The incident solar radiation on a given surface is proportional to the cosine of the angle of incidence, $\theta$, on that surface. Numerous angles - functions of location, orientation, and time of day and year - are required to determine the angle of incidence. Table 4-6 summarizes all angles used for calculations, while Figure 4-3 gives a visual representation of some of the relevant angles. 


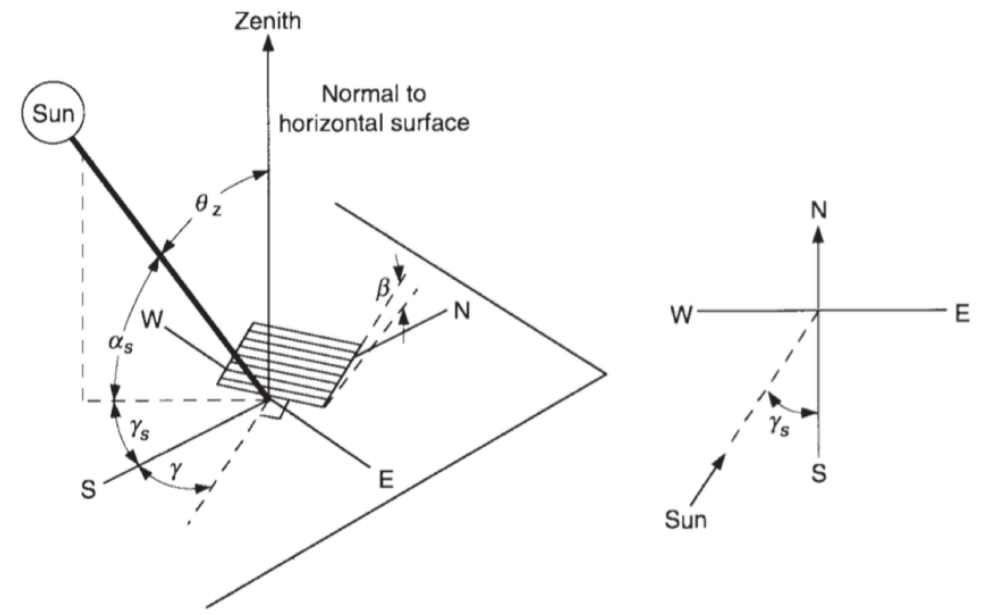

Figure 4-3: Relevant solar calculation angles (Duffie \& Beckman, 2006)

Table 4-6: Relevant solar angles (Duffie \& Beckman, 2006)

\begin{tabular}{|l|c|l|}
\hline \multicolumn{1}{|c|}{ Angle } & Symbol & \multicolumn{1}{|c|}{ Description } \\
\hline Latitude & $\delta$ & $\begin{array}{l}\text { The location north or south of the equator, with } \\
\text { north being positive. }\end{array}$ \\
\hline Declination & $\beta$ & $\begin{array}{l}\text { The position of the sun at solar noon with respect } \\
\text { to the plane of the equator, with north being } \\
\text { positive. }\end{array}$ \\
\hline Tilt angle & $\gamma$ & $\begin{array}{l}\text { The angle between the surface and horizontal. } \\
\text { South, with east being negative and west being } \\
\text { positive. }\end{array}$ \\
\hline Hour angle & $\omega$ & $\begin{array}{l}\text { The angular displacement of the sun to the east or } \\
\text { west of the local meridian due to rotation of the } \\
\text { earth, with solar noon zero, morning negative and } \\
\text { afternoon positive. }\end{array}$ \\
\hline Zenith angle & $\theta_{z}$ & $\begin{array}{l}\text { The angle between the vertical and the line to the } \\
\text { sun. }\end{array}$ \\
\hline Solar altitude angle & $\alpha_{s}$ & $\begin{array}{l}\text { The angle between the horizontal and the line to the } \\
\text { sun. }\end{array}$ \\
\hline Solar azimuth angle & $\begin{array}{l}\text { The angular displacement from south of the } \\
\text { projection of the beam radiation on a horizontal } \\
\text { surface, with east being negative and west being } \\
\text { positive. }\end{array}$ \\
\hline
\end{tabular}


The first step of the calculation procedure is to calculate the solar time, since all angles are relative to solar noon, and not the local time. Equations 4-1 to 4-3 are used to calculate solar time.

$$
\begin{gathered}
\text { Solar time }=\text { Standard time }+4\left(L_{s t}-L_{l o c}\right)+E \\
E=229.2(0.000075+0.001868 \cos B-0.032077 \sin B-0.014615 \cos 2 B \\
-0.04089 \sin 2 B) \\
B=(n-1) \frac{360}{365}
\end{gathered}
$$

Where $L_{s t}$ is the standard meridian for the time zone, $L_{l o c}$ is the longitude of the location, $E$ is the equation of time in minutes, and $n$ is the day of the year. Knowing the location and time dependent angles, the angle of incidence $(\theta)$ can then be calculated using Equation 4-4.

$$
\begin{gathered}
\cos \theta=\sin \delta \sin \phi \cos \beta-\sin \delta \cos \phi \sin \beta \cos \gamma+\cos \delta \cos \phi \cos \beta \cos \omega \\
+\cos \delta \sin \phi \sin \beta \cos \gamma \cos \omega+\cos \delta \sin \beta \sin \gamma \sin \omega
\end{gathered}
$$

\subsubsection{Incident Solar Radiation}

The solar radiation incident on any given surface (with tilt angle $\beta$ ) is calculated using the isotropic sky model, shown in Equation 4-5.

$$
I_{t o t}=I_{D N I} \cos \theta+I_{d i f f}\left(\frac{1+\cos \beta}{2}\right)+I_{G H I} \rho_{g}\left(\frac{1-\cos \beta}{2}\right)
$$

Where $I_{\text {tot }}$ is the total incident solar radiation on the surface, $I_{D N I}$ is the direct normal beam radiation, $I_{\text {diff }}$ is the diffuse radiation, and $I_{G H I}$ is the global horizontal irradiance, all in W/m $/ \mathrm{m}^{2} . \rho_{g}$ is the ground reflectivity, which was assumed to be 0.2 for all calculations (Enriquez, Zarzalejo, Jiménez, \& Heras, 2012).

\subsubsection{Equivalent Sol-Air Temperature}

The sol-air temperature $\left(T_{s a}\right)$ is an equivalent temperature that would cause the same radiative heat transfer effects in the absence of any radiation. Using the sol-air temperature, the heat transfer 
through wall sections may be calculated using only conductive and convective terms. The sol-air temperature is calculated in Equation 4-6 (Al-Saud, 2009).

$$
T_{s a}=T_{a m b}+\frac{\alpha I}{h_{o}}-\frac{\varepsilon \Delta \mathcal{R}}{h_{o}}
$$

Where $\alpha$ is the absorptance of the surface, $I$ is the total solar radiation incident on the surface $\left(\mathrm{W} / \mathrm{m}^{2}\right), \varepsilon$ is the emissivity of the exterior surface, and $\Delta \mathcal{R}$ is the difference in longwave radiation incident on the surface and the radiation emitted by a black body at the outdoor air temperature $\left(\mathrm{W} / \mathrm{m}^{2}\right)$. The radiation emitted by a black body is calculated in Equation 4-7.

$$
E_{B}=\sigma T_{b}^{4}
$$

$\sigma$ is the Stefan-Boltzmann constant $\left(5.670 \times 10^{-8} \mathrm{~W} / \mathrm{m}^{2} \cdot \mathrm{K}^{4}\right)$, and $T_{b}$ is the temperature of the black body in Kelvin. Once the sol-air temperature has been calculated, it replaces the ambient temperature $\left(T_{a m b}\right)$ in heat flow calculations, accounting for the radiative effects on the heat flow through the walls and windows of the building.

\subsubsection{Convective Heat Transfer Coefficient}

Changes in the convective heat transfer coefficient on the exterior of a building are primarily affected by the air flow, or wind speed, over the surface. Analytical correlations can be derived from heat and momentum transfer principles using dimensionless parameters. For simplicity, empirical correlations, summarized in Equation 4-8, which are commonly used for building applications have been used in this work, (Defraeye, Blocken, \& Carmeliet, 2010).

$$
h_{c}(u)=\left\{\begin{aligned}
4.0 u+5.6, & u<5.0 \mathrm{~m} / \mathrm{s} \\
7.1 u^{0.78}, & u \geq 5.0 \mathrm{~m} / \mathrm{s}
\end{aligned}\right.
$$

Where $h_{c}$ is the convective heat transfer coefficient $\left(\mathrm{W} / \mathrm{m}^{2} \cdot \mathrm{K}\right)$, and $u$ is the wind speed over the surface. For the interior walls, the calculation of $h_{c}$ assumed negligible wind speed, and a value of $5.6 \mathrm{~W} / \mathrm{m}^{2} \cdot \mathrm{K}$ was assumed. 


\subsection{House Model}

A simplified, lumped parameter grey-box house model has been developed for simulation purposes. The model is primarily based on thermodynamic principals, and in-part on the work conducted by other graduate students in this research group (Alibabaei, 2016). The model has been calibrated on both a short- and long-term basis; the former, to capture the relatively slow thermal response of the house, and the latter, to ensure that simulated annual heating and cooling loads are accurate. Data from ASH-A was used for calibration purposes.

The purpose of the model is to quantify the building's zone temperature response to changes in weather, and use this information to determine the resulting heating or cooling load. An energy balance has been performed on all of the wall and window sections of the building. The overall energy flows have been broken down into four main components:

1. Heat flow through the windows $\left(q_{w d}\right)$,

2. Solar heat gain through windows $\left(q_{\text {solar,dir }}\right.$ and $\left.q_{\text {solar,diff }}\right)$,

3. Heat flow through the walls $\left(q_{w}\right)$, and

4. Ventilation heat gains or losses $\left(q_{\text {vent }}\right)$.

Knowing the heat flows into and out of the building, the change in zone temperature can be calculated. Additional heat sources, such as the heat generated by appliances or occupants, have been neglected for this model.

\subsubsection{Heat Flow Through Windows}

The light - and therefore energy - that windows allow into the building has an impact on the heat flows throughout the rest of the building. Therefore, energy balance calculations are first performed on the windows of the building. Figure 4-4 illustrates the simplified window model used in calculations. 


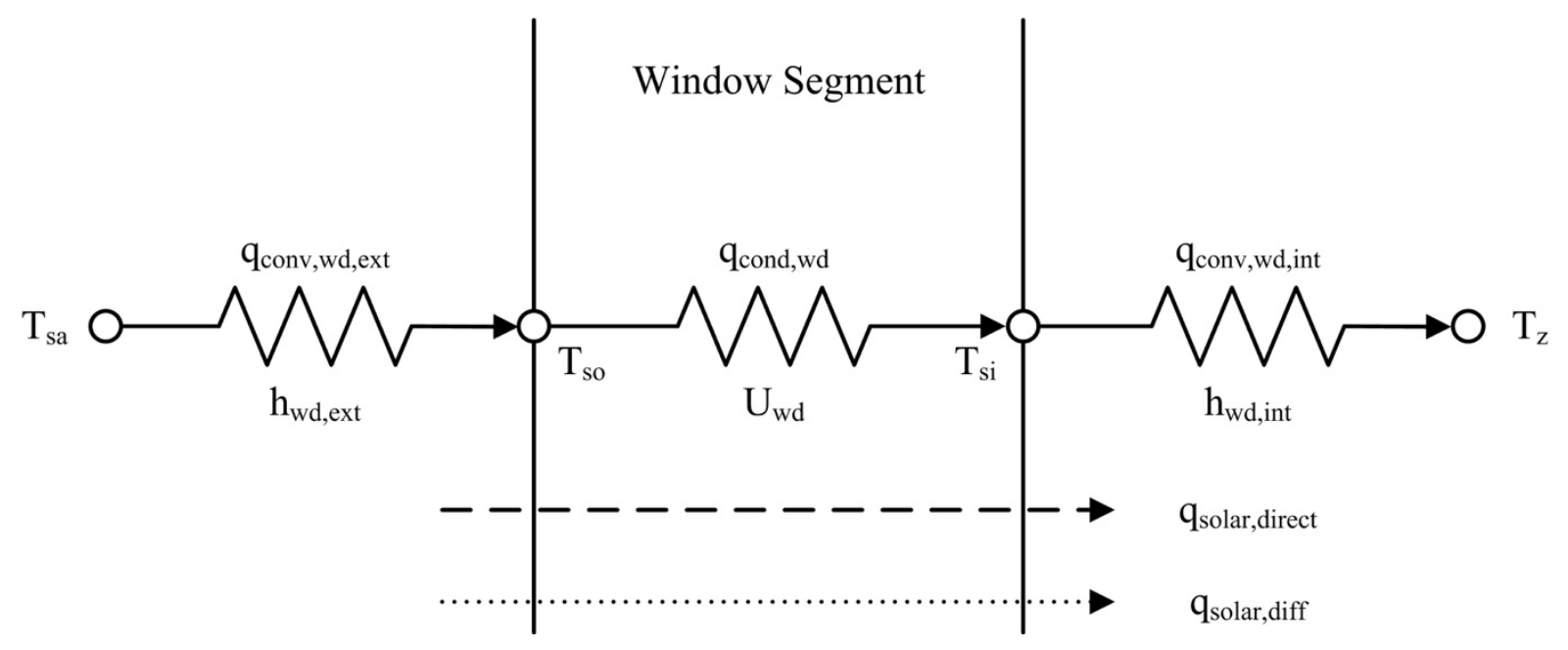

Figure 4-4: Window heat flow diagram

The total heat flow through the windows of the house is calculated in Equations 4-9 and 4-10. Due to their relatively low thermal mass, any heat gain of the windows themselves was neglected. The total conductive and convective resistance of the windows $\left(R_{t o t, w d, i}\right)$ is calculated in Equation $4-11$.

$$
\begin{gathered}
Q_{w d, i}=\frac{T_{s a, i}-T_{z}}{R_{\text {totwd }, i}} \\
Q_{w d, t o t}=\sum_{i=1}^{N_{w d}} Q_{\text {window }, i} \\
R_{t o t, w d, i}=\frac{1}{h_{o} A_{w d, i}}+\frac{1}{U_{w d} A_{w d, i}}+\frac{1}{h_{i} A_{w d, i}}
\end{gathered}
$$

Where $Q_{w d, i}$ is the heat flow through the windows on the $i^{\text {th }}$ side of the house (W) and $Q_{w d, t o t}$ is the total heat flow through all the windows of the house. $N_{w d}$ is the number of windows on the house and $A_{w d, i}$ is the total window area on the $i^{t h}$ side of the house. $h_{o}$ and $h_{i}$ are the exterior and interior convective heat transfer coefficients respectively $\left(\mathrm{W} / \mathrm{m}^{2} \cdot \mathrm{K}\right)$ and $U_{w d}$ is the overall heat transfer coefficient for the window $\left(\mathrm{W} / \mathrm{m}^{2} \cdot \mathrm{K}\right)$, including any air gap in the case of double glazed windows. $h_{o}$ and $h_{i}$ are calculated using the correlation in Equation 4-8, while $U_{w d}$ is obtained 
from the window manufacturer data sheet. The sol-air temperature for the windows is calculated separately from that of the walls, in accordance with the properties of the windows.

The absorptance of the windows is required to calculate the sol-air temperature, and is a function of incidence angle, $\theta$. Once the incident angle of solar irradiance is known, the absorptance of the windows was calculated using the correlations in Equations 4-12 to 4-13 (Parkin, 2015).

$$
\begin{aligned}
\alpha & =1-e^{-k L / \cos \theta_{g l}} \\
\theta_{g l} & =\sin ^{-1}\left(\frac{\eta_{1}}{\eta_{2}} \sin \theta\right)
\end{aligned}
$$

Where $k$ is the extinction coefficient of glass $\left(\mathrm{m}^{-1}\right), L$ is the thickness of the glass $(\mathrm{m})$, and $\theta_{g l}$ is the angle of light within the glass, calculated using Snell's Law (Equation 4-13). $\eta_{1}$ and $\eta_{2}$ are the refractive indexes for air and glass, respectively.

\subsubsection{Solar Heat Gain Through Windows}

The impact of solar heat gains through the windows on the rest of the house is of critical importance, particularly during the cooling season. The solar heat gain through the windows is split into its direct and diffuse (including total horizontal and ground reflected) components. The solar heat gain through the windows is calculated in Equations 4-14 to 4-15 (NFRC, 2006).

$$
\begin{gathered}
q_{\text {solar }, \text { dir }, i}=S H G C_{i} \cdot A_{w d, i} \cdot I_{d i r, i} \\
q_{\text {solar }, \text { diff }, i}=S H G C_{i} \cdot A_{w d, i} \cdot I_{d i f f, i}
\end{gathered}
$$

Where $q_{\text {solar,dir, } i}$ and $q_{\text {solar,diff,i }}$ are the direct and diffuse components of solar heat gain (W), respectively, $S H G C_{i}$ is the solar heat gain coefficient, and $A_{w d, i}$ is the window area of the $i^{\text {th }}$ window. $I_{d i r, i}$ and $I_{d i f f, i}$ are the direct and diffuse incident radiation on the $i^{\text {th }}$ window $\left(\mathrm{W} / \mathrm{m}^{2}\right)$. Typically, separate solar heat gain coefficients would be used for direct and diffuse radiation, both a function of incidence angle. However, for simplicity, a fixed value of 0.56 , obtained from the manufacturer data sheet, was used. 
The solar radiation on all interior surfaces (walls, ceiling, and floor) must be calculated in order to perform energy balances on those surfaces. It has been assumed that all direct transmitted solar radiation first contacts the floor where a portion is absorbed, and a portion is reflected. The reflected portion is assumed to be entirely converted into diffuse radiation. Equation 4-16 calculates the solar radiation on all interior surfaces except the floor, while Equation 4-17 calculates the solar radiation on the floor (McQuiston, Parker, \& Spitler, 2005).

$$
\begin{gathered}
q_{\text {solar }, \text { int }, i}=\frac{\sum q_{\text {solar }, \text { diff }}+\left(1-\alpha_{\text {floor }}\right) \sum q_{\text {solar }, \text { dir }}}{A_{\text {tot }}} \\
q_{\text {solar }, \text { int }, \text { floor }}=\frac{\sum q_{\text {solar }, \text { diff }}+\left(1-\alpha_{\text {floor }}\right) \sum q_{\text {solar }, \text { dir }}}{A_{\text {tot }}}+\frac{\alpha_{\text {floor }} \sum q_{\text {solar }, \text { dir }}}{A_{\text {floor }}}
\end{gathered}
$$

Where $\alpha_{\text {floor }}$ is the absorptance of the floor, $A_{\text {tot }}$ is the total interior surface area $\left(\mathrm{m}^{2}\right)$, and $A_{\text {floor }}$ is the total floor area $\left(\mathrm{m}^{2}\right) . q_{\text {solar,int, } i}$ and $q_{\text {solar,int,floor }}$ are the absorbed solar radiation by the interior walls and ceiling, and floor respectively $\left(\mathrm{W} / \mathrm{m}^{2}\right)$. These values are later used in the inner wall surface energy balance, described in the following section.

\subsubsection{Heat Flow Through Walls}

The wall segments are modeled as lumped resistive-capacitive thermal networks. The lumped capacitive method is a simple method for performing heating and cooling calculations. Typically, the validity of this method for a given case is tested using the Biot number $(B i)$, a dimensionless parameter that represents the ratio of convective resistance to conductive resistance. The Biot number is defined in Equation 4-18. (Bergman, Lavine, Incropera, \& Dewitt, 2011).

$$
B i=\left(\frac{h L}{k}\right)
$$

When $B i<0.1$, the conductive resistance is much less than the convective resistance, indicating a relatively uniform temperature within the solid. Typically, the lumped capacitance method is only valid when $B i<0.1$. 
In the case of the walls used in the house model, $0.1<B i<100$, which falls outside the typical allowable range. However, research has found that validity of the lumped capacitance method can be extended to larger Biot numbers, up to 100. This research was conducted for building-integrated thermal storage applications, which requires highly-accurate thermal models. It has been found that the lumped capacitance method is valid for Biot numbers as large as $100(\mathrm{Xu}, \mathrm{Li}, \&$ Chan, 2012). The greatest accuracy was reported when the system had a smaller dimensionless time value, $\tau$. For this system, $\tau=0.00014$, which is sufficiently small and indicates that the lumped capacitance model is an acceptable approach.

Figure 4-5 displays the simplified wall model used to determine the heat flow through the walls of the house. Energy balances around the three wall nodes for the $i^{\text {th }}$ wall section are shown in Equations 4-19 through 4-21.

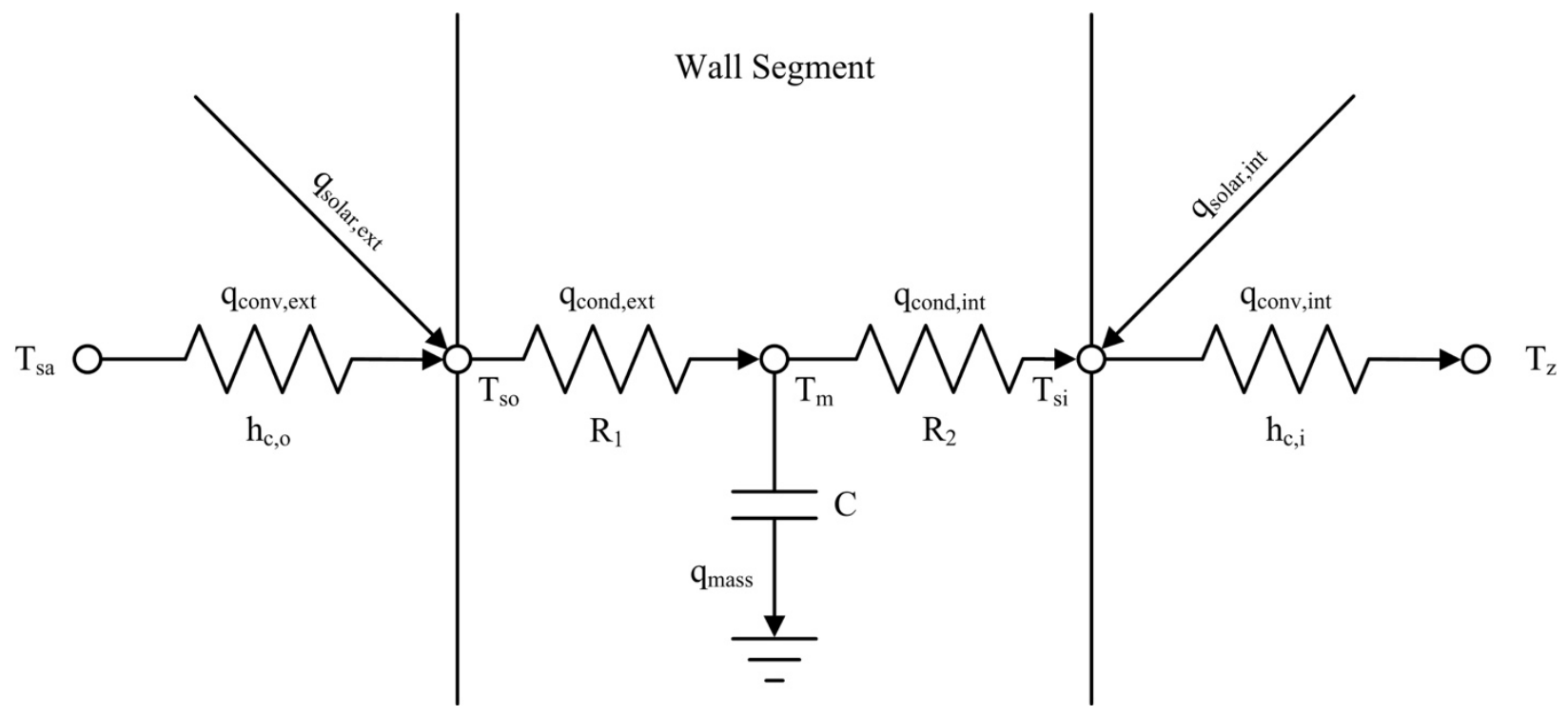

Figure 4-5: Wall heat flow diagram

$$
\begin{gathered}
q_{\text {solar }, \text { ext }, i}+q_{\text {conv }, \text { ext }, i}+q_{\text {rad }, \text { ext }, i}=q_{\text {cond }, \text { ext }, i} \\
C_{i} \frac{d T_{m, i}}{d t}=q_{\text {cond }, \text { ext }, i}-q_{\text {cond }, \text { int }, i} \\
q_{\text {cond }, \text { int }, i}+q_{\text {solar }, \text { int }, i}=q_{\text {conv }, \text { int }, i}
\end{gathered}
$$


Where the subscripts ext and int indicate the exterior and interior of the building respectively, and cond and conv refer to conductive and convective heat flows respectively. All heat flows are in watts. Equations 4-22 through 4-25 define the conductive and convective heat flows through the wall section. Note that because Equation 4-22 uses the sol-air temperature, $q_{\text {conv }, \text { ext }, i}$ accounts for both convective and radiative heat flows.

$$
\begin{gathered}
q_{c o n v, e x t, i}=h_{c, o} A_{w, i}\left(T_{s a, i}-T_{s o, i}\right) \\
q_{c o n d, e x t, i}=\frac{\left(T_{s o, i}-T_{m, i}\right)}{R_{1}} \\
q_{c o n v, i n t, i}=h_{c, i} A_{w, i}\left(T_{s i, i}-T_{z}\right) \\
q_{c o n d, e x t, i}=\frac{\left(T_{m, i}-T_{s i, i}\right)}{R_{2}}
\end{gathered}
$$

$T_{s o, i}$ and $T_{s i, i}$ are the exterior and interior wall surface temperatures respectively $\left({ }^{\circ} \mathrm{C}\right)$. At this point in the calculation, neither of these surface temperatures are known, but are required in order to calculate the change in thermal mass temperature at the following time step. Equation 4-26 is used to calculate the exterior surface temperature, while Equation 4-27 is used to calculate the interior wall surface temperature.

$$
\begin{gathered}
h_{c, o} A_{w, i}\left(T_{s a, i}-T_{s o, i}\right)=\frac{\left(T_{s o, i}-T_{m, i}\right)}{R_{1}} \\
\frac{\left(T_{m, i}-T_{s i, i}\right)}{R_{2}}+q_{s o l a r, i n t, i}=h_{c, i} A_{w, i}\left(T_{s i, i}-T_{z}\right)
\end{gathered}
$$

Once the two wall surface temperatures are known, the interior convective heat flows are summed, and used in the zone air temperature balance, covered in Section 4.4.6. The change in thermal mass temperature, for use in the following time step, is calculated in Equations 4-28 and 4-29 using a numerical method based on the stability criterion $(\tau)$ (Çengel \& Ghajar, 2010). These equations are derived from the energy balance at the middle node in Figure 4-5. Where $d x$ is the half-wall length, and $\tau$ is the dimensionless time, also known as the Fourier number, Fo. 


$$
\begin{gathered}
\tau=\frac{k}{\rho c_{p}} \frac{d t}{d x^{2}} \\
T_{m, i}{ }^{t+1}=\tau\left(T_{s o, i}{ }^{t}+T_{s i, i}{ }^{t}\right)+(1-2 \tau) T_{m, i}{ }^{t}
\end{gathered}
$$

\subsubsection{Ventilation Heat Flow}

The house model accounts for both natural and forced ventilation. The details of forced ventilation are described with respect to HRV operation in Section 4.5.1. Natural ventilation - Infiltration is the leakage of air into or out of a building. Heat gains or losses can be attributed to infiltration due to differences in indoor and outdoor air temperature. The air change method has been used to calculate infiltration. Equation 4-30 is used to calculate the volumetric flow rate of infiltration air (Howell, Coad, \& Sauer, 2013).

$$
\dot{Q}=A C H_{\text {avg }} V_{z} \frac{1}{3600}
$$

Where $\dot{Q}$ is the volumetric flow rate of air $\left(\mathrm{m}^{3} / \mathrm{s}\right), A C H_{\text {avg }}$ is the average air changes per hour of the house (ach), and $V_{z}$ is the total zone volume $\left(\mathrm{m}^{3}\right)$. The average air changes per hour are computed using $\mathrm{ACH}_{50}$, the air change at $50 \mathrm{~Pa}$, described in Equation 4-31 (Zhang, Fung, \& Jhingan, 2014).

$$
A C H_{\text {avg }}=\frac{A C H_{50}}{N_{0} H_{1} S_{2} L_{3}}
$$

Where $N_{0}$ is the leakage-infiltration ratio, $H_{1}$ is the building height correction factor, $S_{2}$ is the site shielding factor, and $L_{3}$ is the leakiness corretion factor. These four factors are all climate- and building-specific. For Toronto, Canada, $N_{0}$ can be taken to be 19 (Zhang, Fung, \& Jhingan, 2014). Typical values for all other parameters in Equation 4-31 are shown in Table 4-7 and Table 4-8. 
Table 4-7. Typical air change rates for different building types (Zhang, Fung, \& Jhingan, 2014)

\begin{tabular}{|l|c|}
\hline \multicolumn{1}{|c|}{ House Type } & Air change at 50 Pa $-\boldsymbol{A C H}_{\mathbf{5 0}}$ \\
\hline Loose & 10.35 \\
\hline Average & 4.55 \\
\hline Present & 3.57 \\
\hline Energy-Efficient & 1.5 \\
\hline Archetype House A & 1.204 (Fung, Dembo, \& Zhou, 2009) \\
\hline
\end{tabular}

Table 4-8. Typical infiltration correction factor values (Sherman, 1986)

\begin{tabular}{|l|c|}
\hline Number of Stories & Height correction factor $-\boldsymbol{H}_{\mathbf{1}}$ \\
\hline 1 & 1 \\
\hline 1.5 & 0.9 \\
\hline 2 & 0.8 \\
\hline 3 & 0.7 \\
\hline Condition & Shielding correction factor $-\boldsymbol{S}_{\mathbf{2}}$ \\
\hline Well Shielded & 1.2 \\
\hline Normal & 1.0 \\
\hline Exposed & 0.9 \\
\hline Condition & Leakiness correction factor $-\boldsymbol{L}_{\mathbf{3}}$ \\
\hline Small cracks (tight) & 1.4 \\
\hline Normal & 1.0 \\
\hline Large holes (loose) & 0.7 \\
\hline
\end{tabular}

Once the volumetric flow rate of air is known, the sensible heating or cooling from infiltration $\left(q_{\text {inf,sens }}\right)$ is calculated using Equation 4-32. The same method for calculating the infiltration heat flow can be used to calculate the load associated with the HRV, and the total ventilation heat flow is calculated in Equation 4-33.

$$
\begin{gathered}
q_{i n f, \text { sens }}=\dot{Q} \rho_{\text {air }} c_{p, a i r}\left(T_{a m b}-T_{z}\right) \\
q_{v e n t, \text { sens }}=q_{i n f, \text { sens }}+q_{H R V, \text { sens }}
\end{gathered}
$$

\subsubsection{Latent Cooling}

A latent heating or cooling process causes a change in humidity ratio of air, but not temperature. Along with temperature, humidity plays a significant role in the thermal comfort of a space. Figure 4-6 displays ASHRAE summer and winter thermal comfort zones based on temperature and 
humidity. Since there is no recommended lower humidity limit, only an upper limit on humidity ratio has been applied to this model. Therefore, the model developed only considers the impact of latent cooling on the house, and not latent heating.

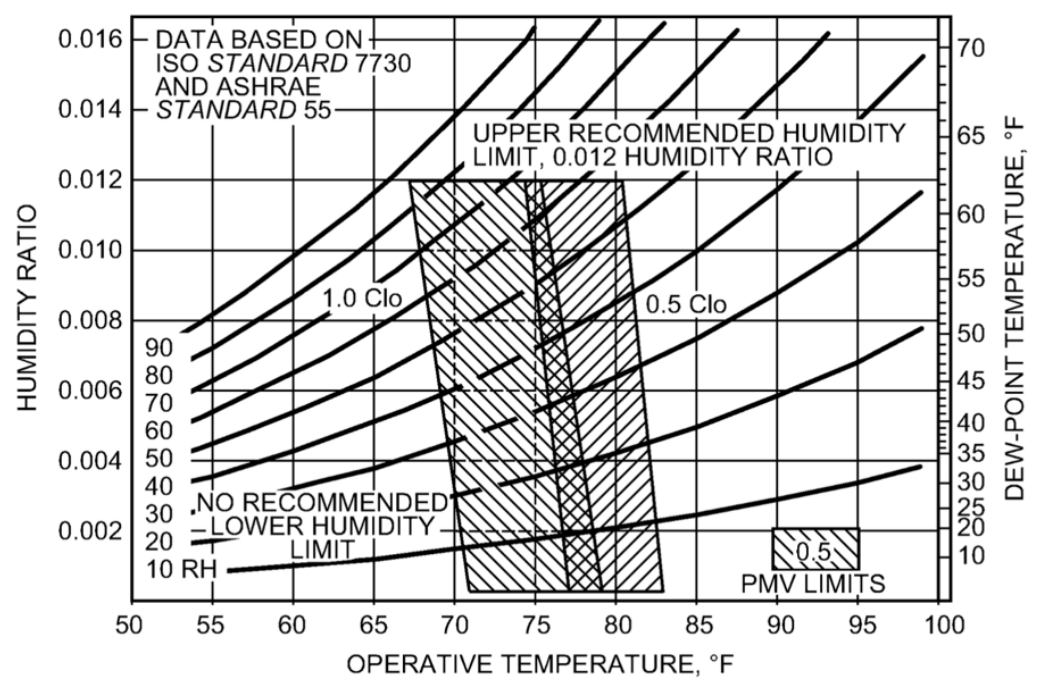

Figure 4-6: ASHRAE summer and winter thermal comfort zones (Howell, Coad, \& Sauer, 2013)

The change in humidity ratio is assumed to arise entirely from ventilation air into the zone. Similar to Equation 4-32, the latent heat due to ventilation (both forced and natural) is calculated in Equation 4-34.

$$
q_{v e n t, l a t}=\dot{Q} \rho_{a i r} h_{f g}\left(w_{o}-w_{z}\right)
$$

Where $h_{f g}$ is the specific enthalpy of evaporation of water $(2256.7 \mathrm{~kJ} / \mathrm{kg})$, and $w_{o}$ and $w_{z}$ are the humidity ratios of the outdoor and zone air respectively ( $\mathrm{kg}$ water $/ \mathrm{kg}$ dry air). This additional cooling load is only calculated when the zone air humidity ratio rises above 0.012 the upper limit based on ASHRAE thermal comfort standards. For this model, all of the energy required for latent cooling is assumed to be provided by the ASHP. 


\subsubsection{Zone Air Energy Balance}

Once the heat loss of gain from the walls, windows, and infiltration have been calculated, the change in zone air temperature can be determined. The energy balance on the air for the singlezone house is displayed in Equation 4-35

$$
C_{\text {air }} \frac{d T_{z}}{d t}=\sum_{i=1}^{N_{w}} q_{c o n v, \text { int }, w, i}+\sum_{i=1}^{N_{w d}} q_{c o n v, \text { int }, w d, i}+q_{v e n t, \text { sens }}+q_{v e n t, l a t}+q_{\text {sup }}
$$

Where $N_{w}$ is the number of walls in the zone, and $N_{w d}$ is the number of windows in the zone, and $q_{\text {sup }}$ is the supplied heat by the HVAC system. $C_{\text {air }}$ is the thermal capacitance of air, defined in Equation 4-36

$$
C_{\text {air }}=\rho_{\text {air }} V_{\text {zone }} C_{p, a i r}
$$

The internal thermal mass of the building (furniture, appliances, etc.) must also be considered. For simplicity, the zone was assumed to have a homogenous temperature distribution. To account for these additional building internal components, $c_{p, \text { air }}$ was replaced with an equivalent heat capacity of the zone $-c_{z, e q}$. This parameter was calibrated to match experimental data along with the other building parameters listed in Table 4-4.

\subsection{Mechanical System Model}

The HVAC model used in this work is comprised of an ASHP, an HRV, and an AHU. These are the three primary mechanical devices utilized in House A. All HVAC model components are based on experimental data.

Figure 4-7 displays a representative example of the power consumption of the three primary mechanical systems in House A. For this model, it was assumed that the HRV had a constant ventilation rate. The multi-speed AHU fan ran on high speed when there was a call for heating or cooling, and low speed otherwise. 


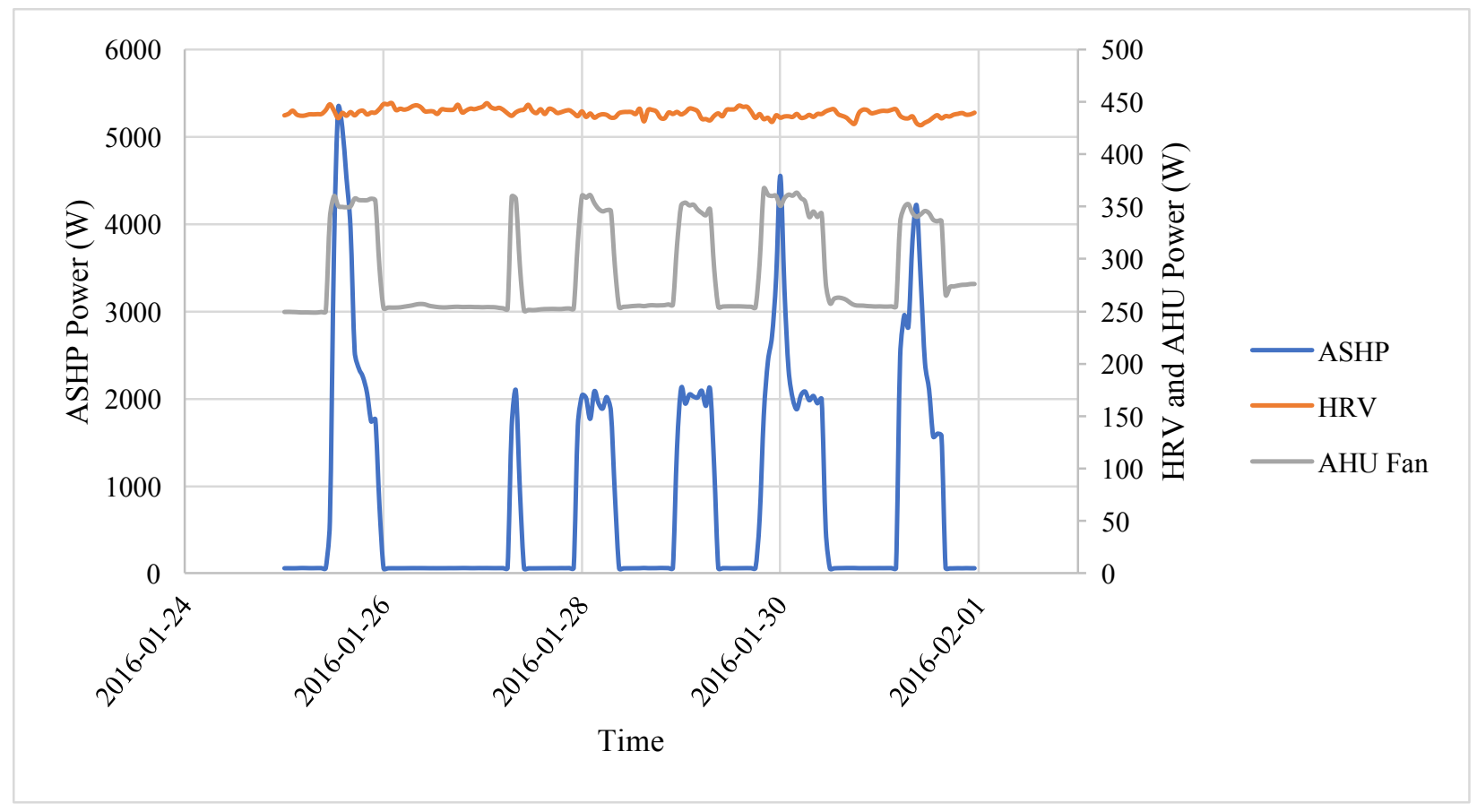

Figure 4-7: House A mechanical system power consumption

\subsubsection{Heat Recovery Ventilator Model}

House A uses a HRV to achieve energy savings when ventilating stale air. The HRV installed in the house is a vänEE $3000 \mathrm{HE}$, with a rated power of $500 \mathrm{~W}$. A model of this HRV was required in order to quantify both the power consumed by the HRV, and the temperature of the air supplied to the zone by the HRV. Experimental data collected over 2016 was used to develop a model for use in this work.

Based on measured data at the ASH, the HRV has been shown to have a stable average power consumption of $432 \mathrm{~W}$. Measurements over 2016 also show a stable, average ventilation rate of $101.7 \mathrm{cfm}\left(0.048 \mathrm{~m}^{3} / \mathrm{s}\right)$. Therefore, fixed HRV ventilation rate and power consumption values have been used for all simulations. Flow measurements were recorded with a Dwyer STRA duct mounted airflow measurement station, with an accuracy of $2 \%$.

An ANN was used to model the heat exchange process occurring in the HRV. The ANN used fresh outdoor air temperature and exhaust zone temperature as inputs. The output of the model was the temperature of the air supplied to the zone by the HRV. The ANN was trained on annual hourly 
data from 2016. When used in simulations, the HRV model uses the ambient temperature from the weather data and the calculated zone temperature as its two inputs. It outputs an air temperature used for forced ventilation heat flow calculations.

An analysis of the performance of the HRV ANN was performed based on hidden layer size. The ANN was trained on the 2016 data set, varying the number of neurons from 1 to 20 , and always using a single hidden layer. Training was performed in MATLAB using the Levenberg-Marquardt algorithm with $70 \%$ of the data used for training, $15 \%$ used for testing, and $15 \%$ used for validation, distributed randomly. This training method was used for all other ANNs in this work unless otherwise specified.

Thirty training iterations were performed for each hidden layer neuron size to generate confident results. The root-mean-square error (RMSE) and the coefficient of determination $\left(\mathrm{R}^{2}\right)$ were both used as metrics to judge the performance of the ANN. Figure 4-8 plots the RMSE and $\mathrm{R}^{2}$ against the number of neurons in the ANN, where the error bars represent the standard deviation of the 30 training trials at each hidden layer size.

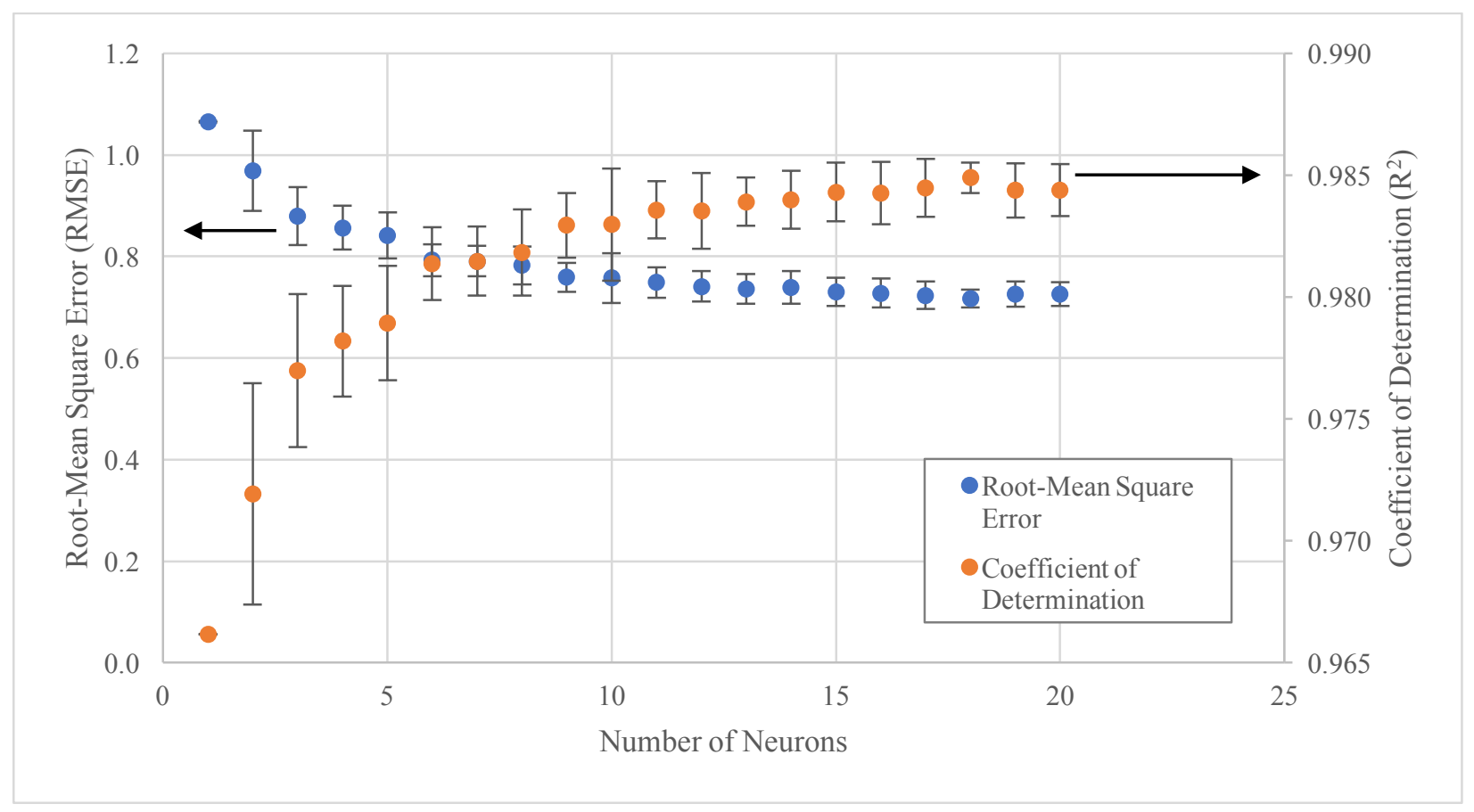

Figure 4-8: Heat recovery ventilator ANN performance 
The final HRV model consisted of 18 neurons in a single hidden layer - the simplest network size that can achieve sufficient accuracy (RMSE $=0.716, \mathrm{R}^{2}=0.985$ ).

\subsubsection{Air Handling Unit Model}

Heating and cooling is distributed throughout the house via the single-zoned AHU. The ASHP has a direct expansion coil in the AHU, and control over the two-speed distribution fan. This is illustrated in Figure 4-7, where it can be clearly seen that the AHU and ASHP operate in synchrony. As such, the AHU model was simply assumed to consume a constant amount of lowspeed power when not in operation $(266 \mathrm{~W})$, and a constant amount of power when a heating or cooling call is made (336W). These values are based on 2016 averages recorded by the DMS.

\subsubsection{Air Source Heat Pump Model}

The simplified ASHP model used in simulations is based on experimental work previously conducted at the ASH (Safa, Fung, \& Kumar, 2015). The heat pump model has been broken down into two components: heating and cooling modes. Table 4-9 highlights the technical specifications of the ASHP installed in House A.

Table 4-9: ASHP technical specifications (Safa, Fung, \& Kumar, 2015)

\begin{tabular}{|c|c|c|l|}
\hline Mode & Rated Capacity & Rated COP & \multicolumn{1}{c|}{ Rated Conditions } \\
\hline Heating & $11.06 \mathrm{~kW}$ & 3.27 & $\begin{array}{l}\text { Indoor: } 21.1^{\circ} \mathrm{C} \text { dry-bulb, } 15.6^{\circ} \mathrm{C} \text { wet-bulb } \\
\text { Outdoor: } 8.3^{\circ} \mathrm{C} \text { dry-bulb, } 6.1^{\circ} \mathrm{C} \text { wet-bulb }\end{array}$ \\
\hline Cooling & $9.82 \mathrm{~kW}$ & 3.52 & $\begin{array}{l}\text { Indoor: } 26.7^{\circ} \mathrm{C} \text { dry-bulb, } 19.4^{\circ} \mathrm{C} \text { wet-bulb } \\
\text { Outdoor: } 35.0^{\circ} \mathrm{C} \text { dry-bulb, } 23.9^{\circ} \mathrm{C} \text { wet-bulb }\end{array}$ \\
\hline
\end{tabular}

\subsubsection{Heating Mode}

The ASHP modeled in this work is a two-stage variable capacity cold climate Mitsubishi Zuba. This heat pump has an internal controller responsible for optimizing the power consumption of the heat pump by modifying the variable-drive compressor. The internal algorithms used by the heat pump are proprietary, and the mechanisms by which the heat pump controls its capacity ratio are unknown. Therefore, the internal details of the heat pump are not captured by this model. Instead, a simplified model has been developed based on experimental operating conditions. 
Figure 4-9 displays the power consumption of the ASHP based on outdoor air temperature. At temperatures below $-15^{\circ} \mathrm{C}$, second stage operation begins and power consumption dramatically increases. The correlations shown in Figure 4-9 have been used to determine the ASHP power consumption in heating mode based on outdoor air temperature. For this model, the effects of indoor air temperature on the heat pump's operation have been ignored.

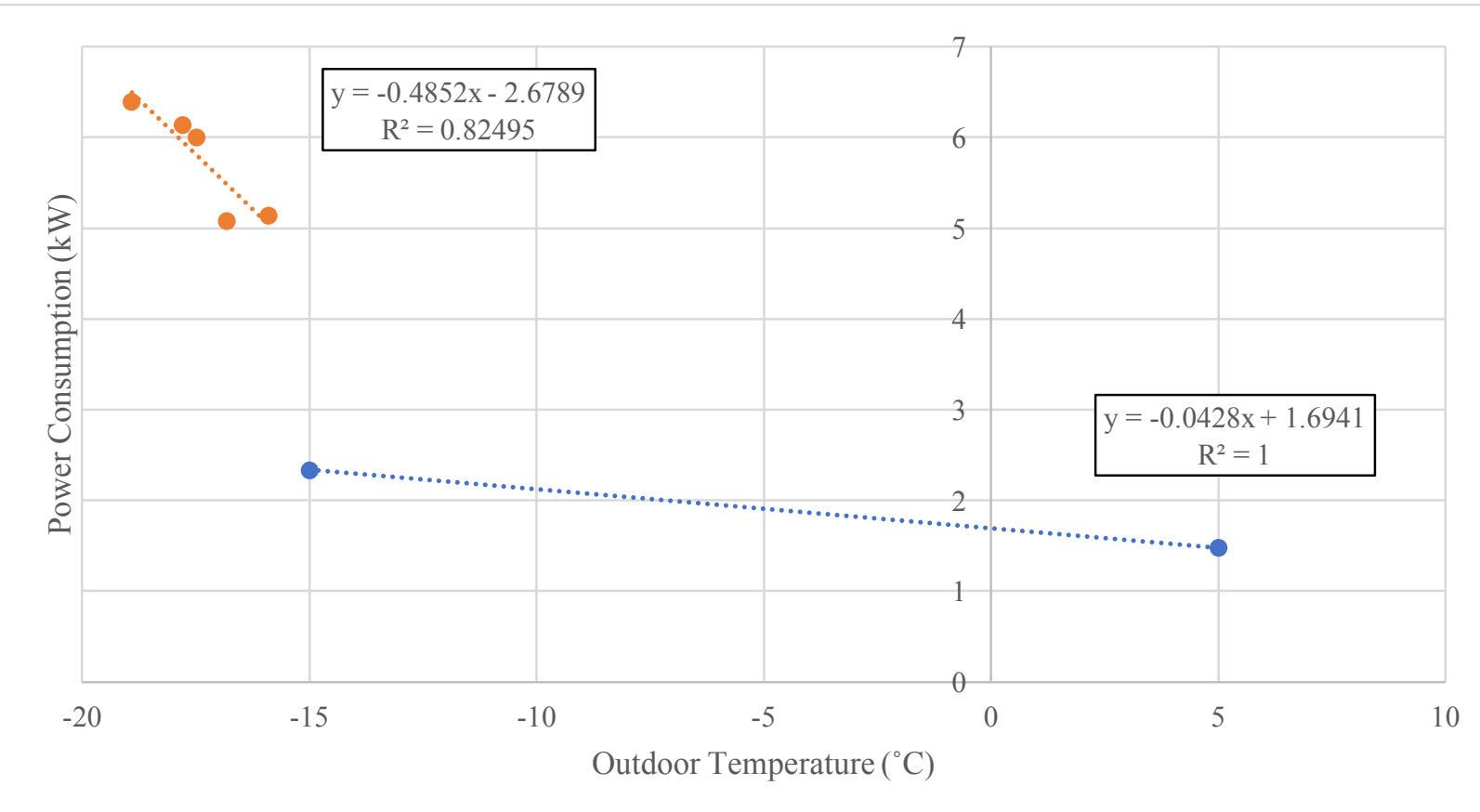

Figure 4-9. ASHP experimental power consumption in both first (right) and second stages (left) (Safa, Fung, \& Kumar, 2015)

Figure 4-10 displays the experimentally determined COP of the ASHP in heating mode. In heating mode, the COP of a heat pump is calculated in Equation 4-37 where $Q_{H}$ is the heat delivered and $W_{\text {elec }}$ is the work input, or power consumption.

$$
C O P_{\text {heating }}=\frac{Q_{H}}{W_{\text {elec }}}
$$




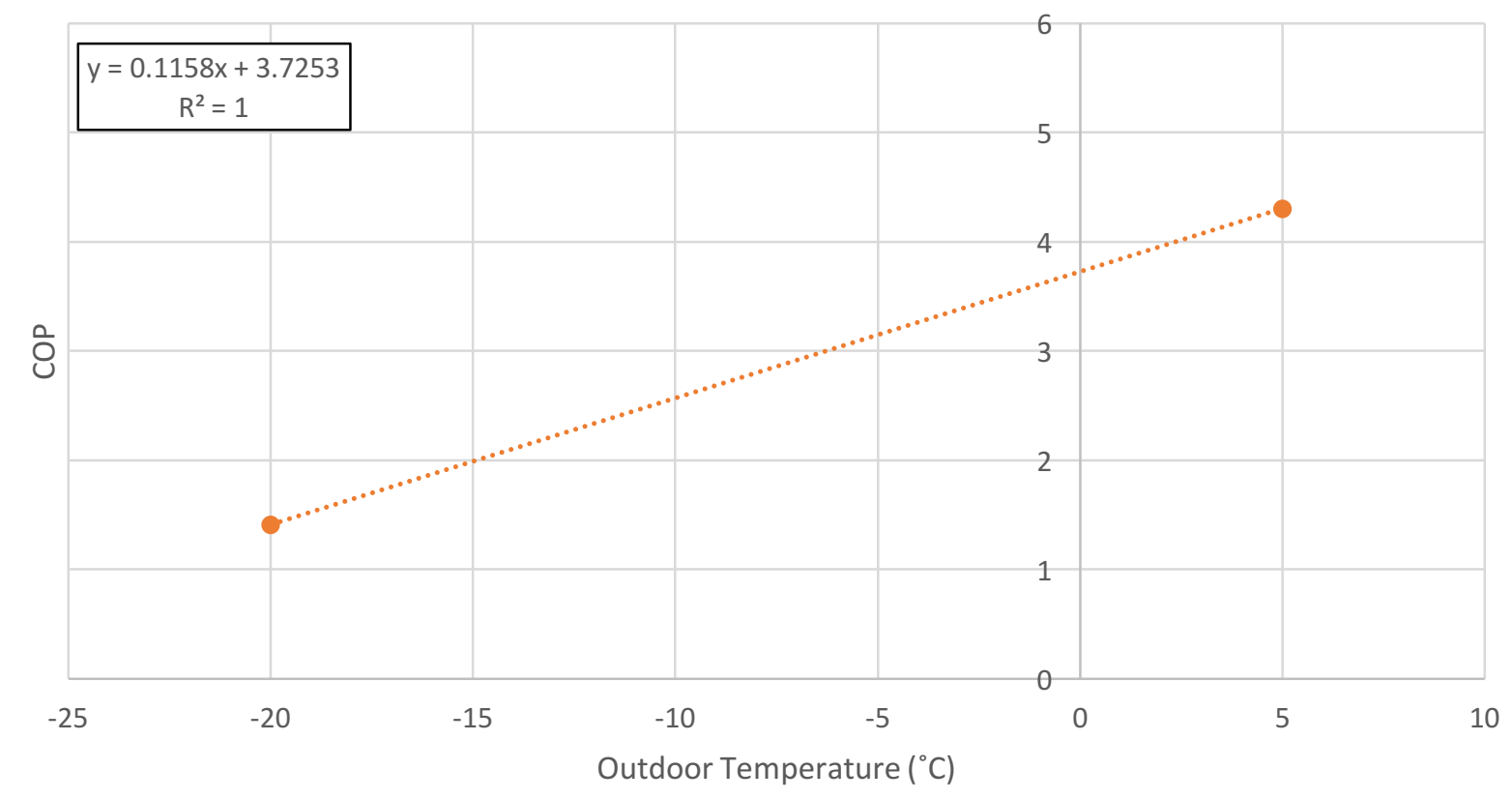

Figure 4-10: ASHP experimental COP as a function of outdoor temperature (Safa, Fung, \& Kumar, 2015)

Extracting the experimental correlations shown in Figure 4-9 and Figure 4-10, the heat delivered to the zone is calculated using Equations 4-38 through 4-40.

$$
\begin{gathered}
W_{\text {elec }}\left(T_{a m b}\right)= \begin{cases}-0.4852 T_{a m b}-2.6789, & T_{a m b}<-15^{\circ} \mathrm{C} \\
-0.0428 T_{a m b}+1.6941, & T_{a m b} \geq-15^{\circ} \mathrm{C}\end{cases} \\
\operatorname{COP}_{\text {heating }}\left(T_{a m b}\right)=0.1158 T_{a m b}+3.7253 \\
q_{\text {sup }}=Q_{H}=C O P_{\text {heating }} W_{\text {elec }}
\end{gathered}
$$

\subsubsection{Cooling Mode}

In cooling mode, the variable-capacity ASHP has a single stage of operation. Again, its internal control algorithm is unknown, and assumptions have been made in order to model the heat pump's operation simply as a function of outdoor temperature. Figure 4-11 displays the reported experimental COP of the ASHP in cooling mode (Safa, Fung, \& Kumar, 2015). For a heat pump operating in cooling mode, the COP is calculated in Equation 4-41, where $Q_{c}$ is the cooling provided by the heat pump. 


$$
\text { COP }{ }_{\text {cooling }}=\frac{Q_{C}}{W_{\text {elec }}}
$$

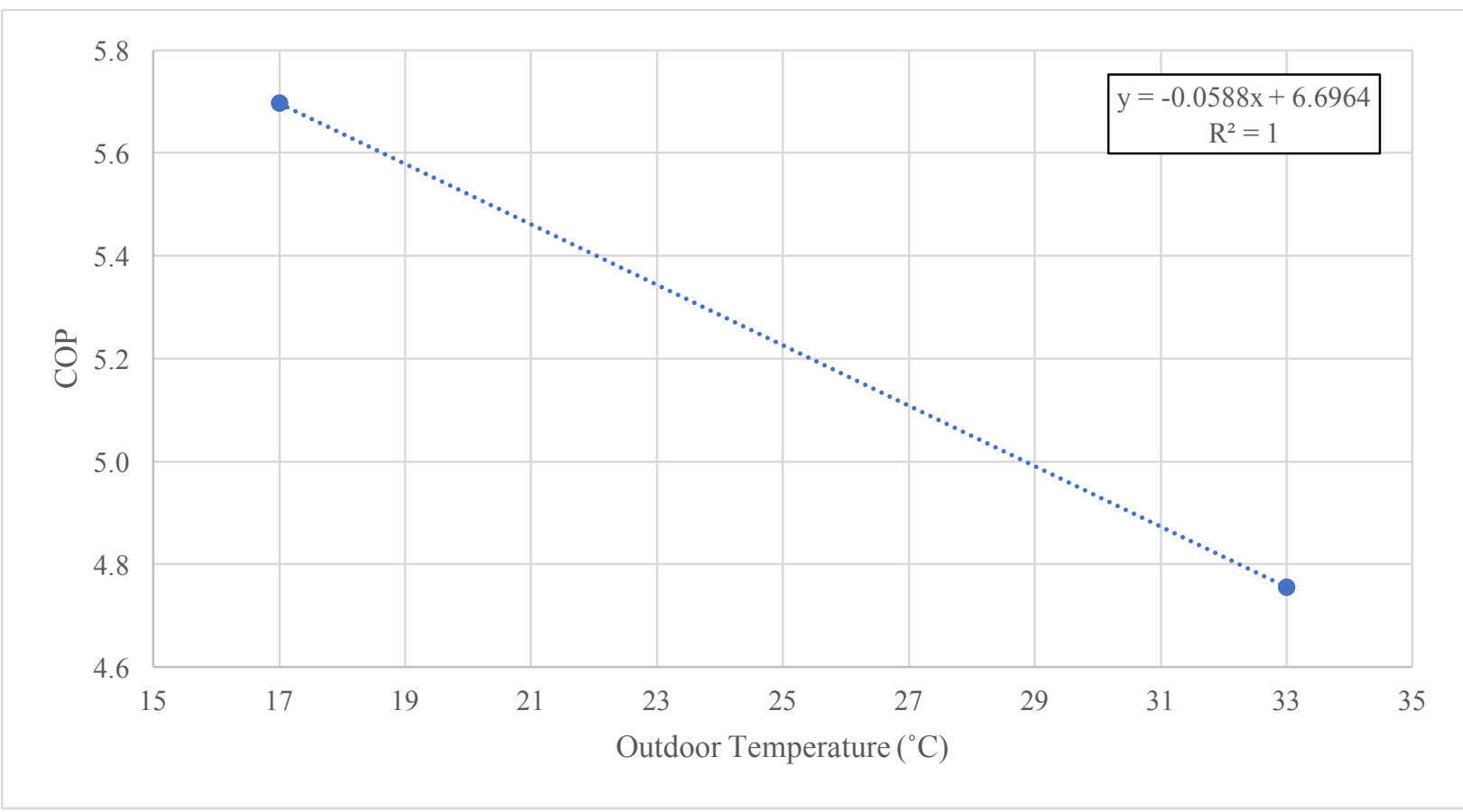

Figure 4-11: COP in cooling mode as a function of outdoor temperature (Safa, Fung, \& Kumar, 2015)

For cooling mode, neither power consumption (as displayed in Figure 4-9), nor cooling output of the heat pump has been provided. However, during cooling mode, the capacity ratio of the heat pump was reported to range between $52-57 \%$ (Safa, Fung, \& Kumar, 2015). The capacity ratio is the percent of the rated capacity of the heat pump at a given temperature condition. It has been assumed that the experimentally reported capacity ratio is equivalent to $5.1-5.6 \mathrm{~kW}$, based on the rated capacity of the heat pump in cooling mode. It was also assumed that the upper and lower ends of the reported capacity ratios were associated with the upper and lower temperature conditions at which the heat pump was monitored. Figure 4-12 summarizes these assumptions, and provides a correlation for the cooling provided by the heat pump $\left(Q_{c}\right)$ in terms of outdoor temperature. The calculation procedure for the heat pump model in cooling mode is summarized in Equations 4-42 through 4-44. 


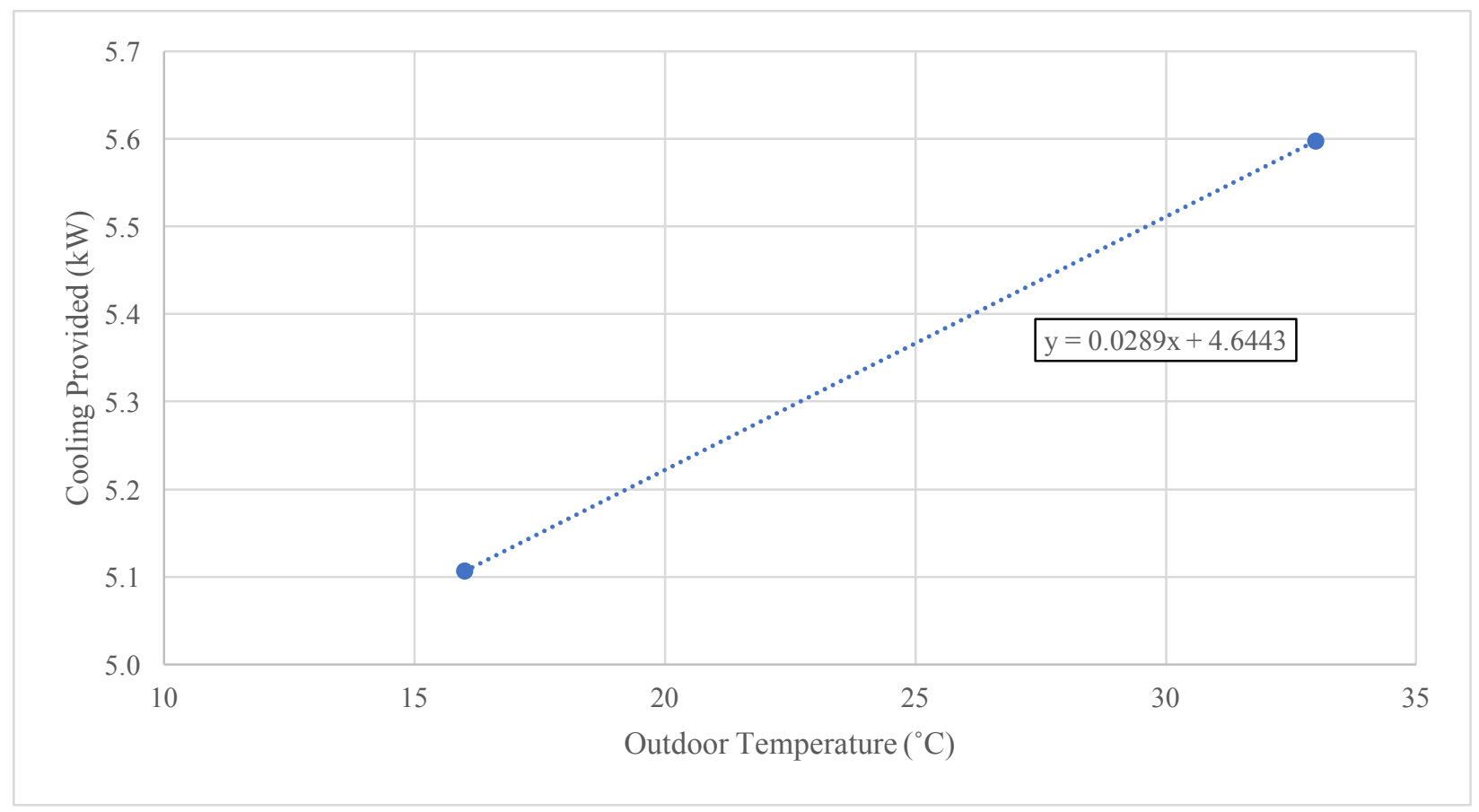

Figure 4-12: ASHP cooling provided as a function of outdoor temperature

$$
\begin{gathered}
Q_{C}\left(T_{a m b}\right)=0.0289 T_{a m b}+4.6443 \\
\operatorname{COP}_{\text {cooling }}\left(T_{a m b}\right)=-0.0588 T_{a m b}+6.6964 \\
W_{\text {elec }}=\frac{Q_{C}}{C O P_{\text {cooling }}}
\end{gathered}
$$

\subsection{House Model Calibration}

Once the structure of the model was complete, the parameters listed as "Calibrated" in Table 4-4 were optimized such that the model matched real-world operation as best as possible. Two separate calibration functions were optimized in parallel, in order to accurately capture both the model's short-term response to weather conditions, along with its long-term (annual) energy consumption.

The short-term calibration was performed using data obtained from a "cool-down test". In early April 2017, the mechanical systems in ASH-A were turned off over the course of a weekend. This allowed the house temperature's natural rise and fall to be monitored. The average ambient temperature over the course of the 60 -hour cool-down test was $5.4^{\circ} \mathrm{C}$. Figure $4-13$ shows the 
temperature and solar irradiance over this test period. A one-minute time step size was used for the short-term calibration. The goal of the short-term calibration was so that the model could properly capture the thermal response speed of the real-world house.

For the short-term calibration, the RMSE between simulated and actual data was used as the performance metric to minimize. The optimization function evaluated the RMSE starting at minute 500 , to the end of the simulation. Neglecting the first 500 minutes of the simulation minimized any error associated with selecting incorrect initial simulation conditions. Since there is no measurement of the thermal mass temperature at the ASH-A this parameter had to be estimated, which primarily impacted the beginning of the simulation.

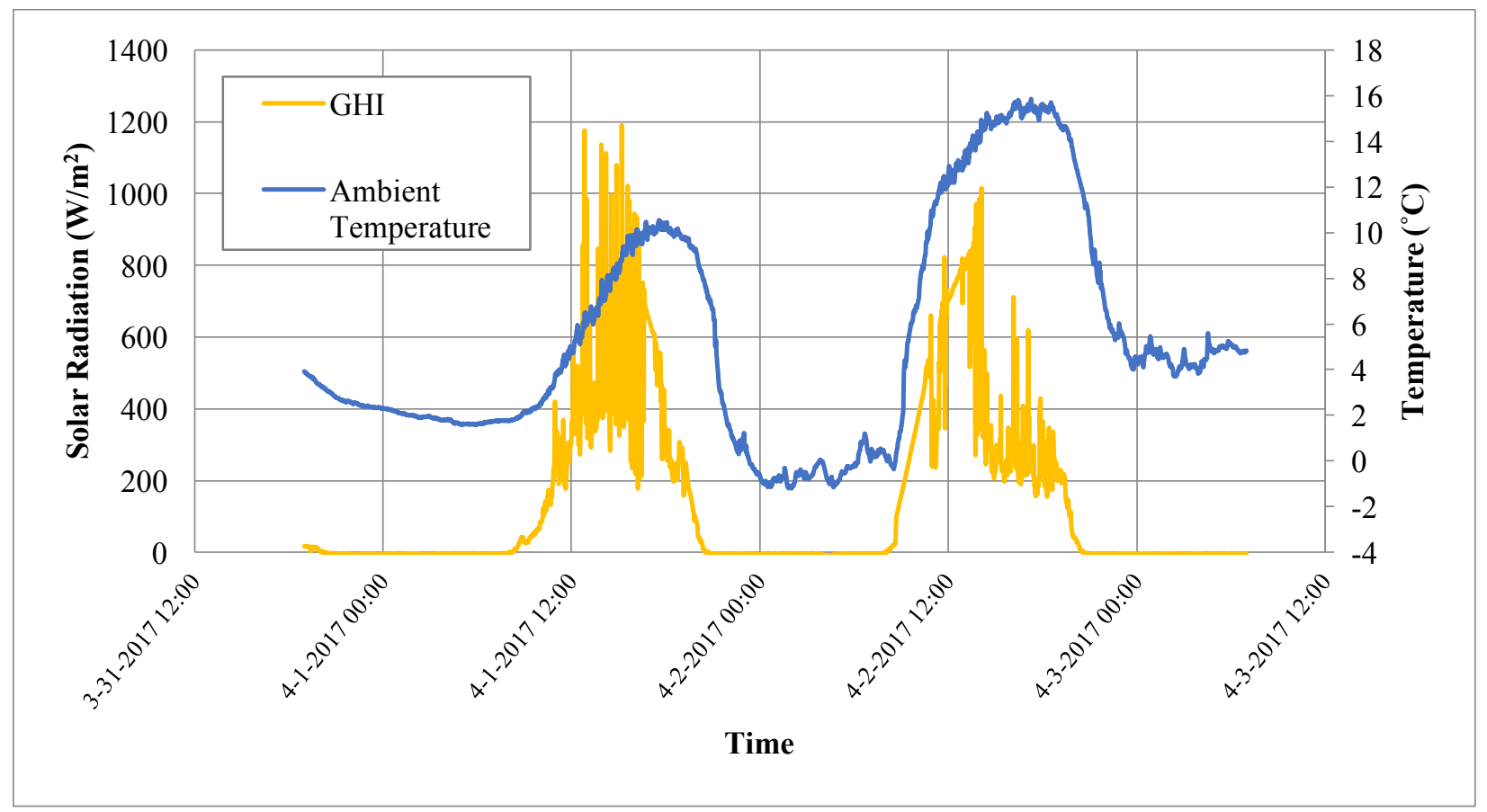

Figure 4-13: Ambient conditions over cool-down test period

The long-term calibration used the annual weather dataset obtained from the Living City Campus averaged at one-hour time intervals. This simulation assumed fixed thermostat set-points of $22^{\circ} \mathrm{C}$ in the heating season and $24^{\circ} \mathrm{C}$ in the cooling season. The goal of this calibration procedure was to minimize the difference between simulated and actual annual heating and cooling energy consumption. The long-term calibration used the ASHP model as the heating and cooling source for the building. The consumption targets were based on the heating or cooling energy delivered 
from TRNSYS models of the ASH-A (Safa, Fung, \& Kumar, 2015). These targets, and the calibrated simulation output, are summarized in Table 4-10. The calibration function used output the percent difference between simulated and actual heating and cooling outputs, which were used as the performance metrics to minimize.

Both the short- and long-term simulations were run in parallel, and the MATLAB function "fmincon" was used to search for the house parameters that achieved the best-fit for both modes of operation simultaneously.

Figure 4-14 and Figure 4-15 show the summary of both the short- and long-term calibration procedures. The short-term model accurately reflects real-world operating conditions, with a RMSE $=0.31^{\circ} \mathrm{C}$, equivalent to a $1.9 \%$ error based on the average temperature over this period. The maximum simulated temperature deviation from the actual temperature over this period was $0.66^{\circ} \mathrm{C}$. The long-term calibration also produced a working model that accurately represents the annual heating and cooling energy consumption of the ASH-A. Table 4-10 summarizes the simulated and target energy consumption of the model.

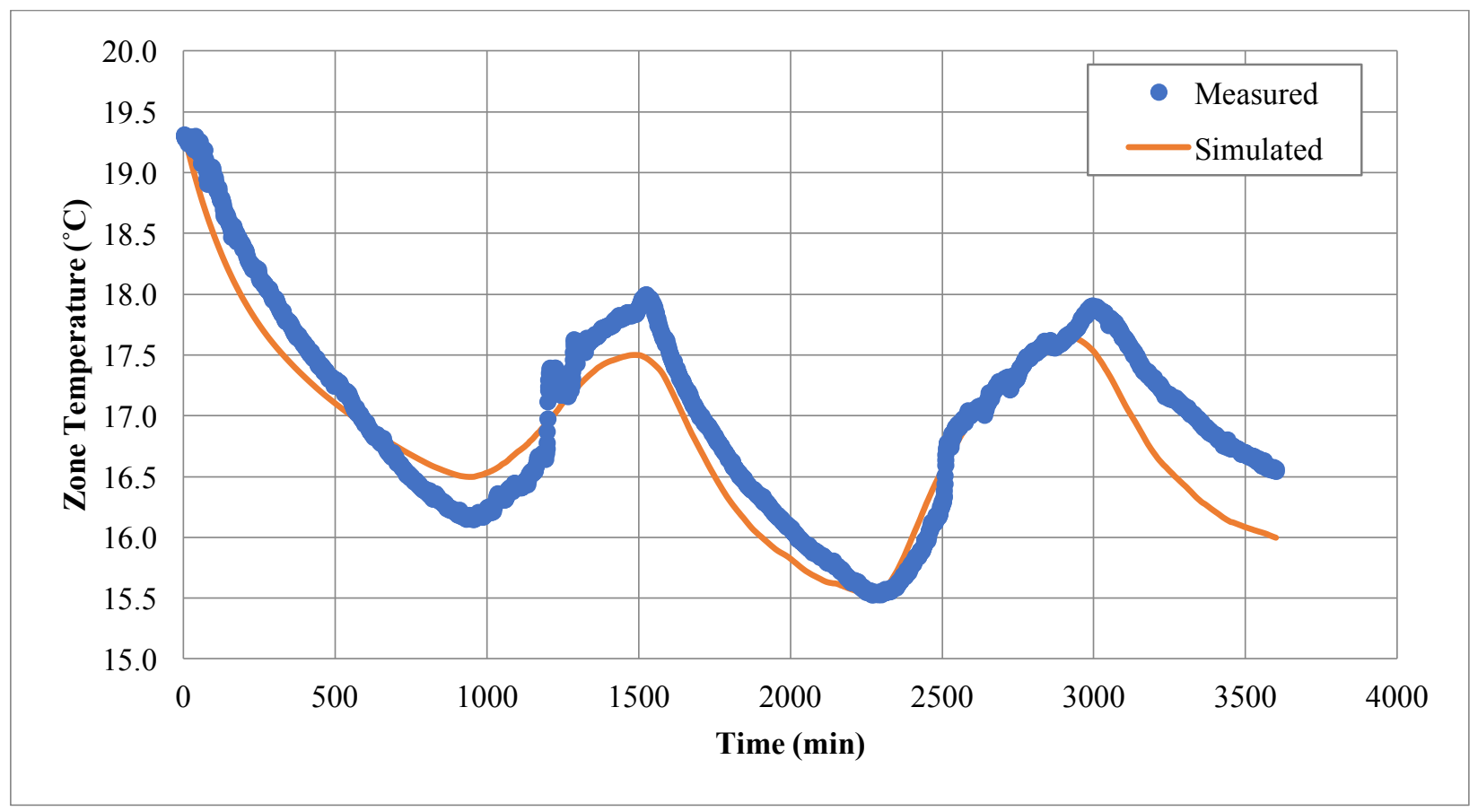

Figure 4-14: Short-term calibration performance $-R M S E=0.33^{\circ} \mathrm{C}(1.9 \%)$ 


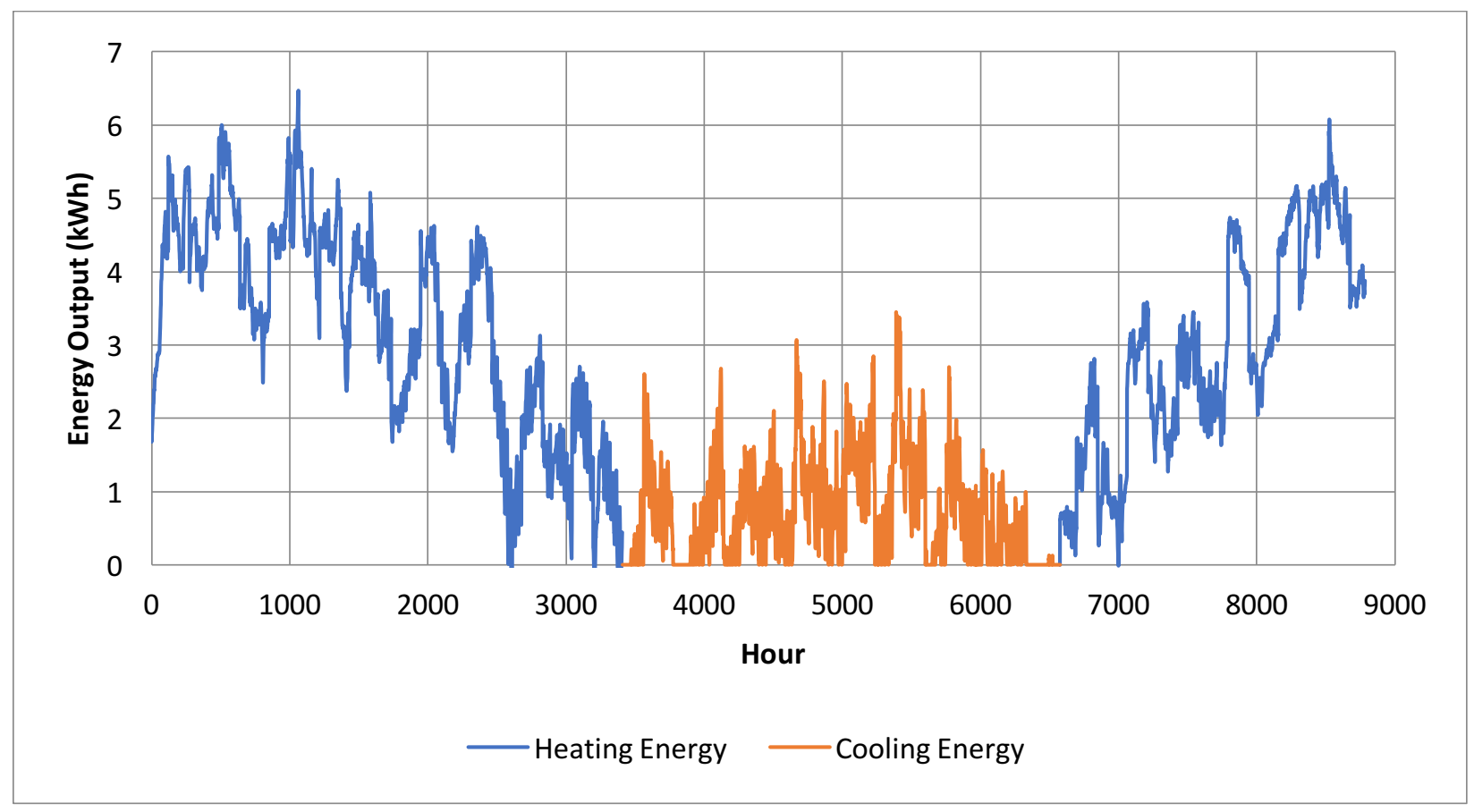

Figure 4-15: Annual simulated heating and cooling energy output

Table 4-10: Long-term calibration heating and cooling outputs and targets

\begin{tabular}{|l|c|c|c|}
\hline Season & Simulated Output (kWh) & Annual Target (kWh) & Percent Difference \\
\hline Heating & 16,174 & 16,251 & $-0.47 \%$ \\
\hline Cooling & 2,295 & 2,354 & $-2.51 \%$ \\
\hline
\end{tabular}

Table 4-11 summarizes the calibrated house parameters. Some parameters are very close to realworld values, while others vary more noticeably. Deviations from real-world values can be attributed to assumptions that do not fully capture real world conditions. Assumptions such as homogeneous wall construction, uniform and empty interior zone space, and infiltration constants all contribute to deviations from real-world values.

Nevertheless, all the calibrated parameters are within possible ranges for real-world building materials. Since these parameters produce a model that accurately simulates the operation of ASHA, they have been for all building simulations in the remainder of this work. 
Table 4-11: Calibrated house model parameters

\begin{tabular}{|c|c|c|}
\hline Parameter & $\begin{array}{l}\text { Calibrated } \\
\text { Value }\end{array}$ & Notes \\
\hline $\begin{array}{l}\text { Initial thermal } \\
\text { mass temperature }\end{array}$ & $19.9^{\circ} \mathrm{C}$ & $\begin{array}{l}\text { - Used for all wall sections, the ceiling, and the floor. } \\
\text { - Only had a significant impact on the short-term } \\
\text { calibration. }\end{array}$ \\
\hline $\begin{array}{l}\text { Wall thermal } \\
\text { conductivity }\end{array}$ & $0.011 \mathrm{~W} / \mathrm{m} \cdot \mathrm{K}$ & $\begin{array}{l}\text { - Used for all wall sections, the ceiling, and the floor. } \\
\text { - } \quad \text { Actual value estimated to be } 0.039 \mathrm{~W} / \mathrm{m} \bullet \mathrm{K}\end{array}$ \\
\hline Wall heat capacity & $1613 \mathrm{~J} / \mathrm{kg} \cdot \mathrm{K}$ & $\begin{array}{l}\text { - Used for all wall sections, the ceiling, and the floor. } \\
\text { - Within realistic range }(710-5,280 \mathrm{~J} / \mathrm{kg} \bullet \mathrm{K} \text { for } \\
\text { mineral fiber })\end{array}$ \\
\hline Wall thickness & $0.487 \mathrm{~m}$ & - Used for all wall sections, the ceiling, and the floor \\
\hline $\begin{array}{l}\text { Solar absorption of } \\
\text { the floor }\end{array}$ & 0.400 & $\begin{array}{l}\text { - Used when calculating the interior solar gains. } \\
\text { - Within realistic range for building materials }(0.4- \\
0.9)\end{array}$ \\
\hline $\begin{array}{l}\text { Solar emissivity of } \\
\text { the exterior walls }\end{array}$ & 0.050 & $\begin{array}{l}\text { - Used to calculate the sol-air temperature on all } \\
\text { exterior surfaces. } \\
\text { - Within realistic range for building materials ( } 0.05- \\
0.95)\end{array}$ \\
\hline $\begin{array}{l}\text { Wall overall heat } \\
\text { transfer coefficient }\end{array}$ & $\begin{array}{c}1.400 \\
\mathrm{~W} / \mathrm{m}^{2} \cdot \mathrm{K}\end{array}$ & $\begin{array}{l}\text { Less insulating than nominal value } \\
\left(0.177 \mathrm{~W} / \mathrm{m}^{2} \cdot \mathrm{K}\right) \text {, may account for thermal bridging }\end{array}$ \\
\hline $\begin{array}{l}\text { Ceiling overall heat } \\
\text { transfer coefficient }\end{array}$ & $\begin{array}{c}0.390 \\
\mathrm{~W} / \mathrm{m}^{2} \cdot \mathrm{K}\end{array}$ & $\begin{array}{l}\text { More insulating than nominal value } \\
\left(0.142 \mathrm{~W} / \mathrm{m}^{2} \cdot \mathrm{K}\right)\end{array}$ \\
\hline $\begin{array}{l}\text { Floor overall heat } \\
\text { transfer coefficient }\end{array}$ & $\begin{array}{c}0.142 \\
\mathrm{~W} / \mathrm{m}^{2} \cdot \mathrm{K}\end{array}$ & $\begin{array}{l}\text { - } \quad \text { More insulating than nominal value } \\
\left(0.284 \mathrm{~W} / \mathrm{m}^{2} \cdot \mathrm{K}\right)\end{array}$ \\
\hline $\begin{array}{l}\text { Window overall } \\
\text { heat transfer } \\
\text { coefficient }\end{array}$ & $\begin{array}{c}1.500 \\
\mathrm{~W} / \mathrm{m}^{2} \cdot \mathrm{K}\end{array}$ & $\begin{array}{l}\text { - } \quad \text { More insulating than nominal value } \\
\left(1.69 \mathrm{~W} / \mathrm{m}^{2} \bullet \mathrm{K}\right)\end{array}$ \\
\hline $\begin{array}{l}\text { Equivalent zone } \\
\text { heat capacity }\end{array}$ & $3.0 \mathrm{~kJ} / \mathrm{kg} \bullet \mathrm{K}$ & $\begin{array}{l}\text { - Slightly higher than the actual value for air (1.005 } \\
\mathrm{kJ} / \mathrm{kg} \cdot \mathrm{K} \text { at room temperature), however this is an } \\
\text { equivalent value that represents the air along with } \\
\text { all other building internals. }\end{array}$ \\
\hline
\end{tabular}




\subsection{Domestic Hot Water}

The simulated domestic hot water (DHW) system was based on the water heater currently installed in the ASH-B. This simulation was made possible in large part by previous work conducted in this research group (Amirirad, 2016). The water heater is a heat pump water heater (HPWH), with the heat pump unit attached directly to the top of the tank. The specifications of the water heater are presented in Table 4-12.

Table 4-12: A.O. Smith HPWH Specifications (A.O.Smith Enterprises Ltd., 2015)

\begin{tabular}{|l|c|}
\hline Manufacturer and model & A.O.Smith - SHPT-50 \\
\hline Capacity & $189 \mathrm{~L}(50 \mathrm{gal})$ \\
\hline Rated energy factor - Heat pump mode & 2.78 \\
\hline Rated energy factor - Hybrid mode & 2.75 \\
\hline Rated energy factor - Electric mode & 0.89 \\
\hline Tank height & $1.60 \mathrm{~m}$ \\
\hline Tank diameter & $0.56 \mathrm{~m}$ \\
\hline Exterior surface area & $2.65 \mathrm{~m}^{2}$ \\
\hline Rated compressor power consumption & $624 \mathrm{~W}(2.8 \mathrm{~A}$ at $240 \mathrm{~V})$ \\
\hline Electric mode power consumption & $1500 \mathrm{~W}($ Amirirad, 2016) \\
\hline Overall U value (calculated) & $0.877 \mathrm{~W} / \mathrm{m}^{2} \cdot \mathrm{K}($ Amirirad, 2016) \\
\hline
\end{tabular}

For the simulations conducted in this work, the water heater was operated in electric mode. This was done such that the effect of a HPWH on the heating and cooling consumption of the building not distract from other changes in energy consumption that are a result of the investigated EMS control schemes.

Figure 4-16 displays the compressor power consumption when the water heater is operating in heat pump mode over a one-week period. Based on the average of six months of data collection, the power consumption of the HPWH is approximately $564 \mathrm{~W}$ when operating in heat pump mode, and $1500 \mathrm{~W}$ in electric mode. 


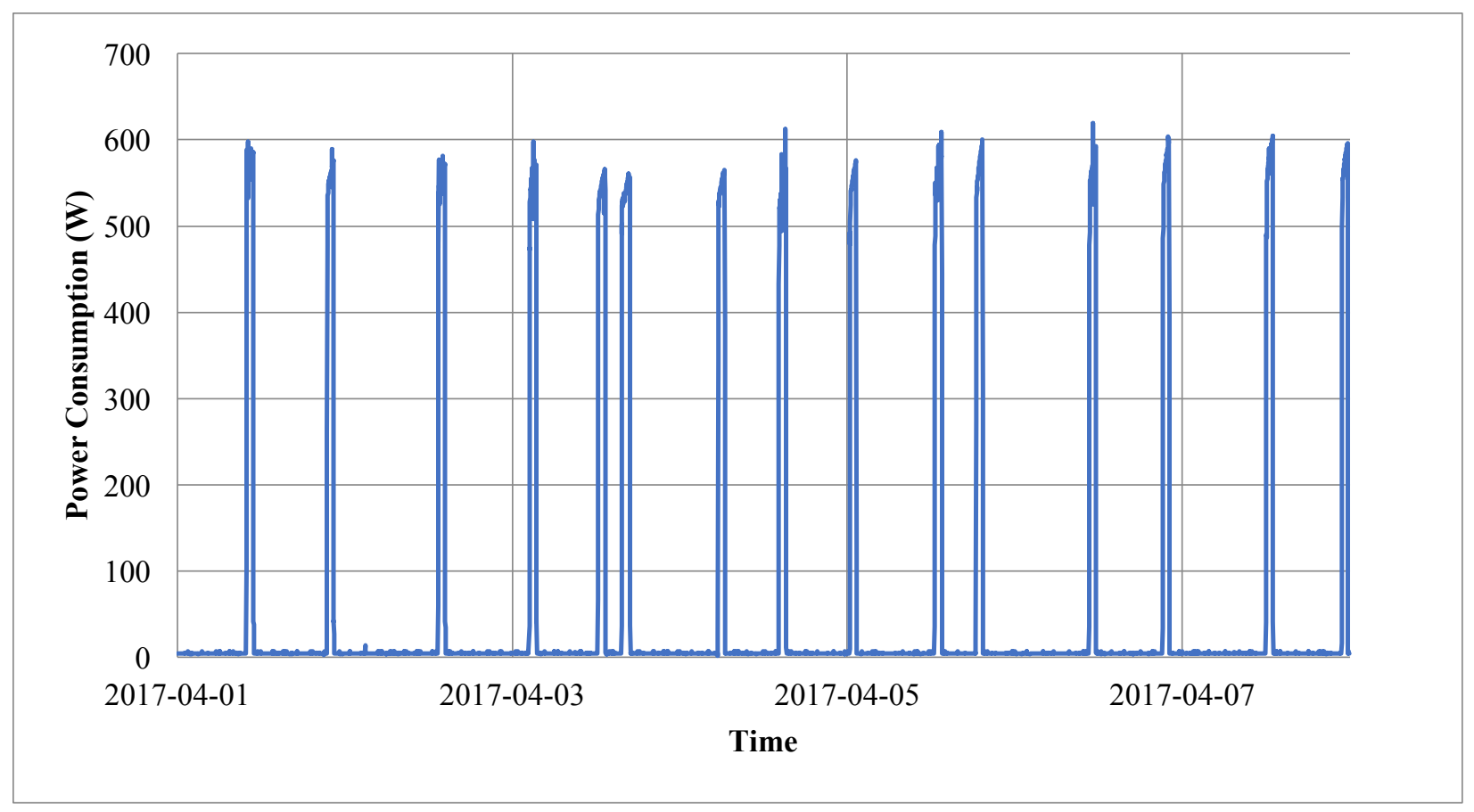

Figure 4-16: A.O.Smith power consumption in heat pump mode over a one-week period

\subsubsection{Domestic Hot Water Model Inputs}

A water draw profile was obtained from IEA Annex 42 data for use in simulations of Canadian and European homes (Knight, Kreutzer, Manning, Swinton, \& Ribberink, 2007). The profile was based on an average draw of $200 \mathrm{~L}$ of hot water per day. A one-minute draw profile schedule was used.

Water inlet data was obtained experimentally from the ASH. Measurements of water inlet temperature to the house were averaged over each month, and are displayed in Figure 4-17. 


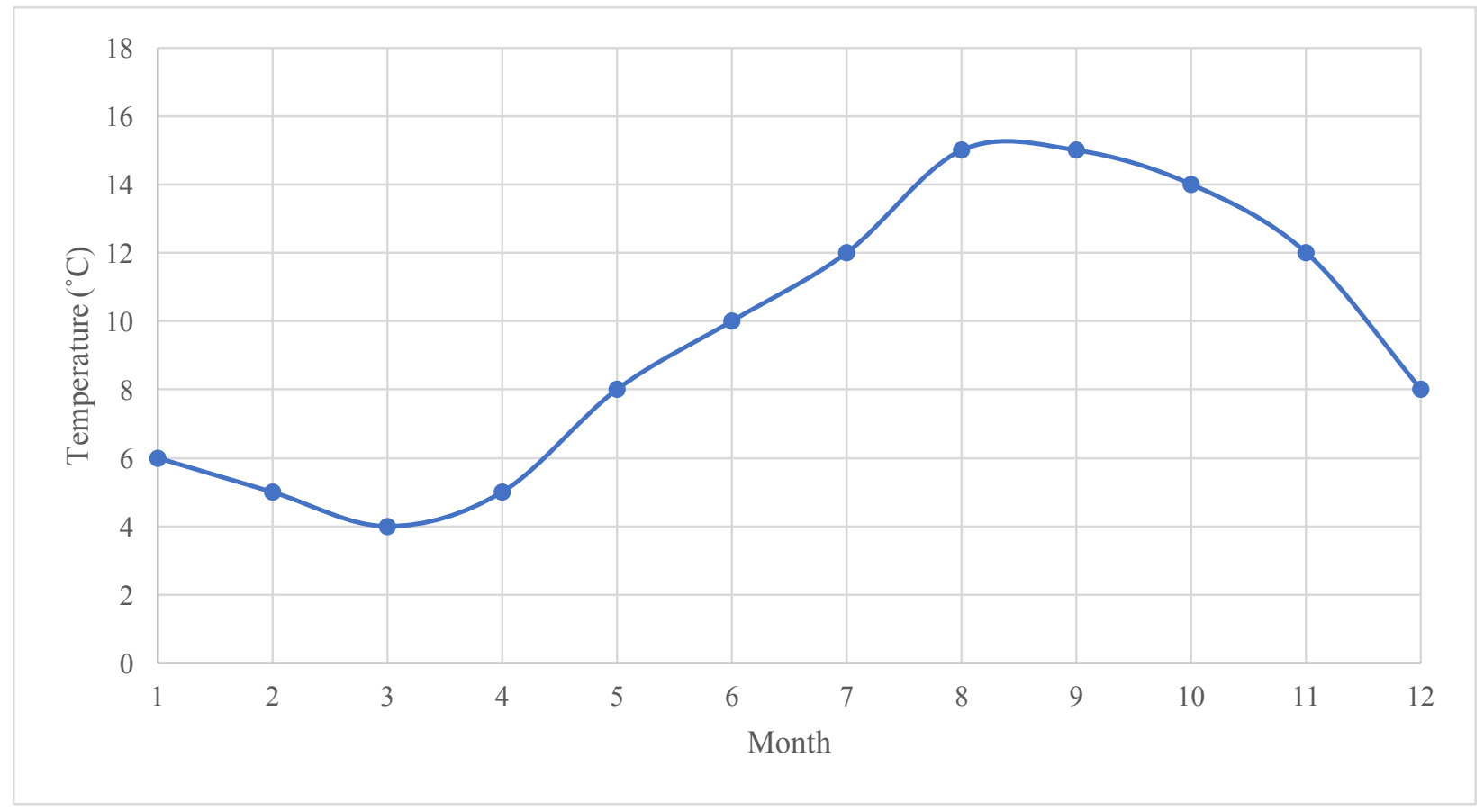

Figure 4-17: Monthly average water inlet temperature (Amirirad, 2016)

\subsubsection{Domestic Hot Water Model Procedure}

A lumped capacitance model was used to model the water heater. In this case, the significant thermal mass is the water contained in the tank. Equation 4-45 defines the basis for this model.

$$
C_{m, w} \frac{d T_{w}}{d t}=\dot{q}_{i n, w}-\dot{q}_{\text {out }, w}-\dot{q}_{\text {loss }, \text { tank }}
$$

Where $C_{m, w}$ is the thermal capacitance of water, $\dot{q}_{i n, w}$ is the heat energy provided to the tank by the water heater, $\dot{q}_{\text {out }, w}$ is the energy extracted from the tank by withdrawing water, and $\dot{q}_{\text {loss,tank }}$ is the heat loss of the tank to the surroundings. Equations 4-46 to 4-48 show the breakdown of each of these three heat components.

$$
\dot{q}_{i n, w}=\dot{W}_{\text {in,elec }} \cdot \eta_{\text {tank }, \text { tot }}
$$

Where $\dot{W}_{\text {in,elec }}$ is the electrical energy consumed by the water heater, and $\eta_{\text {tank,tot }}$ is the overall efficiency of the water heater. For this work, the rated energy factor of 0.89 was used as the overall efficiency of the water heater. 


$$
\dot{q}_{o u t, w}=\dot{m} c_{p, w}\left(T_{o u t, w}-T_{i n, w}\right)
$$

Where $\dot{m}$ is the mass flowrate of water, defined by the water draw schedule, $c_{p, w}$ is the specific heat capacity of water $(4.18 \mathrm{~kW} / \mathrm{kg} \cdot \mathrm{K})$, and $T_{o u t, w}$ and $T_{i n, w}$ are the outlet and inlet water temperatures, respectively. The outlet water temperature was taken to be equal to the average tank temperature at each time-step.

$$
\dot{q}_{\text {loss, } \text { tank }}=U A\left(T_{w}-T_{a m b}\right)
$$

Where $U$ is the overall heat transfer coefficient of the tank, which has been experimentally measured to be $0.877 \mathrm{~W} / \mathrm{m}^{2} \cdot \mathrm{K}$ (Amirirad, 2016). $A$ is the outer surface area of the tank, and $T_{w}$ and $T_{a m b}$ are the water and ambient temperature, respectively.

\subsection{Renewable Energy Systems}

Two types of residential renewable energy systems were considered for this work: solar PV and small-scale wind turbines. The solar PV system was modeled using an ANN black box model, trained with experimental data collected at the ASH. Due to insufficient available wind data, the small-scale wind turbine was modeled simply using its manufacturer's power curve.

\subsubsection{Solar Photovoltaic Model}

The power generated by a roof-mounted solar PV system was modeled using data collected at the ASH. The ANN was generated and trained in MATLAB. Data from the second half of 2016 was used to train the ANN, covering summer and winter outdoor conditions. Direct, diffuse, and global horizontal irradiance were all used as inputs to the ANN, along with ambient temperature. The output of the ANN was power generated by the solar PV system.

Using only a single hidden layer, an analysis on the number of neurons used by the ANN was

performed. The analysis used only the coefficient of determination $\left(\mathrm{R}^{2}\right)$ as a performance metric, as the RMSE did not accurately represent the performance of this model. Figure 4-18 displays the 
performance of the solar PV ANN as a function of hidden layer size, where the error bars indicate the standard deviation of 30 training trials.

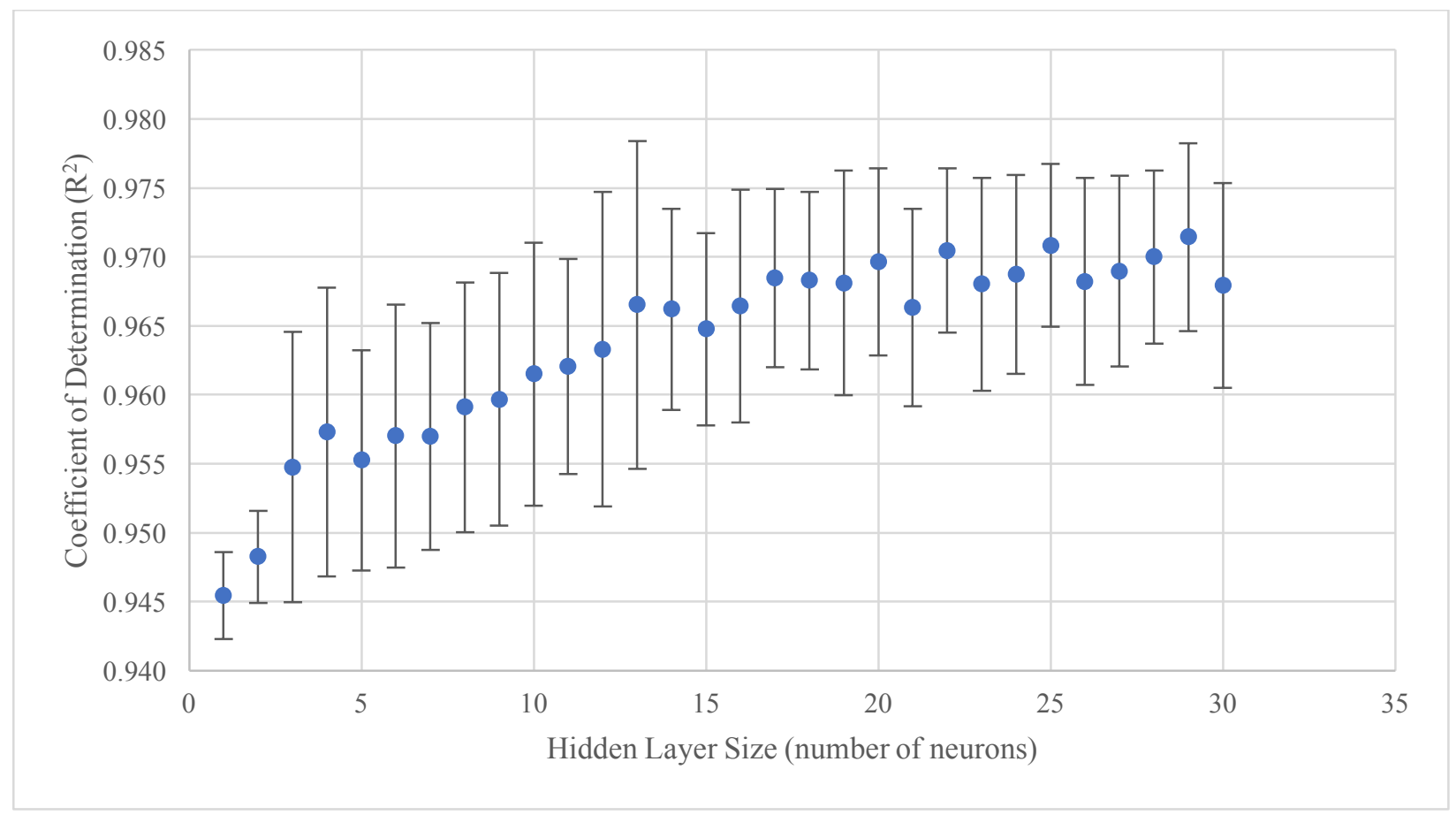

Figure 4-18: Solar PV ANN Performance

A neural network with 25 neurons in a single hidden layer was selected as the most accurate candidate. The performance of the final solar PV ANN, with $\mathrm{R}^{2}=0.9764$ from training, is shown against measured solar PV output in Figure 4-19.

It has been assumed that PV generation scales proportional to system size (number of panels, or total rated output). Therefore, this ANN has been used to represent any house-sized solar PV system $(\sim 1-10 \mathrm{~kW})$ by multiplying its output by the ratio of the target system size to the trained system size. While House B has $4.08 \mathrm{~kW}$ of roof-mounted PV, simulations conducted throughout this report utilize a variety of system sizes to represent different cases, and perform sensitivity analyses. 


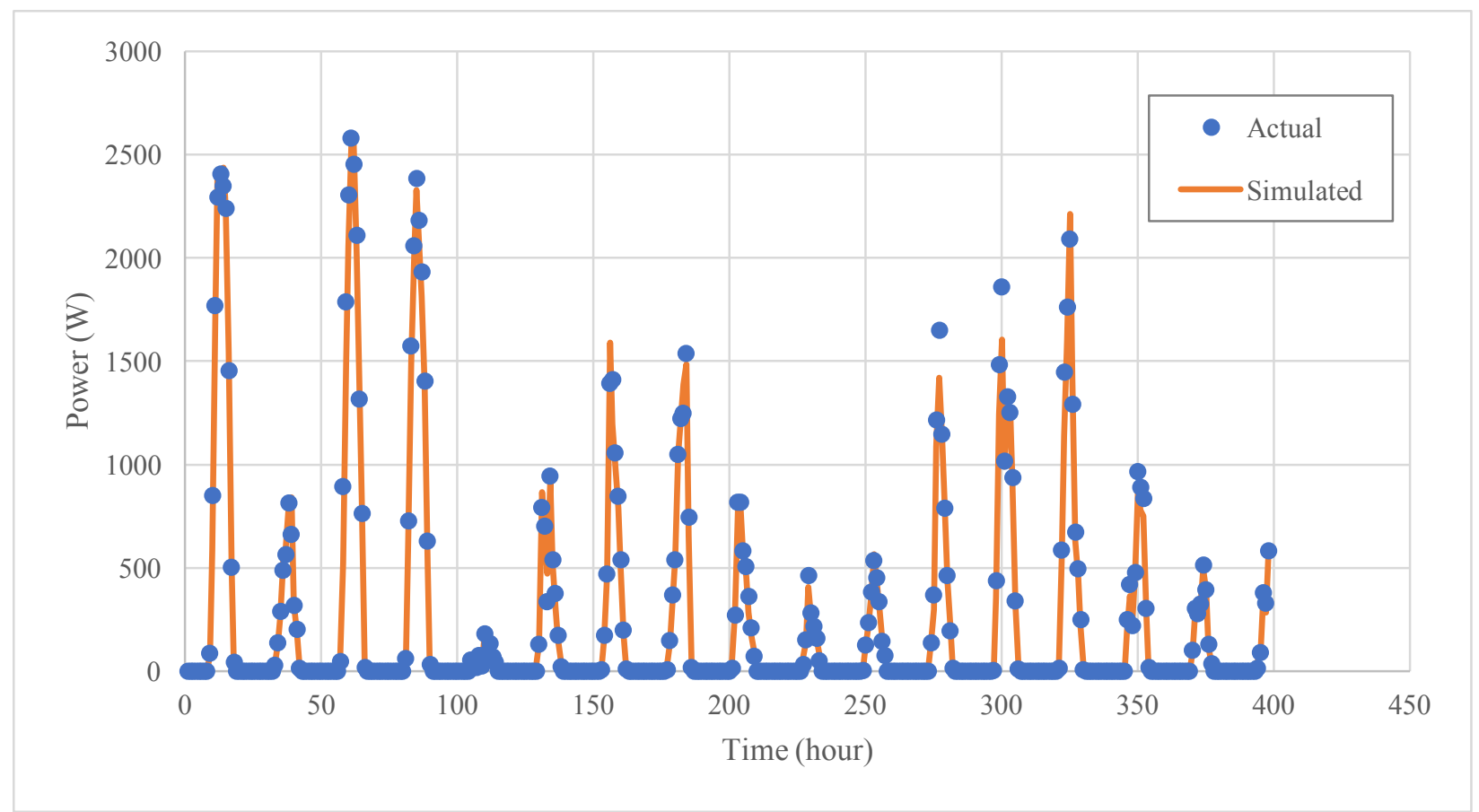

Figure 4-19: Solar PV ANN Performance on sample hourly dataset $\left(R^{2}=0.9906\right)$

\subsubsection{Small-Wind Turbine Model}

The first step of the wind turbine power output estimation model was to calculate the wind speed at the hub height of the turbine. The Lufft WS600 weather station, which measured the wind data used for this work, is mounted at a height of $3.66 \mathrm{~m}$ (12ft), while the small-scale wind turbine adjacent to the ASH is mounted at approximately $18.3 \mathrm{~m}$ (60ft). Equation 4-49 was used to correct for as the wind speed for the turbine hub height (Gipe, 1999).

$$
u=u_{o}\left(\frac{h}{h_{o}}\right)^{a}
$$

Where $u$ is the wind speed at the hub height of the turbine $(h) . u_{o}$ is the wind speed measurement taken at $h_{o}$, the height of the measurement device. $a$ is the surface roughness exponent, which was assumed to be 0.25 for all calculations, a value applicable to a suburban or woodland setting (Gipe, 1999). 
The power output of the wind turbine was then estimated based on experimental verification conducted by the Small Wind Certification Council (SWCC). Figure 4-20 displays three smallwind turbine power curves considered for this work.

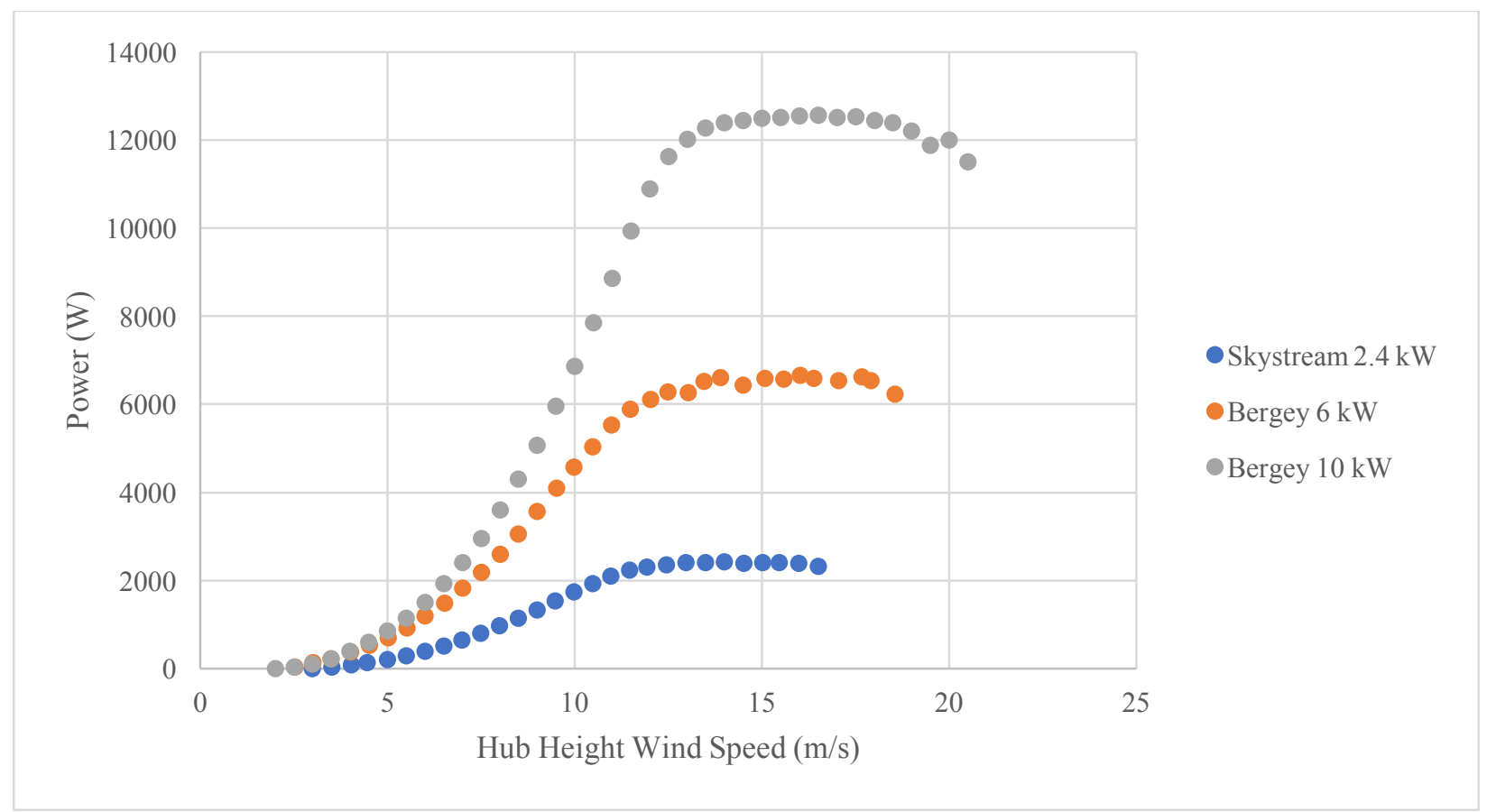

Figure 4-20: Experimentally determined small wind turbine power curves (SWCC, 2016)

\subsection{Battery Model}

A simplified battery model was used for all EMS simulations. The model took into consideration battery charging and discharging efficiencies, but neglected certain operating parameters such as battery temperature. The battery's energy at a given time step would be evaluated based on a charging or discharging command, and the amount of associated energy. Equations 4-50 and 4-51 display how the internal energy of the battery is calculated at each time step when charging or discharging, respectively.

$$
\begin{gathered}
E_{\text {batt }, \text { int }}^{t+1}=E_{\text {batt,int }}^{t}+\eta_{c} P_{\text {grid }} \Delta t \\
E_{\text {batt,int }}^{t+1}=E_{\text {batt,int }}^{t}-\frac{P_{\text {grid }}}{\eta_{d c}} \Delta t
\end{gathered}
$$


Where $E_{\text {batt,int }}^{t}$ is the internal energy of the battery at time step $t, P_{\text {grid }}$ is the power on the gridside of the battery's inverter/charger, and $\eta_{c}$ and $\eta_{d c}$ are the charging and discharging efficiencies respectively. For all calculations, the charging and discharging efficiencies were assumed to be $85 \%$ and $95 \%$, respectively, based on experimental data. These values were obtained from experimental measurements taken at the $\mathrm{ASH}$, and they represent the lower-end of battery performance, relative to rapid advances in the industry.

A side note on battery health, and ensuring longevity. Lead-acid batteries are notorious for their very particular charging procedure; their lifespan is directly proportional to how they are used, particularly in terms of rate of charge, cycling, and depth of discharge.

Charging a lead-acid battery is analogous to filling a bucket to the brim with water using a fire hose - while a high flow rate will effectively fill the bucket at first, a point is reached when the flow is too great, and water begins to spill out over the brim. In the case of a lead-acid battery, this phenomenon is called gassing, which is damaging to the battery and shortens its lifespan. At higher rates of charge, battery gassing begins at lower and lower states of charge. Because of this, leadacid battery banks are charged in three stages: bulk, absorption, and float, each stage with a slower rate of charge than the preceding (De Bruyn, 2017). Lithium ion batteries on the other hand are far more flexible with their charging and discharging procedure, a fact made clear by rapid rates of charge possible from a Tesla Supercharger.

For the most part, the EMS analyses ignore battery charging requirements, and assume that the battery banks can handle any rate of charge or discharge, whenever required. Some of the control methods investigated may cause the rate of charge to fluctuate to a degree that would be damaging to a lead-acid battery. It is therefore assumed that cases with rapid changes in charging and discharging states would require lithium ion batteries in the real world. However, in all cases the same charging and discharging efficiencies ( $85 \%$ and $95 \%)$ have been used. These efficiencies are lower than typically expected of lithium ion batteries, and therefore produce a conservative, worstcase scenario estimate of potential savings. 


\subsection{Non-HVAC Load Simulation}

Non-HVAC loads were simulated using stochastic modelling approaches. This method of load profile simulation is in accordance with that which is set out by the IEA Annex 42 (Armstrong, Swinton, Ribberink, Beausoleil-Morrison, \& Millette, 2009). Each appliance was simulation at 5minute intervals for one year. At each time step, a randomly generated number would be compared to the TOU probability curve of the given appliance (Section 4.10.1). If the random number was within the probability of occurrence, then the appliance turned on, and remained on until its cycle time was reached. Otherwise, the appliance remained off. The non-HVAC load simulations considered occupant behaviour (captured in the TOU probability curves), instantaneous power draw, appliance cycle duration, annual energy consumption, and number of cycles per year.

Simulations for three different cases were carried out, representing low, medium, and high energy consumers. Household characteristics for each of the three cases, including their number of appliances, are summarized in Table 4-13. The use factors were chosen such that the low, medium, and high energy houses agreed with the actual annual energy consumption of Canadian homes (NRCan, 2013).

Table 4-13: Summary of the three house configurations used for the simulation of non-HVAC loads (Armstrong, Swinton, Ribberink, Beausoleil-Morrison, \& Millette, 2009)

\begin{tabular}{|c|c|c|c|c|c|c|c|c|c|}
\hline \multirow[b]{2}{*}{ Load } & \multicolumn{3}{|c|}{ Low Energy House } & \multicolumn{3}{|c|}{ Medium Energy House } & \multicolumn{3}{|c|}{ High Energy House } \\
\hline & $\begin{array}{l}\text { No. } \\
\text { Appl. }\end{array}$ & Factor & $\begin{array}{c}\text { kWh } \\
/ \mathbf{y r}\end{array}$ & $\begin{array}{l}\text { No. } \\
\text { Appl. }\end{array}$ & Factor & $\begin{array}{c}\text { kWh } \\
/ \mathbf{y r}\end{array}$ & $\begin{array}{l}\text { No. } \\
\text { Appl. }\end{array}$ & Factor & $\begin{array}{c}\mathbf{k W h} \\
/ \mathbf{y r}\end{array}$ \\
\hline Refrigerator & 1 & 1.0 & 801.0 & 1 & 1.0 & 801.0 & 2 & 1.0 & 1602.0 \\
\hline Freezer & 0 & 0.0 & 0.0 & 1 & 1.0 & 614.0 & 1 & 1.3 & 798.2 \\
\hline Dishwasher & 1 & 0.8 & 57.6 & 1 & 1.3 & 93.6 & 1 & 1.7 & 122.4 \\
\hline Washer & 1 & 0.8 & 60.8 & 1 & 1.3 & 98.8 & 1 & 2.0 & 152.0 \\
\hline Dryer & 1 & 0.6 & 592.8 & 1 & 1.3 & 1284.4 & 1 & 2.0 & 1976.0 \\
\hline Range & 1 & 1.0 & 769.0 & 1 & 1.0 & 769.0 & 1 & 1.4 & 1076.6 \\
\hline Misc. Appl. & - & 0.8 & 1516.8 & - & 1.3 & 2464.8 & & 1.7 & 3223.2 \\
\hline Lighting & $141 \mathrm{~m}^{2}$ & 0.5 & 1015.2 & $141 \mathrm{~m}^{2}$ & 1.0 & 2030.4 & $282 \mathrm{~m}^{2}$ & 1.0 & 4060.8 \\
\hline $\begin{array}{l}\text { Total } \\
(\mathrm{kWh} / \mathrm{yr})\end{array}$ & & & 4813.2 & & & 8156.0 & & & 13011.2 \\
\hline $\begin{array}{l}\text { Daily Avg. } \\
(\mathrm{kWh} / \mathrm{d})\end{array}$ & & & 13.2 & & & 22.3 & & & 35.6 \\
\hline
\end{tabular}




\subsubsection{Appliance Probability Curves}

Due to the availability of data, hourly appliance usage characteristics were obtained for American homes (U.S. Department of Energy, 2011), and not Canadian homes. However, for the purposes of this work, any direct effect of climate on non-HVAC loads has been ignored. Therefore, the major error associated with using American instead of Canadian data would be associated in occupant behavior characteristics, which are assumed to be similar enough to suffice for this work.

Probability curves for each appliance were derived from their normalized time-of-use curves. These curves represent the shape of the probability curve, but the probability magnitude had to be derived based on annual energy end-use targets. These targets, along with the number of annual cycles, are summarized in Table 4-14. Note that the same refrigerator is assumed to be used in all houses, but the high-energy house has two units.

Table 4-14: Annual appliance target characteristics (Armstrong, Swinton, Ribberink, BeausoleilMorrison, \& Millette, 2009)

\begin{tabular}{|c|c|c|c|c|}
\hline Appliance & Power (W) & $\begin{array}{l}\text { Cycle duration } \\
\text { (min) }\end{array}$ & $\begin{array}{l}\text { Target energy } \\
\text { (kWh/year) }\end{array}$ & $\begin{array}{l}\text { Target cycles } \\
\text { per year }\end{array}$ \\
\hline Refrigerator & 265 (peak) & $\begin{array}{l}70 \text { (normal) } \\
105 \text { (defrost) }\end{array}$ & $\begin{array}{c}801 \text { (low) } \\
801 \text { (medium) } \\
1602 \text { (high) }\end{array}$ & ---- \\
\hline Freezer & $\begin{array}{c}---- \\
202 \text { (peak) } \\
263 \text { (peak) }\end{array}$ & $\begin{array}{l}70 \text { (normal) } \\
105 \text { (defrost) }\end{array}$ & $\begin{array}{c}0 \text { (low) } \\
614 \text { (medium) } \\
798 \text { (high) }\end{array}$ & ---- \\
\hline Dishwasher & 467 & 30 to 45 & $\begin{array}{c}58 \text { (low) } \\
94 \text { (medium) } \\
122 \text { (high) }\end{array}$ & $\begin{array}{l}200 \\
322 \\
418\end{array}$ \\
\hline Clothes Washer & 505 & 30 & $\begin{array}{c}61 \text { (low) } \\
99 \text { (medium) } \\
152 \text { (high) }\end{array}$ & $\begin{array}{l}242 \\
392 \\
601\end{array}$ \\
\hline Clothes Dryer & 4115 & 30 to 60 & $\begin{array}{c}593 \text { (low) } \\
1284 \text { (medium) } \\
1976 \text { (high) }\end{array}$ & $\begin{array}{l}192 \\
416 \\
640\end{array}$ \\
\hline Range & 1600 & 15 to 70 & $\begin{array}{c}769 \text { (low) } \\
769 \text { (medium) } \\
1077 \text { (high) }\end{array}$ & $\begin{array}{l}678 \\
678 \\
950\end{array}$ \\
\hline
\end{tabular}


Using the different appliance normalized TOU operation curves, the probability curves were derived using Equation 4-52 (Armstrong, Swinton, Ribberink, Beausoleil-Morrison, \& Millette, 2009).

$$
\mathrm{P}_{a p p l}=\frac{f_{T O U}}{C F}
$$

Where $\mathrm{P}_{a p p l}$ is the probability curve for a given appliance, $f_{T O U}$ is the faction of total daily usage (from TOU curves), and $C F$ is the chance factor. Chance factors were calculated via a calibration process, in which annual energy consumption and number of cycles were compared to target values. To calibrate the chance factors, each appliance simulation was run 30 times at varying chance factor values, and the average annual energy consumption and number of cycles were compared to the target values shown in Table 4-14.

The following sections summarize the calculated chance factors for each appliance under the low, medium, and high energy use houses, along with their calculated probability curves.

\subsubsection{Refrigerator and Freezer}

The refrigerator and freezer were simulated slightly differently than other appliances. It was assumed that the fridge would cycle on and off based on a fixed cycle length (70 min). The cycle was interpreted to be composed of 35 minutes of power consumption, followed by 35 minutes of idle time, repeated continuously throughout the year. The probability curve was then used in order to stochastically determine when the defrost cycle would run. Figure 4-21 displays the impact of the chance factor on the annual energy consumption of the refrigerator, and Table 4-15 shows the calibrated simulated annual energy consumption. 


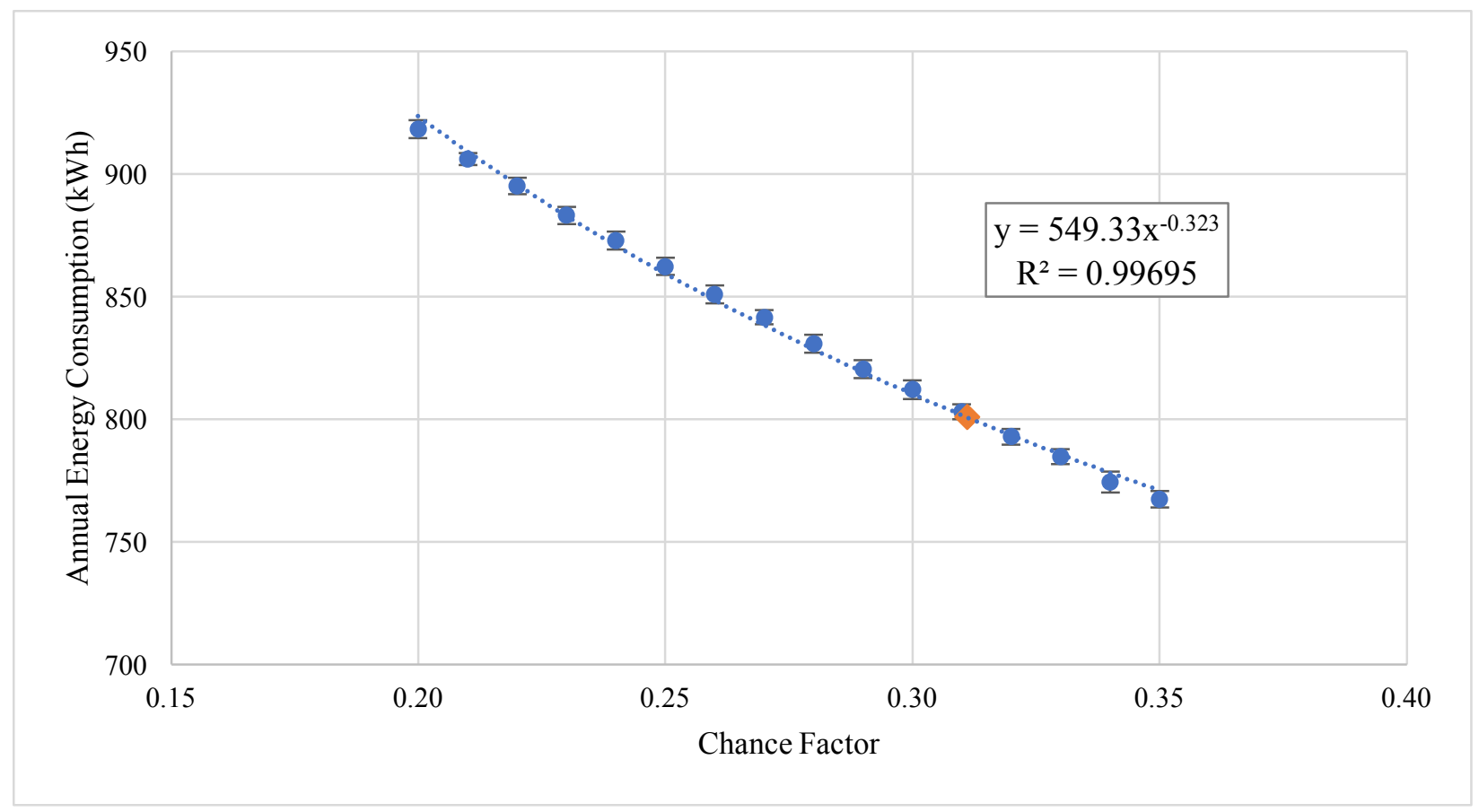

Figure 4-21: Refrigerator chance factor determination. Calibrated value $-C F=0.31$

Table 4-15: Refrigerator simulation performance $(C F=0.31)$

\begin{tabular}{|c|c|c|}
\hline House type & $\begin{array}{c}\text { Target annual energy } \\
\text { consumption }(\mathbf{k W h} / \mathbf{y r})\end{array}$ & $\begin{array}{c}\text { Simulated average annual } \\
\text { energy consumption }(\mathbf{k W h} / \mathbf{y r})\end{array}$ \\
\hline All & 801.0 & $801.9(\sigma=3.85, \mathrm{n}=30)$ \\
\hline
\end{tabular}

Figure 4-22 shows a sample daily averaged simulated refrigerator load profile. Note that because the refrigerator was assumed to operate on a fixed-cycle basis, it's operation is much less influenced by the occupant-driven probability curve than other appliances. 


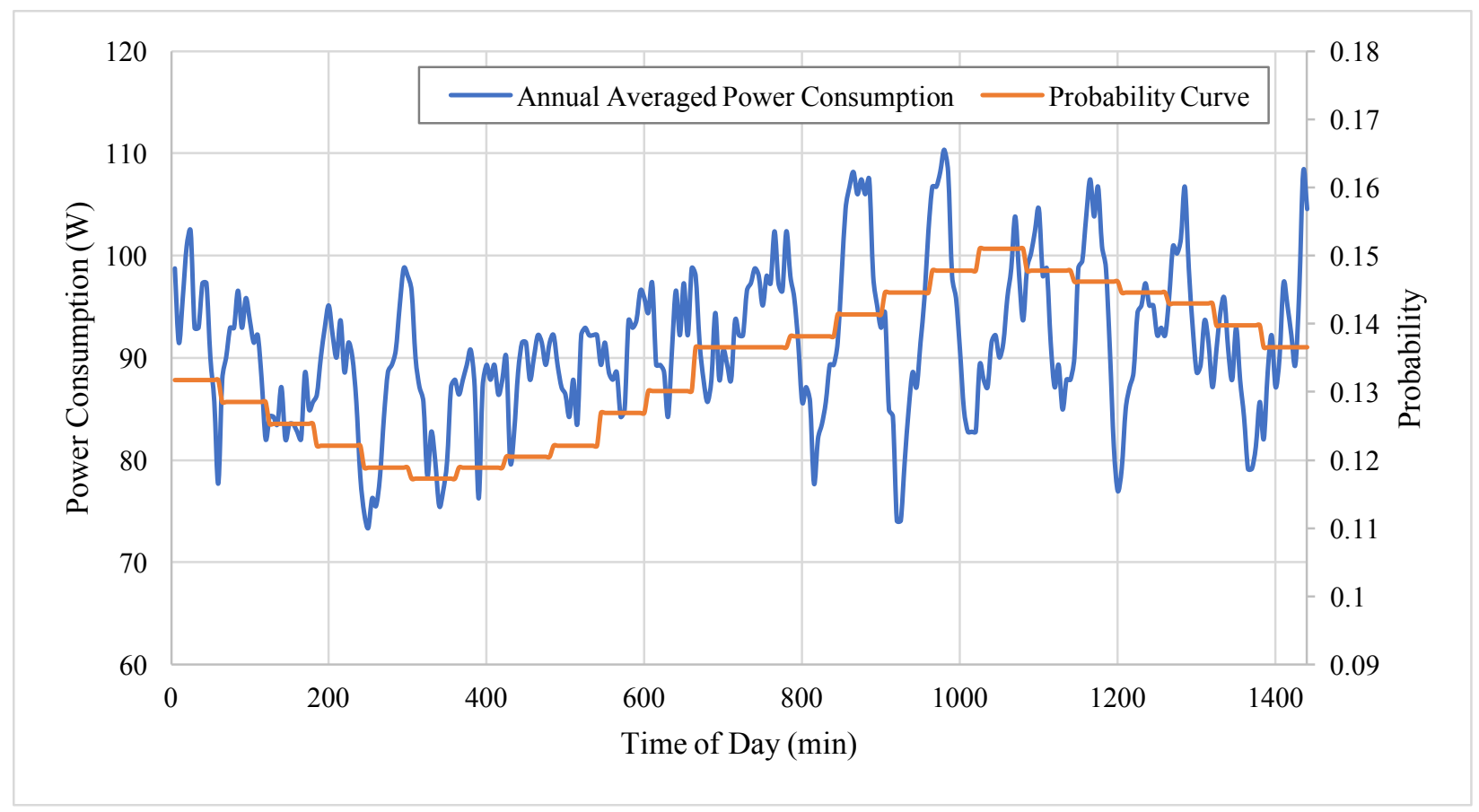

Figure 4-22: Sample refrigerator daily averaged load profile and probability curve

The freezer's power consumption was simulated using the same approach as the refrigerator simulation. However, two different freezers - with medium (202W) and high (265W) power consumption - were considered for the medium and high energy consumers respectively. The calculated chance factor, and energy consumption targets and outputs, are summarized in Table 4-16. The sample profile of the freezer is very similar to that of the refrigerator, and is therefore not displayed.

Table 4-16: Freezer simulation performance $(C F=0.31)$

\begin{tabular}{|c|c|c|}
\hline House type & $\begin{array}{c}\text { Target annual energy } \\
\text { consumption }(\mathbf{k W h} / \mathbf{y r})\end{array}$ & $\begin{array}{c}\text { Simulated average annual } \\
\text { energy consumption }(\mathbf{k W h} / \mathbf{y r})\end{array}$ \\
\hline Medium & 614.0 & $611.4(\sigma=3.07, \mathrm{n}=30)$ \\
\hline High & 798.0 & $796.6(\sigma=3.96, \mathrm{n}=30)$ \\
\hline
\end{tabular}

\subsubsection{Dishwasher}

Unlike the refrigerator and freezer, the dishwasher did not operate on a fixed-cycle basis. Instead, simulations depended entirely on the occupant-driven probability curve to determine when the dishwasher was on or off. When the dishwasher was on, it consumed a constant amount of power. 
Its cycle duration was randomly selected using a uniform distribution between $30-45$ minutes. Three different chance factors were calculated for each of the three house types. Table 4-17 summarizes the dishwasher simulation output and calibrated chance factors, while Figure 4-23 shows a sample daily averaged load profile.

Table 4-17: Dishwasher simulation performance

\begin{tabular}{|c|c|c|c|c|c|}
\hline & \multicolumn{2}{|c|}{$\begin{array}{c}\text { Annual energy consumption } \\
(\mathbf{k W h} / \mathbf{y r})\end{array}$} & Total number of cycles per year & \\
\hline $\begin{array}{c}\text { House } \\
\text { type }\end{array}$ & Target & Simulated & Target & Simulated & $\begin{array}{c}\text { Chance } \\
\text { Factor }\end{array}$ \\
\hline Low & 58 & $\begin{array}{c}56.5 \\
(\sigma=3.86, \mathrm{n}=30)\end{array}$ & 200 & $\begin{array}{c}194 \\
(\sigma=12.7, \mathrm{n}=30)\end{array}$ & 22.1 \\
\hline Medium & 94 & $\begin{array}{c}96.2 \\
(\sigma=4.92, \mathrm{n}=30)\end{array}$ & 322 & $\begin{array}{c}329 \\
(\sigma=15.9, \mathrm{n}=30)\end{array}$ & 13.1 \\
\hline High & 122 & $\begin{array}{c}124.4 \\
(\sigma=5.89, \mathrm{n}=30)\end{array}$ & 418 & $\begin{array}{c}425 \\
(\sigma=19.9, \mathrm{n}=30)\end{array}$ & 9.9 \\
\hline
\end{tabular}

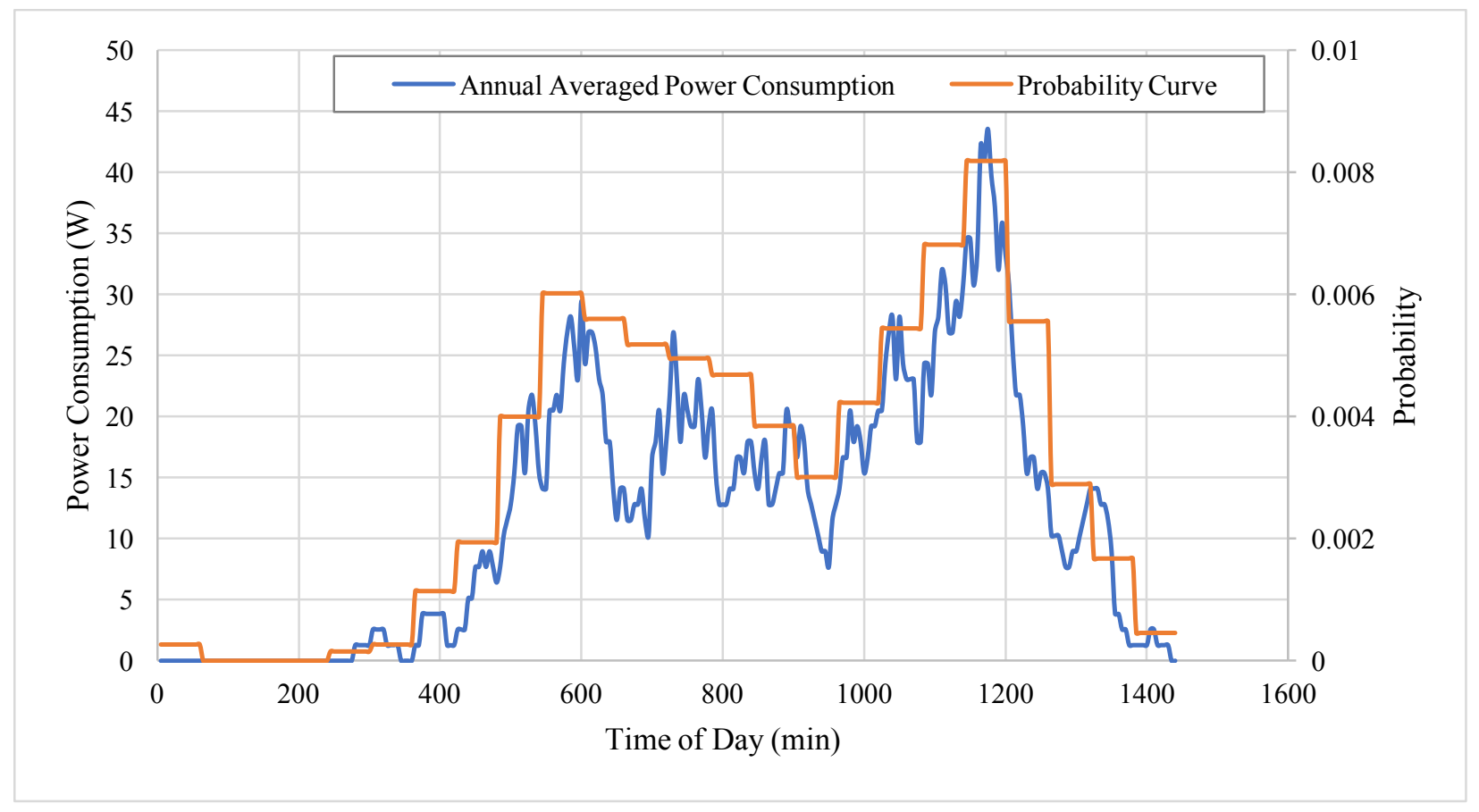

Figure 4-23: Sample dishwasher daily averaged load profile and probability curve (medium energy house) 


\subsubsection{Clothes Washer}

Like the dishwasher, the clothes washer's operation was simulated based entirely on its probability curve. It was assumed to have a cycle time of 30 minutes. The three chance factors, along with annual energy consumption and number of cycles for each condition, are summarized in Table 4-18, while Figure 4-24 displays a sample daily averaged load profile.

Table 4-18: Clothes washer simulation performance

\begin{tabular}{|c|c|c|c|c|c|}
\hline & \multicolumn{2}{|c|}{$\begin{array}{c}\text { Annual energy consumption } \\
(\mathbf{k W h} / \mathbf{y r})\end{array}$} & Total number of cycles per year & \\
\hline $\begin{array}{c}\text { House } \\
\text { type }\end{array}$ & Target & Simulated & Target & Simulated & $\begin{array}{c}\text { Chance } \\
\text { Factor }\end{array}$ \\
\hline Low & 61 & $\begin{array}{c}60.5 \\
(\sigma=3.77, \mathrm{n}=30)\end{array}$ & 242 & $\begin{array}{c}239 \\
(\sigma=14.9, \mathrm{n}=30)\end{array}$ & 18.1 \\
\hline Medium & 99 & $\begin{array}{c}99.1 \\
(\sigma=4.52, \mathrm{n}=30)\end{array}$ & 392 & $\begin{array}{c}392 \\
(\sigma=17.9, \mathrm{n}=30)\end{array}$ & 10.7 \\
\hline High & 152 & $\begin{array}{c}155.3 \\
(\sigma=4.91, \mathrm{n}=30)\end{array}$ & 601 & $\begin{array}{c}615 \\
(\sigma=19.4, \mathrm{n}=30)\end{array}$ & 6.8 \\
\hline
\end{tabular}

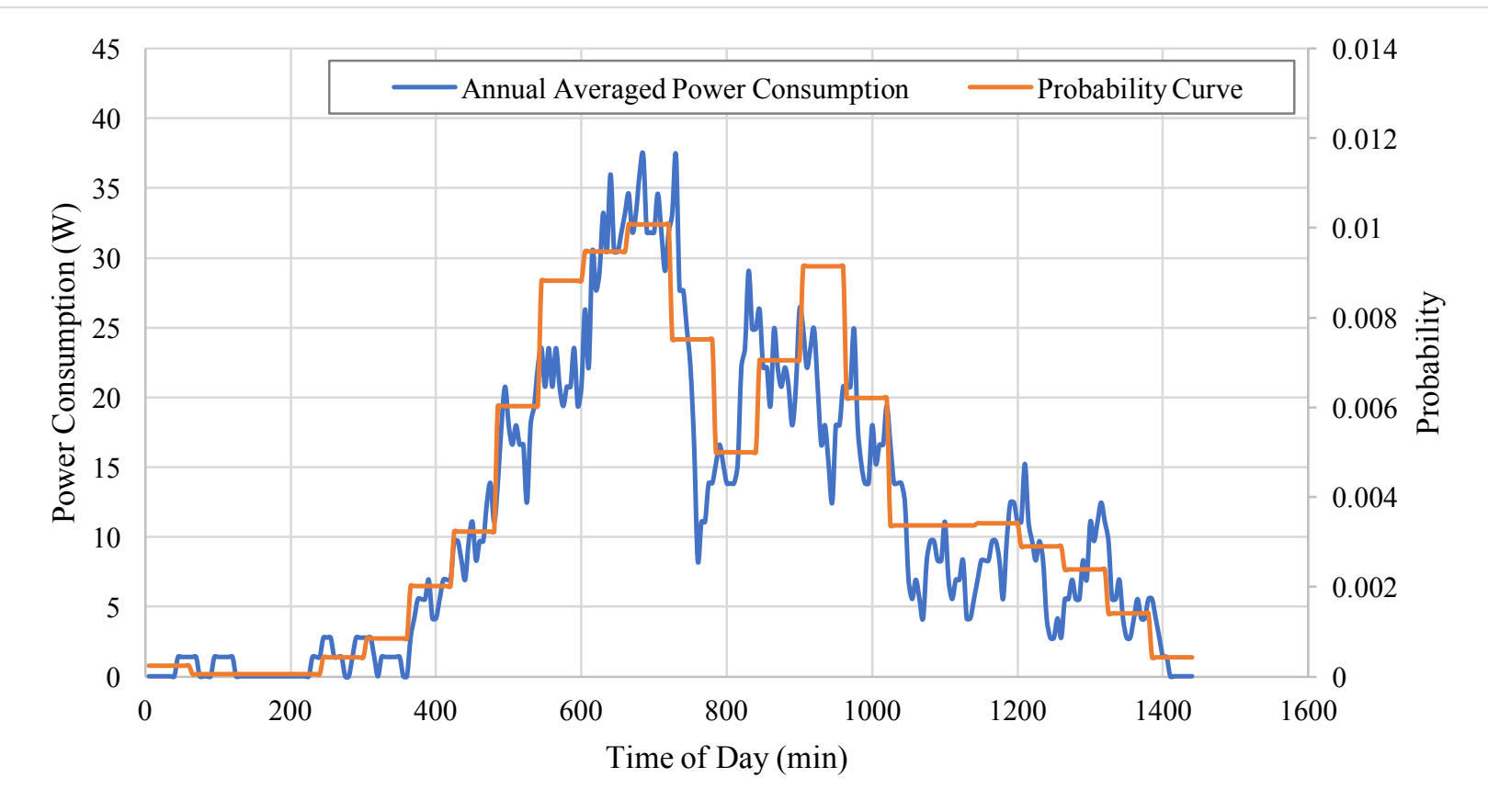

Figure 4-24: Sample clothes washer daily averaged load profile and probability curve (medium energy house) 


\subsubsection{Clothes Dryer}

The dryer power consumption was simulated based on its probability curve. Cycle times were randomly chosen using a uniform distribution between $30-60$ minutes. The results of the calibration procedure are summarized in Table 4-19, while Figure 4-25 displays a sample daily averaged load profile. Any sequential operation between the clothes washer and dryer operation is ignored, and the dryer's operation is simulated only using the probability curve.

Table 4-19: Clothes dryer simulation performance

\begin{tabular}{|c|c|c|c|c|c|}
\hline & \multicolumn{2}{|c|}{$\begin{array}{c}\text { Annual energy consumption } \\
(\mathbf{k W h} / \mathbf{y r})\end{array}$} & Total number of cycles per year & \\
\hline $\begin{array}{c}\text { House } \\
\text { type }\end{array}$ & Target & Simulated & Target & Simulated & $\begin{array}{c}\text { Chance } \\
\text { Factor }\end{array}$ \\
\hline Low & 593 & $\begin{array}{c}591.1 \\
(\sigma=45.3, \mathrm{n}=30)\end{array}$ & 192 & $\begin{array}{c}191 \\
(\sigma=13.3, \mathrm{n}=30)\end{array}$ & 22.3 \\
\hline Medium & 1284 & $\begin{array}{c}1294.8 \\
(\sigma=54.9, \mathrm{n}=30)\end{array}$ & 416 & $\begin{array}{c}419 \\
(\sigma=16.8, \mathrm{n}=30)\end{array}$ & 9.8 \\
\hline High & 1976 & $\begin{array}{c}1970.9 \\
(\sigma=71.7, \mathrm{n}=30)\end{array}$ & 640 & $\begin{array}{c}639 \\
(\sigma=21.9, \mathrm{n}=30)\end{array}$ & 6.1 \\
\hline
\end{tabular}

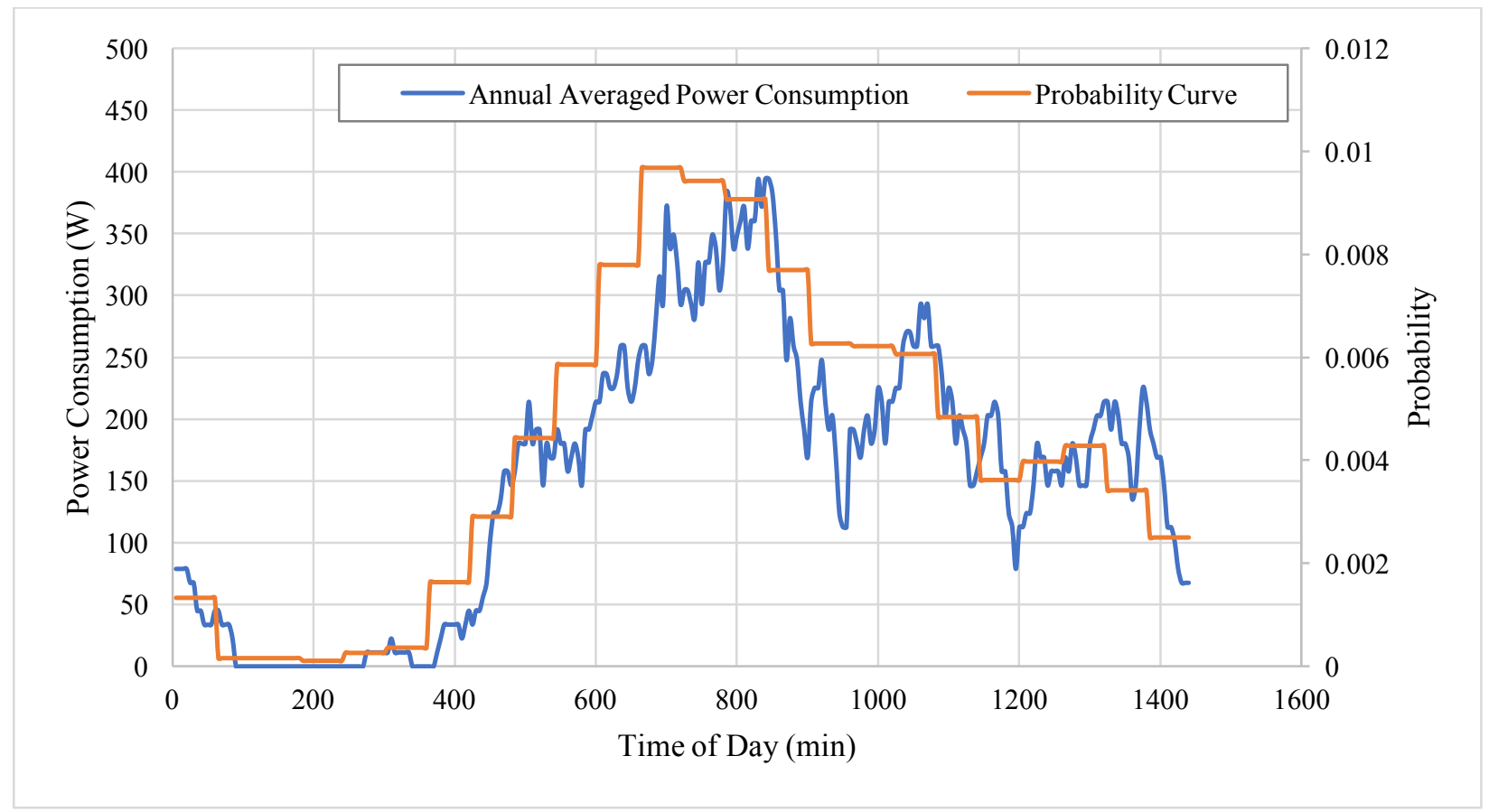

Figure 4-25: Sample clothes dryer daily averaged load profile and probability curve (medium energy house) 


\subsubsection{Range}

The range (including both the stove-top and oven) was simulated entirely based on its probability curve. Cycle times were randomly generated using a uniform distribution between $15-70$ minutes. The performance of the range simulation is summarized in Table 4-20, while a sample daily averaged load profile is displayed in Figure 4-26.

Table 4-20: Range simulation performance

\begin{tabular}{|c|c|c|c|c|c|}
\hline & \multicolumn{2}{|c|}{$\begin{array}{c}\text { Annual energy consumption } \\
\mathbf{( k W h} / \mathbf{y r})\end{array}$} & Total number of cycles per year & \\
\hline $\begin{array}{c}\text { House } \\
\text { type }\end{array}$ & Target & Simulated & Target & Simulated & $\begin{array}{c}\text { Chance } \\
\text { Factor }\end{array}$ \\
\hline $\begin{array}{c}\text { Low and } \\
\text { Medium }\end{array}$ & 769 & $\begin{array}{c}765.2 \\
(\sigma=29.2, \mathrm{n}=30)\end{array}$ & 678 & $\begin{array}{c}677 \\
(\sigma=24.8, \mathrm{n}=30)\end{array}$ & 5.9 \\
\hline High & 1077 & $\begin{array}{c}1078.2 \\
(\sigma=32.2, \mathrm{n}=30)\end{array}$ & 950 & $\begin{array}{c}951 \\
(\sigma=23.7, \mathrm{n}=30)\end{array}$ & 4.1 \\
\hline
\end{tabular}

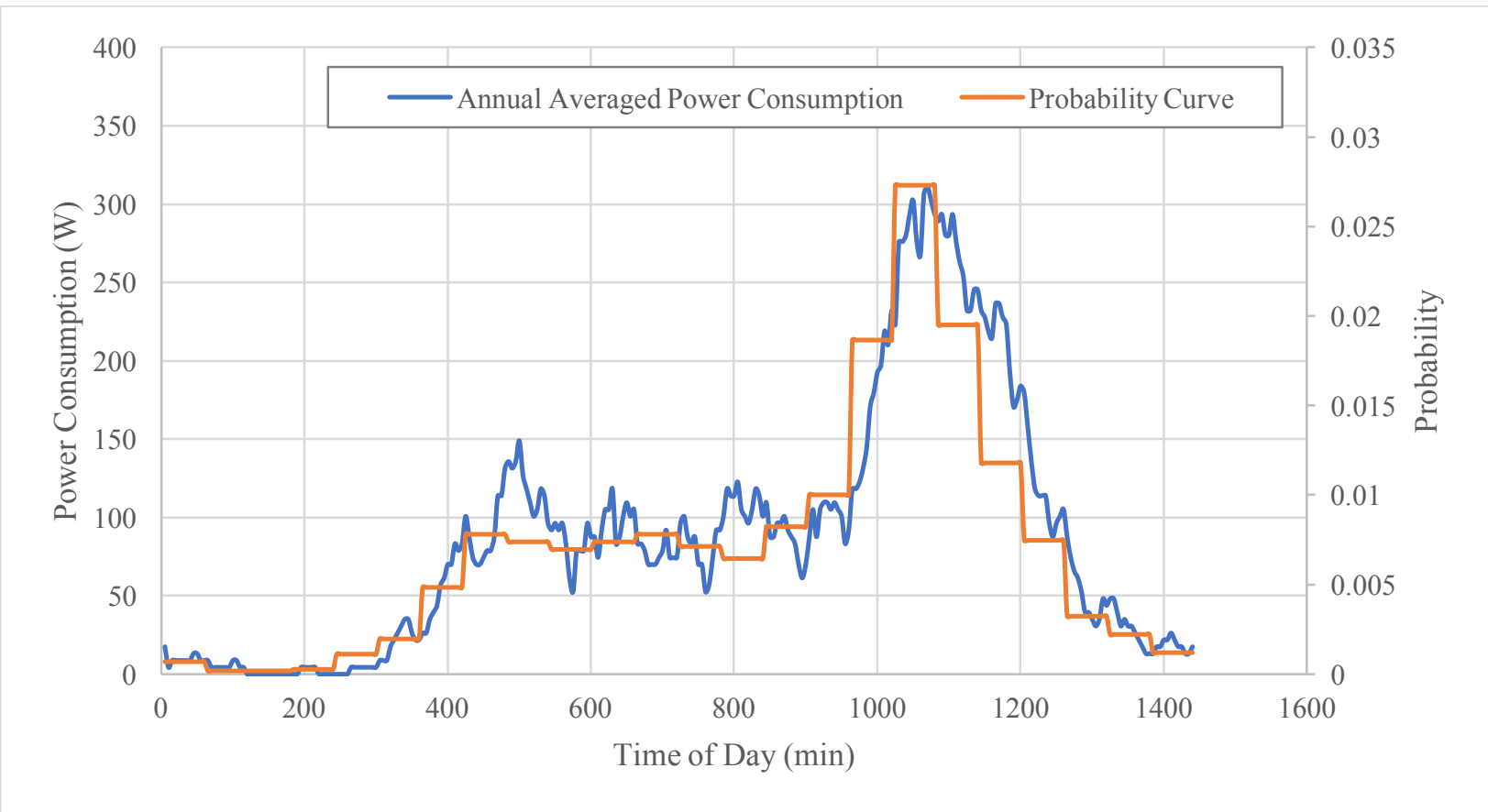

Figure 4-26: Sample range daily averaged load profile and probability curve (medium energy house) 


\subsubsection{Miscellaneous Appliances}

Numerous small appliances are grouped into the miscellaneous category. A selection of small appliances was chosen from the available data, summarized in Table 4-21. To calculate the miscellaneous appliance load, each individual appliance was simulated separately, and then the power consumption was aggregated into a single load profile.

Table 4-21: List of miscellaneous appliances considered for simulation (U.S. Department of Energy, 2011)

\begin{tabular}{|l|c|c|c|c|}
\hline \multicolumn{1}{|c|}{ Appliance } & $\begin{array}{c}\text { Operating } \\
\text { power (W) }\end{array}$ & $\begin{array}{c}\text { Standby power } \\
\text { (off) (W) }\end{array}$ & $\begin{array}{c}\text { Operating } \\
\text { hours per day }\end{array}$ & $\begin{array}{c}\text { Units per } \\
\text { house }\end{array}$ \\
\hline First TV & 110 & 4 & 7.1 & 0.89 \\
\hline Second TV & 92 & 4 & 4.2 & 0.61 \\
\hline Radio & 2 & 1 & 0.96 & 0.49 \\
\hline Cable Box & 16 & 15 & 7.48 & 0.57 \\
\hline Laptop PC (Plugged In) & 25 & 2 & 6.5 & 0.28 \\
\hline Desktop PC w/ Speakers & 75 & 2 & 8.1 & 0.91 \\
\hline Printer (Inkjet) & 8.9 & 1.7 & 0.24 & 0.66 \\
\hline Microwave & 1500 & 3 & 0.19 & 0.88 \\
\hline Coffee Maker & 1100 & 0.4 & 0.10 & 0.61 \\
\hline Toaster Oven & 1300 & 0.82 & 0.07 & 0.34 \\
\hline Toaster & 1050 & 0.82 & 0.10 & 0.90 \\
\hline Blender & 192 & 0 & 0.10 & 0.79 \\
\hline Hand Mixer & 55 & 0 & 0.10 & 0.88 \\
\hline Hair Dryer & 1500 & 0 & 0.06 & 0.86 \\
\hline Clock Radio & 2 & 1.7 & 0.24 & 1.35 \\
\hline Cell Phone Charger & 2.6 & 0.3 & 1.2 & 1.74 \\
\hline Fan (Portable) & 31 & 0 & 1.0 & 0.95 \\
\hline Iron & 1350 & 0 & 0.11 & 0.92 \\
\hline Vacuum Cleaner (Upright) & 1080 & 0 & 0.11 & 0.98 \\
\hline
\end{tabular}

The standard miscellaneous probability curve was multiplied by both the operating hours per day, and the units per house, such that the shape of the probability curve would be the same for each small appliance, but they would be weighted appropriately against each other. The same chance factor was applied to each of the individual TOU curves, and the same procedure used to calibrate the other appliances was implemented. The number of cycles for each miscellaneous appliance 
was ignored. Table 4-22 summarizes the miscellaneous appliance simulation performance, while Figure 4-27 shows a sample daily averaged load profile.

Table 4-22: Miscellaneous appliance simulation performance

\begin{tabular}{|c|c|c|c|}
\hline & \multicolumn{2}{|c|}{$\begin{array}{c}\text { Annual energy consumption } \\
(\mathbf{k W h} / \mathbf{y r})\end{array}$} & \\
\hline $\begin{array}{c}\text { House } \\
\text { type }\end{array}$ & Target & Simulated & $\begin{array}{c}\text { Chance } \\
\text { Factor }\end{array}$ \\
\hline Low & 1516.8 & $\begin{array}{c}1523.6 \\
(\sigma=26.5, \mathrm{n}=30)\end{array}$ & 13.7 \\
\hline Medium & 2464.8 & $\begin{array}{c}2453.7 \\
(\sigma=37.5, \mathrm{n}=30)\end{array}$ & 6.6 \\
\hline High & 3223.2 & $\begin{array}{c}3222.4 \\
(\sigma=44.5, \mathrm{n}=30)\end{array}$ & 4.4 \\
\hline
\end{tabular}

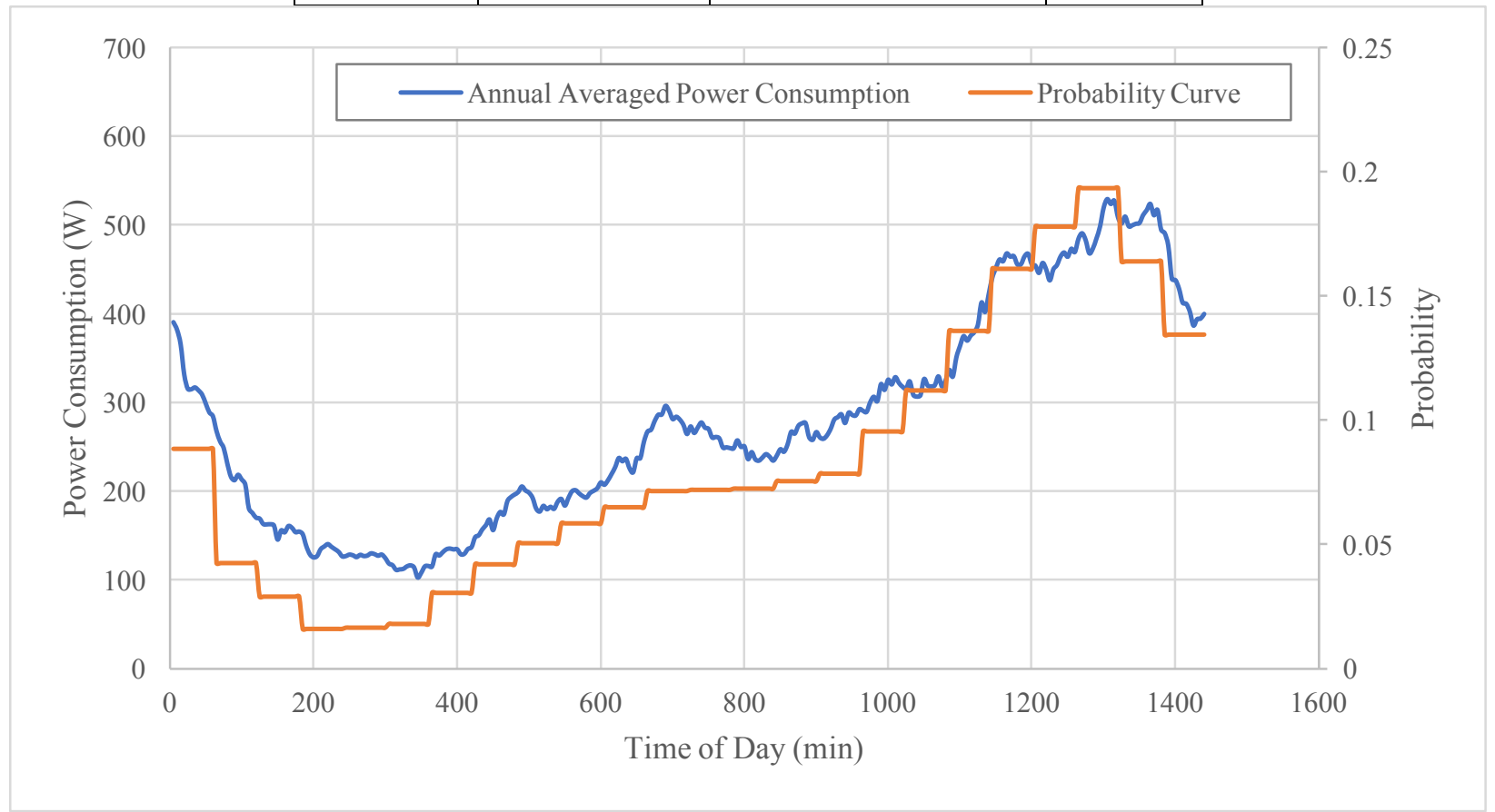

Figure 4-27: Sample aggregated miscellaneous appliance daily averaged load profile and probability curve (medium energy house)

\subsubsection{Lighting}

The lighting in the home was split into 5 separate lighting loads for each of the three house types, summarized in Table 4-23. The lighting TOU curves reflected seasonal changes and four different 
curves were used, one for each season. Additional space heating associated with lighting has been neglected.

Table 4-23: Lighting load summary (Armstrong, Swinton, Ribberink, Beausoleil-Morrison, \& Millette, 2009)

\begin{tabular}{|l|l|l|l|}
\hline & $\begin{array}{l}\text { Low } \\
\text { energy }\end{array}$ & $\begin{array}{l}\text { Medium } \\
\text { energy }\end{array}$ & $\begin{array}{l}\text { High } \\
\text { energy }\end{array}$ \\
\hline Lighting load 1 (W) & 30 & 60 & 120 \\
\hline Lighting load 2 (W) & 50 & 100 & 200 \\
\hline Lighting load 3(W) & 60 & 120 & 240 \\
\hline Lighting load 4 (W) & 205 & 410 & 820 \\
\hline Lighting load 5(W) & 100 & 200 & 400 \\
\hline Target Consumption (kWh/yr) & $\mathbf{1 0 1 5}$ & $\mathbf{2 0 3 0}$ & $\mathbf{4 0 6 1}$ \\
\hline
\end{tabular}

The performance of the lighting load simulation is shown in Table 4-24. Each of the cases uses the same chance factor, since the annual targets are simply linearly scaled. A sample daily averaged lighting load profile is shown in Figure 4-28.

Table 4-24: Lighting load simulation performance

\begin{tabular}{|c|c|c|c|}
\hline & \multicolumn{2}{|c|}{$\begin{array}{c}\text { Annual energy consumption } \\
(\mathbf{k W h} / \mathbf{y r})\end{array}$} & \\
\hline $\begin{array}{c}\text { House } \\
\text { type }\end{array}$ & Target & Simulated & $\begin{array}{c}\text { Chance } \\
\text { Factor }\end{array}$ \\
\hline Low & 1015 & $\begin{array}{c}1016.9 \\
(\sigma=9.4, \mathrm{n}=30)\end{array}$ & 1.3 \\
\hline Medium & 2030 & $\begin{array}{c}2041.2 \\
(\sigma=21.9, \mathrm{n}=30)\end{array}$ & 1.3 \\
\hline High & 4061 & $\begin{array}{c}4082.4 \\
(\sigma=30.7, \mathrm{n}=30)\end{array}$ & 1.3 \\
\hline
\end{tabular}




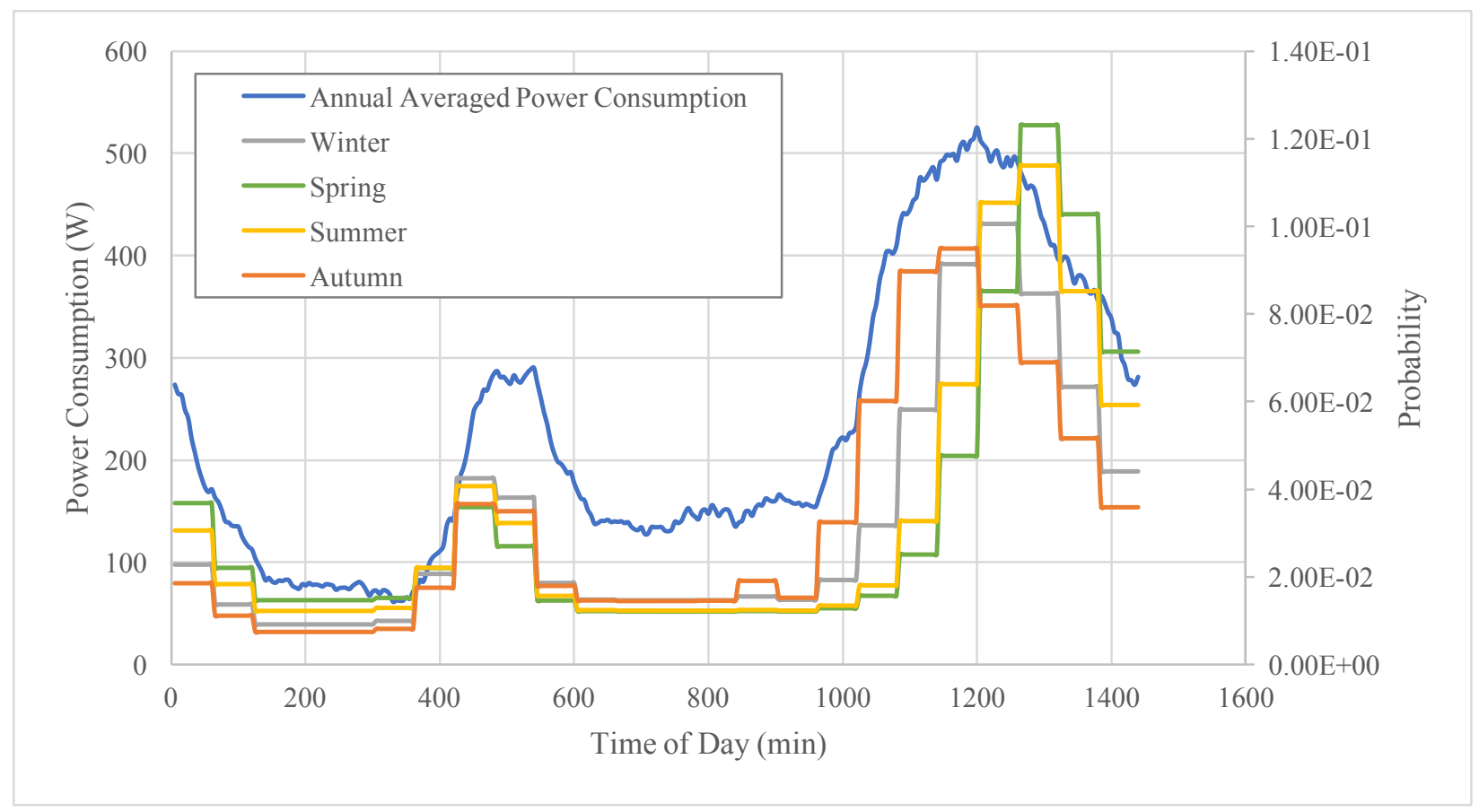

Figure 4-28: Sample lighting load daily averaged load profile and probability curve (medium energy house)

Thirty distinct simulated load profiles for each of the eight appliances were generated and saved for each of the three house types. These were then aggregated into 30 load profiles for each of the low, medium, and high energy consuming households, totaling in 90 different non-HVAC load profiles. Each of these profiles can then be combined with the electrical loads calculated from the HVAC system models in order to reflect the variability and randomness on the demand side of the system. 


\section{Experimental Setup}

Two main experiments were performed to verify specific components of the EMSs investigated in this work. The first experiment investigated the practicalities behind load shifting, an example of a deterministic EMS, while the second examined the communication protocol behind a transactive energy system, an example of an adaptive EMS.

\subsection{Load Shifting Experiment}

The load shifting experiment had two main goals. The first was to experimentally implement a load shifting procedure using all real-world devices. The second goal was to do so through the development of software with a general architecture, such that it might be applied to future studies utilizing more advanced EMS. The configuration of the components used in the experimental load shifting EMS are shown in Figure 5-1. For this experiment, the basic load shifting procedure described in Section 3.2.1 was performed on the ASHP in House A using the 1600 Ah lead-acid battery bank. Since the full $1600 \mathrm{Ah}(\sim 75 \mathrm{kWh})$ capacity is unrealistic for a conventional residential building, a $25 \mathrm{kWh}$ limit was imposed, to represent a more realistic residential battery bank. The $4.08 \mathrm{~kW}$ roof-mounted PV and $2.4 \mathrm{~kW}$ wind turbines were used as the zero-feedback DGs under test. A Conext ComBox was used in order to monitor battery parameters and implement control actions. Communication with the ComBox was enabled through Modbus protocol.

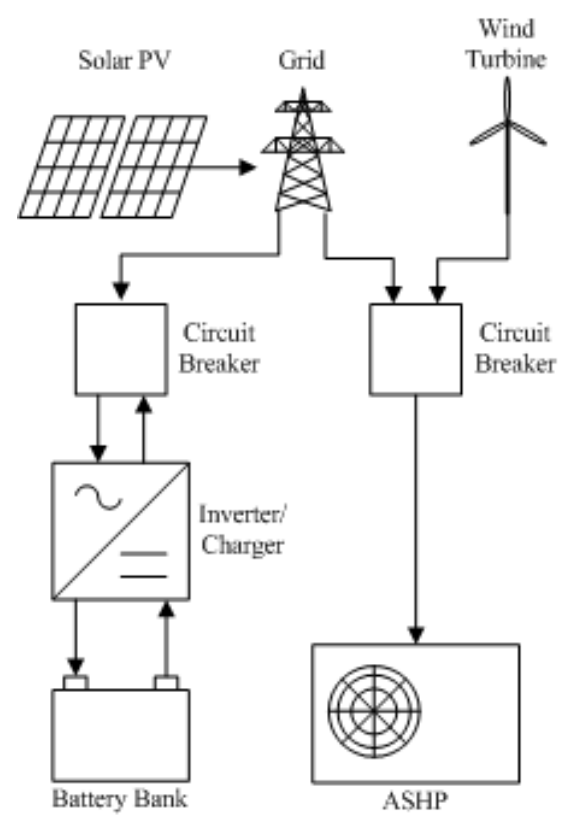

Figure 5-1: Diagram of experimental load shifting setup 
All software was developed and implemented using LabVIEW. A state diagram of the software is pictured in Figure 5-2. The existing DMS was utilized to monitor the energy generation of the PV and wind turbine systems, along with the power consumption of the ASHP. The LabVIEW controller used Modbus to communicate and control the battery bank via the Combox and inverter/charger. This allowed specific battery parameters to be read through LabVIEW, and allowed certain control parameters to be written to the inverter/charger itself. The Combox allowed the exact rate of battery discharge to be set to the nearest $0.1 \mathrm{~A}$. The charging rate was fixed at the maximum $6 \mathrm{~kW}$ capacity of the invertor/charger, and charging was only performed overnight. A strict charging process was obeyed in order to preserve the lifespan of the battery bank.

For simplicity, an open-loop controller was initially developed to control the battery output current. It was determined that, although typically not as accurate as its closed-loop alternative, it was acceptable for this application since the battery output current could be communicated directly to the inverter via the control software. In later iterations of the control software, a closed loop PIcontroller was implemented with similar accuracy to the open-loop controller.

The battery bank was used to match the net load between the ASHP, PV, and wind turbine, if the following three conditions were met:

1. The hour was between 7:00 and 19:00,

2. The output required by the battery was less than the output capacity of the inverter $(6 \mathrm{~kW})$, and,

3. The battery had not yet expended all of its useful energy $(25 \mathrm{kWh})$ within its current discharge cycle.

An initial goal of this study was to implement PID control over the ASHP to minimize energy consumption, while still maintaining thermal comfort. Unfortunately, throughout the course of the experiment it was determined that the ASHP model under test did not allow for this control capability; only ON/OFF control could be implemented programmatically over the ASHP. However, the variable capacity ASHP continuously adjusted its output to meet the building load. 
Therefore, the ASHP was running part-load for most of the heating season, except during the extremely cold outdoor temperature of $-15^{\circ} \mathrm{C}$ or below.

In order to try to reduce the power consumption of the ASHP further, thus reducing strain on the battery bank, a temperature setback was implemented during some phases of the experiment. This setback involved reducing the set point of House $\mathrm{A}$ to $18^{\circ} \mathrm{C}$ between the hours of 10:00 - 17:00, under the assumption that homeowners would be away at work during these hours.

A major assumption for all EMS simulations is that the battery bank was capable of actively tracking a load by matching the load's power consumption with its output. This experiment was designed to show that not only is this possible, but it is achievable using simple, non-specialized components. 


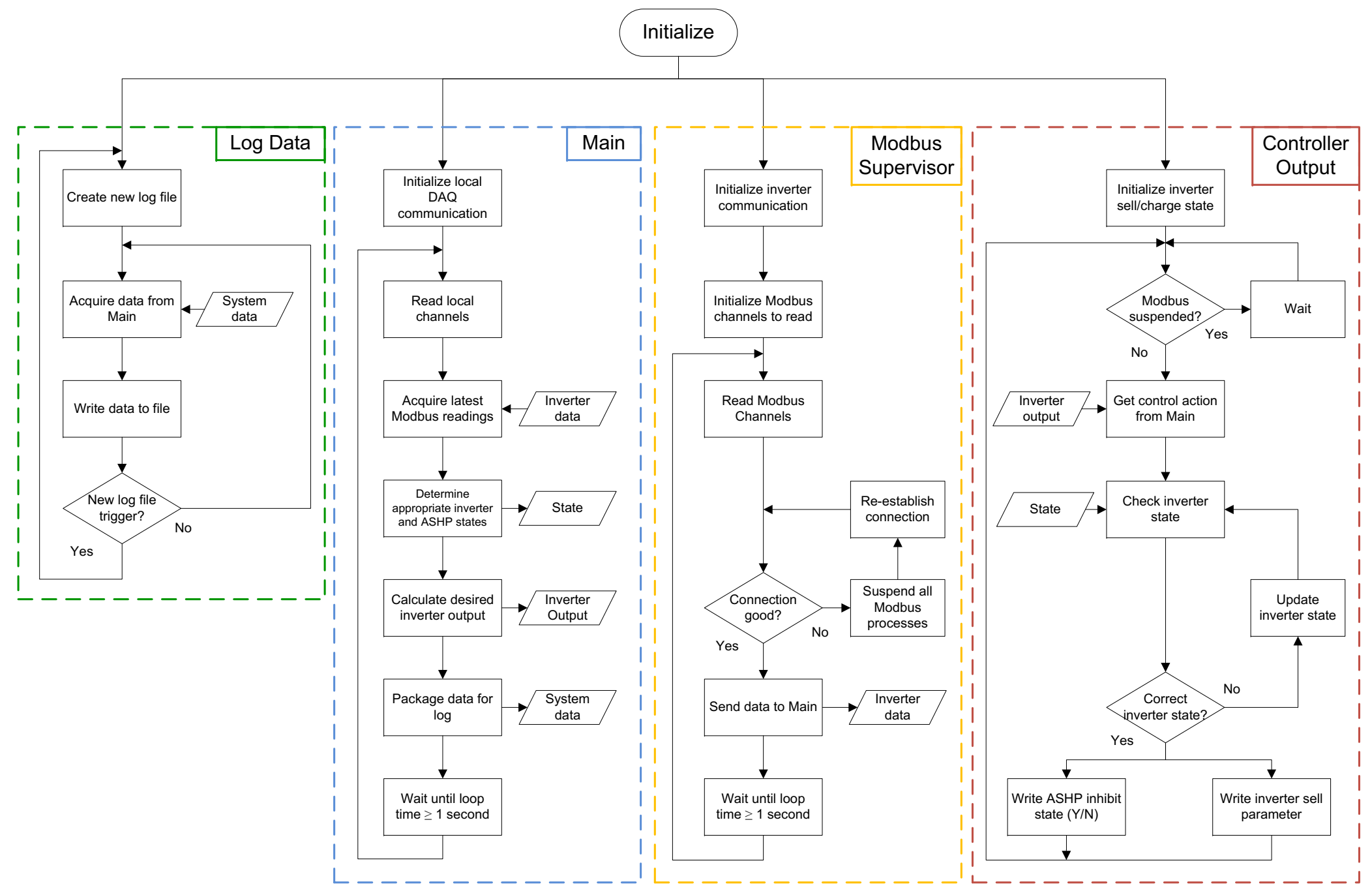

Figure 5-2: State diagram of battery control program 


\subsection{Transactive Energy Management System Demonstration}

A demonstration of the TEMS was performed at the ASH. The purpose of the demonstration was not to show the full potential of the system, but rather act simply as a proof of concept, to show that different devices could communicate and transactional-based decisions could be made in real time. The simplified system consisted of one flexible load, the HPWH in House B, and one renewable generation source, the $4.08 \mathrm{kWp}$ roof-mounted solar PV. The existing DMS was integrated into the Marketplace for monitoring purposes.

The demonstration brought the MATLAB Energy Marketplace into a LabVIEW environment, and operated using the RTI Connext Data Distribution Service (DDS) communications framework. DDS is an open-source middleware framework that facilitates real-time, secure and reliable communication within distributed systems. It handles the sending, receiving, transportation and identification of data from the user, allowing the development of highly scalable systems. DDS uses a topic-based publish/subscribe approach. Simply put, this means that different agents can either write to (publish) or read from (subscribe) different data streams (topics). They can also both publish and subscribe to the same topic. Agents are not the devices themselves, but the smart communication devices that facilitate the entry of an energy consuming or producing device into the marketplace.

The load agent used in the demonstration was the HPWH. This agent utilized a bid curve, pictured in Figure 5-3, that was a function of water tank temperature at the outlet of the tank. The bid curve was designed to pay for solar energy up until it was $2^{\circ} \mathrm{C}$ above the tank's set point, and only pay for on-peak electricity when the water temperature fell to $5^{\circ} \mathrm{C}$ below the set point. 


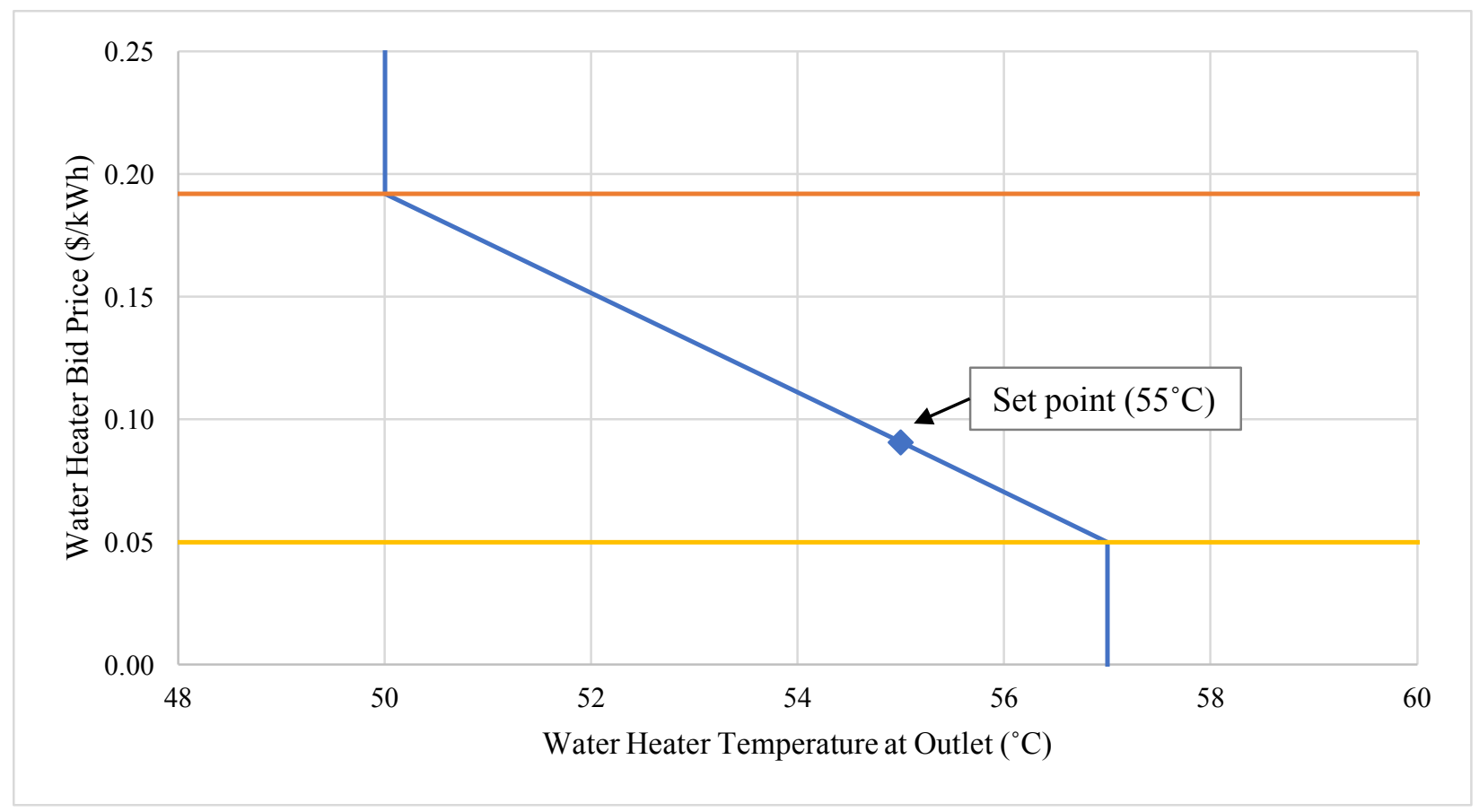

Figure 5-3: HPWH bid curve for Marketplace demonstration

Water temperature was measured using an RTD-122 sensor submerged in the tank itself at the level of the hot water outlet. Power consumption of the water heater was monitored using a WattNode pulse meter.

The water heater was turned on and off using a digital relay that controlled a SafePlug Wired Load Controller that the water heater was connected to. While some SafePlug devices allow open communication and control, this particular model did not allow programmatic control, hence the use of the digital relay. The water heater agent would monitor the power consumption of the HPWH in order to ensure that it was off or on when it should have been. If the Marketplace did not grant the bid of the water heater, then the relay forced the SafePlug off, which turned off the water heater. This solution would not be suitable for long-term operation, however for the short demonstration performed it sufficed.

The solar PV agent bid a fixed bid price of $5 \notin / \mathrm{kWh}$ for all energy. Its power production was also measured using a WattNode pulse meter. The solar PV agent was merely a monitoring agent that sent bids to the marketplace, as no control actions were applied to the modules' inverter. 
The main aim of the demonstration is to showcase that, by using the DDS communication protocol, agents can be added or removed from the TEMS at will, without halting system operation or the need for modifications to system design. If this proves to be possible it will enforce the scalability of the system, and its inherent plug-and-play nature. 


\section{Simulation Results and Discussion}

This Chapter details the results of the EMSs described in Chapter 3, on the simulated performance of the house model developed in Chapter 4. Additional sensitivity analyses regarding the economics and emissions associated with some of the discussed EMSs are presented in Chapter 8.

\subsection{Baseline Scenarios}

\subsubsection{Space Conditioning Energy Consumption}

Table 6-1 summarizes the simulated ASHP energy provided, electricity consumption, and seasonal COP. These values are in close agreement with previous simulation work conducted on this same system (Safa, Fung, \& Kumar, 2015), and will be used as the baseline for all comparisons. The energy consumed by the AHU and the HRV is summarized in Table 6-2.

Table 6-1: Seasonal simulated heating and cooling energy consumption-Base case

\begin{tabular}{|l|c|c|c|}
\hline \multicolumn{1}{|c|}{ Season } & $\begin{array}{c}\text { Heating or cooling } \\
\text { provided }(\mathrm{kWh})\end{array}$ & $\begin{array}{c}\text { Electricity } \\
\text { consumption }(\mathrm{kWh})\end{array}$ & Seasonal COP \\
\hline Heating & 16,174 & 4,744 & 3.41 \\
\hline Cooling & 2,295 & 439 & 5.23 \\
\hline
\end{tabular}

Table 6-2: AHU and HRV electricity consumption summary

\begin{tabular}{|l|c|c|}
\hline \multicolumn{1}{|c|}{ Season } & $\begin{array}{c}\text { AHU electricity } \\
\text { consumption }(\mathrm{kWh})\end{array}$ & $\begin{array}{c}\text { HRV electricity } \\
\text { consumption }(\mathrm{kWh})\end{array}$ \\
\hline Heating & 1,672 & 2,416 \\
\hline Cooling & 873 & 1,368 \\
\hline Annual & 2,545 & 3,784 \\
\hline
\end{tabular}

\subsubsection{Domestic Hot Water Energy Consumption}

\subsubsection{Electric Mode}

The annual energy consumption of the simulated water heater in electric mode compared to its target is displayed in Table 6-3. These results show good agreement with the target values. Unless otherwise indicated, the water tank will be assumed to operate in electric mode, and not heat pump mode. The target DHW energy consumption was obtained from the Department of Energy, 
because energy consumption values could be obtained under the exact conditions of this simulation work.

Table 6-3: DHW electric mode simulation performance

\begin{tabular}{|c|c|c|}
\hline $\begin{array}{c}\text { Simulated DHW energy } \\
\text { consumption }(\mathrm{kWh} / \text { year })\end{array}$ & $\begin{array}{c}\text { Target DHW energy } \\
\text { consumption }(\mathrm{kWh} / \text { year })^{\mathrm{a}}\end{array}$ & Percent difference \\
\hline 4088 & $3,990^{\mathrm{a}}$ & $+2.46 \%$ \\
\hline
\end{tabular}

a. (Department of Energy, 2017)

\subsubsection{Heat Pump Mode}

The annual energy consumption of the simulated water heater in heat pump mode compared to its target is displayed in Table 6-4. These results are in good agreement with the previous work (Amirirad, 2016).

Table 6-4: DHW heat pump mode simulation performance

\begin{tabular}{|c|c|c|}
\hline $\begin{array}{c}\text { Simulated DHW energy } \\
\text { consumption }(\mathrm{kWh} / \text { year })\end{array}$ & $\begin{array}{c}\text { Target DHW energy } \\
\text { consumption }(\mathrm{kWh} / \text { year })^{\mathrm{a}}\end{array}$ & Percent difference \\
\hline 1,626 & $1,639^{\mathrm{a}}$ & $-0.79 \%$ \\
\hline
\end{tabular}

a. (Amirirad, 2016)

When operating in heat pump mode, the impact on the space heating and cooling load are also of interest. Table 6-5 summarizes the changes in heating and cooling energy consumption, associated with running the water heater in heat pump mode. As expected, a decrease in space cooling and increase in space heating can be seen.

Table 6-5: Changes in space conditioning electricity consumption when operating the water heater in heat pump mode

\begin{tabular}{|c|c|c|c|c|}
\hline & Season & $\begin{array}{c}\text { DHW - Electric } \\
\text { mode } \mathbf{( k W h )}\end{array}$ & $\begin{array}{c}\text { DHW - Heat pump } \\
\text { mode (kWh) }\end{array}$ & Change \\
\hline \multirow{3}{*}{ Space conditioning } & Heating & 4744 & 5098.6 & $7.5 \%$ \\
\cline { 2 - 5 } & Cooling & 439 & 349.6 & $-20.4 \%$ \\
\cline { 2 - 5 } & Annual & 5183 & 5448.2 & $5.1 \%$ \\
\hline \multirow{3}{*}{ DHW } & Heating & 2843 & 1130 & $-60.3 \%$ \\
\cline { 2 - 5 } & Cooling & 1245 & 496 & $-60.2 \%$ \\
\cline { 2 - 5 } & Annual & 4088 & 1626 & $-60.2 \%$ \\
\hline \multicolumn{2}{|c|}{ Total annual consumption } & $\mathbf{9 2 7 1}$ & $\mathbf{7 0 7 4 . 2}$ & $\mathbf{- 2 3 . 7 \%}$ \\
\hline
\end{tabular}




\subsubsection{Non-HVAC Load Energy Consumption}

Using the stochastic non-HVAC load simulation procedure, 30 distinct load profiles were generated for each of the three house types (low, medium, and high energy consumption), totaling 90 load profiles. This number was chosen arbitrarily to represent sufficient demand variability. Figure 6-1 displays a sample profile from each house type. The profiles displayed show the power consumption from all non-HVAC loads averaged by hour from one annual data set from each of the three house types. Table 6-6 summarizes the simulated annual consumption compared to target values.

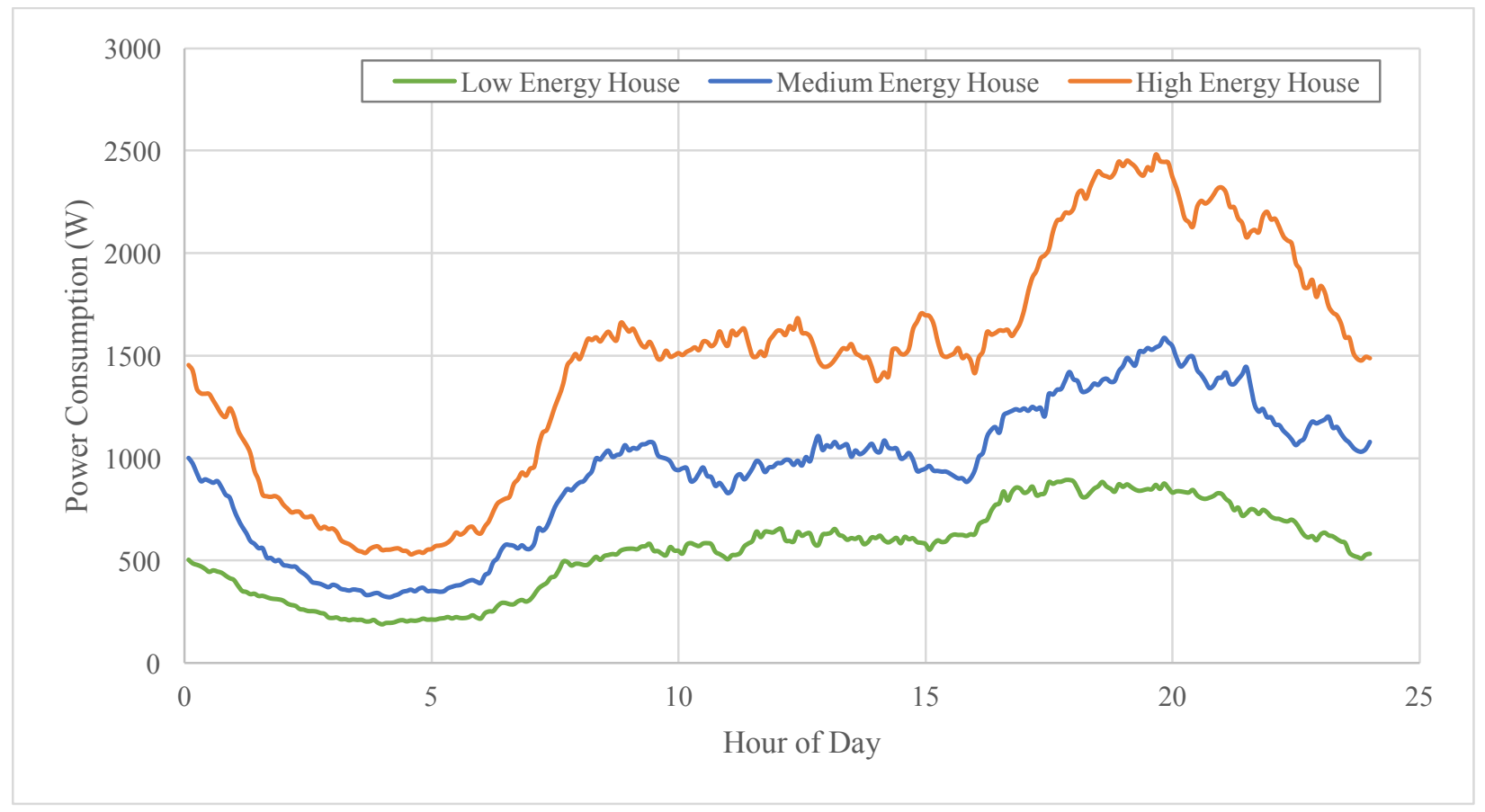

Figure 6-1: Sample hourly averaged simulated non-HVAC load profiles

Table 6-6: Non-HVAC load simulation performance

\begin{tabular}{|l|c|c|c|}
\hline \multicolumn{1}{|c|}{ House type } & $\begin{array}{c}\text { Simulated average annual } \\
\text { consumption }(\mathrm{kWh})\end{array}$ & $\begin{array}{c}\text { Annual consumption target } \\
(\mathrm{kWh})^{\mathrm{a}}\end{array}$ & $\begin{array}{c}\text { Percent } \\
\text { difference }\end{array}$ \\
\hline Low energy & $4,815(\sigma=55.4, \mathrm{n}=30)$ & 4,813 & $+0.04 \%$ \\
\hline Medium energy & $8,149(\sigma=82.8, \mathrm{n}=30)$ & 8,156 & $-0.09 \%$ \\
\hline High energy & $12,993(\sigma=108.8, \mathrm{n}=30)$ & 13,011 & $-0.14 \%$ \\
\hline
\end{tabular}

a. (Armstrong, Swinton, Ribberink, Beausoleil-Morrison, \& Millette, 2009) 


\subsubsection{Total Baseline Energy Consumption}

Accounting for all sources of energy in a typical residential home, the final simulated energy consumption for each house type is summarized in Table 6-7, while Figure 6-2 illustrates this same summary in terms of the overall percentage of energy end-use. The energy consumed by both the AHU and HRV has been added the space heating and space cooling consumption based on its season of operation.

Table 6-7: Total simulated energy end-use summary

\begin{tabular}{|l|c|c|c|}
\hline \multicolumn{1}{|c|}{ Energy End-Use } & $\begin{array}{c}\text { Low Energy House } \\
\text { (kWh/year) }\end{array}$ & $\begin{array}{c}\text { Medium Energy } \\
\text { House (kWh/year) }\end{array}$ & $\begin{array}{c}\text { High Energy House } \\
\text { (kWh/year) }\end{array}$ \\
\hline Space heating & 8,832 & 8,832 & 8,832 \\
\hline Water heating & 4,088 & 4,088 & 4,088 \\
\hline Appliances & 3,798 & 6,108 & 8,911 \\
\hline Lighting & 1,017 & 2,041 & 4,082 \\
\hline Space cooling & 2,681 & 2,681 & 2,681 \\
\hline $\begin{array}{l}\text { Total Annual } \\
\text { Consumption }\end{array}$ & $\mathbf{2 0 , 4 1 6}$ & $\mathbf{2 3 , 7 5 0}$ & $\mathbf{2 8 , 5 9 4}$ \\
\hline
\end{tabular}

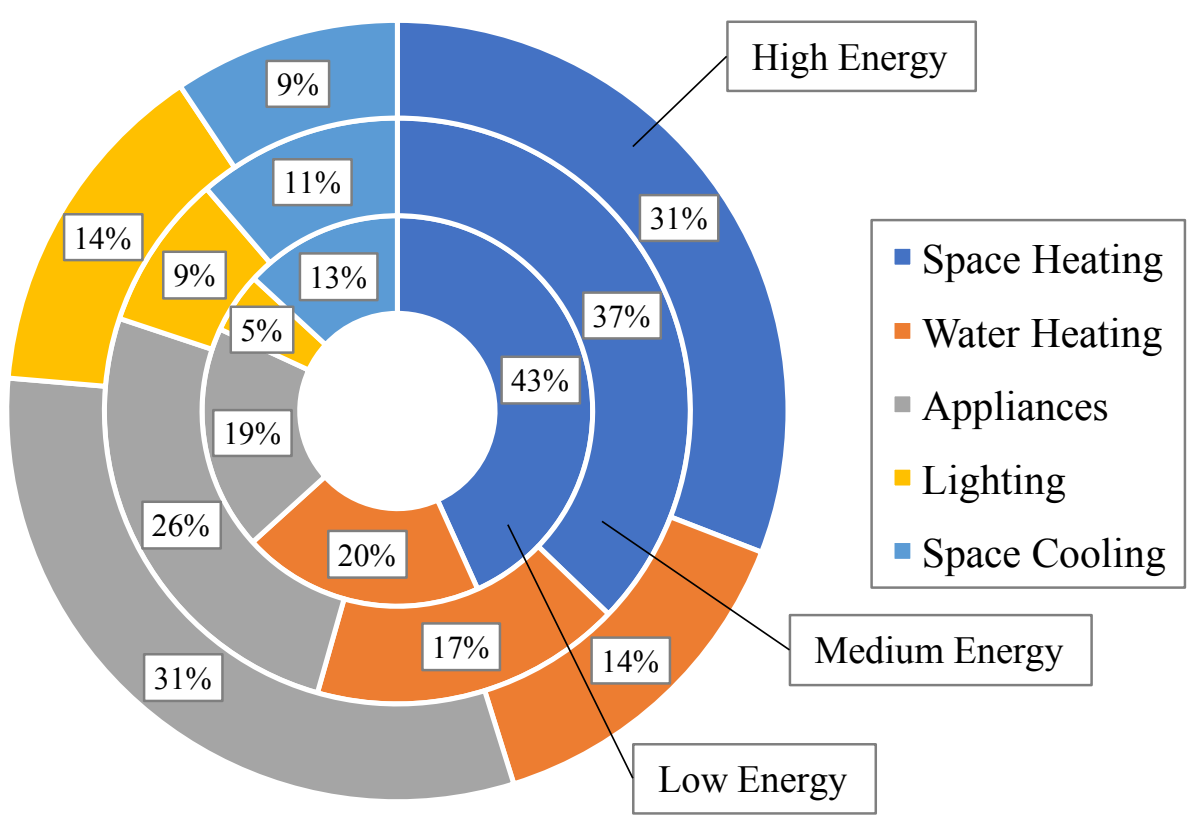

Figure 6-2: Total simulated energy end-use percentage breakdown 
Figure 6-3 summarizes the energy consumption distribution over the three TOU pricing periods. Since appliance usage is the only variable between the three different cases, and the general shape of the appliance load curves is the same for all three cases, the distribution across TOU periods is nearly identical. For all other cases, only the medium consumption home's distribution will be shown, unless there is a notable difference between building types.

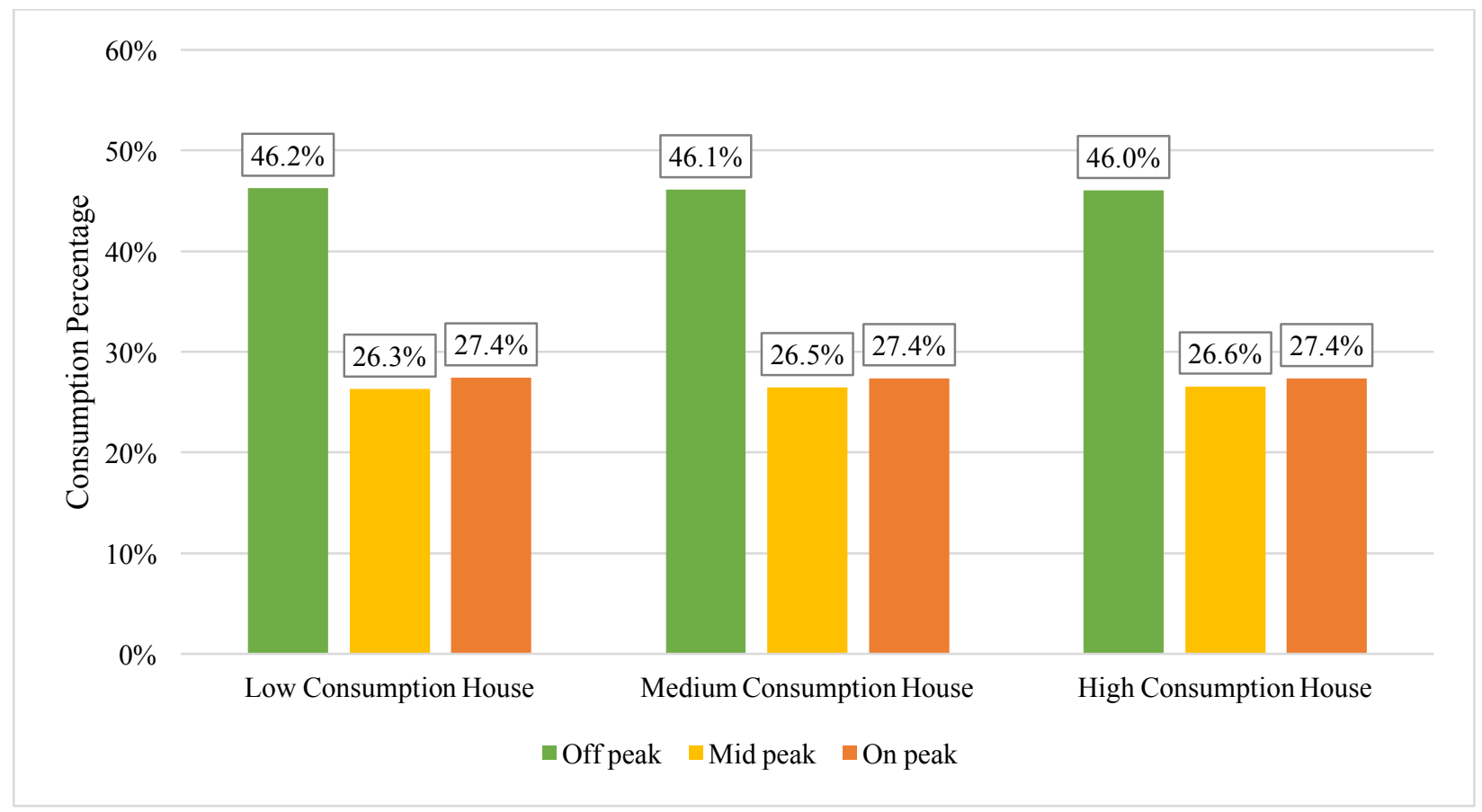

Figure 6-3: TOU consumption distribution for normal house operation

\subsubsection{Renewable Energy Generation}

The models described in Section 4.8 were used to simulate all renewable energy production for the year. Their annual outputs are summarized in Table 6-8. Different combinations of the included renewables have been investigated via simulation, in order to represent residential buildings with different system sizes and configurations.

Table 6-8: Annual renewable generation summary

\begin{tabular}{|l|c|}
\hline \multicolumn{1}{|c|}{ System } & $\begin{array}{c}\text { Annual generation } \\
(\mathrm{kWh})\end{array}$ \\
\hline Roof-mounted solar PV (5 kW) & 5,585 \\
\hline Skystream 2.4 kW wind turbine & 338 \\
\hline Bergey 6 kW wind turbine & 1,261 \\
\hline Bergey 10 kW wind turbine & 1,507 \\
\hline
\end{tabular}


Figure 6-4 displays the TOU consumption distribution when using two renewable energy zerofeedback systems. A clear decrease in mid- and on-peak consumption can be attributed primarily to on-site solar energy utilization at those times of day.

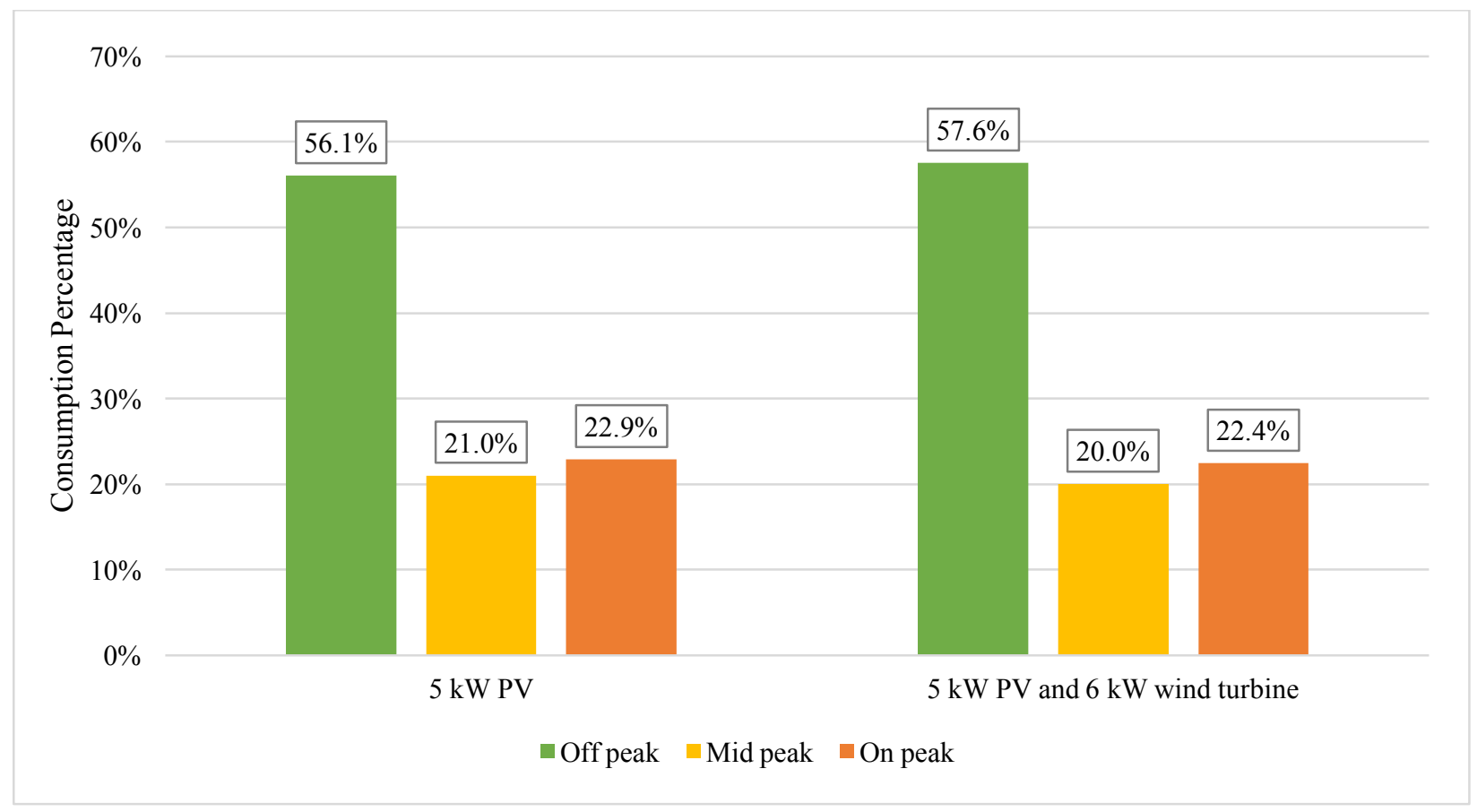

Figure 6-4: TOU consumption distribution for the medium household using zero-feedback renewable energy systems

\subsubsection{Baseline Energy Cost}

The total annual electricity costs for the entire simulated home are presented in Table 6-9. The values shown in Table 6-9 include fixed annual costs (\$346.68/year) and tax (5\%). It is clear that this represents a substantial cost for a homeowner, which much needed room for improvement.

Table 6-9: Total simulated annual electricity costs

\begin{tabular}{|l|c|c|}
\hline House type & Average annual cost & Average monthly cost \\
\hline Low consumption & $\$ 3,438.30$ & $\$ 286.53$ \\
\hline Medium consumption & $\$ 3,941.00$ & $\$ 328.42$ \\
\hline High consumption & $\$ 4,671.50$ & $\$ 389.29$ \\
\hline
\end{tabular}

Table 6-10 and Table 6-11 show the cost breakdown by energy end-use and appliance usage characteristics. These tables do not consider fixed costs or tax, since these amounts are calculated 
based on the whole billed amount. Table 6-11 in particular clearly illustrates the importance of energy conservation and efficiency when it comes to choosing appliances and how they are used.

Table 6-10: Total simulated variable electricity costs associated with space conditioning and water heating

\begin{tabular}{|l|c|}
\hline Energy end-use & Cost \\
\hline Space heating and cooling & $\$ 729.73$ \\
\hline Air handling unit & $\$ 359.00$ \\
\hline Heat recovery ventilator & $\$ 533.59$ \\
\hline Domestic hot water (electric) & $\$ 607.79$ \\
\hline Total & $\mathbf{\$ 2 , 2 3 0 . 1 1}$ \\
\hline
\end{tabular}

Table 6-11: Total simulated variable electricity costs for each of the three appliance usage characteristics.

\begin{tabular}{|l|c|}
\hline Appliance use type & Average cost \\
\hline Low consumption & $\$ 697.83(\sigma=\$ 9.34)$ \\
\hline Medium consumption & $\$ 1,176.60(\sigma=\$ 12.05)$ \\
\hline High consumption & $\$ 1,872.30(\sigma=\$ 15.93)$ \\
\hline
\end{tabular}

\subsubsection{Baseline Cost with Renewables on a Zero-Feedback Basis}

The baseline operating cost savings associated with the renewable energy systems was calculated on a zero-feedback basis. This means that the systems would never export any excess electricity generation to the grid - their instantaneous power generation is either used at the time it is generated, or the device's inverter throttles the generation. This framework was assumed because it considers the renewable energy system's minimum contribution to reducing the operating cost of a home. On a zero-feedback basis, ever-changing energy policies are ignored. Costs calculated on this basis are a safe estimate of the minimum savings potential associated with the addition of a renewable energy system.

Figure 6-5 summarizes the annual electricity costs of the three different home types with each of the considered renewable energy systems, with some combinations of systems as well. This clearly illustrates that, on its own, solar PV has the most substantial impact of reducing electricity costs for a home owner, yielding annual savings of $20.6 \%, 19.4 \%$, and $17.8 \%$ for the low, medium, and high consumption homes respectively. 


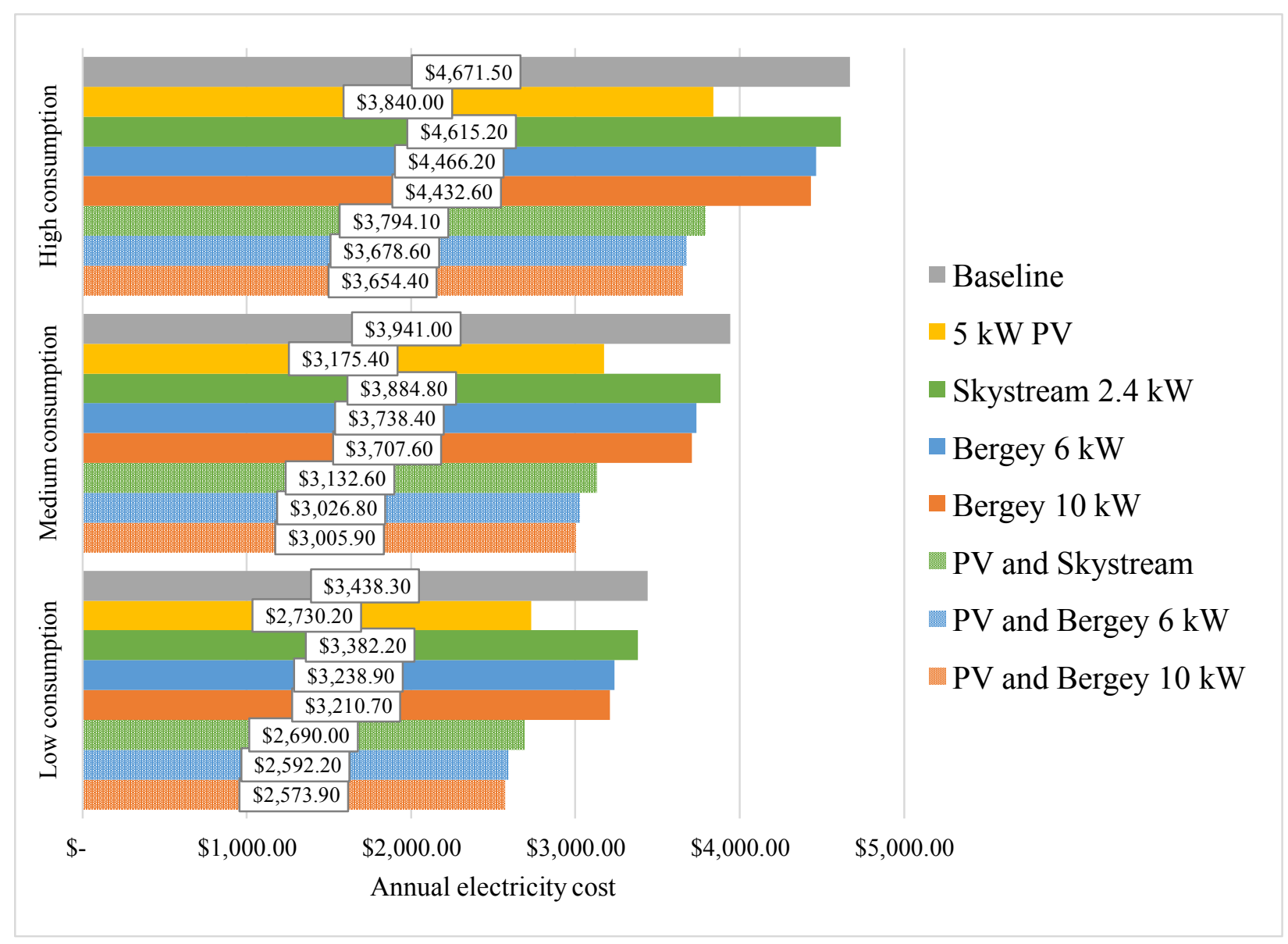

Figure 6-5: Cost comparison of zero-feedback renewable generation systems

This clearly illustrates the shortcomings of residential renewable energy systems without financial incentives or energy storage. Table 6-12 shows the capital costs of each system as installed. All capital costs were converted to Canadian dollars on a basis of $\$ 1.35 \mathrm{CAD}=\$ 1.00 \mathrm{USD}$. It is clear that at current prices, the $5 \mathrm{~kW} \mathrm{PV}$ system is the only option that would come close to paying back within a foreseeable timeline. However, with an estimated simple payback of $24-28$ years, this is far from ideal.

Clearly, additional measures are required in order to reduce the operational costs to the home owner, and facilitate greater renewable energy utilization. The following sections investigate energy storage and management techniques aimed achieving energy savings greater than the $20 \%$ achievable by a simple zero-feedback PV system. 
Table 6-12: Renewable energy system capital costs as installed

\begin{tabular}{|l|c|}
\hline System & Capital cost (installed) \\
\hline Roof-mounted solar PV $(5 \mathrm{~kW})$ & $\$ 20,115$ (NREL, 2016) \\
\hline Skystream $2.4 \mathrm{~kW}$ wind turbine & $\$ 16,200-20,250$ (Southwest Windpower, Inc., 2013) \\
\hline Bergey $6 \mathrm{~kW}$ wind turbine & $\$ 29,693$ (Bergey Windpower Co., 2017) \\
\hline Bergey $10 \mathrm{~kW}$ wind turbine & $\$ 64,800-87,750$ (Bergey Windpower Co., 2017) \\
\hline
\end{tabular}

\subsection{Deterministic Energy Management Systems}

\subsubsection{Load Shifting without Renewables}

The first case of load shifting investigated involves no renewable generation - only household loads and a battery bank. This most basic form of load shifting functions solely based on the TOU schedule presented in Section 3.1.1; during mid- and on-peak hours, the battery bank outputs power equal to the consumption of the home. Between 19:00 and 7:00, the battery bank is then allowed to recharge from the grid.

The annual savings of this practice are highly dependent on both battery size and TOU prices. Assuming fixed TOU prices, load shifting saves more money annually as battery capacity is increased. The usefulness of the size increase can be found up until a point where the battery sufficiently covers all (or the majority) of the peak load for the entire year. Any increase in size after this plateau point needlessly increases the capital cost of the battery with no financial benefit to the homeowner. The determination of this optimal battery bank size is of interest, and is a function of the amount of load consumed by a home.

Figure 6-6, Figure 6-7, and Figure 6-8 illustrate this concept for the low and high consumption homes respectively. It is clear that after a certain point, any increase in battery size becomes unnecessary. While these figures illustrate the region in which this plateau occurs, Table 6-13 summarizes the optimal battery sizes, along with their annual savings for six different cases: the three whole-house electricity consumption cases, and the three different appliance loads alone. Six cases (as opposed to only the three whole-house cases) were used to show that a general trend holds true over a wide range of residential loads. Figure 6-9 showcases the relationship between battery bank size, household load, and the number of days annually that the battery bank is fully depleted using this particular control strategy. 


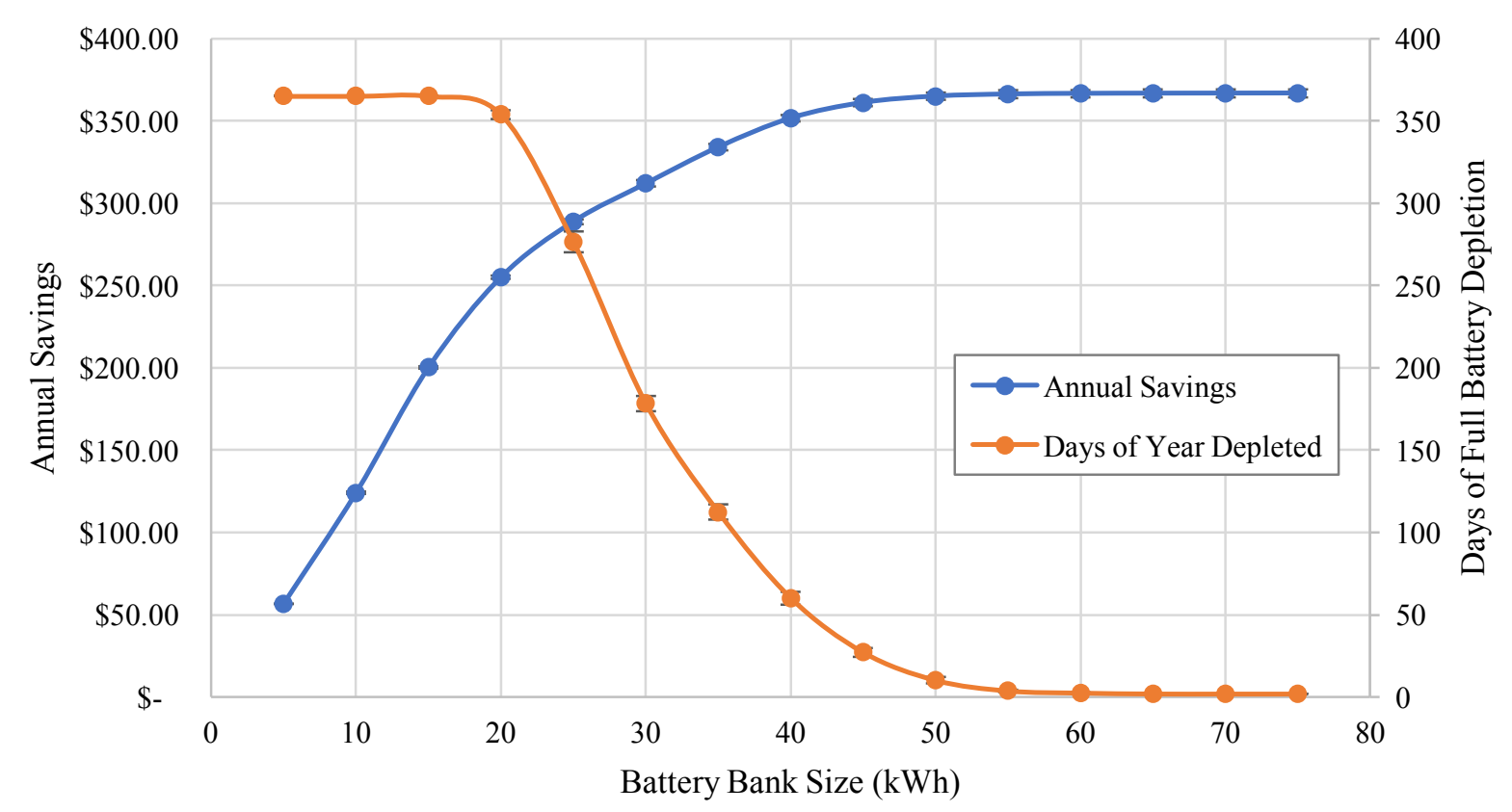

Figure 6-6: Annual load shifting cost savings for load shifting based on battery size for the lowenergy consumption house

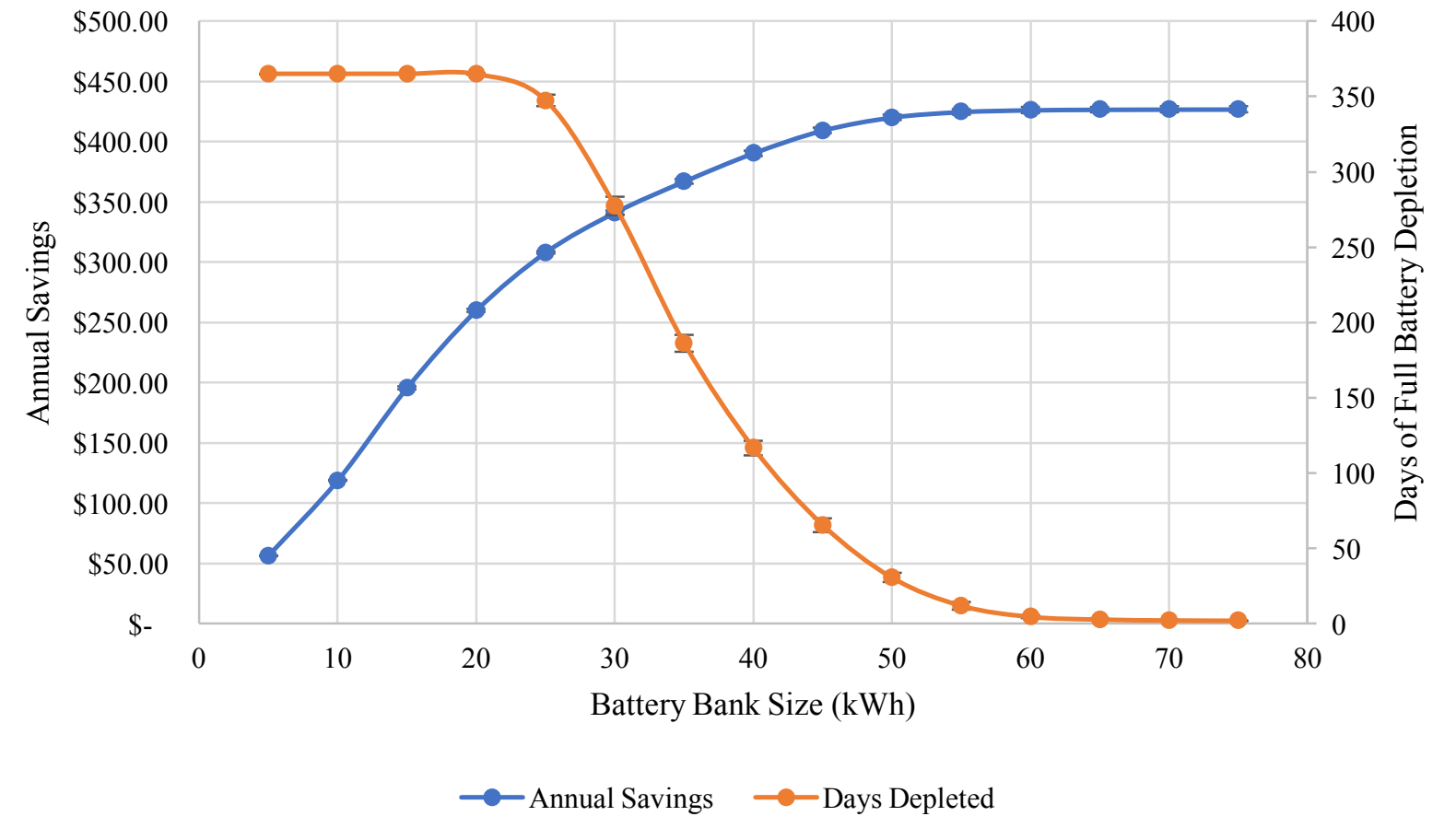

Figure 6-7: Annual load shifting cost savings for load shifting based on battery size for the medium-energy consumption house 


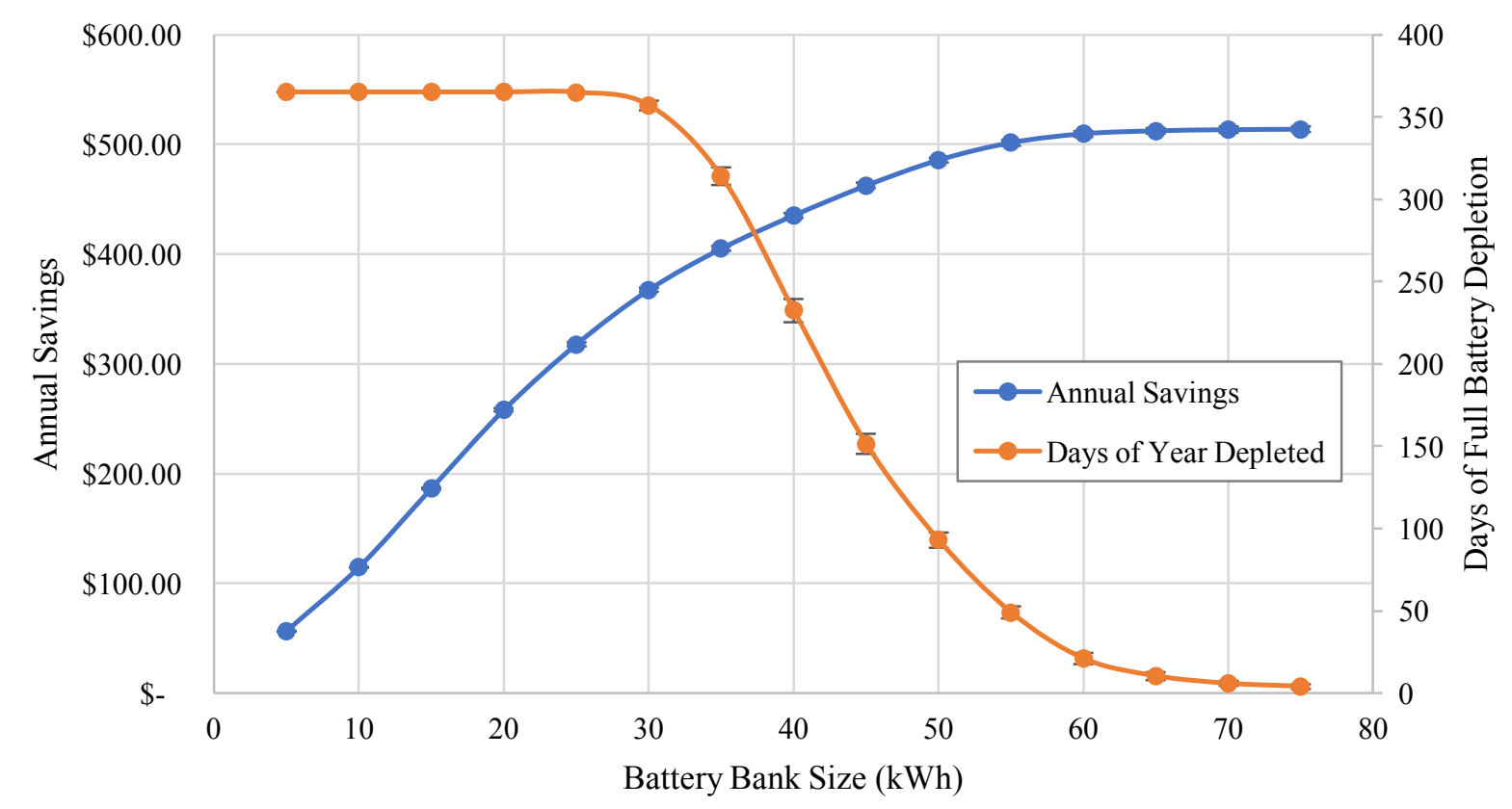

Figure 6-8: Annual load shifting cost savings for load shifting based on battery size for the highenergy consumption house

Table 6-13: Annual load shifting savings summary for different load types

\begin{tabular}{|l|c|c|c|c|c|c|}
\hline \multicolumn{1}{|c|}{ Load type } & $\begin{array}{c}\text { Annual } \\
\text { consumption } \\
(\mathrm{kWh})\end{array}$ & $\begin{array}{c}\text { Daily } \\
\text { Average } \\
(\mathrm{kWh})\end{array}$ & $\begin{array}{c}\text { Initial } \\
\text { cost }(\$)\end{array}$ & $\begin{array}{c}\text { Load } \\
\text { shifted } \\
\text { cost }(\$)\end{array}$ & $\begin{array}{c}\text { Cost } \\
\text { savings } \\
(\%)\end{array}$ & $\begin{array}{c}\text { 5-day } \\
\text { depletion } \\
\text { battery bank } \\
\text { size (kWh) }\end{array}$ \\
\hline $\begin{array}{l}\text { Low Appliance } \\
\text { Consumption }\end{array}$ & 4,815 & 13.2 & $\$ 1,096.73$ & $\$ 1,006.99$ & $8.2 \%$ & 15.3 \\
\hline $\begin{array}{l}\text { Medium } \\
\text { Appliance } \\
\text { Consumption }\end{array}$ & 8,149 & 22.3 & $\$ 1,599.41$ & $\$ 1,450.16$ & $9.3 \%$ & 22.7 \\
\hline $\begin{array}{l}\text { High Appliance } \\
\text { Consumption }\end{array}$ & 12,993 & 35.6 & $\$ 2,329.92$ & $\$ 2,093.69$ & $10.1 \%$ & 32 \\
\hline $\begin{array}{l}\text { Low Whole- } \\
\text { House } \\
\text { Consumption }\end{array}$ & 20,408 & 55.9 & $\$ 3,438.35$ & $\$ 3,072.33$ & $10.6 \%$ & 54.1 \\
\hline $\begin{array}{l}\text { Medium Whole- } \\
\text { House } \\
\text { Consumption }\end{array}$ & 23,794 & 65.2 & $\$ 3,941.02$ & $\$ 3,514.78$ & $10.8 \%$ & 59.8 \\
\hline $\begin{array}{l}\text { High Whole- } \\
\text { House } \\
\text { Consumption }\end{array}$ & 28,586 & 78.3 & $\$ 4,671.53$ & $\$ 4,157.91$ & $11.0 \%$ & 72.5 \\
\hline
\end{tabular}




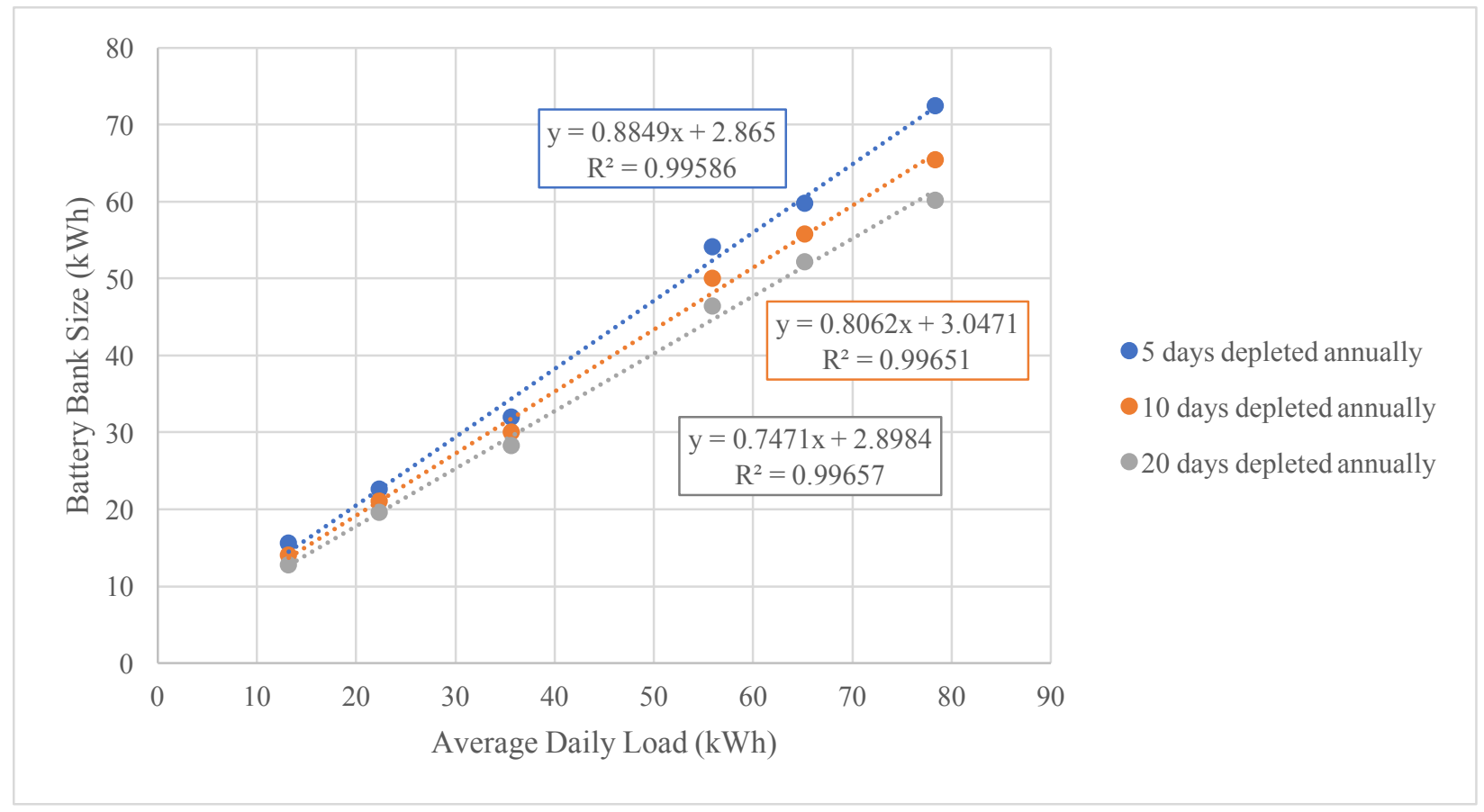

Figure 6-9: Optimal battery bank size for varying household loads based on basic load shifting control and number of days depleted annually

For this analysis, the "optimal" savings plateau was assumed to occur at the point when the battery was depleted for only five days of the year. This number was chosen arbitrarily to represent sufficiently good load coverage, and Figure 6-9 displays the resulting change in battery bank size when different numbers of annual depletion days are considered. This analysis presents a clear correlation between the electricity consumption of a home and the optimal battery size to perform basic load shifting based on the Ontario TOU schedule. The correlation could be used as a sizing tool in the design phase when considering the installation of a residential battery bank.

The resulting TOU distribution after using load shifting shows over $99 \%$ of grid consumption occurring during the off-peak period. Load shifting without renewables also results in increased annual consumption from the grid, due to the battery's charging and discharging efficiency. While this practice does reduce electricity costs, it results in an average $12 \%$ increase in grid consumption, pictured in Figure 6-10. Therefore, load shifting without renewables could only be considered "green" in jurisdiction with a very low grid emission factor, or when the grid emission factor is lower at night than during the day, as it is in Ontario. 


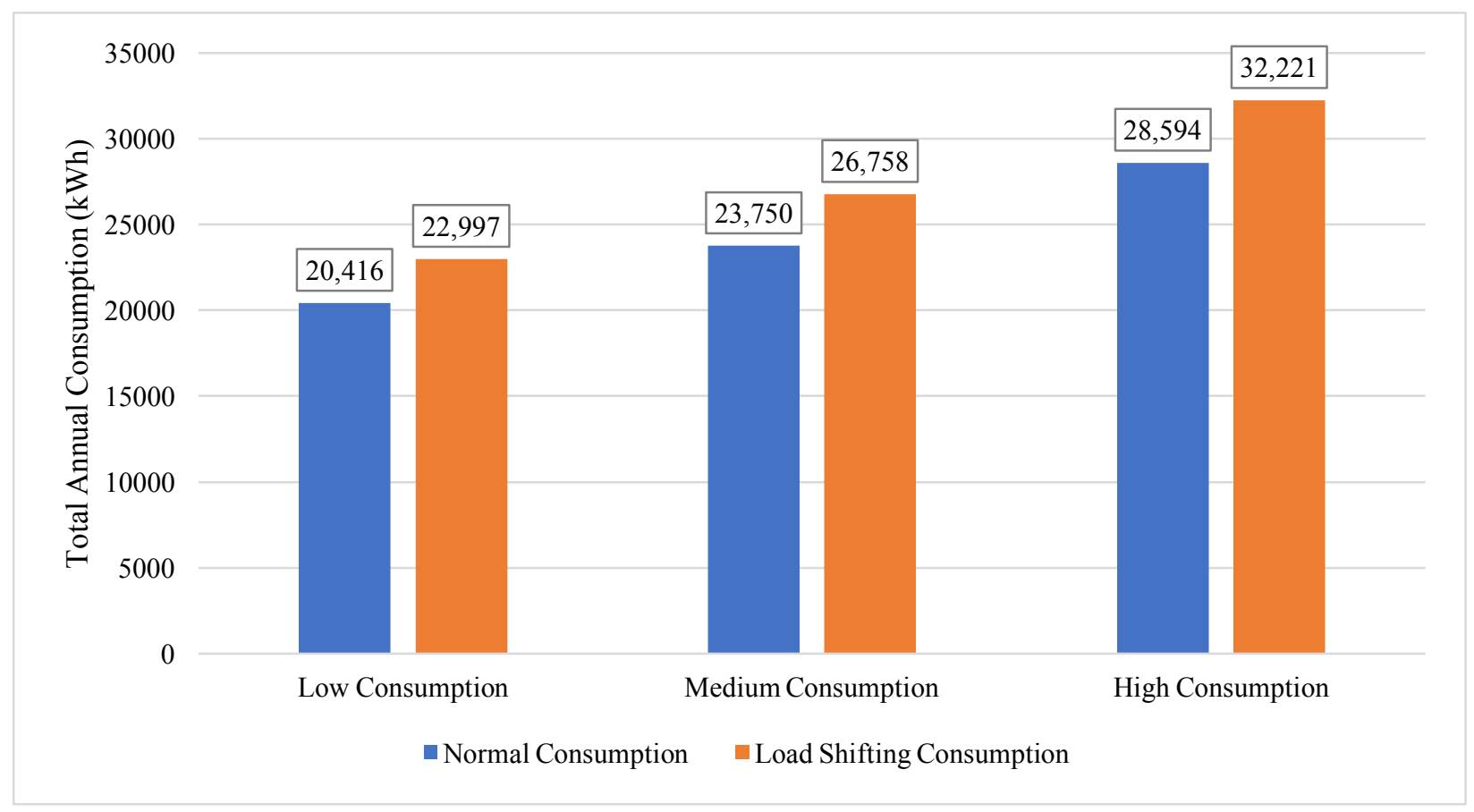

Figure 6-10: Changes in annual consumption using load shifting without renewables 


\subsubsection{Basic Load Shifting with Renewables}

The same plateau behaviour observed with basic load shifting case is observed when adding zerofeedback DGs. However, the addition of renewables shows potential reductions in optimal battery bank sizes between $4-14 \mathrm{kWh}$ depending on load and DG system size. Table 6-14 summarizes these potential savings for a system with a $5 \mathrm{~kW}$ PV system, while Table 6-15 shows potential savings with the addition of a $6 \mathrm{~kW}$ wind turbine. Note that the initial costs shown are based on zero-feedback operation without a battery bank, and cost savings relative to normal operation are even greater. When compared to normal operation (no renewables or battery storage), this configuration achieves $26-29 \%$ annual cost savings.

Table 6-14: Annual load shifting/zero-feedback savings summary for different load types and optimal battery size assuming a $5 \mathrm{~kW}$ zero-feedback $P V$ system

\begin{tabular}{|l|c|c|c|c|c|c|}
\hline \multicolumn{1}{|c|}{ Load type } & $\begin{array}{c}\text { Annual } \\
\text { consumption } \\
(\mathrm{kWh})\end{array}$ & $\begin{array}{c}\text { Daily } \\
\text { average } \\
\mathrm{kWh})\end{array}$ & $\begin{array}{c}\text { Initial } \\
\text { cost }(\$)\end{array}$ & $\begin{array}{c}\text { Load } \\
\text { shifted } \\
\text { cost }(\$)\end{array}$ & $\begin{array}{c}\text { Cost } \\
\text { savings } \\
(\%)\end{array}$ & $\begin{array}{c}\text { 5-day } \\
\text { depletion } \\
\text { battery bank } \\
\text { size (kWh) }\end{array}$ \\
\hline $\begin{array}{l}\text { Low Appliance } \\
\text { Consumption }\end{array}$ & 4,815 & 13.2 & $\$ 842.97$ & $\$ 796.56$ & $5.5 \%$ & 11.6 \\
\hline $\begin{array}{l}\text { Medium } \\
\text { Appliance } \\
\text { Consumption }\end{array}$ & 8,149 & 22.3 & $\$ 1,216.90$ & $\$ 1,133.38$ & $6.9 \%$ & 17.4 \\
\hline $\begin{array}{l}\text { High Appliance } \\
\text { Consumption }\end{array}$ & 12,993 & 35.6 & $\$ 1,797.22$ & $\$ 1,652.97$ & $8.0 \%$ & 25.8 \\
\hline $\begin{array}{l}\text { Low Whole- } \\
\text { House } \\
\text { Consumption }\end{array}$ & 20,408 & 55.9 & $\$ 2,737.80$ & $\$ 2,490.04$ & $9.0 \%$ & 49.4 \\
\hline $\begin{array}{l}\text { Medium Whole- } \\
\text { House } \\
\text { Consumption }\end{array}$ & 23,794 & 65.2 & $\$ 3,183.48$ & $\$ 2,886.29$ & $9.3 \%$ & 54.6 \\
\hline $\begin{array}{l}\text { High Whole- } \\
\text { House } \\
\text { Consumption }\end{array}$ & 28,586 & 78.3 & $\$ 3,848.51$ & $\$ 3,476.39$ & $9.7 \%$ & 63.5 \\
\hline
\end{tabular}


Table 6-15: Annual load shifting/zero-feedback savings summary for different load types and optimal battery size assuming $5 \mathrm{~kW} P V$ and $6 \mathrm{~kW}$ wind turbine zero-feedback systems

\begin{tabular}{|l|c|c|c|c|c|c|}
\hline \multicolumn{1}{|c|}{ Load type } & $\begin{array}{c}\text { Annual } \\
\text { consumption } \\
(\mathrm{kWh})\end{array}$ & $\begin{array}{c}\text { Daily } \\
\text { average } \\
(\mathrm{kWh})\end{array}$ & $\begin{array}{c}\text { Initial } \\
\text { cost }\end{array}$ & $\begin{array}{c}\text { Load } \\
\text { shifted } \\
\text { cost }\end{array}$ & $\begin{array}{c}5 \text { days } \\
\text { Cost } \\
\text { savings }\end{array}$ & $\begin{array}{c}\text { depleted } \\
\text { battery bank } \\
\text { size (kWh) }\end{array}$ \\
\hline $\begin{array}{l}\text { Low Appliance } \\
\text { Consumption }\end{array}$ & 4,815 & 13.2 & $\$ 808.70$ & $\$ 751.51$ & $7.1 \%$ & 10.0 \\
\hline $\begin{array}{l}\text { Medium } \\
\text { Appliance } \\
\text { Consumption }\end{array}$ & 8,149 & 22.3 & $\$ 1,163.08$ & $\$ 1,067.75$ & $8.2 \%$ & 16.0 \\
\hline $\begin{array}{l}\text { High Appliance } \\
\text { Consumption }\end{array}$ & 12,993 & 35.6 & $\$ 1,720.10$ & $\$ 1,564.77$ & $9.0 \%$ & 24.0 \\
\hline $\begin{array}{l}\text { Low Whole- } \\
\text { House } \\
\text { Consumption }\end{array}$ & 20,408 & 55.9 & $\$ 2,592.20$ & $\$ 2,371.26$ & $8.5 \%$ & 47.5 \\
\hline $\begin{array}{l}\text { Medium Whole- } \\
\begin{array}{l}\text { House } \\
\text { Consumption }\end{array}\end{array}$ & 23,794 & 65.2 & $\$ 3,026.80$ & $\$ 2,758.84$ & $10.1 \%$ & 53.4 \\
\hline $\begin{array}{l}\text { High Whole- } \\
\text { House } \\
\text { Consumption }\end{array}$ & 28,586 & 78.3 & $\$ 3,678.60$ & $\$ 3,338.79$ & $10.3 \%$ & 60.6 \\
\hline
\end{tabular}

Figure 6-11 shows the sensitivity of 5-day depletion battery bank size to the size of the renewable energy system used. This shows that doubling the PV array, from 5 to $10 \mathrm{~kW}$, can reduce the battery size by an average of $2.6 \mathrm{kWh}$.

Figure 6-12 illustrates the correlation between load amount and optimal battery size for load shifting with a $5 \mathrm{~kW}$ PV zero-feedback system with and without a $6 \mathrm{~kW}$ small-scale wind turbine. The addition of the $6 \mathrm{~kW}$ wind turbine reduces the optimal battery bank size by an average of 1.8 $\mathrm{kWh}$. 


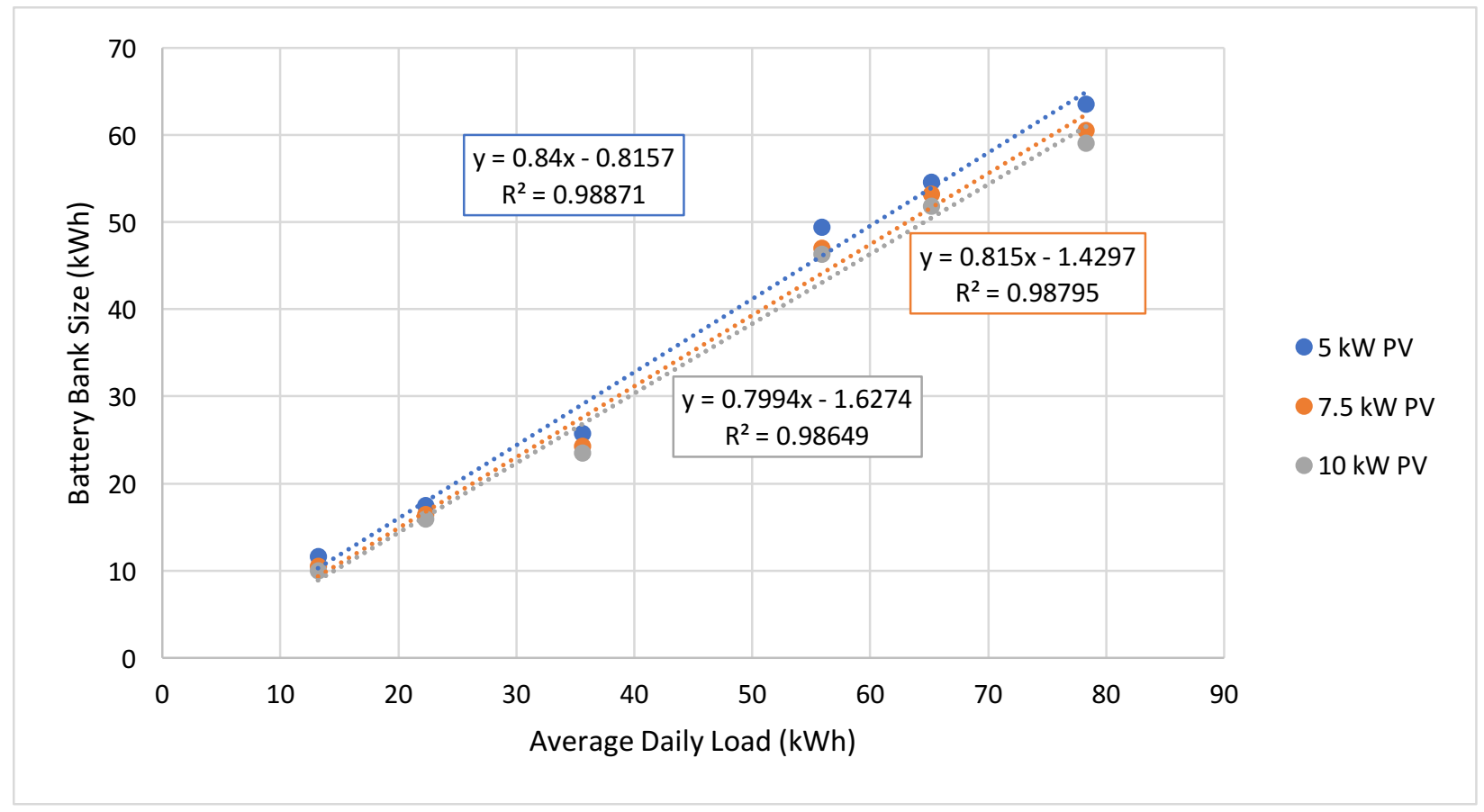

Figure 6-11: Sensitivity of battery bank size using basic load shifting to PV array size based on 5 days of annual depletion

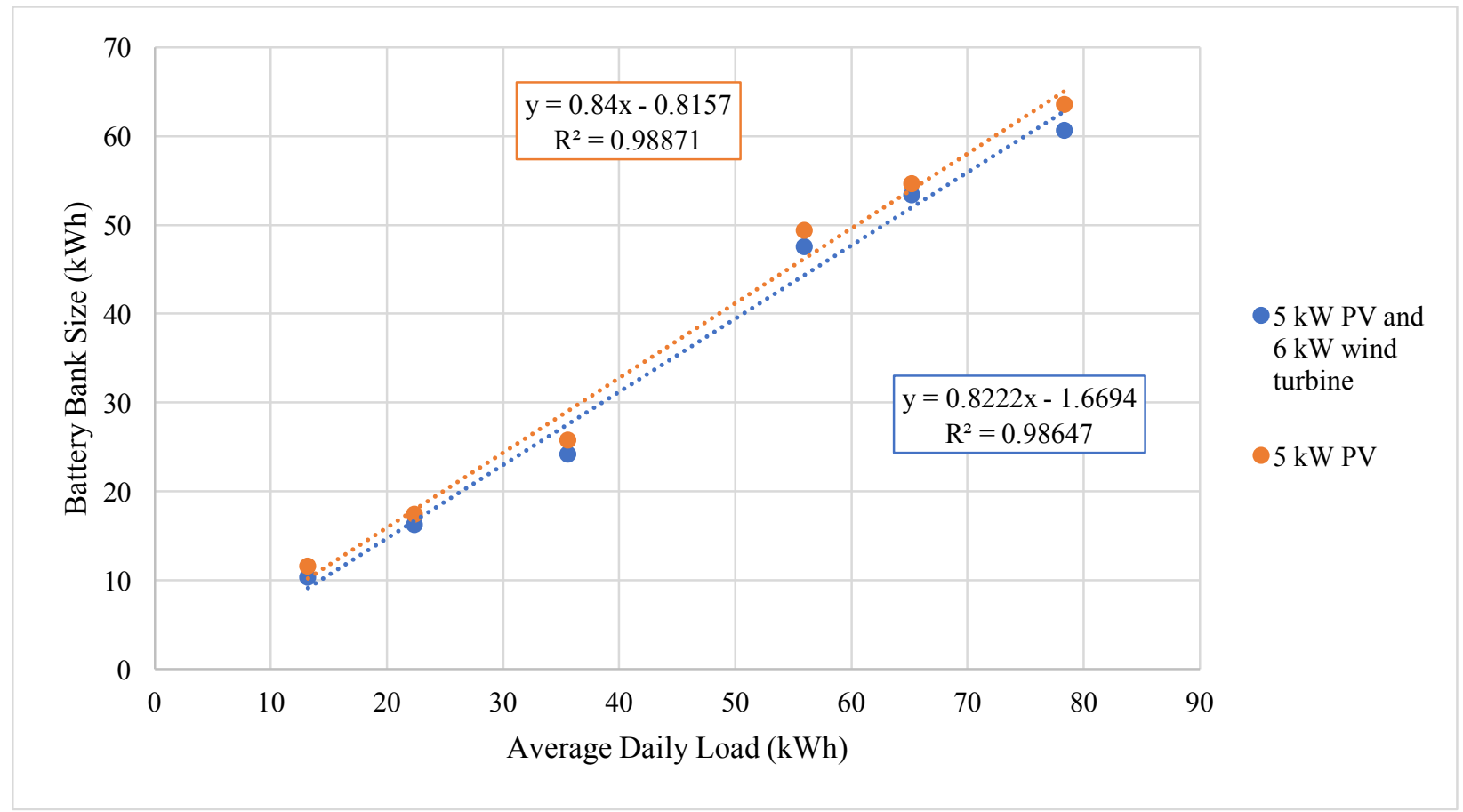

Figure 6-12:Battery bank size with and without a wind turbine based on 5 days of depletion annually using basic load shifting 


\subsubsection{Load Shifting with Excess Charging}

Table 6-16 and Table 6-17 summarize the annual savings for each of the six cases when using load shifting with excess energy storage using a $5 \mathrm{~kW}$ PV system alone and with a $6 \mathrm{~kW}$ wind turbine, respectively. Again, the saving percentages shown are relative to the cost of running the standalone renewable energy systems under a zero-feedback configuration without a battery. This control method achieves additional savings of $4-10 \%$ when compared to basic load shifting. Relative to normal operating costs without renewables, load shifting with excess charging achieves $29-36 \%$ annual cost savings. Using the excess charging method increases the amount of on-site renewable energy utilization; the only case in which excess on-site generation cannot not be used is when the battery bank is fully charged.

Table 6-16: Annual load shifting/excess energy storage savings summary for different load types and optimal battery size assuming a $5 \mathrm{~kW} P V$ system

\begin{tabular}{|l|c|c|c|c|c|c|}
\hline \multicolumn{1}{|c|}{ Load type } & $\begin{array}{c}\text { Annual } \\
\text { consumption } \\
(\mathrm{kWh})\end{array}$ & $\begin{array}{c}\text { Daily } \\
\text { Average } \\
\mathrm{kWh})\end{array}$ & $\begin{array}{c}\text { Initial } \\
\text { cost }\end{array}$ & $\begin{array}{c}\text { Load } \\
\text { shifted } \\
\text { cost }\end{array}$ & $\begin{array}{c}5 \text { days } \\
\text { Cost } \\
\text { savings }\end{array}$ & $\begin{array}{c}\text { depleted } \\
\text { battery bank } \\
\text { size (kWh) }\end{array}$ \\
\hline $\begin{array}{l}\text { Low Appliance } \\
\text { Consumption }\end{array}$ & 4,815 & 13.2 & $\$ 842.97$ & $\$ 729.50$ & $13.5 \%$ & 10.0 \\
\hline $\begin{array}{l}\text { Medium } \\
\text { Appliance } \\
\text { Consumption }\end{array}$ & 8,149 & 22.3 & $\$ 1,216.90$ & $\$ 1,023.94$ & $15.9 \%$ & 16.5 \\
\hline $\begin{array}{l}\text { High Appliance } \\
\text { Consumption }\end{array}$ & 12,993 & 35.6 & $\$ 1,797.22$ & $\$ 1,499.39$ & $16.8 \%$ & 24.9 \\
\hline $\begin{array}{l}\text { Low Whole- } \\
\text { House } \\
\text { Consumption }\end{array}$ & 20,408 & 55.9 & $\$ 2,737.80$ & $\$ 2,341.84$ & $14.5 \%$ & 49.3 \\
\hline $\begin{array}{l}\text { Medium Whole- } \\
\text { House } \\
\text { Consumption }\end{array}$ & 23,794 & 65.2 & $\$ 3,183.48$ & $\$ 2,732.89$ & $14.2 \%$ & 54.4 \\
\hline $\begin{array}{l}\text { High Whole- } \\
\text { House } \\
\text { Consumption }\end{array}$ & 28,586 & 78.3 & $\$ 3,848.51$ & $\$ 3,319.74$ & $13.7 \%$ & 63.4 \\
\hline
\end{tabular}


Table 6-17: Annual load shifting with excess charging savings summary for different load types and optimal battery size assuming $5 \mathrm{kWPV}$ and $6 \mathrm{~kW}$ wind turbine systems

\begin{tabular}{|l|c|c|c|c|c|c|}
\hline \multicolumn{1}{|c|}{ Load type } & $\begin{array}{c}\text { Annual } \\
\text { consumption } \\
(\mathrm{kWh})\end{array}$ & $\begin{array}{c}\text { Daily } \\
\text { average } \\
(\mathrm{kWh})\end{array}$ & $\begin{array}{c}\text { Initial } \\
\text { cost }\end{array}$ & $\begin{array}{c}\text { Load } \\
\text { shifted } \\
\text { cost }\end{array}$ & $\begin{array}{c}5 \text { days } \\
\text { Cost } \\
\text { savings }\end{array}$ & $\begin{array}{c}\text { depleted } \\
\text { battery bank } \\
\text { size (kWh) }\end{array}$ \\
\hline $\begin{array}{l}\text { Low Appliance } \\
\text { Consumption }\end{array}$ & 4,815 & 13.2 & $\$ 808.70$ & $\$ 684.11$ & $15.4 \%$ & 8.5 \\
\hline $\begin{array}{l}\text { Medium } \\
\text { Appliance } \\
\text { Consumption }\end{array}$ & 8,149 & 22.3 & $\$ 1,163.08$ & $\$ 952.42$ & $18.1 \%$ & 14.5 \\
\hline $\begin{array}{l}\text { High Appliance } \\
\text { Consumption }\end{array}$ & 12,993 & 35.6 & $\$ 1,720.10$ & $\$ 1,394.52$ & $18.9 \%$ & 22.5 \\
\hline $\begin{array}{l}\text { Low Whole- } \\
\text { House } \\
\text { Consumption }\end{array}$ & 20,408 & 55.9 & $\$ 2,592.20$ & $\$ 2,191.66$ & $15.5 \%$ & 47.0 \\
\hline $\begin{array}{l}\text { Medium Whole- } \\
\text { House } \\
\text { Consumption }\end{array}$ & 23,794 & 65.2 & $\$ 3,026.80$ & $\$ 2,571.73$ & $15.0 \%$ & 53.0 \\
\hline $\begin{array}{l}\text { High Whole- } \\
\text { House } \\
\text { Consumption }\end{array}$ & 28,586 & 78.3 & $\$ 3,678.60$ & $\$ 3,145.05$ & $14.5 \%$ & 60.0 \\
\hline
\end{tabular}

The sensitivity of this EMS control method to DG system size is shown in Figure 6-13. While using load shifting with excess charging, doubling the PV system size results in an average battery bank reduction of $3.7 \mathrm{kWh}$, based on the 5-day depletion criterion. Figure 6-14 displays the operation of the system with and without a $6 \mathrm{~kW}$ wind turbine. 


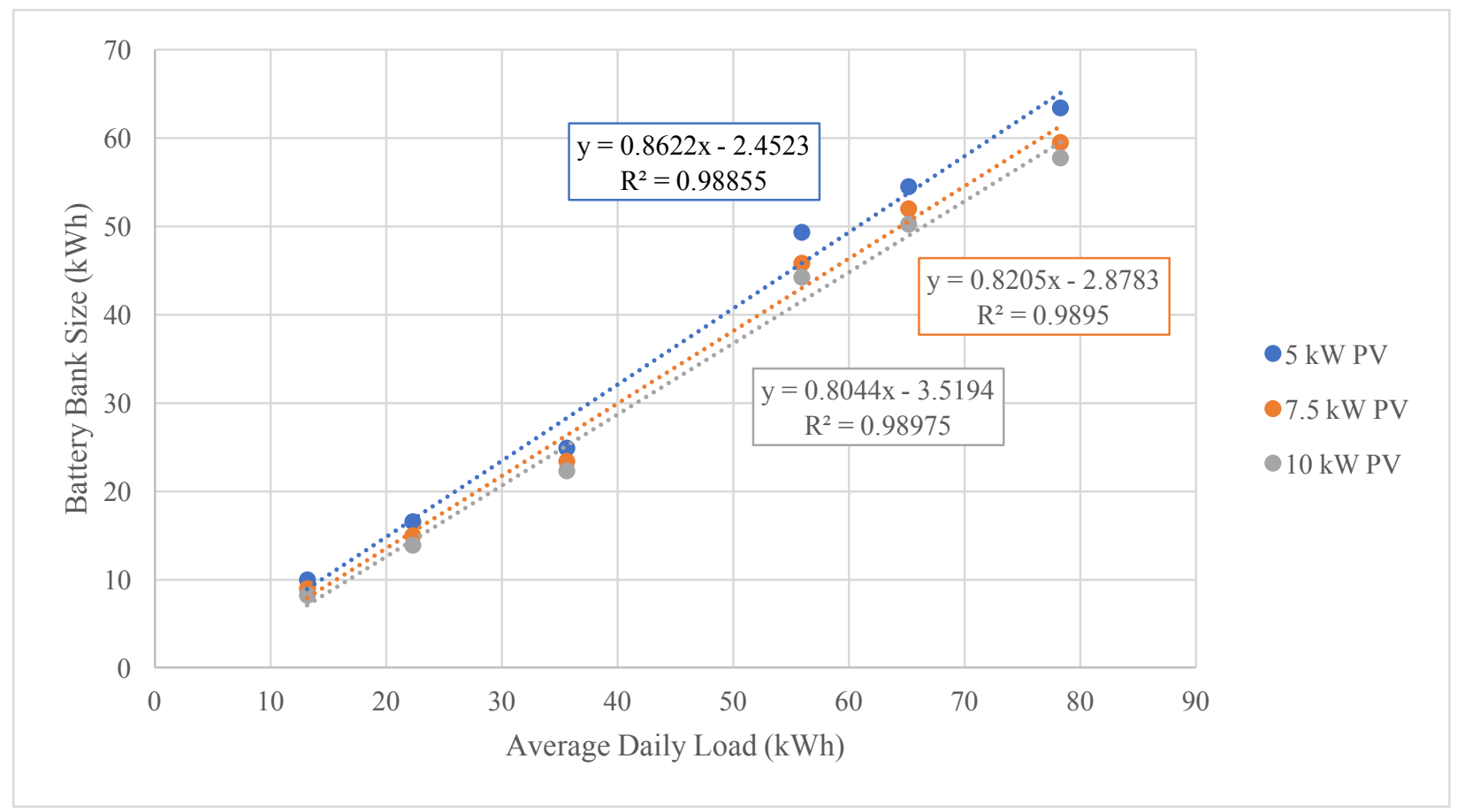

Figure 6-13: Sensitivity of battery bank size using load shifting with excess charging to PV array size based on 5 days of annual depletion

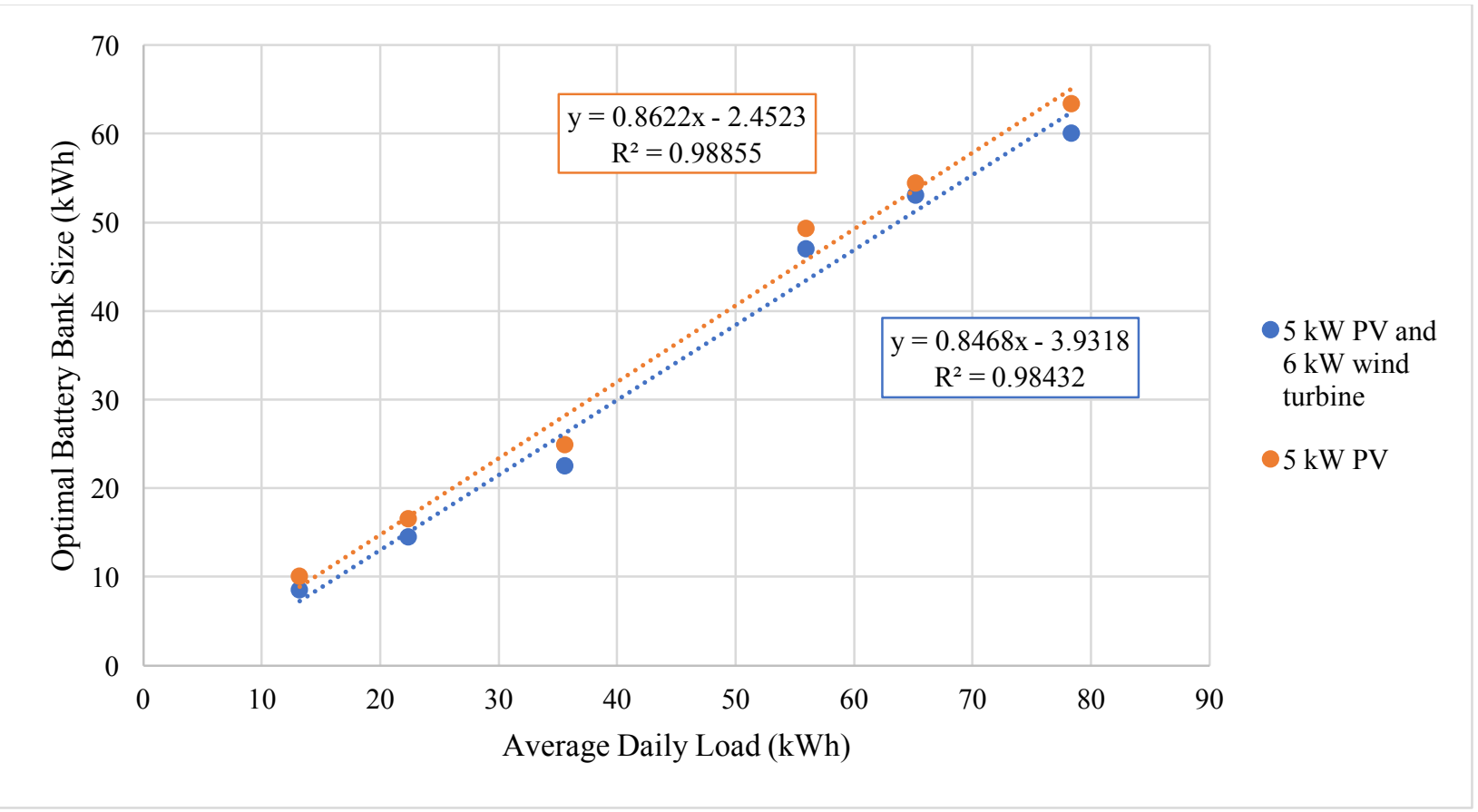

Figure 6-14: Battery bank size with and without a wind turbine based on 5 days of depletion annually using load shifting with excess charging 


\subsubsection{Deterministic Energy Management Systems Summary}

In reality, two different battery sizing curves should be produced for each of the cases highlighted. This is due to the fact that the whole-house load profiles and appliance-only load profiles have different shapes, and therefore have different TOU distributions. While the whole-house profile does have contributions from the appliance curves, it's hourly consumption is also dependent on the season, the weather, and hot water usage. This is the main reason for divergence from the trendlines. Nevertheless, very good correlations across these two types of residential load profiles are observed.

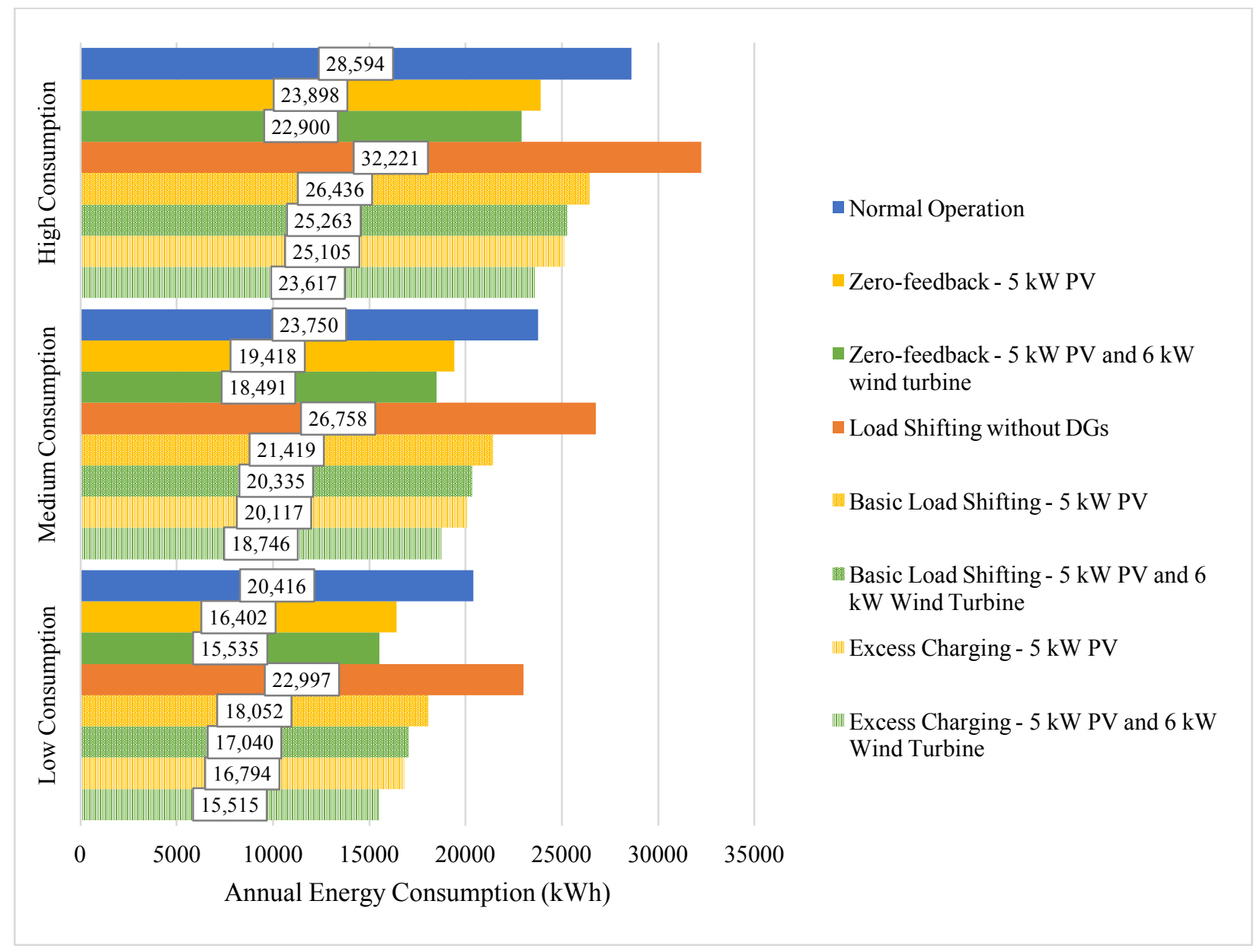

Figure 6-15: Annual energy consumption for different house types and different load shifting procedures

Figure 6-15 summarizes the annual energy consumption for all three house types under each of the different load shifting procedures investigated. Only one of the cases shows a lower energy consumption than the lowest zero-feedback case due to battery efficiency losses. 
These load shifting cases are the only EMSs investigated in this work that are appropriate for leadacid batteries; they allow a long discharging periods during the day, and long charging periods overnight. All other cases are assumed to require lithium ion batteries for real-world operation.

\subsection{Optimized Energy Management System}

The optimized battery management case gives insight into the best times of day to enable charging and discharging, based on their definition in this work. It should be noted that according to the control protocol, if discharging is enabled and there is excess renewable generation, the battery will be charged using the available energy. Table 6-18 and Figure 6-16 show the optimal distribution of the two battery states determined by the GA.

Table 6-18: Distribution of battery state selection for the three different house types

\begin{tabular}{|l|c|c|}
\hline \multicolumn{1}{|c|}{ House Type } & $\begin{array}{c}\text { Time in discharging- } \\
\text { enabled state }\end{array}$ & $\begin{array}{c}\text { Time in charging- } \\
\text { enabled state }\end{array}$ \\
\hline Low consumption & $88.8 \%$ & $11.2 \%$ \\
\hline Medium consumption & $85.9 \%$ & $14.1 \%$ \\
\hline High consumption & $81.7 \%$ & $18.3 \%$ \\
\hline
\end{tabular}

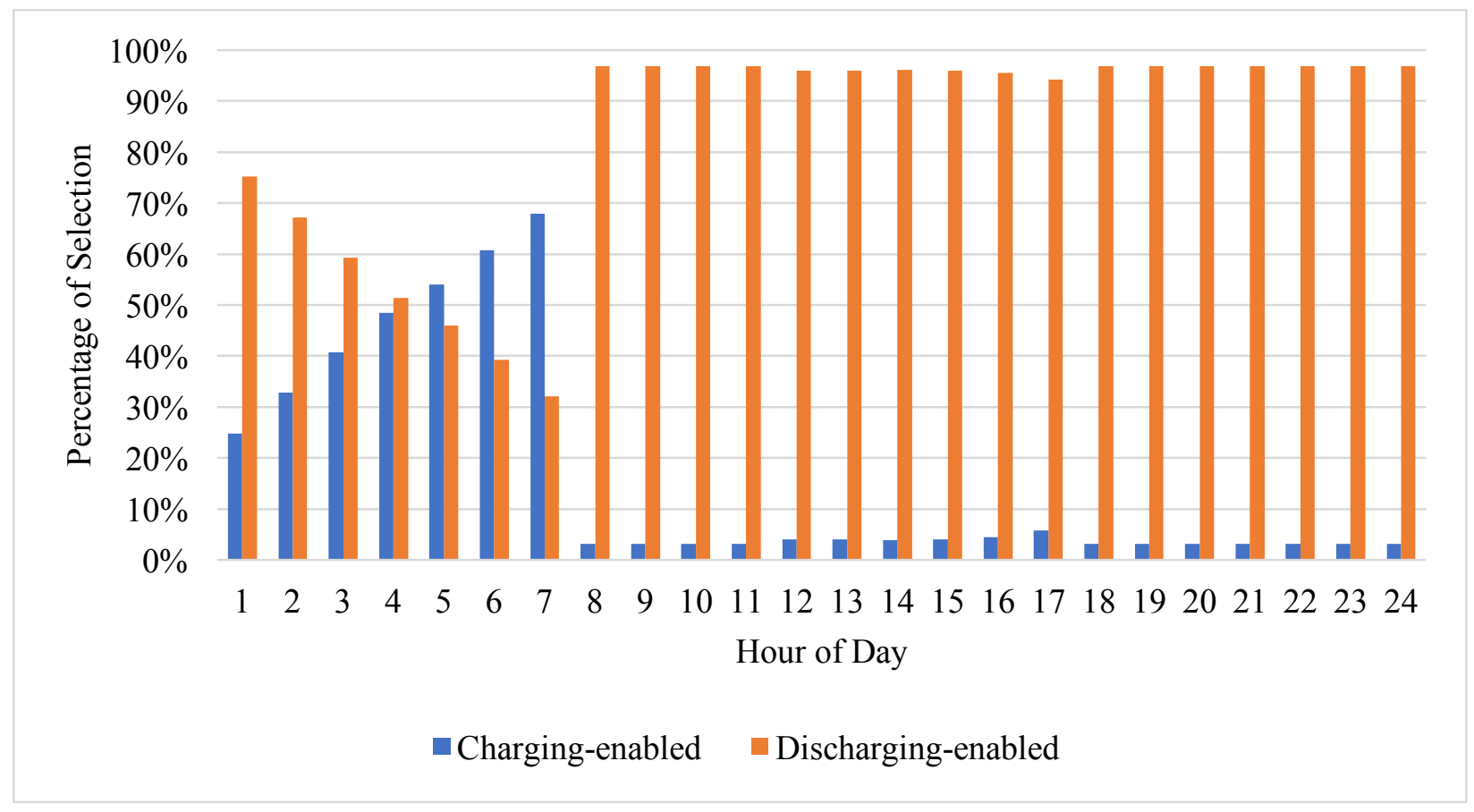

Figure 6-16: Hourly distribution of battery state selection for the medium-consumption house 
Figure 6-17 compares the annual energy consumption associated with normal operation, load shifting with excess charging, and optimized battery management, while Table 6-19 summarizes the optimized costs for the three house types relative to zero-feedback costs. The optimized battery management protocol has minimized both the energy consumption - by maximizing on-site renewable utilization - and the annual cost - by using the cheapest energy available.

It is interesting to note that the consumption from load shifting with excess charging approaches that of the optimized case as the load of the house increases. Since the higher consumption homes already utilize more on-site renewable generation - simply due to the reduced probability of generation exceeding the load - there is less room for optimization.

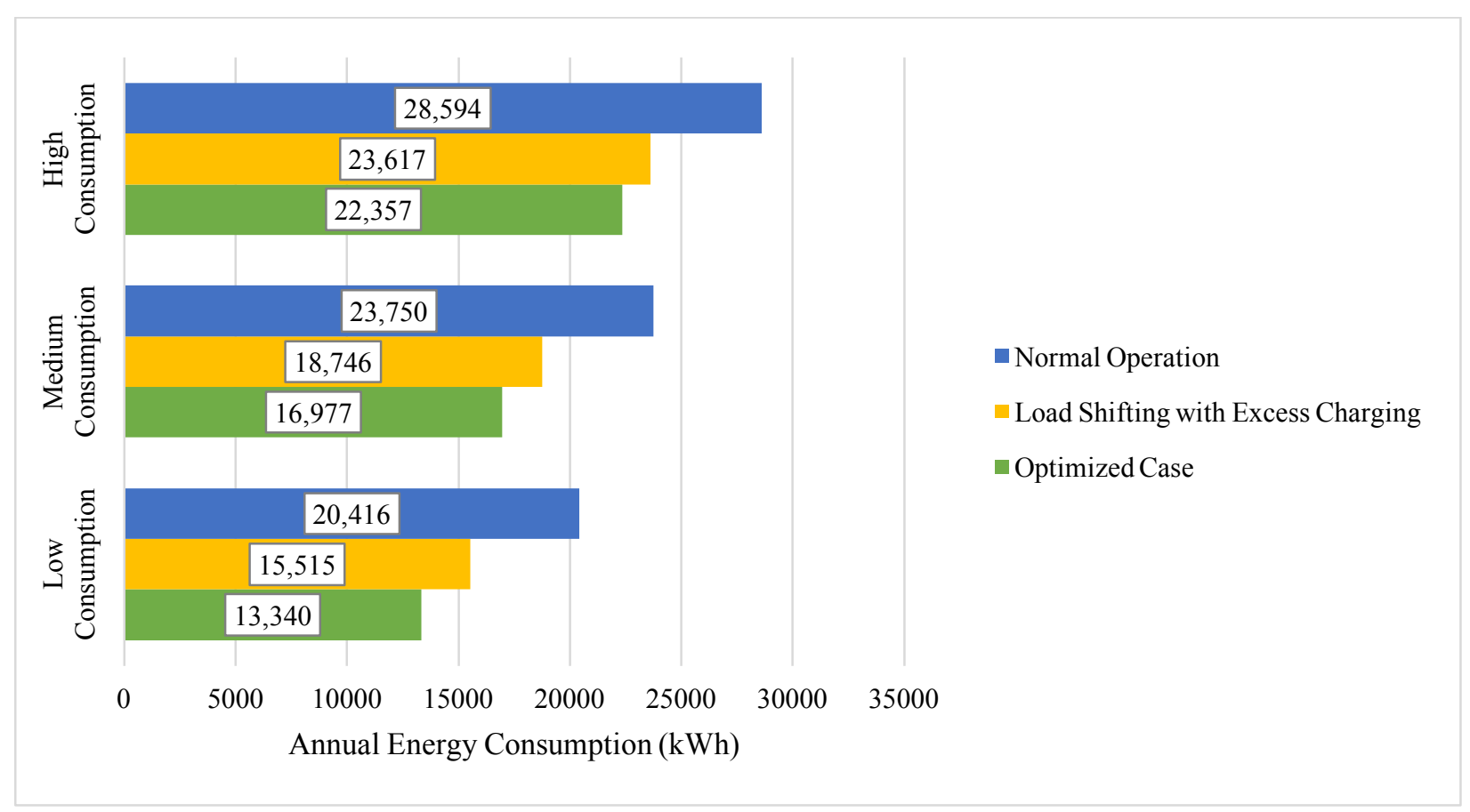

Figure 6-17: Annual energy consumption comparison between normal operation, load shifting, and optimized battery management

The optimized battery management protocol shows significant cost savings compared to zerofeedback operation. Compared to normal operation without renewables, this case shows $35-43 \%$ annual cost savings. The battery sizes used for the low, medium, and high energy consumption houses were 47, 53, and $60 \mathrm{kWh}$ - the optimal sizes listed in Table 6-17. 
Table 6-19: Cost comparison of zero-feedback operation to optimized battery management using $5 \mathrm{kWPV}$ and $6 \mathrm{~kW}$ wind turbine systems

\begin{tabular}{|l|c|c|c|c|c|}
\hline \multicolumn{1}{|c|}{ Load type } & $\begin{array}{c}\text { Annual } \\
\text { consumption } \\
(\mathrm{kWh})\end{array}$ & $\begin{array}{c}\text { Daily } \\
\text { average } \\
(\mathrm{kWh})\end{array}$ & $\begin{array}{c}\text { Zero- } \\
\text { feedback } \\
\text { cost }(\$)\end{array}$ & $\begin{array}{c}\text { Optimized } \\
\text { Cost }(\$)\end{array}$ & $\begin{array}{c}\text { Cost savings } \\
(\%)\end{array}$ \\
\hline $\begin{array}{l}\text { Low Whole- } \\
\text { House } \\
\text { Consumption }\end{array}$ & 20,408 & 55.9 & $\$ 2,592.20$ & $\$ 1,954.46$ & $24.6 \%$ \\
\hline $\begin{array}{l}\text { Medium Whole- } \\
\text { House } \\
\text { Consumption }\end{array}$ & 23,794 & 65.2 & $\$ 3,026.80$ & $\$ 2,384.93$ & $21.2 \%$ \\
\hline $\begin{array}{l}\text { High Whole- } \\
\text { House } \\
\text { Consumption }\end{array}$ & 28,586 & 78.3 & $\$ 3,678.60$ & $\$ 3,050.55$ & $17.1 \%$ \\
\hline
\end{tabular}




\subsection{Adaptive Energy Management System Results}

\subsubsection{Machine Learning Battery Management Results}

Various methods of MLS training were tested to find the optimal method of training. Figure 6-18 compares the annual cost savings for different MLS's based on the amount of data used for training; for example, the series labeled "One week training" represents an MLS that was trained using one week's worth of data, and then run on an entire year. Monthly reset training reset the memory of the MLS every month, such that it has only been trained with the previous month's data, while monthly cumulative training retrains the MLS every month, using cumulative data for the year. All of the cases shown in Figure 6-18 utilize $5 \mathrm{~kW}$ PV and $6 \mathrm{~kW}$ wind turbine systems for a medium-consumption home.

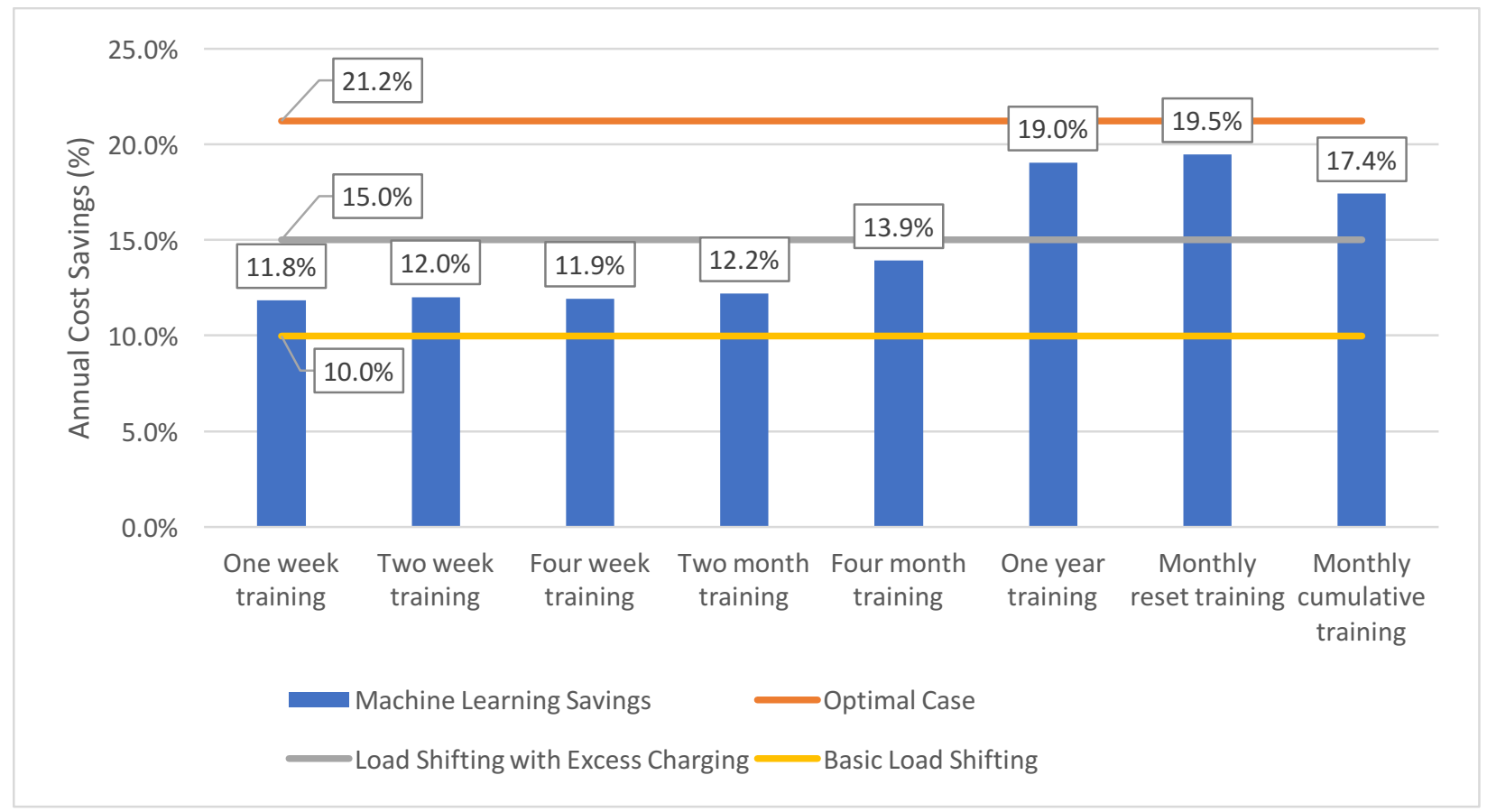

Figure 6-18: Annual cost savings associated with training the MLS with different data sets

These results show the impact of seasonal changes on the performance of the MLS. Since the monthly reset training procedure produces greater savings than cumulative training, the benefit of using more recent data to train the MLS is emphasized. In any case, these results show promise for the use of this type of MLS in a home; three of the training procedures produce greater savings 
than load shifting with excess charging, while all cases show greater savings than basic load shifting.

Table 6-20 summarizes annual energy costs associated with the three MLS's that show the best performance for each of the three house types. Since each MLS is trained using data generated through the GA optimization process, the trends in savings follow those of the optimized cases; greater savings may be achieved in cases that initially have lower amounts of on-site renewable energy utilization.

Table 6-20: Annual energy costs (and percent savings) associated with each house type using the $M L S$ with different training methods and amounts of data

\begin{tabular}{|l|c|c|c|c|}
\hline \multicolumn{1}{|c|}{ House Type } & Zero-feedback & $\begin{array}{c}\text { Training with 1 } \\
\text { year of data }\end{array}$ & $\begin{array}{c}\text { Monthly } \\
\text { cumulative } \\
\text { training }\end{array}$ & $\begin{array}{c}\text { Monthly reset } \\
\text { training }\end{array}$ \\
\hline $\begin{array}{l}\text { Low Whole- } \\
\text { House }\end{array}$ & $\$ 2,592.20$ & $\begin{array}{c}\$ 2,041.32 \\
(21.3 \%)\end{array}$ & $\begin{array}{c}\$ 2,086.54 \\
(19.5 \%)\end{array}$ & $\begin{array}{c}\$ 2,027.47 \\
(21.8 \%)\end{array}$ \\
\hline $\begin{array}{l}\text { Medium Whole- } \\
\text { Couse }\end{array}$ & $\$ 3,026.80$ & $\begin{array}{c}\$ 2,481.01 \\
(18.0 \%)\end{array}$ & $\begin{array}{c}\$ 2,530.44 \\
(16.4 \%)\end{array}$ & $\begin{array}{c}\$ 2,467.75 \\
(18.5 \%)\end{array}$ \\
\hline $\begin{array}{l}\text { High Whole- } \\
\text { House }\end{array}$ & $\$ 3,678.60$ & $\begin{array}{c}\$ 3,166.37 \\
(13.9 \%)\end{array}$ & $\begin{array}{c}\$ 3,191.04 \\
(13.3 \%)\end{array}$ & $\begin{array}{c}\$ 3,130.22 \\
(14.9 \%)\end{array}$ \\
\hline
\end{tabular}

Figure 6-19 summarizes the annual energy consumption for the three MLS cases shown in Table 6-20. All MLS cases show lower energy consumption than the baseline cases, with the same trend of decreased savings for an increase in house (load) size. Some of the cases show less grid consumption than the optimized cases. This is because the optimized cases were optimized for cost, and not energy consumption. 


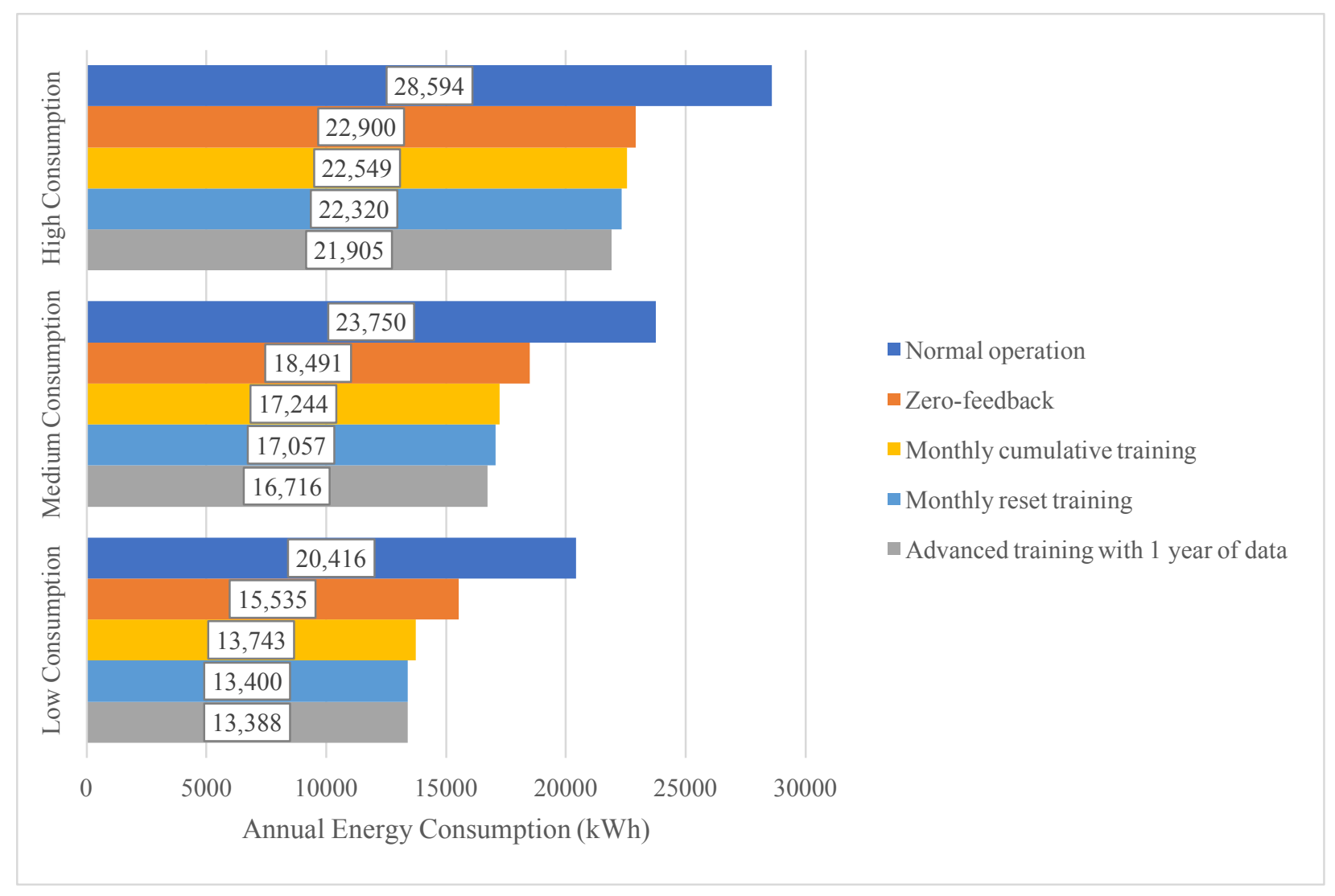

Figure 6-19: Annual energy consumption comparison between MLS's and baseline cases 


\subsubsection{Predictive Control Case Study Results}

Figure 6-20 and Figure 6-21 display the training performance of the HVAC electrical load and solar PV generation ANNs respectively, with error bars indicating the standard deviation of 30 training trials. The final HVAC and Solar PV ANNs used 9 and 10 neurons, respectively.

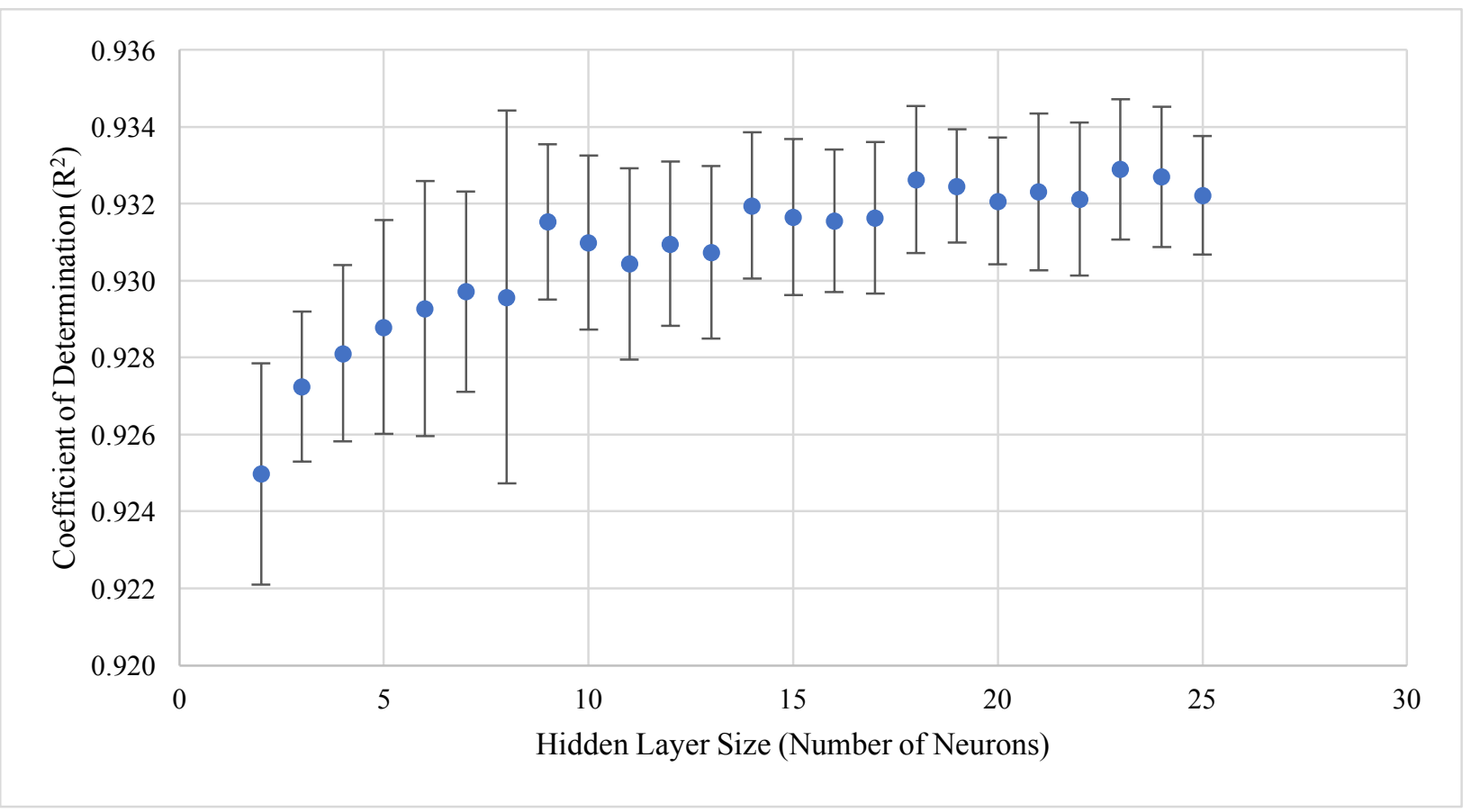

Figure 6-20: HVAC electrical load ANN based on hidden layer size for use in predictive control

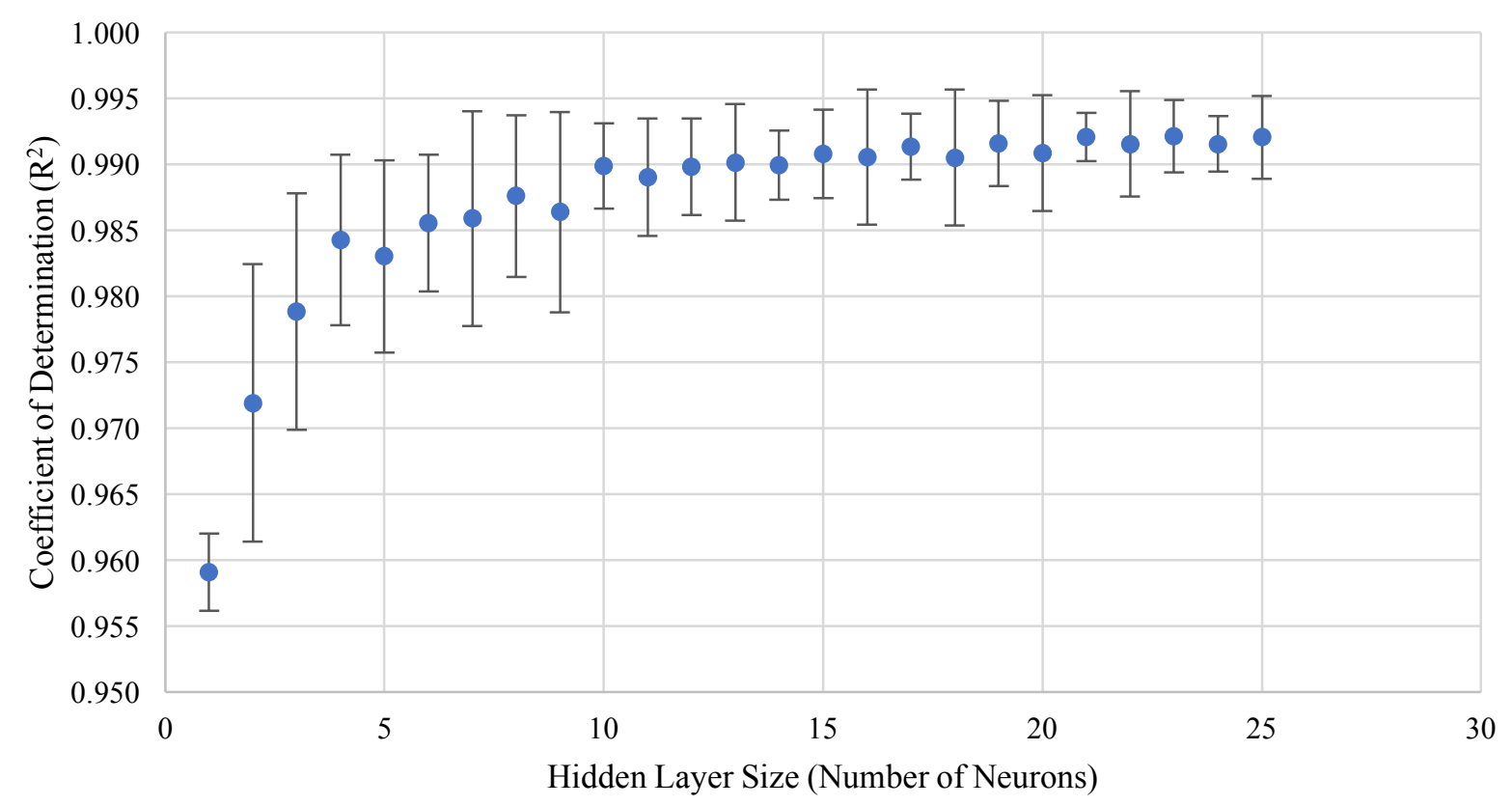

Figure 6-21: Solar PV generation ANN based on hidden layer size for use in predictive control 
The performance of both ANNs over the 10-day forecast period is shown in Figure 6-22 and. The solar PV ANN shows much better performance than the HVAC ANN, simply because a solar PV system is subject to fewer disturbances than the HVAC system of a building.

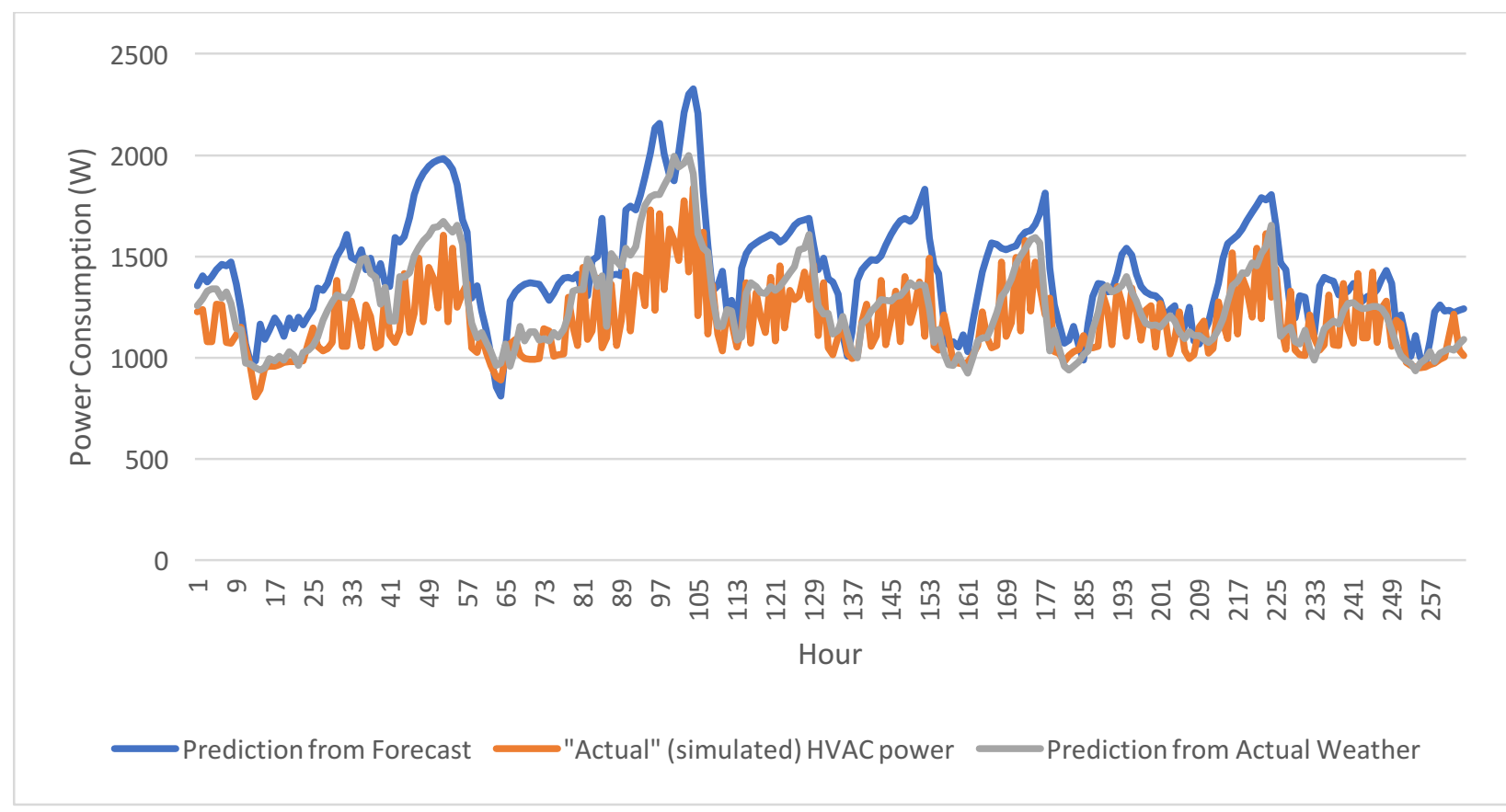

Figure 6-22: HVAC ANN output using weather forecast and actual weather data compared to "actual" (simulated) power consumption

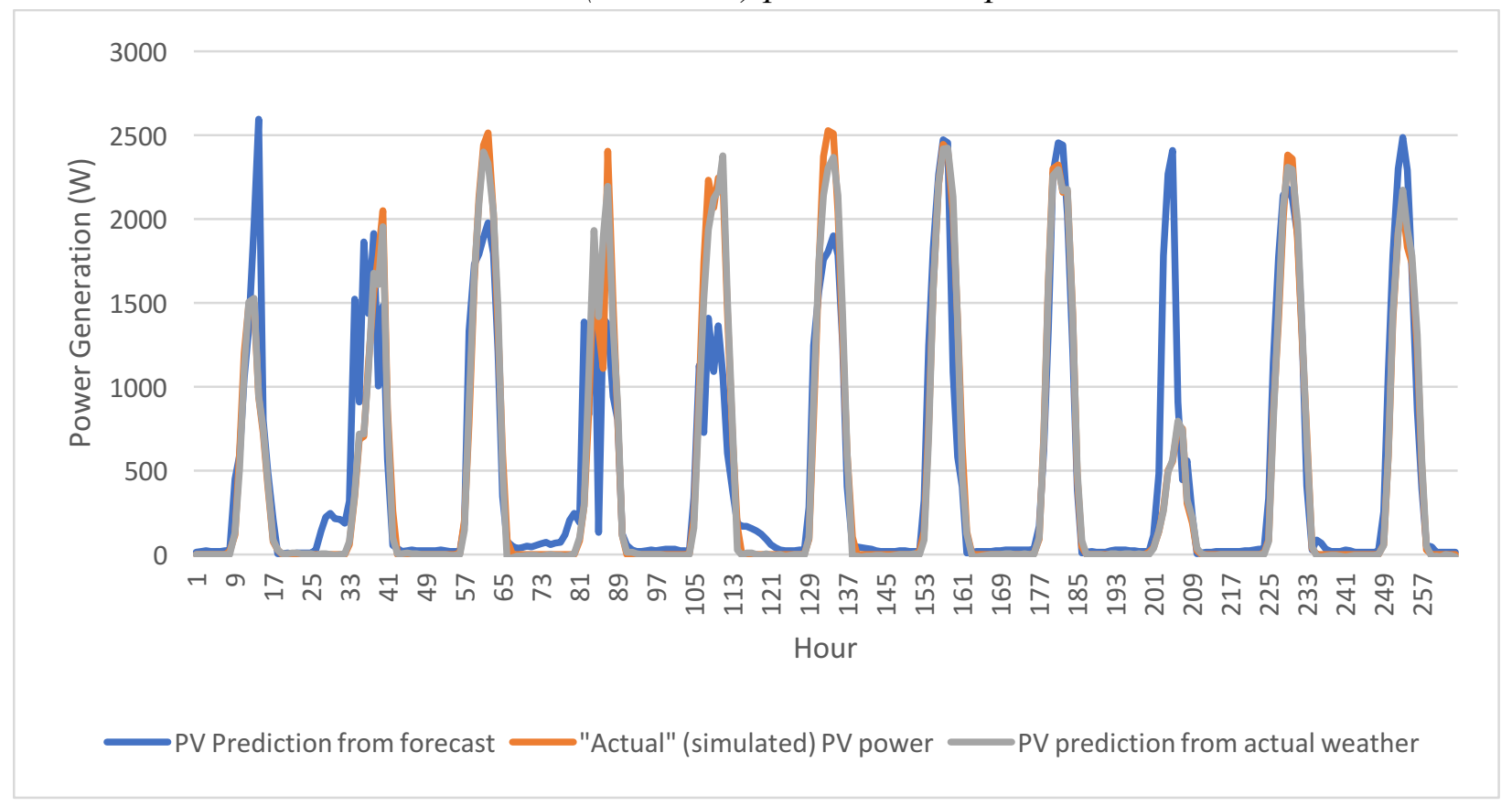

Figure 6-23: Solar PV ANN output using weather forecast and actual weather data compared to "actual" (simulated) generation 
Table 6-21 displays the $\mathrm{R}^{2}$ values for the ANN performance data summarized in Figure 6-22 and Figure 6-23. Clearly, the solar PV ANN performs much better than that of the HVAC system. The error associated with using weather forecasts as ANN inputs reflects the error associated with both the weather forecast data and the performance of the ANN models.

Table 6-21: Predictive ANN performance based on forecast or actual weather inputs

\begin{tabular}{|l|c|c|}
\hline & \multicolumn{2}{|c|}{ Correlation to simulated data $\left(\mathbf{R}^{\mathbf{2}}\right)$} \\
\hline ANN Inputs & HVAC ANN & Solar PV ANN \\
\hline Weather forecasts & 0.691 & 0.888 \\
\hline Actual weather & 0.731 & 0.995 \\
\hline
\end{tabular}

Despite the inaccuracy of the consumption and generation profiles predicted by the ANNs, predictive control has produced the greatest cost savings of any EMS investigated in this work, based on the 10-day case study period. While the annual performance of this particular form of predictive control cannot be quantified, the savings are foreseeably promising.

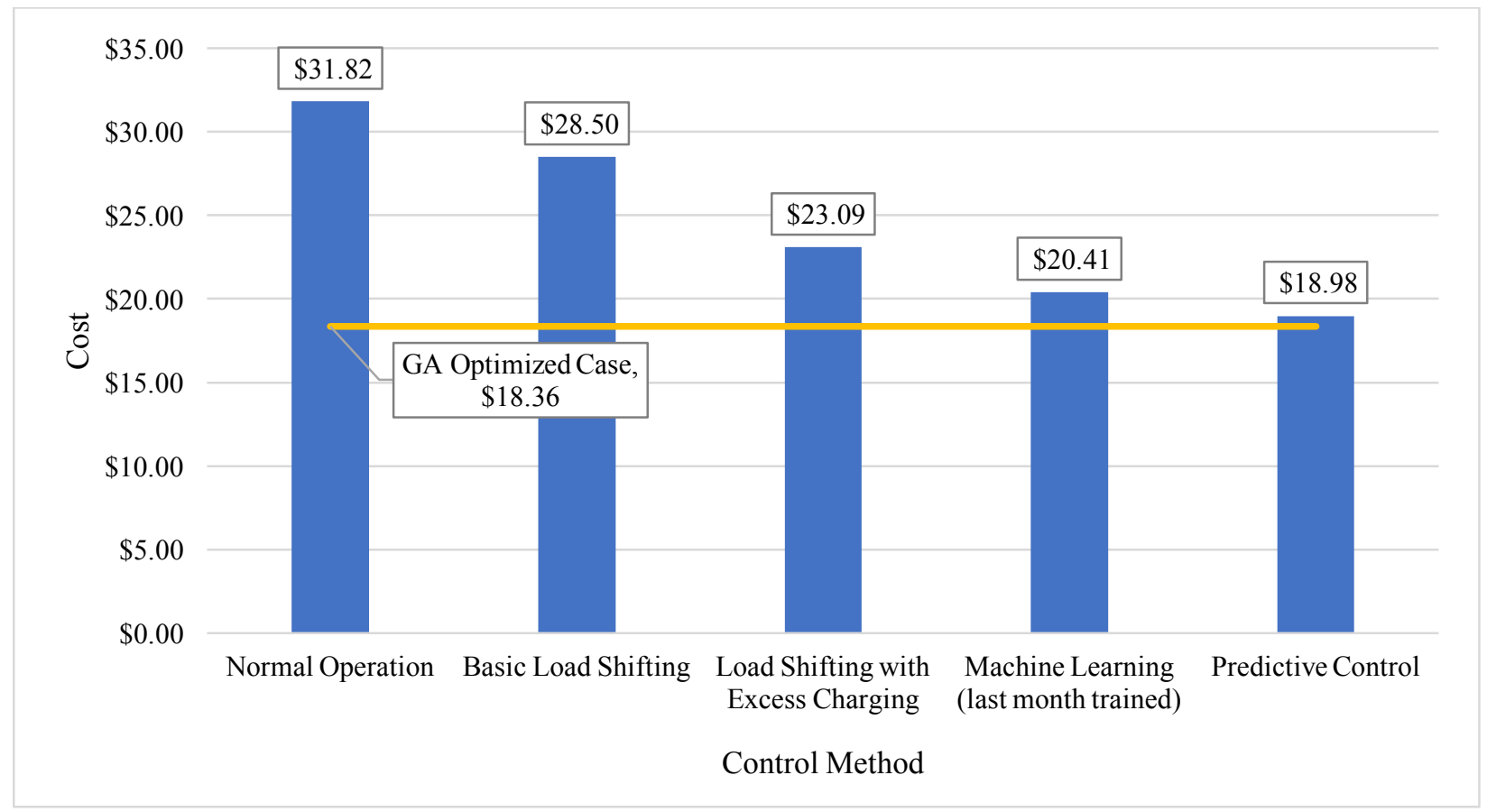

Figure 6-24: Comparison of EMS cost across the 10-day forecast period

One reason that the predictive control scheme outperforms the other EMSs is that it uses the GA optimizer to determine the best course of action for the battery bank. Because the GA optimization 
process reduces the problem of battery control to two discrete states, the problem becomes generalized. Having a generalized problem solver reduces the impact of errors associated with weather forecasts and modelling. The GA optimization process can make near optimal decisions based on general trends, and does not require exact values to produce optimal results. This method of battery management should be further investigated using annual simulations, and real-world tests.

Using an ANN to predict simulated ASHP power consumption seems to leave the results subjectable to reasonable scrutiny. However, using the ASHP ANN to model the real-world system represented by HVAC simulations gives clarity. Figure 6-25 displays the same ANN's predicted power output of the ASHP used in House A, along with the actual power consumption of the heat pump, in the real world. ASHP data was unavailable during the case study period, and this data was obtained from the first quarter of 2016.

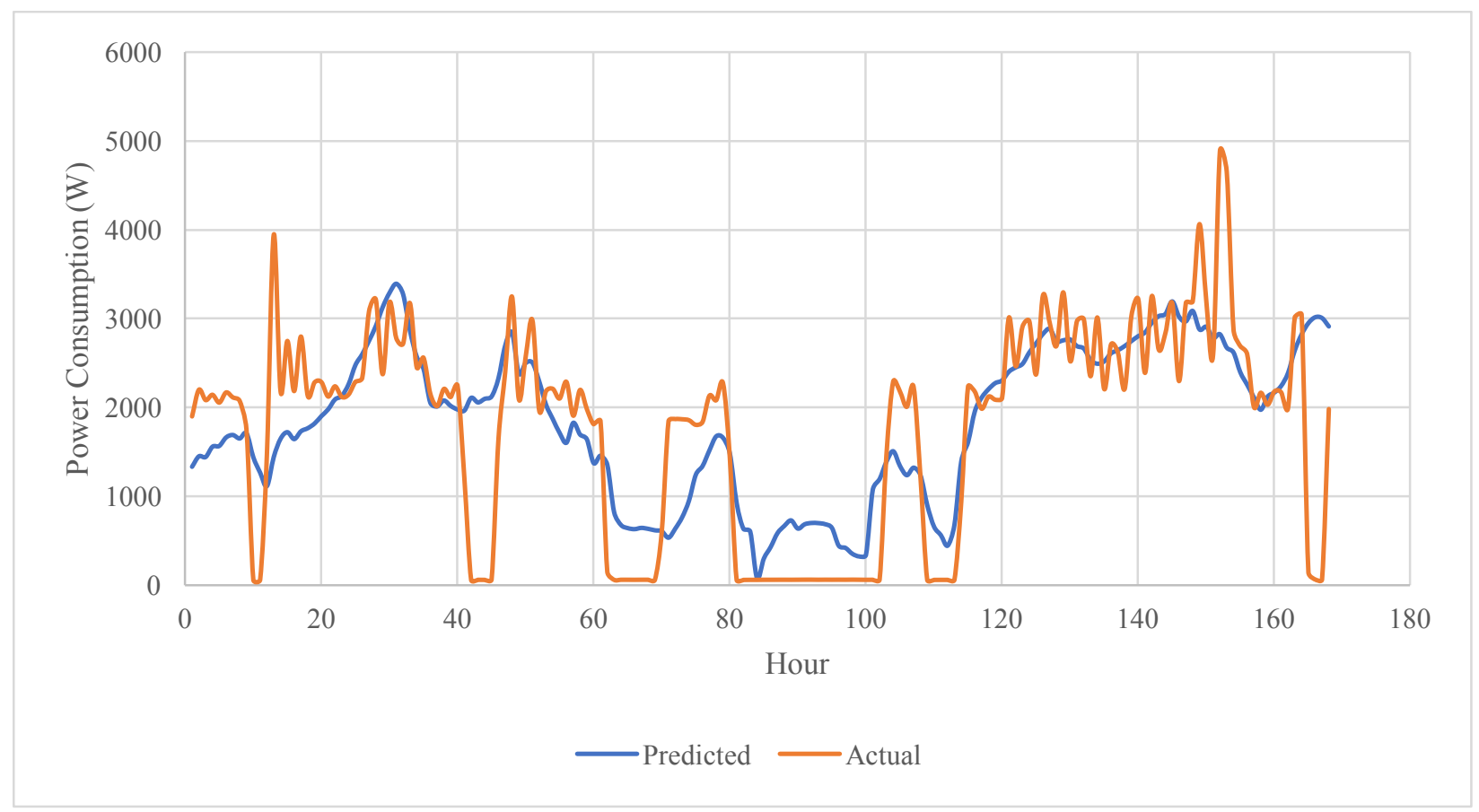

Figure 6-25: ANN-predicted versus actual ASHP Power consumption

Figure 6-25 shows some clear areas in which the ANN does not properly capture the system. However, these two results have a correlation coefficient of $\mathrm{R}^{2}=0.747$, a correlation close to that of the ANN's performance on simulated data. Since the GA optimization process has shown to 
produce near-optimal results despite poor correlations in predicted and actual data, it is highly likely that the predictive controller used in this case study would produce very similar results to those that have been reported. Experimental trials should be conducted in order to properly validate this claim.

\subsubsection{Transactive Energy Management Simulation Results}

Because the TEMS was implemented at the ASH, the systems it considered reflect exactly those which are used at the ASH. This means that the solar PV system used for all TEMS simulations was $\underline{4.08 \mathrm{~kW}}$ in size, and not $5 \mathrm{~kW}$ as in all other EMS simulations. The TEMS also only utilizes ASHPs as loads, and does not consider any other consumers of energy. When the TEMS is compared to the other EMSs investigated in this work, those EMSs have been applied to the exact same system, such that comparisons on the same basis can be made.

The TEMS is capable of producing a variety of different results. While it does not achieve as significant cost savings as some of the other EMSs investigated, it is an all-in-one EMS that is capable of many different types of control. It should be once again clarified that this work investigated only one interpretation of a transactional energy system, and that there are many other potential implementations of the framework.

A TEMS can be used as a means of DR implementation if signaling from the local utility is implemented. Figure 6-26 illustrates an example of the simulated reaction of the TEMS to a demand response signal requesting a 50\% reduction in power consumption.

The simulation pictured in Figure 6-26 used four models of House A in simultaneous operation, all with ASHPs for heating and cooling. It assumed that these houses were paired together forming two separate semi-detached buildings. Within the set, the house on the eastern side shared its west wall with the house on the western side. 


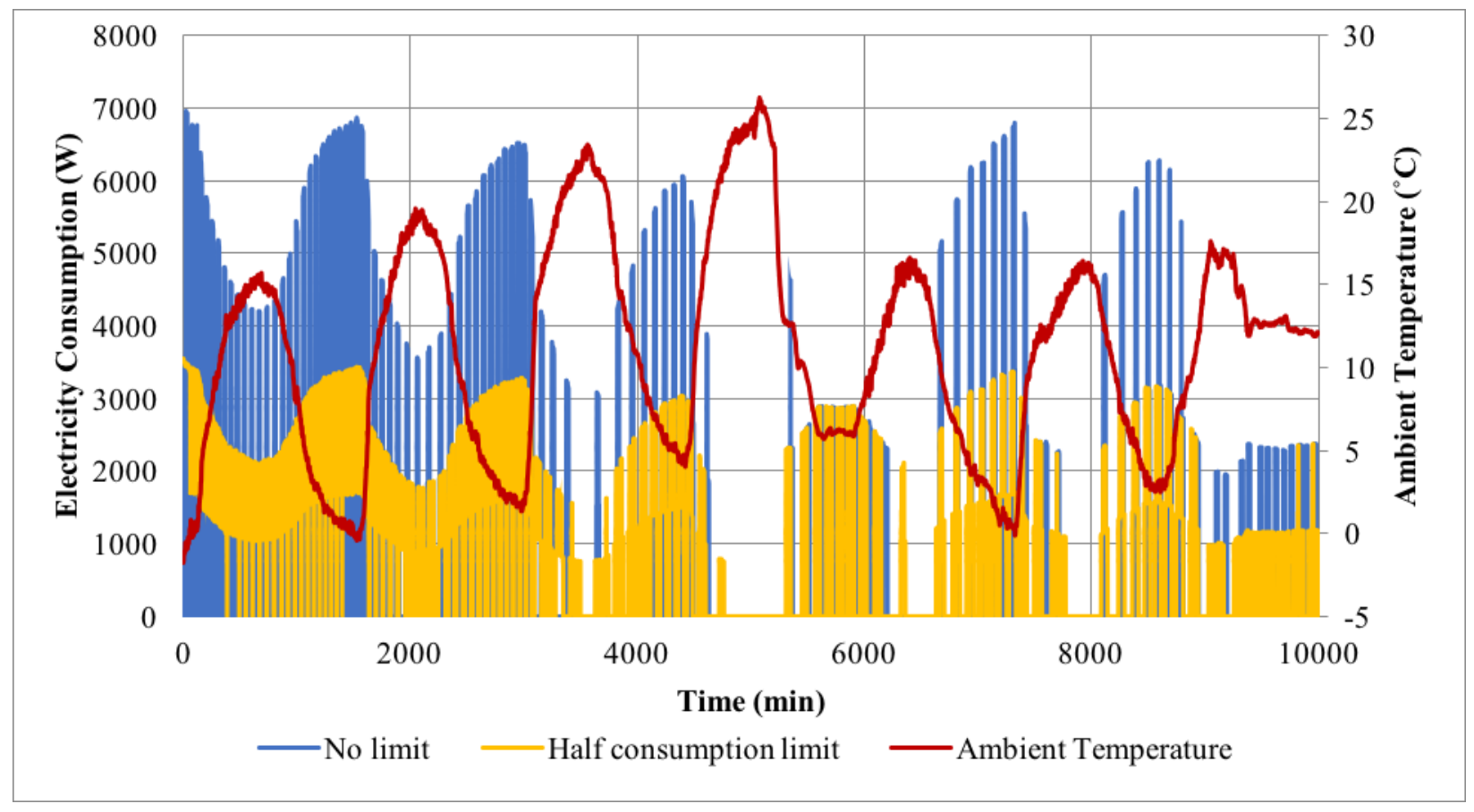

Figure 6-26: Electrical loads of four houses using a TEMS with and without a DR signal

Under the ambient conditions shown, the transactional energy system is able to manage the use of the ASHPs in the four-house community such that only two are permitted to be in operation at any given time. This is achieved by implementing a virtual demand cap on the amount of power allowed to be drawn from the grid at any point in time. This highlights the inherent ease of DR interactions between the utility and a TEMS. Implementing DR only required changing a single value in the TEMS: the grid's bid amount, in $\mathrm{kW}$, for the duration of the DR request. This requires no design changes to the TEMS whatsoever. Since it is built based on a publish-subscribe communication protocol, it is inherently built to receive and respond to this type of DR request.

While this amount of load reduction is not achievable at every point throughout the year, simulations show that this system could achieve this amount of DR for $75 \%$ of the year, from March $9^{\text {th }}-$ November $9^{\text {th }}$ based on 2016 weather data, while maintaining zone temperature between $20-25^{\circ} \mathrm{C}$. The actual demand response capability of any given system will vary depending on local weather conditions and the insulative properties of the buildings.

The TEMS can also act as a platform for implementing a dynamic temperature setback. Figure 6-27 displays four bid curves used for TEMS simulations. Each bid curve represents more relaxed 
thermal comfort preferences. These temperature setbacks and preferences can easily be applied to a TEMS, similar to modern "smart" thermostats. Table 6-22 summarizes the energy consumption and cost savings associated with each of the four bid curves shown in Figure 6-27. Actual savings would vary based on the preferences of the occupants, however, this data highlights the potential range of savings that could be achieved.

While the average temperatures shown in Table 6-22 may seem unacceptable, these are the result of a full year of operation using a fixed bid curve. In reality, bid curves would change dynamically based on season and time of day. Additional intelligence could also be added to the system to learn the habits of the occupants, dynamically modifying the bid curve of the thermostat.

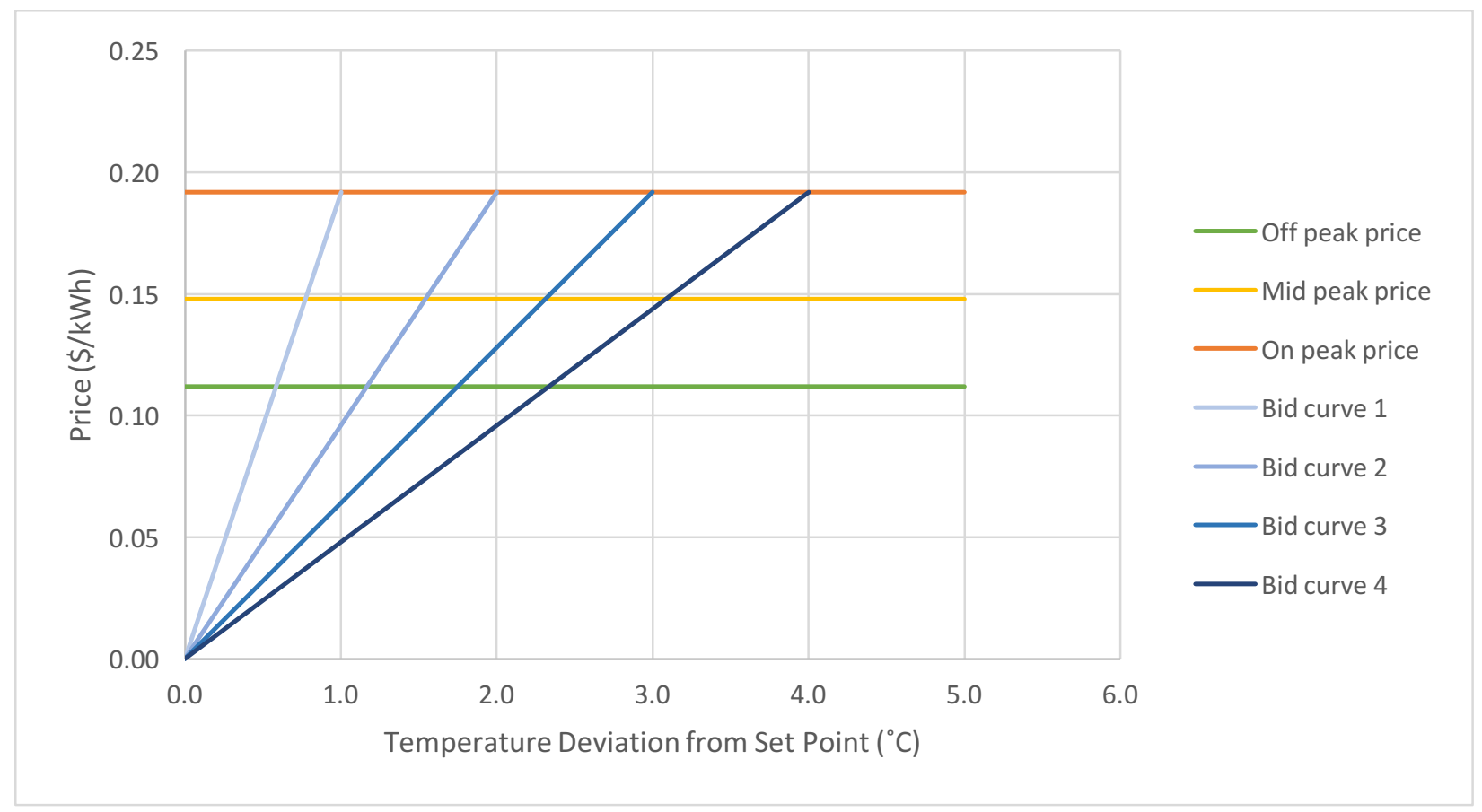

Figure 6-27: Thermostat bid curves representing different thermal comfort preferences 
Table 6-22: Summary of annual electricity savings and average zone temperatures associated with transactional energy-enabled temperature setbacks

\begin{tabular}{|l|c|c|c|c|}
\hline \multicolumn{1}{|c|}{ Trial } & $\begin{array}{c}\text { Consumption } \\
\mathbf{( k W h )}\end{array}$ & $\begin{array}{c}\text { Annual } \\
\text { variable cost } \\
\mathbf{( \$ )}\end{array}$ & $\begin{array}{c}\text { Average indoor } \\
\text { heating season } \\
\text { temperature }\left({ }^{\circ} \mathbf{C}\right)\end{array}$ & $\begin{array}{c}\text { Average indoor } \\
\text { cooling season } \\
\text { temperature }\left({ }^{\circ} \mathbf{C}\right)\end{array}$ \\
\hline $\begin{array}{l}\text { Normal ASHP } \\
\text { operation }\end{array}$ & 5183 & $\$ 729.73$ & 22.0 & 23.8 \\
\hline Bid curve 1 & 5021 & $\$ 700.28$ & 21.8 & 23.8 \\
\hline Bid curve 2 & 4676 & $\$ 645.03$ & 21.1 & 24.4 \\
\hline Bid curve 3 & 4353 & $\$ 593.74$ & 20.4 & 24.9 \\
\hline Bid curve 4 & 4057 & $\$ 547.62$ & 19.7 & 25.4 \\
\hline
\end{tabular}

The addition of renewable generation and battery energy storage in a transactive energy network has the potential to produce electricity cost savings in certain scenarios. Table 6-23 compares the calculated savings of the TEMS to normal operation. For the $4.08 \mathrm{~kW}$ solar PV system, normal operation is considered to use a zero-feedback system.

Table 6-23: Energy cost and consumption comparison of the TEMS to normal operation

\begin{tabular}{|l|c|c|c|c|}
\hline \multicolumn{1}{|c|}{ System } & $\begin{array}{c}\text { Normal } \\
\text { annual } \\
\text { consumption } \\
\mathbf{( k W h )}\end{array}$ & $\begin{array}{c}\text { Transactional } \\
\text { annual } \\
\text { consumption } \\
\mathbf{( k W h )}\end{array}$ & $\begin{array}{c}\text { Normal annual } \\
\text { variable cost }\end{array}$ & $\begin{array}{c}\text { Transactional } \\
\text { annual variable } \\
\text { cost }\end{array}$ \\
\hline ASHP & 5183 & 5021 & $\$ 729.73$ & $\$ 700.28$ \\
\hline $\begin{array}{l}\text { ASHP, } \\
\text { Solar PV }(4.08 \mathrm{~kW})\end{array}$ & 4362 & 4187 & $\$ 592.88$ & $\$ 563.15$ \\
\hline $\begin{array}{l}\text { ASHP, } \\
\text { Solar PV }(4.08 \\
\mathrm{kW}), \\
\text { Battery }(25 \mathrm{kWh})\end{array}$ & $4367-4749$ & 4253 & $\$ 477.25-535.47$ & $\$ 489.42$ \\
\hline
\end{tabular}

The upper and lower ranges associated with the battery bank system represent basic load shifting and load shifting with excess charging, respectively. It should be noted that the battery bank 
bidding procedure utilizes an unoptimized fuzzy logic controller to determine what price to bid, and whether to bid towards the supply or demand curve. There is very likely a battery bidding structure for the TEMS that will produce greater cost savings.

While the resulting electricity cost of the TEMS is $2.6 \%$ greater than that of load shifting with excess charging employed on the same system, the grid consumption associated with the TEMS is $2.6 \%$ lower than that of load shifting.

The TEMS is not explicitly designed to optimize costs, and therefore the absolute lowest costs of all investigated systems should not be expected. Nevertheless, the inherent flexibility associated with the bidding structure of the ASHPs allows the TEMS to align loads with generation when practical, leading to reduced consumption from the grid, and greater on-site renewable energy utilization. Figure 6-28 showcases an example consumption-generation alignment made possible by the TEMS. By utilizing a flexible bidding structure, a TEMS can wait a certain amount of time before granting the bid of an ASHP, increasing the probability that it can be supplemented by onsite renewable energy generation. This phenomenon could be further improved through the use of predictive control, to allow for preheating or precooling if a deficit of renewable energy generation was forecast.

On-site renewable energy utilization with and without the use of a TEMS is summarized in Table 6-24. Clearly the battery bank adds a significant amount of flexibility to the system, doubling the amount of on-site renewable energy usage. The system consisting of the ASHP and PV also has a certain amount of flexibility that allows for a $20 \%$ increase in on-site utilization. 


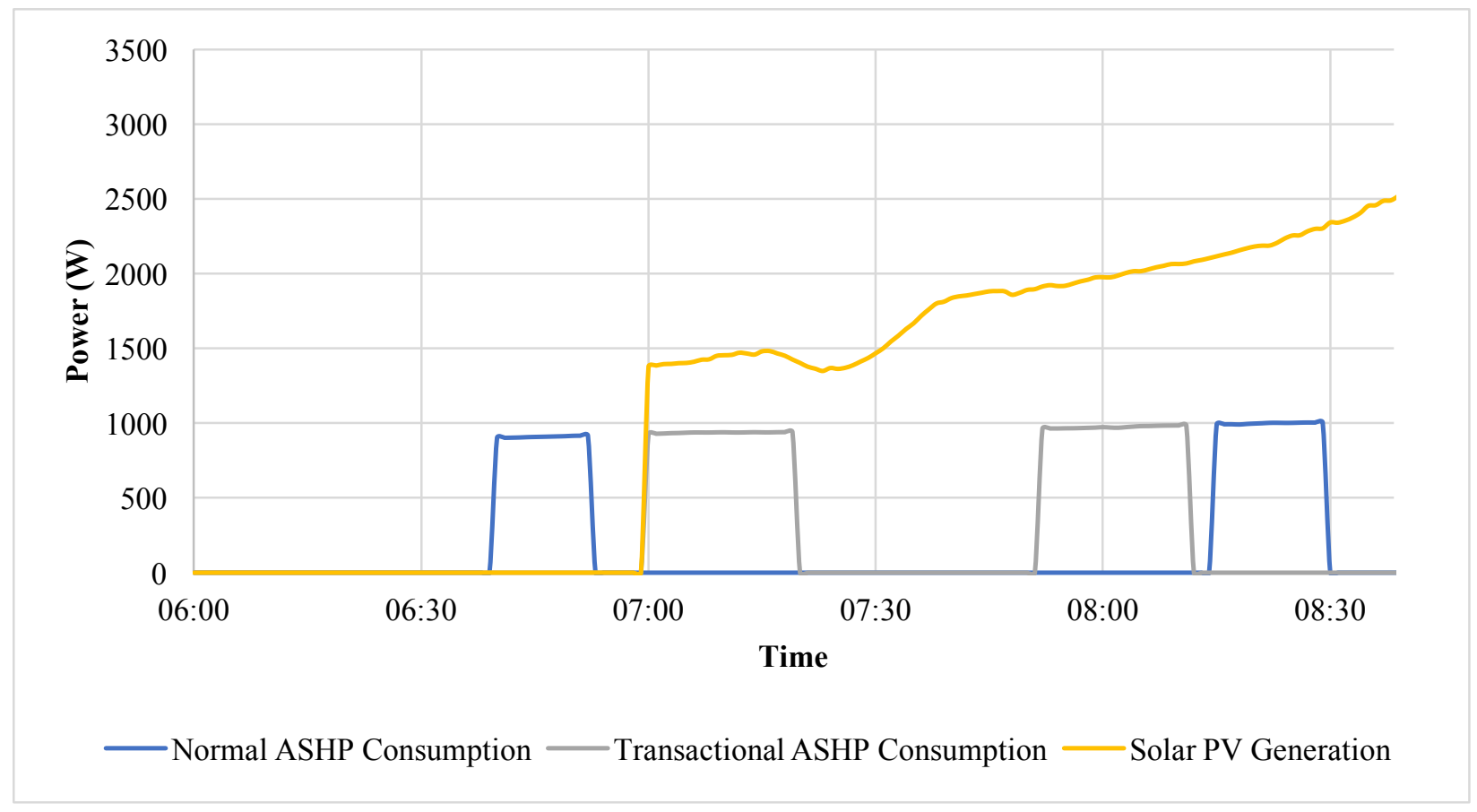

Figure 6-28: An example of the TEMS using flexible loads to align consumption with generation

Table 6-24: Annual on-site renewable energy utilization using for a single house with a $4.08 \mathrm{~kW}$

\begin{tabular}{|c|c|c|c|}
\hline \multicolumn{4}{|c|}{ PV system } \\
\hline System & $\begin{array}{c}\text { On-site Renewable } \\
\text { Utilization (kWh) }\end{array}$ & $\begin{array}{c}\text { Simulated annual } \\
\text { PV generation } \\
(\mathbf{k W h})\end{array}$ & $\begin{array}{c}\text { Percent utilization } \\
(\%)\end{array}$ \\
\hline Normal operation & 821 & \multirow{3}{*}{4,468} & $18.4 \%$ \\
\hline $\begin{array}{l}\text { Transactional } \\
\text { ASHP and PV }\end{array}$ & 987 & & $22.1 \%$ \\
\hline $\begin{array}{l}\text { Transactional } \\
\text { ASHP, PV, and } \\
\text { battery bank }\end{array}$ & 1,645 & & $36.8 \%$ \\
\hline
\end{tabular}

The final conclusion drawn from transactional energy simulations regards the trading price of renewable energy. The TEMS developed in this work functions like a free market economic system, meaning that the negotiated price of electricity is always determined based on the supply and demand of the system. In the Marketplace bid prices may vary between different agents, but once the supply and demand evaluation is complete, all energy is traded at the market clearing price for that moment in time. 
Although a price of $5.0 \notin / \mathrm{kWh}$ for solar energy may seem low, simulations show that solar energy is only traded at this price one third of the time. While the solar PV array in these simulations may be willing to sell its energy for $5.0 \mathrm{k} / \mathrm{kWh}$, the annual average trading price for solar energy has been calculated to be $12.8 \phi / \mathrm{kWh}$.

Table 6-25 summarizes the simulated average annual and seasonal traded price of solar energy. This clearly shows that when solar energy is in excess in the cooling season, when the market experiences increased supply and decreased demand, solar energy trades at a lower cost than in the heating season, when heating energy demand is greater, and solar energy supply is lacking. While it has not been thoroughly investigated, these costs can give insight into the revenue rate that could be possible if actual transactions were made between different participants, perhaps neighbors, in a transactional energy system.

Table 6-25: Traded price of solar energy in a TEMS

\begin{tabular}{|c|c|c|c|}
\hline $\begin{array}{c}\text { Fixed bid price } \\
(\boldsymbol{c} / \mathbf{k} W h)\end{array}$ & $\begin{array}{c}\text { Annual Average } \\
(\boldsymbol{c} / \mathbf{k W h})\end{array}$ & $\begin{array}{l}\text { Heating Season } \\
\quad(\boldsymbol{c} / \mathbf{k W h})\end{array}$ & $\begin{array}{c}\text { Cooling Season } \\
(\mathbf{c} / \mathbf{k W h})\end{array}$ \\
\hline 5.0 & 12.8 & 14.5 & 7.7 \\
\hline 7.5 & 13.9 & 15.2 & 9.7 \\
\hline 10.0 & 14.9 & 15.8 & 11.8 \\
\hline
\end{tabular}

A transactional energy management system can be beneficial for a variety of purposes. It can act as a platform for DR and other forms of utility-consumer interaction, implement control based on thermal comfort preferences, reduce electricity cost and annual consumption by aligning consumption with generation, and act as the basis for inter-household energy trading. These are all achievable using the same system, with no modification to controller design required to produce any of the different results. 


\section{Experimental Case Study Results}

\subsection{Load Shifting Control}

The load shifting experiment was performed intermittently between January - May, 2016. Figure 7-1 shows an example of one day of net load tracking during mid- and on-peak hours. This particular day shows tracking with the initial open-loop controller. On this day, the average daytime outdoor temperature was $-6.1^{\circ} \mathrm{C}$, and $93.5 \%$ of the ASHP load was met by the battery bank during mid and on-peak hours.

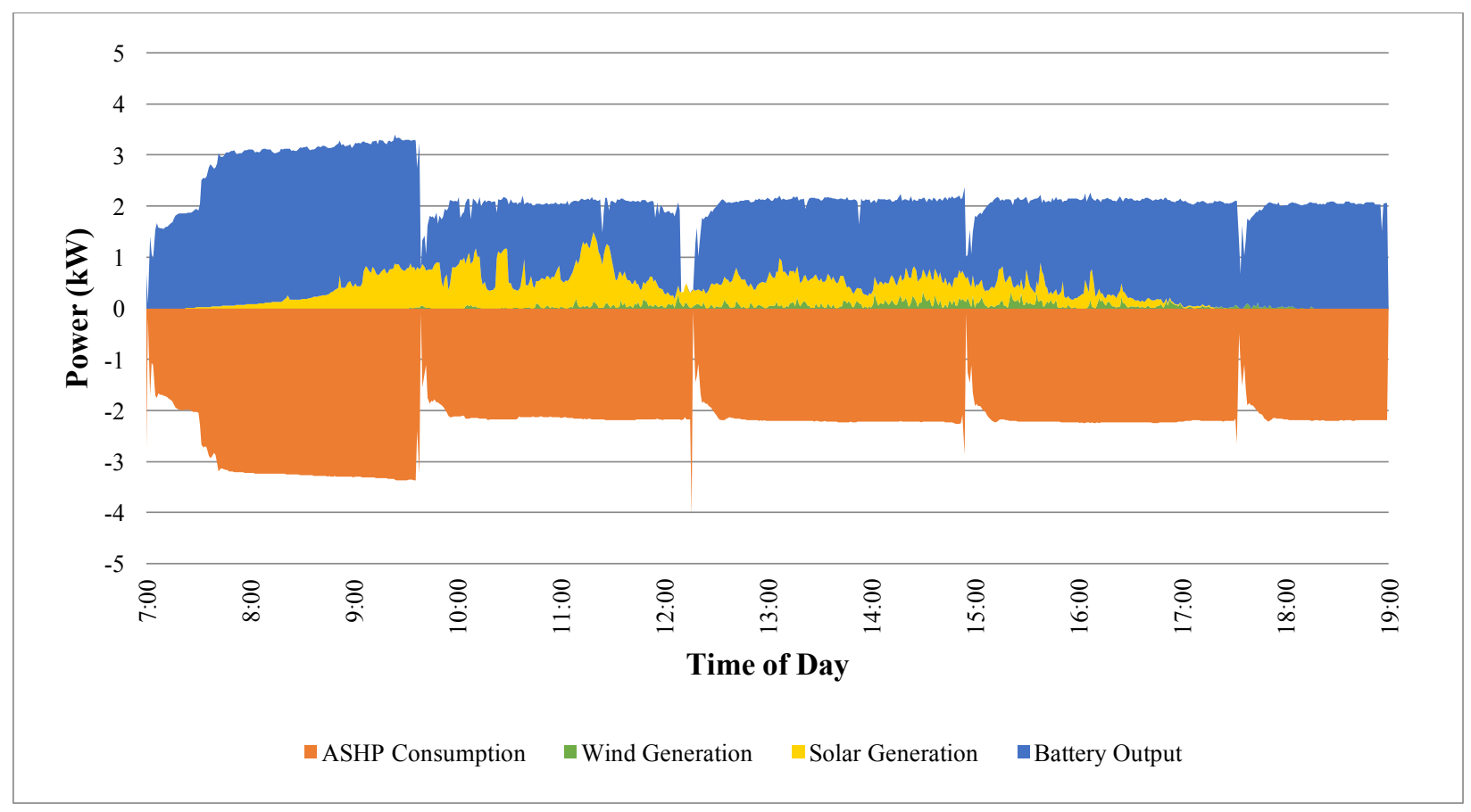

Figure 7-1: Mid-and on-peak ASHP consumption, generation, and battery bank output.

Table 7-1 highlights the accuracy of the controller over each experimental phase. Slightly less accurate tracking accuracy can be observed during set back $\left(18^{\circ} \mathrm{C}\right)$ operation. During the temperature set back, the inverter attempted to match the standby power consumption of the ASHP $(\sim 50 \mathrm{~W})$. It was found that the inverter exhibited a somewhat wavering response at low target power levels. Additionally, a slight steady state error was observed at low power outputs. 
The final controller iteration improved upon steady-state errors and the output at low power levels. The remaining tracking error is associated with slow network speeds, and slow inverter communication/response time.

Table 7-2 shows the outcome of the temperature setback on the power consumption of the ASHP. While these results do show almost a 50\% reduction in energy consumption, they cannot be taken at face-value since outdoor temperatures were warmer during the setback portion of the experiment.

Table 7-1: Percentage of load shifted and days of operation

\begin{tabular}{|l|c|c|}
\hline \multicolumn{1}{|c|}{ Control } & Average percent of load met & Days of operation \\
\hline $\begin{array}{l}\text { Open-loop, normal ASHP } \\
\text { operation }\end{array}$ & $94.3 \%$ & 6 \\
\hline Open-loop, setback operation & $93.3 \%$ & 11 \\
\hline $\begin{array}{l}\text { Closed-loop, both modes of } \\
\text { operation }\end{array}$ & $98.0 \%$ & 3 normal, 3 setback \\
\hline
\end{tabular}

Table 7-2: Effect of temperature setback on power consumption

\begin{tabular}{|l|c|c|}
\hline \multicolumn{1}{|c|}{ Mode of ASHP operation } & $\begin{array}{c}\text { Average ASHP peak-hour } \\
\text { energy consumption (kWh) }\end{array}$ & $\begin{array}{c}\text { Average daytime outdoor } \\
\text { temperatures }\end{array}$ \\
\hline Normal & 19.7 & $-3.1^{\circ} \mathrm{C}$ \\
\hline Setback & 8.9 & $1.1^{\circ} \mathrm{C}$ \\
\hline
\end{tabular}

Having shown that shifting $98 \%$ of an ASHP's peak hour load is possible using hardware that was not designed for this purpose, it is clear that the assumption that the battery bank can effectively match the net load of a building is valid. These results were also obtained using a lead acid battery bank, and it is expected that more rapid current outputs are possible with lithium ion batteries. 


\subsection{Transactional Energy Management System Demonstration}

The integrated LabVIEW-MATLAB communication and control software was used to run the TEMS. Three applications were run separately and simultaneously, representing the Marketplace, HPWH and PV agents. During the demonstration, over 1000 successive bid evaluations were conducted in the marketplace. Figure 7-2 shows a state diagram which highlights how the TEMS functions. While the Marketplace application must be running, agents of any kind can be added or removed from the system at will.

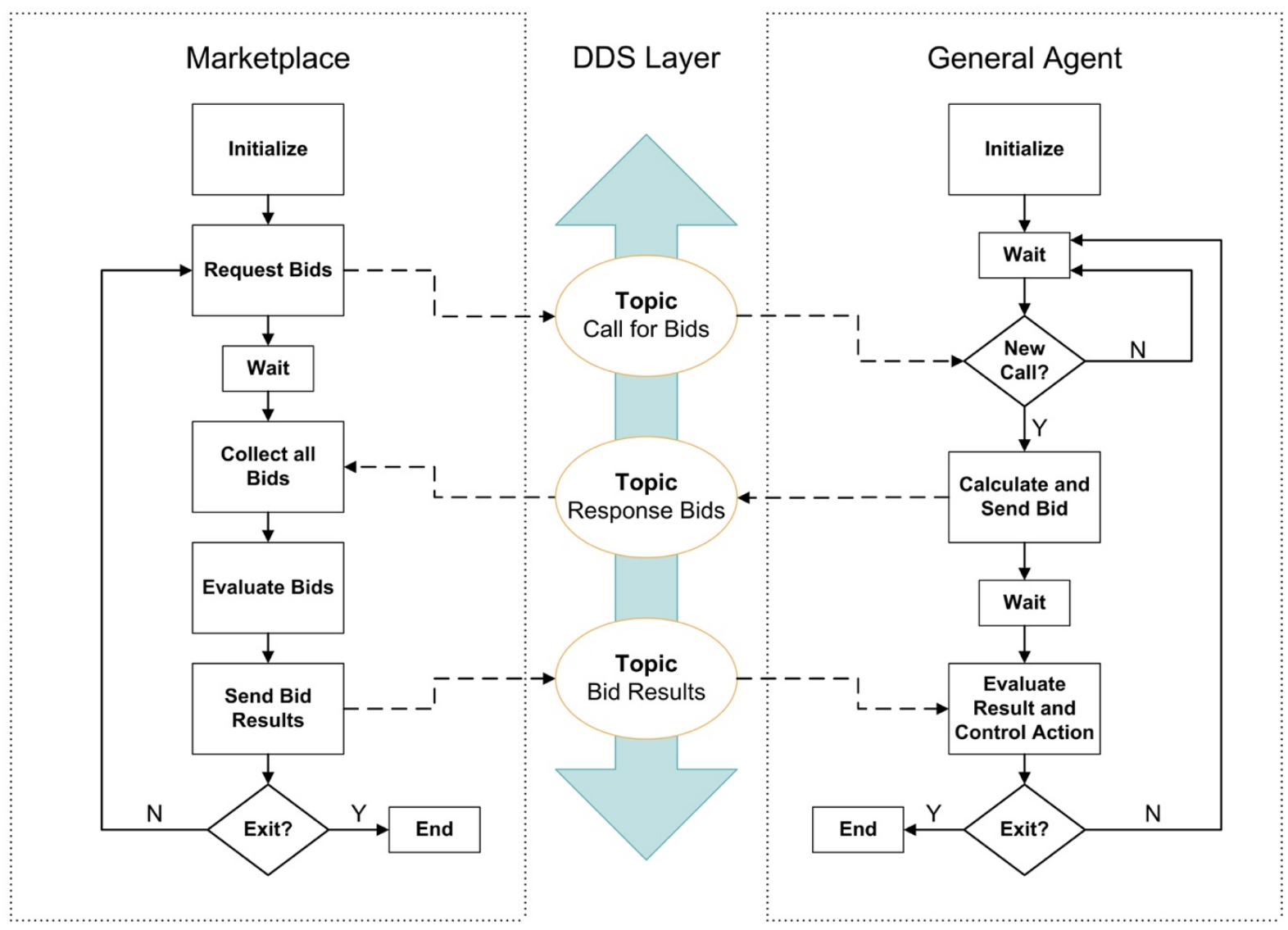

Figure 7-2: Energy Marketplace and Agent state diagrams relative to the DDS layer

The PV and HPWH agents were periodically removed and re-added to the Marketplace throughout the continuous testing period, pictured in Figure 7-3, validating the simple plug-and-play nature of this control system. Currently, the Marketplace acts as the main controller, and is required for 
operation. However, future TEMS implementations could involve fully distributed systems, with direct peer-to-peer communication, negating the need for a main controller.

Figure 7-3 shows the Marketplace's reaction to the removal of the solar PV agent. At the time when the solar PV agent was removed the bid price of the HPWH was less than that of grid energy, so it must be turned off. Since evaluations happened at 10-second intervals in this demonstration, the HPWH is shut off at the end of the first re-evaluation after the PV agent was removed. Eventually, the PV agent was turned back on, and once again began sending supply bids to the Marketplace. After a slight time-delay due to the HPWH's own internal startup controls, the water heater is allowed to power back on.

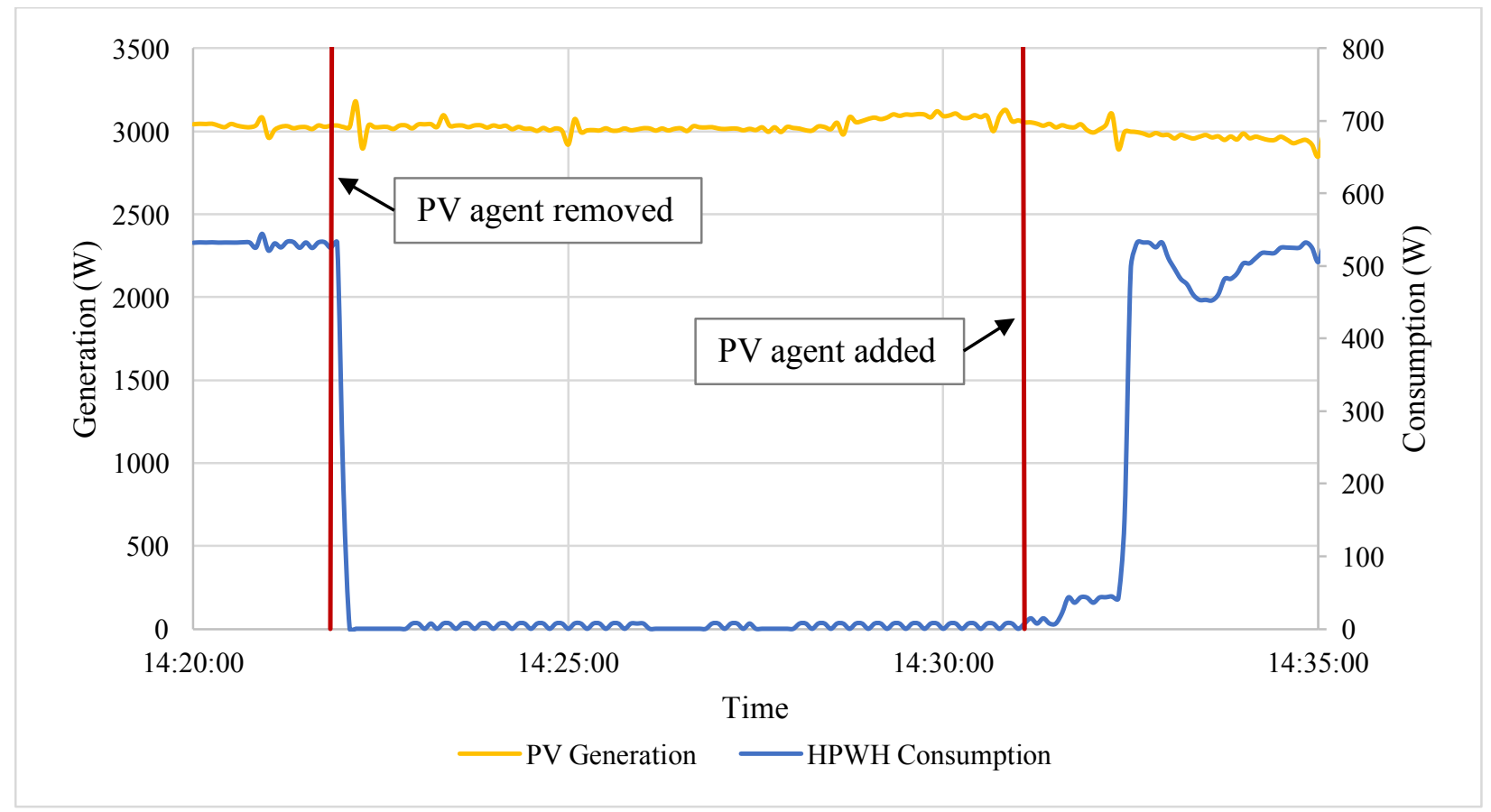

Figure 7-3: Example of the removal of the solar PV agent during the Energy Marketplace demonstration

This demonstration validates the assumption that the communication protocol required for a TEMS is not a barrier for its implementation; open-source software is widely available that may be adapted for this purpose. 


\section{Financial and Emission Analyses}

Financial and emission analyses have been performed on the two deterministic EMSs investigated. These two cases have been selected because they represent conservative scenarios that do not overestimate EMS related savings.

\subsection{Financial Sensitivity to Electricity Prices}

At current battery prices, none of the battery control practices are financially viable; battery bank prices are simply too high. Table 8-1 compares three different battery bank costs, all at $55 \mathrm{kWh}$ in size. The first is a lead-acid battery, while the Enphase and Tesla batteries are both lithium ion. The fact that the lithium ion batteries have double the lifespan of the lead-acid battery alone shows that they are the more cost effective option.

Table 8-1. Battery System Comparison for $55 \mathrm{kWh}$ Battery banks

\begin{tabular}{|l|c|c|c|}
\hline & $\begin{array}{c}\text { Rolls } \\
\text { AGM S2-1275 }\end{array}$ & $\begin{array}{c}\text { Enphase AC } \\
\text { Battery }^{\mathbf{b}}\end{array}$ & $\begin{array}{c}\text { Tesla } \\
\text { Powerwall }^{\mathbf{c}}\end{array}$ \\
\hline Maximum DOD & $50 \%$ & $>95 \%$ & $100 \%$ \\
\hline Unit size & $\sim 2 \mathrm{kWh}$ & $1.2 \mathrm{kWh}$ & $6.4 \mathrm{kWh}$ \\
\hline Units required & 55 & 48 & 9 \\
\hline Cost of system & $\$ 35,750(\mathrm{CAD})$ & $\$ 59,130(\mathrm{CAD})$ & $\$ 32,230(\mathrm{CAD})$ \\
\hline Lifetime & 5 years & 10 years & 10 years \\
\hline
\end{tabular}

$a$ (Rolls Battery Engineering, 2011)

$b$ (Enphase, 2016)

$c$ (Tesla Motors, 2016)

A sensitivity analysis was performed on the cost of electricity, to determine how great the price differential between mid-/on-peak and off-peak would have to be in order to make this process financially viable. This analysis was performed on the medium-consumption house only, and assumed a battery capital cost of $\$ 32,230$, with a lifetime of 10 years.

The analysis involved modifying the mid- and on-peak TOU electricity rates, while keeping the off-peak price of electricity fixed at $0.112 \$ / \mathrm{kWh}$. The TOU rates were modified by a multiplier (i.e. if the on-peak multiplier is 2 , then the on-peak price of electricity is 2 times the current rate). 
Table 8-2 throughTable 8-4 show the annual savings associated with load shifting. Note that if DGs are involved in load shifting, then the baseline case would involve a zero-feedback system. The shaded cells indicate the cases in which the battery bank would payback within its lifetime, based on a simple payback calculation. This analysis shows that the price of mid- and on-peak electricity would have to triple in order for the load shifting practices to become financially viable.

Table 8-2: Annual savings associated with load shifting for a medium house without DGs based on different TOU prices

\begin{tabular}{|c|c|c|c|c|c|}
\hline & \multicolumn{5}{|c|}{ On-peak multiplier } \\
\hline Mid-peak multiplier & $\mathbf{1}$ & $\mathbf{1 . 2 5}$ & $\mathbf{1 . 5}$ & $\mathbf{2}$ & $\mathbf{3}$ \\
\hline $\mathbf{1}$ & $\$ 426$ & $\$ 753$ & $\$ 1,079$ & $\$ 1,731$ & $\$ 3,036$ \\
\hline $\mathbf{1 . 2 5}$ & & $\$ 995$ & $\$ 1,322$ & $\$ 1,974$ & $\$ 3,279$ \\
\hline $\mathbf{1 . 5}$ & & & $\$ 1,565$ & $\$ 2,217$ & $\$ 3,522$ \\
\hline $\mathbf{2}$ & & & & $\$ 2,703$ & $\$ 4,008$ \\
\hline $\mathbf{3}$ & & & & & $\$ 4,980$ \\
\hline
\end{tabular}

Table 8-3: Annual savings associated with basic load shifting for a medium house with a $5 \mathrm{~kW}$ $P V$ system based on different TOU prices

\begin{tabular}{|c|c|c|c|c|c|}
\hline \multicolumn{5}{|c|}{ On-peak multiplier } \\
\hline Mid-peak multiplier & $\mathbf{1}$ & $\mathbf{1 . 2 5}$ & $\mathbf{1 . 5}$ & $\mathbf{2}$ & $\mathbf{3}$ \\
\hline $\mathbf{1}$ & $\$ 289$ & $\$ 512$ & $\$ 735$ & $\$ 1,180$ & $\$ 2,071$ \\
\hline $\mathbf{1 . 2 5}$ & & $\$ 669$ & $\$ 892$ & $\$ 1,338$ & $\$ 2,229$ \\
\hline $\mathbf{1 . 5}$ & & & $\$ 1,050$ & $\$ 1,495$ & $\$ 2,386$ \\
\hline $\mathbf{2}$ & & & & $\$ 1,810$ & $\$ 2,701$ \\
\hline $\mathbf{3}$ & & & & & $\$ 3,332$ \\
\hline
\end{tabular}


Table 8-4: Annual savings associated with load shifting with excess charging for a medium house with a $5 \mathrm{~kW} P V$ system based on different TOU prices

\begin{tabular}{|c|c|c|c|c|c|}
\hline & \multicolumn{5}{|c|}{ On-peak multiplier } \\
\hline Mid-peak multiplier & $\mathbf{1}$ & $\mathbf{1 . 2 5}$ & $\mathbf{1 . 5}$ & $\mathbf{2}$ & $\mathbf{3}$ \\
\hline $\mathbf{1}$ & $\$ 442$ & $\$ 665$ & $\$ 888$ & $\$ 1,333$ & $\$ 2,225$ \\
\hline $\mathbf{1 . 2 5}$ & & $\$ 823$ & $\$ 1,045$ & $\$ 1,491$ & $\$ 2,383$ \\
\hline $\mathbf{1 . 5}$ & & & $\$ 1,203$ & $\$ 1,649$ & $\$ 2,540$ \\
\hline $\mathbf{2}$ & & & & $\$ 1,964$ & $\$ 2,855$ \\
\hline $\mathbf{3}$ & & & & & $\$ 3,486$ \\
\hline
\end{tabular}

While electricity rates tripling may seem drastic, it is clear that prices will increase over time, often beyond the rate of inflation. Figure 8-1 displays historical electricity prices in Ontario. This rate of increase shows that it is not farfetched for the price of on-peak electricity to double over time, as seen between 2008 and 2016.

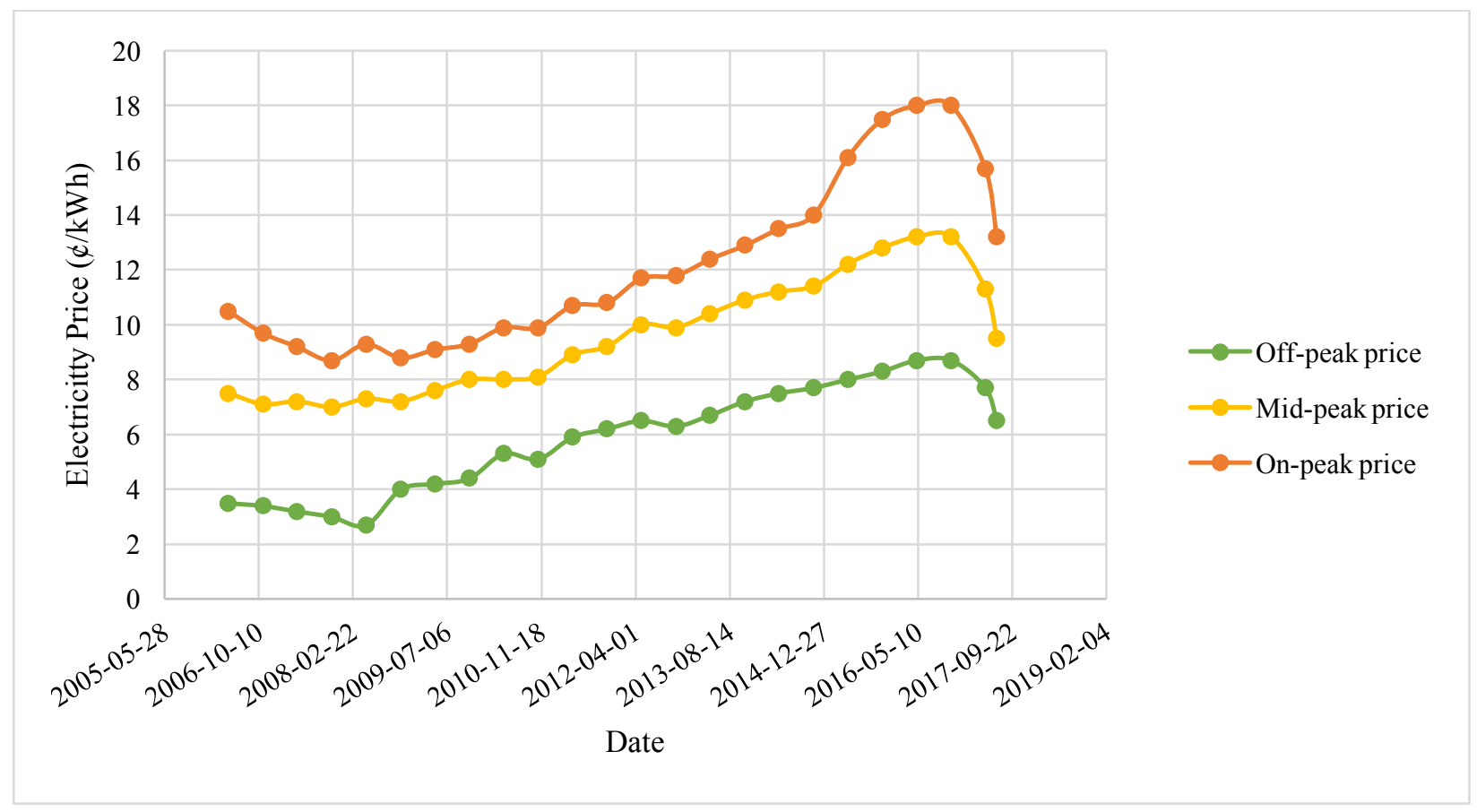

Figure 8-1: Historical Ontario electricity TOU rates, not including marginal costs (OEB, 2017) 
It is not absurd to believe that the capital costs of residential batteries will reduce significantly in the near future. As technological advances are made, the cost of residential batteries will only decrease. If the price of batteries were cut in half, then the price increase of on-peak electricity would only have to double. A price reduction of at half is frankly necessary if this technology is to ever achieve widespread adoption by the public; the price of the battery needs to drop to a reasonable amount, such that at least the average homeowner has the financial means to make the investment.

\subsection{GHG Emission Sensitivity}

A sensitivity analysis with regards to GHG emissions was performed by varying the time of day that charging the battery bank from the grid was allowed. This analysis was only performed on the baseline cases and deterministic EMSs, as these cases are the most likely to be implemented in the real world at this point in time.

Table 8-5 summarizes the annual emissions associated with the various baseline control strategies investigated in this work. Annual emissions were calculated by multiplying electrical load profiles by the Ontario electricity grid's hourly emission factors, described in Section 3.1.2.

Table 8-5: Annual carbon emissions associated with grid electricity for different control strategies

\begin{tabular}{|l|c|c|c|}
\hline & \multicolumn{2}{|c|}{ Average Emissions (kg CO2.eq.year) } \\
\hline \multicolumn{1}{|c|}{ Control Method } & $\begin{array}{c}\text { Low Consumption } \\
\text { Home }\end{array}$ & $\begin{array}{c}\text { Medium } \\
\text { Consumption } \\
\text { Home }\end{array}$ & $\begin{array}{c}\text { High Consumption } \\
\text { Home }\end{array}$ \\
\hline Normal & 1217 & 1417 & 1710 \\
\hline $\begin{array}{l}\text { Zero-feedback with 5 kW } \\
\text { PV }\end{array}$ & 968 & 1147 & 1416 \\
\hline $\begin{array}{l}\text { Normal load shifting } \\
\text { without renewables }\end{array}$ & 1297 & 1485 & 1494 \\
\hline $\begin{array}{l}\text { Basic load shifting with 5 } \\
\text { kW PV }\end{array}$ & 1045 & 1227 & 1418 \\
\hline $\begin{array}{l}\text { Load shifting with excess } \\
\text { charging with 5 kW PV }\end{array}$ & 967 & 1147 & 1763 \\
\hline
\end{tabular}


These results show that both of the basic forms of load shifting (with and without renewables) use grid energy at hours with higher emission factors, compared to when load shifting was not employed. It is not until excess charging is used that emissions associated with load shifting drop below those of the respective baseline cases.

The reason that load shifting has produced greater annual emissions is that the battery is scheduled to charge from the grid at 19:00. It is clear from Figure 3-3 that while electricity may be the cheapest after 19:00, emissions are at their peak. An analysis was performed to determine the optimal time of night to allow recharging from the grid.

Table 8-6 shows the change in associated emissions with regards to the time of night that battery recharging from the grid may begin. The data shown is only for the medium energy consumption home using load shifting without DGs. This analysis shows that the optimal time of night to begin recharging is between 10:00 PM and midnight.

Table 8-6: Emissions associated with load shifting for a medium house without DGs based nighttime charging time

\begin{tabular}{|c|c|c|c|}
\hline $\begin{array}{c}\text { Initial Charging } \\
\text { Time }\end{array}$ & $\begin{array}{l}\text { Normal Emissions } \\
\left(\mathrm{kg} \mathrm{CO}_{2} \text { eq./year) }\right.\end{array}$ & $\begin{array}{c}\text { Load Shifting } \\
\text { Emissions } \\
\left(\mathrm{kg} \mathrm{CO}_{2} \text { eq./year) }\right.\end{array}$ & Percent Change \\
\hline 19:00 & \multirow{9}{*}{1417.1} & 1485.0 & $4.8 \%$ \\
\hline $20: 00$ & & 1433.1 & $1.1 \%$ \\
\hline $21: 00$ & & 1390.0 & $-1.9 \%$ \\
\hline 22:00 & & 1369.5 & $-3.4 \%$ \\
\hline 23:00 & & 1369.9 & $-3.3 \%$ \\
\hline $0: 00$ & & 1373.4 & $-3.1 \%$ \\
\hline 1:00 & & 1387.6 & $-2.1 \%$ \\
\hline $2: 00$ & & 1405.8 & $-0.8 \%$ \\
\hline 3:00 & & 1422.7 & $0.4 \%$ \\
\hline
\end{tabular}

While a 3\% reduction in emissions may seem insignificant, some conclusions can be drawn from this finding. The general public is only just starting to become aware of residential battery technology. While the uptake of batteries in homes has not yet begun, EV sales are increasing significantly. If enough drivers plug in their EVs when they arrive home from work a new peak 
demand will develop. If the supply mix is unchanged, this peak demand will be supplied by the dirtiest energy of the Ontario fuel mix.

Proper energy management will therefore be required with regards to overnight battery charging, for EVs and stand-alone batteries, such that a peak recharging demand is spread out over the entire night time. This could be very effectively managed by a community transactional energy system that ensures that only a certain number of batteries are allowed to recharge from the grid at any given time overnight. 


\section{Conclusions and Recommendations}

This work has investigated the performance of various types of energy management systems for residential buildings with a focus on simplicity and practicality. Various EMSs were examined, and each has shown to have advantages and disadvantages in different cases, which are summarized below. Simulating these EMSs on the same basis allows for the direct comparison between the different control strategies.

\section{Deterministic Energy Management Systems}

The deterministic EMSs investigated in this work use control principles based on fixed functions of time. They are the only candidates for use with lead-acid batteries, ensuring long, steady charging procedures overnight, a necessity for lead-acid battery longevity. These EMSs are also the simplest to design and implement - this work has shown that a load shifting EMS can be easily implemented using "off the shelf" components. While this type of EMS has the least associated savings of all those investigated, this control system is still capable of achieving anywhere from 5 - 19\% annual cost savings depending on load and renewable energy system size.

\section{Optimized Energy Management System}

The optimized EMS investigated in this work was designed as a comparison tool, which was later combined with the MLS and predictive control applications. On its own, the optimized EMS used a custom GA code to determine the best battery management decisions based on two potential states: charging-enabled and discharging-enabled. This optimized EMS showed maximum potential cost savings of $17-25 \%$ annually.

\section{Machine Learning Energy Management System.}

The MLS presents the case of an EMS that learns the historical operation of the system for which it is responsible. It performs GA optimization on historical data to determine what the best course of battery management would have been, and trains a machine learning classification system based on this information. The MLS achieves better cost savings than the deterministic EMS, in the range of $15-22 \%$ annually. The primary downfall of this system is that it requires internal memory to 
store historical data, although with current technological advances this can hardly be seen as a realistic barrier to implementation.

\section{Predictive Control}

Complementary to the MLS, the predictive control investigated in this work uses weather forecasting, black-box modelling (using neural networks), and GA optimization to determine what the best course of action will be. Unfortunately, due to a lack of weather forecast data this control protocol could only be investigated in terms of a 10-day long case study. However, this control protocol did show the best-case of cost savings over the case study period, achieving $42.3 \%$ cost reductions, outcompeting the $40.3 \%$ savings of the MLS over the same period. While this control protocol does show great promise, additional work is required in order to accurately quantify annual potential savings. This EMS not only requires memory to store data, but also depends on an active internet connection for operation.

\section{Transactive Energy Management System}

The final EMS investigated used a transactional-energy framework. The TEMS developed in this work stands apart from the other EMSs in terms of both structure and its potential uses. While the TEMS has not shown the best cost savings of the EMSs investigated, it has shown promise for, multi-building application, DR initiatives, implementing temperature setbacks, and increasing onsite renewable utilization. The experimental work conducted in this thesis has shown that this agent-based control system can be easily implemented using open-source software. The TEMS could be further enhanced by several measures, including the addition of predictive control to influence bidding prices. There is a vast amount of future work that could be conducted to determine additional potential benefits and applications of this type of system.

The EMSs investigated in this work highlight but a small fraction of the possibilities that exist. However, the insight that has been gained can help guide the direction of the implementation of real-world systems. The areas of particular interest for future work involve predictive control and transactional energy. 
While it does provide insight, the predictive control case study is not representative of annual performance. This control strategy should be further expanded, and applied to experimental cases in real-world buildings, under actual weather conditions.

The transactional energy system used in this work covers only one of a vast number of potential control options. This system can be applied to systems of various shapes and sizes, and can be designed in various ways. In the future, it is recommended that this type of system be investigated on both the community level and involving a fully distributed peer-to-peer communication protocol.

Unfortunately, with cost of modern battery banks, these practices are simply not economically viable at the current state. Much of the work conducted in this thesis is reliant upon the assumption that home owners are already eager - and more importantly are capable - of purchasing battery banks for themselves. Until battery bank capital costs decrease, this practice is simply not economically feasible.

Nevertheless, the time to implement sustainable technologies is now. With the widespread implementation of renewable energy and battery energy storage, capital costs of sustainable systems will surely continue to drop. Reducing the initial financial burden on the homeowner is critical in order to allow individuals around the world to make the decision to bring renewable energy, storage, and sustainability into their own homes. 


\section{Appendix}

This work has involved many analyses of large data sets. As such, it would be unrealistic to make all of the data available in text form. Therefore, supplementary data is available through the accompanying electronic submission, or upon request. The following is a list of available data:

- Appliance probability curves

- Hot water draw profile

- Annual consumption load profiles (HVAC, DHW, appliance consumption)

- Annual weather data

- Experimental load shifting data

- MATLAB code

○ House model

- EMS codes 


\section{Bibliography}

Çengel, Y. A., \& Ghajar, A. J. (2010). Heat and mass transfer: Fundamentals \& applications. New York: McGraw-Hill.

A.O.Smith Enterprises Ltd. (2015). Voltex ${ }^{\circledR}$ HYBRID ELECTRIC HEAT PUMP WATER HEATER. Residential Hybrid Electric Heat Pump Water Heaters. Fergus, ON, CAN: A.O.Smith Enterprises Ltd.

ABB. (2014, Sep 12). ABB to enable integration of renewables in Alaskan island microgrid. Retrieved Apr 20, 2016, from ABB: http://www.abb.com/cawp/seitp202/BC90B54CC33D1663C1257D50002FAF58.aspx

ABB. (2016). Microgrid Plus System. Retrieved Apr 21, 2016, from ABB: http://new.abb.com/power-generation/power-plant-automation/microgrid-plus-system

ABB. (2016). Microgrid Solutions. Retrieved Apr 21, 2016, from ABB: http://new.abb.com/power-generation/microgrids-solutions

Alharbi, W., \& Bhattacharya, K. (2013). Demand Response and Energy Storage in MV Islanded Microgrids for High Penetration of Renewables. Electrical Power \& Energy Conference (EPEC) (pp. 1-6). Halifax: IEEE.

Alibabaei, N. (2016). Operational Optimization of Residential HVAC System using Model Predictive Control Strategy Planning. Toronto, ON, Canada: Ryerson University.

Allgöwer, F., Findeisen, R., \& Nagy, Z. K. (2004). Nonlinear Model Predictive Control: From Theory to Application. J. Chin. Inst. Chem. Engrs, 35(3), 299-315.

Al-Saud, K. A. (2009). Measured versus Calculated Roof Peak Sol-air Temperature in Hot-arid Regions. Journal of Architecture and Planning Research, 21(1), 1-7.

Amirirad, A. (2016). Heat Pump Water Heater for Cold Climate - Canada. A thesis presented to Ryerson University. Toronto, ON, Canada: Ryerson University.

Arcos Avilés, D., Guinjoan, F., Barricarte, J., Marroyo, L., Sanchis, P., \& Valderrama, H. (2012). Battery management fuzzy control for a grid-tied microgrid with renewable generation. IECON 2012 - 38th Annual Conference on IEEE Industrial Electronics Society (pp. 56075612). IEEE.

Armstrong, M. M., Swinton, M. C., Ribberink, H., Beausoleil-Morrison, I., \& Millette, J. (2009). Synthetically Derived Profiles for Representing Occupant-Driven Electric Loads in Canadian Housing. Journal of Building Performance Simulation, 2(1), 15-30. 
Bansal, R. C., \& Pandey, J. C. (2005). Load forecasting using artificial intelligence techniques: a literature survey. International Journal of Computer Applications in Technology, 22, 109119.

Barnes, M., Dimeas, A., Engler, A., Fitzer, C., Hatziargyriou, N., Jones, C., . . Vandenbergh, M. (2005). Microgrid Laboratory Facilities. 2005 International Conference on Future Power Systems (pp. 1-6). Amsterdam: IEEE.

Bergey Windpower Co. (2017). Small WInd Turbines for Homes \& Businesses. Retrieved Jun 2017, from Bergey Windpower: http://bergey.com/wind-school/residential-wind-energysystems

Bergman, T. L., Lavine, A. S., Incropera, F. P., \& Dewitt, D. P. (2011). Fundamentals of Heat and Mass Transfer. Hoboken: John Wiley \& Sons, Inc.

Bifaretti, S., Bonaiuto, V., Bruni, G., Cordiner, S., Mulone, V., Paglia, C., \& Spagnolo, F. (2015). Domestic Microgrid Energy Management: Model Predictive Control Strategies Experimental Validation. 2015 IEEE 15th International Conference on Environment and Electrical Engineering (EEEIC) (pp. 2221-2225). Rome: IEEE.

Boehm, R. F. (2011). An approach to decreasing the peak electrical demand in residences. Energy Procedia (pp. 337-342). Bangkok: Elsevier.

Candanedo, J. A., \& Athienitis, A. K. (2011, Jun 21). Predictive control of radiant floor heating and solar-source heat pump operation in a solar house. HVAC\&R Research, 17(3), 235256.

Castillo-Cagigal, M., Gutiérrez, A., Monasterio-Huelin, F., Caamaño-Martín, E., Masa, D., \& Jiménez-Leube, J. (2011). A semi-distributed electric demand-side management system with PV generation for self-consumption enhancement. Energy Conversion and Management, 52, 2659-2666.

CEA. (2013). Canada's Electricity Industry. Retrieved Apr 13, 2016, from Canadian Electricity Association: http://www.electricity.ca/media/Electricity101/Electricity101.pdf

Chassin, D. P., Stoustrup, J., Agathoklis, P., \& Djilali, N. (2015). A new thermostat for real-time price demand response: Cost, comfort and energy impacts of discrete-time control without deadband. Applied Energy, 155, 816-825.

CMHC. (2014). The Impact of Heat Pump Water Heaters on Whole-House Energy Consumption. Ottawa: CMHC. 
Corso, G., Di Silvestre, M., Giuseppe, M., Riva, E., \& Zizzo, G. (2010). Multi-objective long term optimal dispatch of distributed energy resources in micro-grids. UPEC (pp. 1-5). Cardiff: IEEE.

De Bruyn, S. (2017, Jun 20). Personal communication. (A. Brookson, Interviewer)

Defraeye, T., Blocken, B., \& Carmeliet, J. (2010, Jul). Convective heat transfer coefficients for exterior building surfaces: Existing correlations and CFD modelling. Energy Conversion and Management.

Department of Energy. (2017). Energy Cost Calculator for Electric and Gas Water Heaters. Retrieved Jun 06, 2017, from Office of Energy Efficiency \& Renewable Energy: https://energy.gov/eere/femp/energy-cost-calculator-electric-and-gas-water-heaters0\#output

DOE. (2012, Mar). Buildings Energy Data Handbook. Retrieved Apr 2016, from U.S. Department of Energy: http://buildingsdatabook.eren.doe.gov/ChapterIntro1.aspx

DOE. (2015). A Common Definition for Zero Energy Buildings. U.S. Department of Energy.

Duffie, J. A., \& Beckman, W. A. (2006). Solar Engineering of Thermal Processes. Hoboken, New Jersey, USA: John Wiley \& Sons, Inc.

Enphase. (2016, Apr 26). Enphase AC Battery. US.

Enriquez, R., Zarzalejo, L., Jiménez, M., \& Heras, M. (2012). Ground reflectance estimation by means of horizontal and vertical radiation measurements. Solar Energy, 3216-3226.

Erge, T., Becker, R., Kröger-Vodde, A., Laukamp, H., Thoma, M., Werner, R., \& Wittwer, C. (2005). Report on improved power management in low voltage grids by the application of the PoMS system. Deliverable 9.3.

Faruqui, A., \& Sergici, S. (2010). Household response to dynamic pricing of electricity - a survey of the empirical evidence. San Francisco: The Brattle Group.

Fernandes, F., Morais, H., Vale, Z., \& Ramos, C. (2014). Dynamic load management in a smart home to participate in demand response events. Energy and Buildings, 82, 592-606.

Fung, A. S., Dembo, A., \& Zhou, J. (2009). Summary of Detailed Audit and Building Simulation on Archetype Sustainable House, Woodbridge, ON. CANCAM 2009 Conference. Halifax.

Gamez Urias, M., Sanchez, E., \& Ricalde, L. (2015). Electrical Microgrid Optimization via a New Recurrent Neural Network. IEEE Systems Journal, 9(3), 945-953. 
Garcia-Torres, F., Valverde, L., \& Bordons, C. (2016). Optimal Load Sharing of Hydrogen-Based Microgrids with Hybrid Storage Using Model Predictive Control. IEEE TRANSACTIONS ON INDUSTRIAL ELECTRONICS.

Gelazanskas, L., \& Gamage, K. A. (2014). Demand side management in smart grid: A review and proposals for future direction. Sustainable Cities and Society, 22-30.

Gholami, R., Shahabi, M., \& Haghifam, M. R. (2011). An efficient optimal capacitor allocation in DG embedded distribution networks with islanding operation capability of micro-grid using a new genetic based algorithm. Electrical Power and Energy Systems, 335-343.

Giacomoni, A. M., Goldsmith, S. Y., Amin, S., \& Wollenberg, B. F. (2012). Analysis, modeling, and simulation of autonomous microgrids with a high penetration of renewables. 2012 IEEE Power and Energy Society General Meeting (pp. 1-6). San Diego: IEEE.

Gipe, P. (1999). Wind Engineering Basics: A Guide to Small and Micro Wind Systems. White River Junction, VT: Chelsea Green Publishing Company.

Gordon, C., \& Fung, A. (2011). Analysis of Time Dependent Valuation of Emission Factors from the Electricity Sector. Sustainable Growth and Applications in Renewable Energy Sources, 295-312.

Hino, H., Shen, H., Murata, N., Wakao, S., \& Hayashi, Y. (2013). A Versatile Clustering Method for Electricity Consumption Pattern Analysis in Households. IEEE Transactions on Smart Grid, 1048-1057.

Howell, R. H., Coad, W. J., \& Sauer, H. J. (2013). Principles of Heating Ventilating and Air Conditioning. Atlanta: ASHRAE.

Hydro Ottawa Ltd. (2016). peaksaver PLUS. Retrieved Apr 11, 2016, from Hydro Ottawa: https://hydroottawa.com/save-energy/residential/peaksaver-plus

IEA. (2011). Technology Roadmap Smart Grids. Paris: International Energy Agency.

IEA. (2015, June). Energy and CO2 trends in the INDC Scenario. Retrieved Apr 20, 2016, from World Energy Outlook: http://www.worldenergyoutlook.org/inde/

IEA. (2015). How 2 Guide for Smart Grids in Distribution Networks. Paris: International Energy Agency.

IEC. (2012). Grid integration of large-capacity Renewable Energy sources and use of largecapacity Electrical Energy Storage. Geneva: International Electrotechnical Commission. 
IEEE. (2011). IEEE Guide for Design, Operation, and Integration of Distributed Resource Island Systems with Electric Power Systems. New York: IEEE.

IESO. (2015). IESO Demand Response Pilot Program. Toronto: IESO.

IESO. (2016). Supply Overview. Retrieved Sep 30, 2016, from IESO: http://www.ieso.ca/Pages/Power-Data/Supply.aspx

Ionela, P., \& Zio, E. (2014). On the microgrid energy management under a predictive control framework. 2014 IEEE Conference on Control Applications (CCA) (pp. 861-866). Antibes: IEEE.

Katipamula, S., Hatley, D. D., Hammerstrom, D. J., Chassin, D. P., \& Pratt, R. G. (2006). Transactive Controls: Market-Based GridWiseTM Controls for Building Systems. Battelle, PNNL, Oak Ridge.

Keshtkar, A., Arzanpour, S., Keshtkar, F., \& Ahmadi, P. (2015, Oct 01). Smart residential load reduction via fuzzy logic, wireless sensors, and smart grid incentives. Energy and Buildings, 104, 165-180.

Khan, A., Naeem, M., Iqbal, M., Qaisar, S., \& Anpalagan, A. (2016). A compendium of optimization objectives, constraints, tools and algorithms for energy management in microgrids. Renewable and Sustainable Energy Reviews, 58, 1664-1683.

Khederzadeh, M. (2013). Optimal Automation Level in Microgrids. 22nd International Conference on Electricity Distribution (pp. 1-4). Stockholm: IET.

Knight, I., Kreutzer, N., Manning, M., Swinton, M., \& Ribberink, H. (2007). European and Canadian non-HVAC Electric and DHW Load Profiles for Use in Simulating the Performance of Residential Cogeneration Systems. Annex 42 of the International Energy Agency, Energy Conservation in Buildings and Community Systems Programme. IEA.

Kroposki, B., Lasseter, R., Ise, T., Morozumi, S., Papathanassiou, S., \& Hatziargyriou, N. (2008). Making Microgrids Work - A Look at Microgrid Technologies and Testing Projects from Around the World. IEEE Power and Energy Magazine, 40-53.

Lidula, N., \& Rajapakse, A. (2011). Microgrids research: A review of experimental microgrids and test systems. Renewable and Sustainable Energy Reviews, 15(2011), 186-202.

Liu, X., Ivanescu, L., Kang, R., \& Maier, M. (2012). Back to Results Real-time household load priority scheduling algorithm based on prediction of renewable source availability. IEEE Transactions on Consumer Electronics, 58(2), 318-326. 
Liu, Y., Yuen, C., Huang, S., Hassan, N. U., Wang, X., \& Xie, S. (2014). Peak-to-Average Ratio Constrained Demand-Side Management With Consumer's Preference in Residential Smart Grid. IEEE Journal of Selected Topics in Signal Processing, 8(6), 1084-1097.

Ma, J., Qin, J., Salsbury, T., \& Xu, P. (2012, Jan). Demand reduction in building energy systems based on economic model predictive control. Chemical Enegineering Science, 67(1), 92100.

Ma, K., Hu, G., \& Spanos, C. J. (2013). Energy Consumption Scheduling in Smart Grid: A NonCooperative Game Approach. Control Conference (ASCC) (pp. 1-6). Istanbul: IEEE.

Manjili, Y. S., Rajaee, A., Jamshidi, M., \& Kelley, B. T. (2012). Intelligent Decision Making for Energy Management in Microgrids with Air Pollution Reduction Policy. System of Systems Engineering (SoSE) (pp. 13-18). Genoa: IEEE.

Marszal, A. J., Heiselberg, P., Bourrelle, J. S., Musall, E., Voss, K., Sartori, I., \& Napolitano, A. (2011). Zero Energy Building - A review of definitions and calculation methodologies. Energy and Buildings, 971-979.

Marzband, M., Sumper, A., Domínguez-García, J. L., \& Gumara-Ferret, R. (2013). Experimental validation of a real time energy management system for microgrids in islanded mode using a local day-ahead electricity market and MINLP. Energy Conservation and Management, 76, 314-322.

Matallanas, E., Castillo-Cagigal, M., Gutiérrez, A., Monasterio-Huelin, F., Caamaño-Martín, E., Masa, D., \& Jiménez-Leube, J. (2012). Neural network controller for Active Demand-Side Management with PV energy in the residential sector. Applied Energy, 91, 90-97.

Mathew, V., Sitaraman, R. K., \& Shenoy, P. (2014). Reducing Energy Costs in Internet-Scale Distributed Systems Using Load Shifting. 2014 Sixth International Conference on Communication Systems and Networks (COMSNETS) (pp. 1-8). Bangalore: IEEE.

McQuiston, F. C., Parker, J. D., \& Spitler, J. D. (2005). Heating, Ventilating and Air Conditioning: Analysis and Design. Hoboken: John Wiley \& Sons, Inc.

Minchala-Avila, L. I., Garza-Castañón, L. E., Vargas-Martínez, A., \& Zhang, Y. (2015). A review of optimal control techniques applied to the energy management and control of microgrids. The 5th International Conference on Sustainable Energy Information Technology (SEIT 2015). 52, pp. 780-787. Greenwich: Elsevier.

Mitchell, M. (1999). An Introduction to Genetic Algorithms. Cambridge, MA, U.S.: MIT Press. 
Moomaw, W., Burgherr, P., Heath, G., Lenzen, M., Nyboer, J., \& Verbruggen, A. (2011). Annex II Methodology. In IPCC Special Report on Renewable Energy Sources and Climate Change Mitigation. Cambridge: Cambridge University Press.

Morais, H., Kádár, P., Faria, P., Vale, Z. A., \& Khodr, H. M. (2010). Optimal scheduling of a renewable micro-grid in an isolated load area using mixed-integer linear programming. Renewable Energy, 35, 151-156.

Nandar, C. (2013). Robust PI control of smart controllable load for frequency stabilization of microgrid power system. Renewable Energy, 16-23.

NFRC. (2006, Jul). Fenestration Heat Transfer Basics. THERM5.2/WINDOW5.2 NFRC Simulation Manual. National Fenestration Rating Council.

NRCan. (2013). Energy Use Data Handbook. Ottawa: Government of Canada.

NRCan. (2014). Energy Markets Fact Book - 2014 - 2015. Natural Resources Canada.

NRCan. (2017). Energy Efficiency Ratings: Windows. Retrieved Jan 15, 2017, from Natural Resources Canada: http://oee.nrcan.gc.ca/pml-lmp/index.cfm?action=app.searchrecherche\&appliance=WINDOWS

NREL. (2007). Source Energy and Emission Factors for Energy Use in Buildings. Golden: Battelle.

NREL. (2016, Sep). U.S. Solar Photovoltaic System Cost Benchmark: Q1 2016.

OEB. (2017, May). Bill Calculator. Retrieved Jun 2017, from Ontario Energy Board: https://www.oeb.ca/consumer-protection/energy-contracts/bill-calculator

OEB. (2017). Historical electricity rates. Retrieved Jul 2017, from Ontario Energy Board: https://www.oeb.ca/rates-and-your-bill/electricity-rates/historical-electricity-rates

Olivares, D. E., Cañizares, C. A., \& Kazerani, M. (2014, Jul). A Centralized Energy Management System for Isolated Microgrids. IEEE Transactions on Smart Grid, 5(4), 1864-1875.

Onda, H., Yamamoto, S., Takeshit, H., Okamoto, S., \& Yamanaka, N. (2014). Peak Load Shifting and Electricity Charges Reduction Realized by Electric Vehicle Storage Virtualization. 2013 2nd AASRI Conference on Power and Energy Systems. 7, pp. 101-106. AASRI.

Ontario Ministry of Energy. (2013). Ontario's Long-Term Energy Plan. Toronto: Queen's Printer for Ontario. 
Palma-Behnke, R., Benavides, C., Lanas, F., Severino, B., Reyes, L., Llanos, J., \& Sáez, D. (2013).

A Microgrid Energy Management System Based on the Rolling Horizon Strategy. IEEE Transactions on Smart Grid, 4(2), 996-1006.

Panasonic. (2015). Lithium-ion Storage Battery System. Japan: Panasonic Corporation.

Parisio, A., Rikos, E., \& Glielmo, L. (2014). A Model Predictive Control Approach to Microgrid Operation Optimization. IEEE Transactions on Control Systems Technology, 22(5), 18131827.

Parkin, R. E. (2015). A note on the extinction coefficient and absorptivity of glass. Solar Energy, 114, 196-197.

PNNL. (2014). Transaction-Based Building Controls Framework, Volume 1: Reference Guide. Battelle, Pacific Northwest National Laboratory, Oak Ridge.

Poulad, M. E., Fung, A. S., He, L., \& Colpan, C. O. (2016). Modelling residential house electricity demand profile and analysis of peaksaver program using ANN: case study for Toronto, Canada. International Journal of Global Warming, 10.

Powerstream. (2016). Powerstream. Retrieved Apr 09, 2016, from peaksaver PLUS: https://www.powerstream.ca/energy-savings/energy-savings-home/peaksaver-plus.html

Qi, W., Liu, J., \& Christofides, P. D. (2011). A distributed control framework for smart grid development: Energy/water system optimal operation and electric grid integration. Journal of Process Control, 21, 1504-1516.

Qi, W., Liu, J., Chen, X., \& Christofides, P. D. (2011, Jan). Supervisory Predictive Control of Standalone Wind/Solar Energy Generation Systems. IEEE Transactions on Control Systems Technology, 19(1), 199-207.

Radhakrishnan, A., \& Selvan, M. P. (2014). Load Scheduling For Smart Energy Management in Residential Buildings with Renewable Sources. Power Systems Conference (NPSC), 2014 Eighteenth National (pp. 1-6). Guwahati: IEEE.

Rawlings, J. B., \& Mayne, D. Q. (2016). Model Predictive Control: Theory and Design. 2. Madison, WI, US: Nob Hill Publishing.

Reddy, Y., Kumar, Y., Kumar, V., \& Raju, K. (2012). Distributed ANNs in a Layered Architecture for Energy Management and Maintenance Scheduling of Renewable Energy HPS Microgrids. Advances in Power Conversion and Energy Technologies (APCET) (pp. 1-6). Mylavaram: IEEE. 
Rolls Battery Engineering. (2011, Sep). Deep Cycle AGM S2-1275 Specifications. Rolls Battery Engineering.

Sadegheih, A. (2011). Optimal design methodologies under the carbon emission trading program using MIP, GA, SA, and TS. Renewable and Sustainable Energy Reviews, 15, 504-513.

Safa, A. A., Fung, A. S., \& Kumar, R. (2015). Comparative thermal performances of a ground source heat pump and a variable capacity air source heat pump systems for sustainable houses. Applied Thermal Engineering, 279-287.

Saxena, S. (2017). Living City Smart Grid Data Management System. Vaughan: Sustainable Technologies Evaluation Program.

Schweppe, F. C., Tabors, R. D., Kirtley, Jr., J. L., Outhred, H. R., Pickel, F. H., \& Cox, A. J. (1980). Homeostatic Utility Control. IEEE Transactions on Power Apparatus and Systems, 99(3), 1151-1163.

Sherman, M. H. (1986). Estimation of Infiltration from Leakage and Climate Indicators. Energy and Buildings, 10, 81-86.

Siano, P. (2013). Demand response and smart grids - A survey. Renewable and Sustainable Energy Reviews, 461-478.

Snowdon, J., \& Ambrosio, R. (2010). The Olympic Peninsula Project. Smart Grid for Smart Cities. New York: IBM Global Energy \& Utilities Industry.

Southwest Windpower, Inc. (2013). Skystream 3.7. Flagstaff, AZ.

Su, W., Wang, J., \& Roh, J. (2014). Stochastic Energy Scheduling in Microgrids With Intermittent Renewable Energy Resources. IEEE Transactions on Smart Grid, 1867-1883.

SWCC. (2016). SWCC Certified Turbines - Small: Compare Ratings. Retrieved Feb 25, 2017, from Small Wind Certification Council - Independent Certification of Wind Turbines: http://smallwindcertification.org/certified-small-turbines/

Tarroja, B., Zhang, L., Wifvat, V., Shaffer, B., \& Samuelsen, S. (2016). Assessing the stationary energy storage equivalency of vehicle-to-grid charging battery electric vehicles. Energy, 106, 673-690.

Tesla Motors. (2016). Tesla Powerwall. Retrieved May 2016, from Tesla Motors: https://www.teslamotors.com/en_CA/POWERWALL

Toronto Hydro. (2012, Feb). Demand Response DR3. Retrieved Apr 12, 2016, from Toronto Hydro: 
http://www.torontohydro.com/sites/electricsystem/electricityconservation/businessconser vation/pages/demandresponse.aspx

U.S. Department of Energy. (2011, Jan 26). Building America Analysis Spreadsheets. Retrieved Mar 10, 2016, from Office of Energy Efficiency \& Renewable Energy: https://energy.gov/eere/buildings/building-america-analysis-spreadsheets

Unamuno, E., \& Barrena, J. A. (2015). Hybrid ac/dc microgrids—Part I: Review and classification of topologies. Renewable and Sustainable Energy Reviews, 52, 1251-1259.

Valverde, L., Bordons, C., \& Rosa, F. (2012). Power Management using Model Predictive Control in a hydrogen-based microgrid. IECON 2012 - 38th Annual Conference on IEEE Industrial Electronics Society (pp. 5669-5676). Montreal: IEEE.

Xu, B., Li, P.-W., \& Chan, C. L. (2012). Extending the validity of lumped capacitance method for large Biot number in thermal storage application. Solar Energy, 86, 1709-1724.

Zakariazadeh, A., Jadid, S., \& Siano, P. (2014). Smart microgrid energy and reserve scheduling with demand response using stochastic optimization. International Journal of Electrical Power \& Energy Systems, 63, 523-533.

Zhang, J., Fung, A. S., \& Jhingan, S. (2014). Analysis and feasibility study of residential integrated heat and energy recovery ventilator with built-in economizer using an excel spreadsheet program. Energy and Buildings, 430-438.

Zhang, Y., Mao, M., Ding, M., \& Chang, L. (2008). Third International Conference on Electric Utility Deregulation and Restructuring and Power Technologies (pp. 2465-2469). Nanjuing: IEEE. 